\title{
Maternal diet matters
}

Citation for published version (APA):

Rouschop, S. H. M. J. (2022). Maternal diet matters: the molecular impact of dietary fat on the offspring's immunologic, metabolic and pulmonary health. [Doctoral Thesis, Maastricht University]. Gildeprint Drukkerijen. https://doi.org/10.26481/dis.20220202sr

Document status and date:

Published: 01/01/2022

DOI:

10.26481/dis.20220202sr

Document Version:

Publisher's PDF, also known as Version of record

\section{Please check the document version of this publication:}

- A submitted manuscript is the version of the article upon submission and before peer-review. There can be important differences between the submitted version and the official published version of record.

People interested in the research are advised to contact the author for the final version of the publication, or visit the DOI to the publisher's website.

- The final author version and the galley proof are versions of the publication after peer review.

- The final published version features the final layout of the paper including the volume, issue and page numbers.

Link to publication

\footnotetext{
General rights rights.

- You may freely distribute the URL identifying the publication in the public portal. please follow below link for the End User Agreement:

www.umlib.nl/taverne-license

Take down policy

If you believe that this document breaches copyright please contact us at:

repository@maastrichtuniversity.nl

providing details and we will investigate your claim.
}

Copyright and moral rights for the publications made accessible in the public portal are retained by the authors and/or other copyright owners and it is a condition of accessing publications that users recognise and abide by the legal requirements associated with these

- Users may download and print one copy of any publication from the public portal for the purpose of private study or research.

- You may not further distribute the material or use it for any profit-making activity or commercial gain

If the publication is distributed under the terms of Article $25 \mathrm{fa}$ of the Dutch Copyright Act, indicated by the "Taverne" license above, 


\section{Maternal diet matters}

The molecular impact of dietary fat on the offspring's immunologic, metabolic and pulmonary health

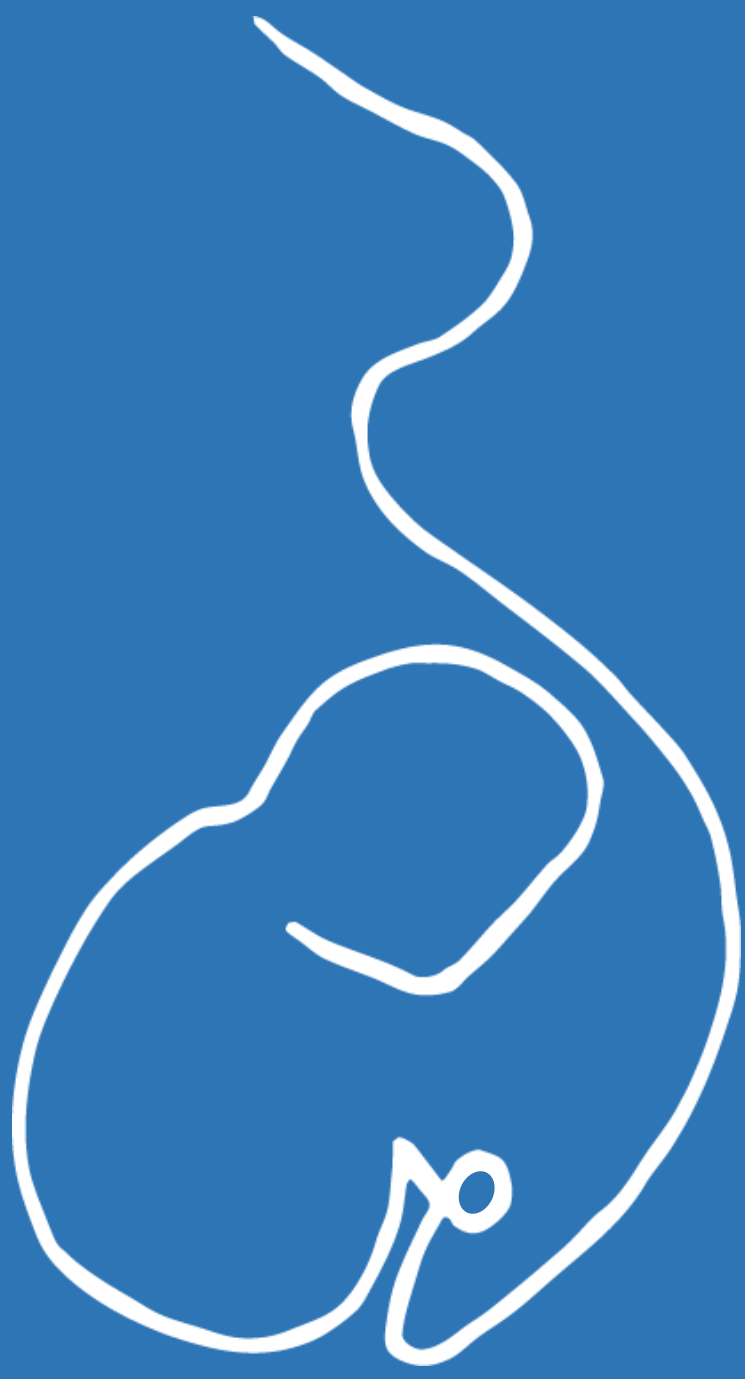




\section{Maternal diet matters}

The molecular impact of dietary fat on the offspring's immunologic, metabolic and pulmonary health

Sven H.M.J. Rouschop 

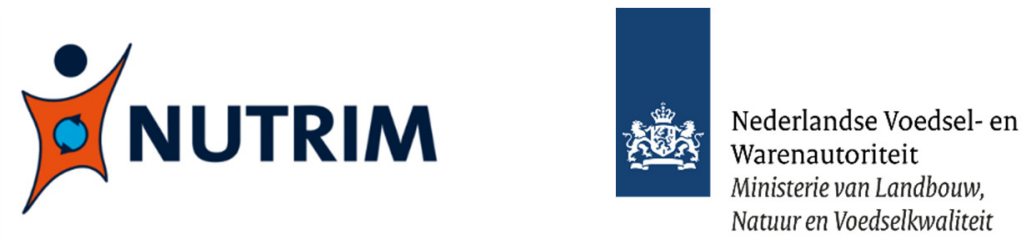

The research described in this thesis was conducted at the department of Pharmacology and Toxicology within NUTRIM School of Nutrition and Translational Research in Metabolism of Maastricht University. The research was funded by the Netherlands Food and Consumer Product Safety Authority (NVWA). Chapter 2 was supported by the Ter Meulen Beurs of the Royal Netherlands Academy of Arts and Sciences (KNAW).

$\begin{array}{ll}\text { Layout } & \text { Sven Rouschop } \\ \text { Cover } & \text { Sven Rouschop \& Gildeprint } \\ \text { Printed by } & \text { Gildeprint } \\ \text { ISBN } & 978-94-6419-386-2\end{array}$

Maternal diet matters: The molecular impact of dietary fat on the offspring's immunologic, metabolic and pulmonary health

(c) Sven H.M.J. Rouschop, 2021, Maastricht, The Netherlands 


\section{Maternal diet matters}

\section{The molecular impact of dietary fat on the offspring's immunologic, metabolic and pulmonary health}

\section{PROEFSCHRIFT}

ter verkrijging van de graad van doctor aan de Universiteit Maastricht op gezag van de Rector Magnificus, Prof. dr. Pamela Habibović volgens het besluit van het College van Decanen, in het openbaar te verdedigen op woensdag 2 februari 2022 om 10:00 uur

door

Sven Henricus Maria Joseph Rouschop

geboren op 23 juni 1992 te Maastricht, Nederland 


\section{Promotores}

Prof. dr. Frederik Jan van Schooten

Prof. dr. Antoon Opperhuizen

\section{Copromotor}

Dr. Roger W.L. Godschalk

\section{Beoordelingscommissie}

Prof. dr. D. Sijm (voorzitter)

Dr. S. Al-Nasiry

Prof. dr. E. Blaak

Prof. dr. P.C. Calder (University of Southampton, United Kingdom)

Prof. dr. T. Nawrot (Universiteit Hasselt, België) 


\section{Contents}

$\begin{array}{lll}\text { Chapter } 1 & \text { General introduction } & 7\end{array}$

Chapter 2 Gene expression and DNA methylation as mechanisms of disturbed metabolism in offspring after exposure to a prenatal high-fat diet

Chapter 3 Perinatal high-fat diet influences ozone-induced responses on pulmonary oxidant status and the molecular control of mitophagy in female rat offspring

Chapter $4 \quad$ Perinatal fish oil diet reduces ex vivo cytokine levels of polarizing $\mathrm{CD}^{+} \mathrm{T}$ cells and induces anemia with microvesicular steatosis in mouse offspring

Chapter $5 \quad$ Maternal fatty acid status during pregnancy versus offspring inflammatory markers: a canonical correlation analysis of the MEFAB cohort

Chapter $6 \quad$ General discussion

Summary

Impact paragraph

Samenvatting

About the author 167

List of publications

171

Dankwoord 


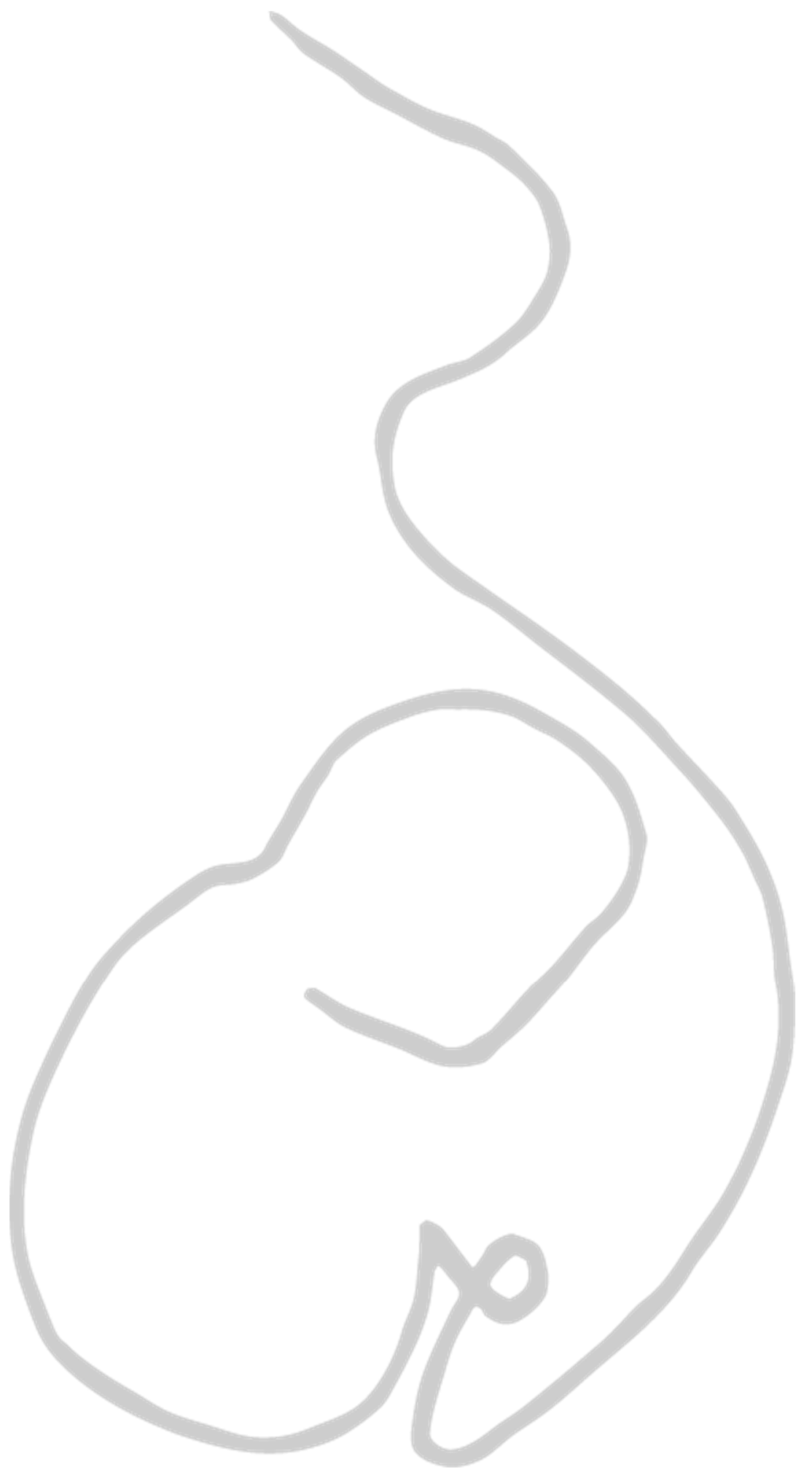


Chapter 1

General introduction 


\section{Fetal programming}

A fetus is highly vulnerable during its development and maternal exposures through lifestyle, diet and environment during pregnancy can greatly determine the health of the offspring at birth and later in life. For instance, smoking during pregnancy increases the risk of adverse outcomes in the child, such as fetal growth restriction, spontaneous abortion, preterm birth, and sudden infant death syndrome [1]. More specifically, offspring health is influenced by maternal diet as well. Alcohol consumption during gestation, for example, is associated with miscarriage, preterm labor, intrauterine growth restriction, and stillbirth [2]. Similarly, consumption of raw meat may result in infection with the parasite Toxoplasma gondii, which can lead to miscarriage, mental retardation, microcephaly, hydrocephalus, and seizures [3]. Besides, offspring health can be affected by a lack of specific nutrients as well. In the early 1990's, it was recognized that a maternal folate deficiency is linked to the incidence of neural tube defects in offspring [4]. To reduce the incidence of these fetal malformations, the Dutch Health Council started recommending women who plan to get pregnant to have a daily intake of $400 \mu \mathrm{g}$ folic acid, starting from four weeks before conception, until eight weeks after conception.

Next to these consequences on the child's health in utero or directly after birth, maternal diet can have long-term effects on offspring health as well, a concept that is known as fetal programming. This idea of fetal programming was clearly shown by the Dutch famine studies, which demonstrated that malnutrition during gestation is associated with detrimental health effects in offspring later in life [5]. During the Dutch famine, which lasted from November 1944 till April 1945, official rations varied from 400 to 1000 calories per day. This caloric restriction was imposed on a previously well-nourished population and was lifted abruptly after the liberation of the Netherlands in May 1945, thereby creating a very specific and narrow window of malnourishment. Observational studies showed that exposure to this famine during gestation was associated with various adverse health effects in the offspring at an adult age, such as increased BMI and adiposity [6,7], decreased glucose tolerance [8,9], an atherogenic lipid profile [10,11], hypertension and increased blood pressure response to stress [12,13], and a higher prevalence, as well as an earlier onset of coronary heart disease $[14,15]$.

\section{Dietary fat}

One specific dietary component that has been associated extensively with offspring health, is dietary fat. Fat is one of the three macronutrients, next to carbohydrates and proteins. In addition to providing energy, fats function as structural building blocks of cell membranes, they carry fat-soluble vitamins, such as vitamin A, D, E and K, and they are precursors of 
hormones and other bioactive molecules. Different types of dietary fat exist, such as phospholipids, sterols and free fatty acids. However, the majority of dietary fats are triglycerides, which are composed of a glycerol backbone attached to three fatty acids (Figure 1). These fatty acids consist of a carboxylic group and a long carbon chain, and can be classified based on their length and saturation. Most of the naturally occurring fatty acids have an even number of carbon atoms and vary in length from four to 28 carbons per chain. Saturation refers to the number of double bonds in the carbon chain. Fatty acids can be saturated (no double bonds), monounsaturated (one double bond), and polyunsaturated (more than one double bond). In fatty acid nomenclature, the length and saturation of a fatty acid are indicated as $x: y n-z$, with $x$ indicating the number of carbon atoms, $y$ indicating the number of double bonds, and $z$ indicating the carbon center at which the first double bond is located, starting counting at the methyl end of the chain. For example, the fatty acid $18: 2 n-6$ has an eighteen-carbon chain, with two double bonds, of which the first is located at the sixth carbon, counted from the methyl end of the chain (Figure 2).

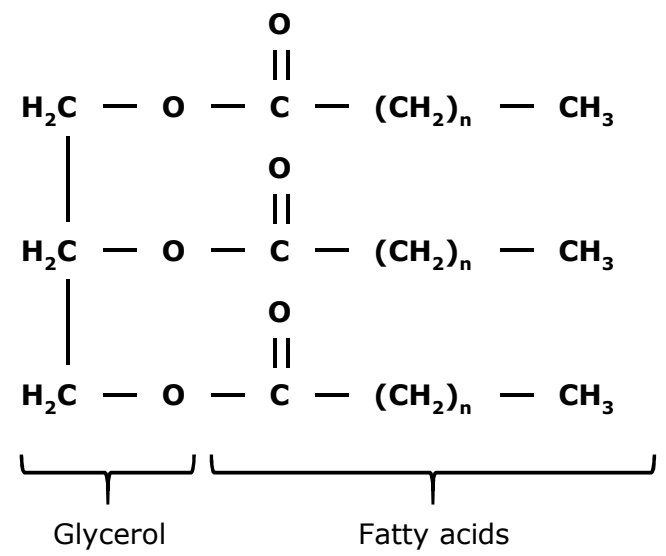

Figure 1. Schematic drawing of a triglyceride.

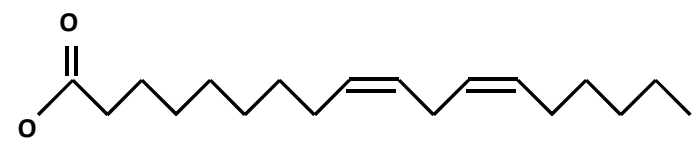

Figure 2. Schematic drawing of the fatty acid 18:2n-6 (linoleic acid). 


\section{Essential fatty acids}

Although many fatty acids can be synthesized de novo in the liver, some fatty acids cannot, meaning that they have to be ingested through food. These so-called essential fatty acids comprise two specific classes of fatty acids: $n-6$ polyunsaturated fatty acids (PUFAs) and n3 PUFAs. The $n-6$ PUFA with the simplest structure is linoleic acid (LA), which is abundant in nuts and seeds of plants like safflower and sunflower. LA can be further elongated and desaturated into more complex derivatives, such as arachidonic acid (AA). In addition, AA is abundant in foods such as meat and eggs. For n-3 PUFAs, the simplest fatty acid is $\alpha$ linolenic acid (ALA), which can be found in seeds of plants like chia and flax. Similar to the conversion of LA into AA, ALA can be further converted into longer-chain derivatives such as eicosapentaenoic acid (EPA) and docosahexaenoic acid (DHA). Furthermore, EPA and DHA are abundant in fatty fish like salmon, herring and menhaden. Both n-6 and n-3 PUFAs have critical functions during fetal growth, including the development of the central nervous system and the retina [16]. Next to this, $n-6$ and $n-3$ PUFAs fulfill significant roles in the regulation of inflammatory responses [17]. These responses, however, differ slightly, with n-6 PUFAs being more pro-inflammatory, whereas n-3 PUFAs are considered to be more anti-inflammatory [17].

\section{Atopy}

Maternal consumption of a diet with a low n-3:n-6 PUFAs ratio has been associated with offspring atopy, corresponding with the pro- and anti-inflammatory properties of $n-6$ and n-3 PUFAs, respectively. Atopy is the predisposition to produce an exaggerated immunoglobulin E (IgE) response to allergens that would otherwise be harmless [18]. Different clinical manifestations of atopy are asthma, allergic rhinitis, atopic dermatitis and food allergy. Although differences exist in these manifestations and their exact pathologies, they share common characteristics such as exacerbations triggered by exposure to an allergen, a Th2-skewed immune response and inflammation [19]. Despite growing knowledge of atopic diseases, their prevalence has increased over the past few decades [20-22]. Concurrent with this growing prevalence of atopic diseases, consumption of proinflammatory n-6 PUFAs has increased, whereas consumption of anti-inflammatory $n-3$ PUFAs has decreased, resulting in the previously formulated hypothesis by Black and Sharpe that the increasing prevalence of atopic diseases may be explained by changes in the $n-6$ PUFA:n-3 PUFA ratio of the diet [23]. Indeed, observational studies demonstrated that maternal intake of n-3 PUFAs such as ALA, EPA and DHA during pregnancy was inversely associated with risk of asthma and asthma-related symptoms in children later in life [2426]. Moreover, human intervention studies showed that maternal supplementation with n- 
3 PUFAs during gestation is able to reduce the risk of asthma, asthma-related symptoms, and having asthma medication prescribed [27-29].

\section{Pulmonary health}

As well as the relationship between asthma in offspring and the specific fatty acid composition of the maternal diet, associations have been found between maternal high-fat diet and pulmonary health in the offspring. For example, both maternal obesity and weight gain during pregnancy are associated with elevated risk of childhood asthma and wheezing $[30,31]$. Similarly, feeding a high-fat diet to female rats and mice during gestation and lactation induces airway hyperresponsiveness and pulmonary inflammation, and increases susceptibility to viral respiratory infection in offspring [32,33]. Furthermore, exposure to a perinatal high-fat diet exacerbates ozone-induced responses on lung function, injury and inflammation in rat offspring [34,35]. It is important to study the underlying mechanisms of these associations and find clues for primary prevention, as the prevalence of inflammatory lung conditions is increasing in the population. According to the World Health Organization (WHO), chronic obstructive pulmonary disease (COPD) and lower respiratory infections were in the top ten global causes of death, as well as in the top ten global causes burden of disease in 2019 [36].

\section{Metabolism}

Next to offspring pulmonary health, maternal high-fat diet has consequences for offspring metabolic health, such as the risk of developing non-alcoholic fatty liver disease (NAFLD). NAFLD is the accumulation of lipids in the liver (hepatic steatosis) in the absence of excess alcohol consumption [37]. NAFLD represents a spectrum of diseases ranging from hepatic steatosis, through steatohepatitis (steatosis in combination with hepatic inflammation), to liver fibrosis and cirrhosis, which ultimately may lead to hepatocellular carcinoma. In normal weight people without any metabolic risk factors, prevalence of NAFLD is about $16 \%$ [38], whereas NAFLD prevalence is $43 \%$ in patients with diabetes [39] and $91 \%$ in obesity patients [40]. Obesity and type 2 diabetes are thus risk factor for developing NAFLD. Various mechanisms contribute to the pathogenic hepatic lipid accumulation in NAFLD (Figure 3) [41]. In type 2 diabetes patients, insulin resistance increases the efflux of free fatty acids from adipose tissue, which are then taken up by the liver. Similarly, excess intake of dietary lipids results in increased hepatic uptake of free fatty acids as well. These hepatic free fatty acids are subsequently converted into triglycerides and stored in the liver, thereby contributing to hepatic steatosis. In addition, insulin resistance leads to increased de novo lipogenesis and decreased $\beta$-oxidation of free fatty acids. Finally, decreased triglyceride export from the liver by very low-density lipoprotein (VLDL) particles contributes to hepatic 
lipid accumulation as well. Several of these characteristics and risk factors of NAFLD have been associated with maternal diet: epidemiological studies have shown that obesity, type 2 diabetes and increased levels of body fat, insulin, and triglycerides occur more often in offspring with obese mothers than offspring with normal-weight mothers [42-45]. Moreover, feeding female mice a high-fat diet during gestation causes offspring to weigh more, have more fat mass and higher leptin levels [46], and induces accumulation of hepatic cholesterol and triglycerides, upregulation of de novo lipid synthesis and steatohepatitis in offspring [47].

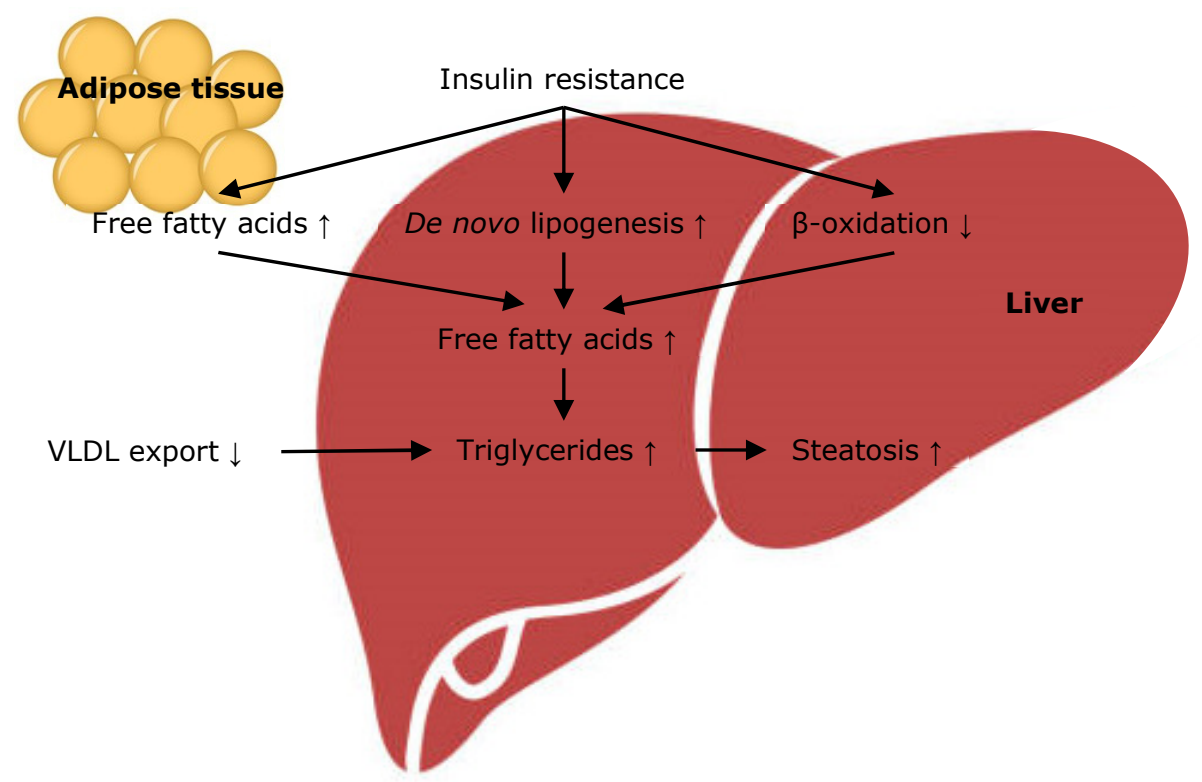

Figure 3. Mechanisms of hepatic lipid accumulation in non-alcoholic fatty liver disease. VLDL, very low-density lipoprotein.

\section{Fetal organ development}

Fetal programming has been proposed to be facilitated by various mechanisms. Firstly, maternal dietary exposures may directly affect the development of a fetal organ, thereby influencing its function during later life. This was suggested by findings from the Dutch famine studies, which showed that the prevalence of obstructive airways disease was increased in adults exposed to famine in mid gestation, but not in adults exposed during early or late gestation [48]. Since bronchial trees grow most rapidly during mid gestation, fetal undernutrition during this developmental period may have affected the structure and physiology of the airways, leading to long-term adverse pulmonary effects [5]. Similarly, the 
Dutch famine study demonstrated that microalbuminuria prevalence was higher in adults exposed to famine during mid gestation, but not early or late gestation [49]. This may be related to nephron numbers increasing rapidly during mid gestation, and thus determining nephron endowment and later-life kidney function [5].

\section{Epigenetics}

In addition to maternal diet directly affecting organ development, prenatal dietary exposures may influence offspring health through epigenetics. Epigenetics are heritable and functionally relevant changes to the genome that do not involve changes in the nucleotide sequence. Various epigenetic mechanisms have been described, such as histone modifications and non-coding RNAs, but the best-characterized epigenetic mechanism is DNA methylation. DNA methylation is the addition of a methyl group at the 5-position of a cytosine nucleotide. This covalent modification primarily occurs at cytosines directly followed by guanosine, a so-called CpG dinucleotide. DNA methylation is established by de novo methyltransferases, whereas demethylation occurs in a multi-step process involving ten-eleven-translocation enzymes [50]. The main way in which DNA methylation affects physiological processes is by regulating gene expression. This regulation of gene expression can be accomplished in several ways. In promoter and enhancer regions, DNA methylation of CpG-rich regions may block binding sites of DNA-binding proteins such as transcription factors [51,52]. Alternatively, methylated CpGs may function as recognition sites for transcriptional repressor protein complexes, thereby inhibiting gene expression [53,54]. Various DNA methylation-related effects have been associated with maternal diet. For instance, feeding mice a high-fat diet during gestation induced both differential DNA methylation and differential gene expression of metabolic and inflammatory genes in offspring $[46,47,55]$. Furthermore, the Dutch famine study showed that individuals who were prenatally exposed to famine had less DNA methylation of the Insulin-like growth factor 2 (IGF2) gene [56]. Given its functions as a fetal growth factor, differential methylation of IGF2 and any related changes in gene expression may thus mediate any adverse effects of prenatal exposure to famine.

\section{Aim and outline of this thesis}

The aim of this thesis was to study the effects of maternal dietary fatty acids and high-fat diet on the molecular responses regarding metabolic, immunologic and pulmonary health. For this purpose, we performed three intervention studies in animals and one observational study in humans. 
In chapter 2, we explored the roles of gene expression and DNA methylation as mechanisms of disturbed metabolism in mouse offspring after exposure to a perinatal high-fat diet. In this study, both parent mice received a high-fat diet or a control diet before and during gestation and throughout lactation, after which offspring were weaned onto a high-fat diet. In offspring, hepatic genome-wide gene expression was assessed, as well as hepatic DNA methylation of selected genes.

The study described in chapter 3 examined whether a perinatal high-fat diet influences ozone-induced responses on pulmonary oxidant status and the molecular control of mitochondrial function and content in rat offspring. For this purpose, female rats were fed a high-fat diet or a control diet before and during gestation, and during lactation. At a young-adult age, offspring were acutely exposed to filtered air or ozone, after which offspring lungs were examined for markers related to oxidative stress and the regulation of mitochondrial function and content.

For chapter 4, we studied the effect of a maternal fish oil diet during gestation and lactation on ex vivo polarization of naïve $\mathrm{CD}^{+} \mathrm{T}$ cells. These $\mathrm{CD} 4^{+} \mathrm{T}$ cells play a major role in the pathology of atopic diseases, since their polarization is Th2-skewed in atopic individuals. In this study, dams received either a fish oil diet or a control diet during gestation and lactation. At the end of lactation, naïve $\mathrm{CD} 4^{+} \mathrm{T}$ cells were isolated from offspring spleen, polarized ex vivo, and examined for secretion and gene expression of Th-specific cytokines and transcription factors.

In chapter 5, we evaluated the multivariate association between maternal fatty acid status during pregnancy and offspring inflammation by analyzing data from the Maastricht Essential Fatty Acid Birth (MEFAB) cohort using canonical correlation analysis.

In Chapter 6, the main outcomes of the studies in this thesis are discussed in a broader perspective. 


\section{References}

1. Shea, A.K.; Steiner, M. Cigarette smoking during pregnancy. Nicotine \& Tobacco Research 2008, 10, 267-278.

2. DeJong, K.; Olyaei, A.; Lo, J.O. Alcohol use in pregnancy. Clinical obstetrics and gynecology 2019, 62, 142.

3. Kravetz, J.D.; Federman, D.G. Toxoplasmosis in pregnancy. The American journal of medicine 2005, 118, 212-216.

4. Geisel, J. Folic acid and neural tube defects in pregnancy: a review. The Journal of perinatal \& neonatal nursing 2003, 17, 268-279.

5. Roseboom, T.J.; Painter, R.C.; van Abeelen, A.F.; Veenendaal, M.V.; de Rooij, S.R. Hungry in the womb: what are the consequences? Lessons from the Dutch famine. Maturitas 2011, 70, 141-145.

6. Ravelli, A.C.; Van Der Meulen, J.H.; Osmond, C.; Barker, D.J.; Bleker, O.P. Obesity at the age of $50 \mathrm{y}$ in men and women exposed to famine prenatally. The American journal of clinical nutrition 1999, 70, 811-816.

7. Stein, A.D.; Kahn, H.S.; Rundle, A.; Zybert, P.A.; van der Pal-de Bruin, K.; Lumey, L. Anthropometric measures in middle age after exposure to famine during gestation: evidence from the Dutch famine. The American journal of clinical nutrition 2007, 85, 869876.

8. de Rooij, S.R.; Painter, R.C.; Roseboom, T.J.; Phillips, D.; Osmond, C.; Barker, D.; Tanck, M.; Michels, R.; Bossuyt, P.; Bleker, O. Glucose tolerance at age 58 and the decline of glucose tolerance in comparison with age 50 in people prenatally exposed to the Dutch famine. Diabetologia 2006, 49, 637-643.

9. Ravelli, A.C.; van der Meulen, J.H.; Michels, R.; Osmond, C.; Barker, D.J.; Hales, C.; Bleker, O.P. Glucose tolerance in adults after prenatal exposure to famine. The Lancet 1998, 351, 173-177.

10. Lumey, L.H.; Stein, A.D.; Kahn, H.S.; Romijn, J. Lipid profiles in middle-aged men and women after famine exposure during gestation: the Dutch Hunger Winter Families Study. The American journal of clinical nutrition 2009, 89, 1737-1743.

11. Roseboom, T.J.; van der Meulen, J.H.; Osmond, C.; Barker, D.J.; Ravelli, A.C.; Bleker, O.P. Plasma lipid profiles in adults after prenatal exposure to the Dutch famine. The American journal of clinical nutrition 2000, 72, 1101-1106.

12. Stein, A.D.; Zybert, P.A.; Van der Pal-de Bruin, K.; Lumey, L. Exposure to famine during gestation, size at birth, and blood pressure at age $59 \mathrm{y}$ : evidence from the Dutch Famine. European journal of epidemiology 2006, 21, 759-765.

13. Painter, R.C.; de Rooij, S.R.; Bossuyt, P.M.; Phillips, D.I.; Osmond, C.; Barker, D.J.; Bleker, O.P.; Roseboom, T.J. Blood pressure response to psychological stressors in adults after prenatal exposure to the Dutch famine. Journal of hypertension 2006, 24, 1771-1778.

14. Roseboom, T.J.; van der Meulen, J.H.; Osmond, C.; Barker, D.J.; Ravelli, A.C.; SchroederTanka, J.M.; van Montfrans, G.A.; Michels, R.P.; Bleker, O.P. Coronary heart disease after prenatal exposure to the Dutch famine, 1944-45. Heart 2000, 84, 595-598. 
15. Painter, R.C.; de Rooij, S.R.; Bossuyt, P.M.; Simmers, T.A.; Osmond, C.; Barker, D.J.; Bleker, O.P.; Roseboom, T.J. Early onset of coronary artery disease after prenatal exposure to the Dutch famine-. The American journal of clinical nutrition 2006, 84, 322-327.

16. Greenberg, J.A.; Bell, S.J.; Van Ausdal, W. Omega-3 fatty acid supplementation during pregnancy. Reviews in obstetrics and Gynecology 2008, 1, 162.

17. Miles, E.A.; Calder, P.C. Can early omega-3 fatty acid exposure reduce risk of childhood allergic disease? Nutrients 2017, 9, 784.

18. Vaillant, A.A.J.; Modi, P.; Jan, A. Atopy. StatPearls [Internet] 2020.

19. Yoo, Y.; Perzanowski, M.S. Allergic sensitization and the environment: latest update. Current allergy and asthma reports 2014, 14, 465.

20. Downs, S.; Marks, G.; Sporik, R.; Belosouva, E.; Car, N.; Peat, J. Continued increase in the prevalence of asthma and atopy. Archives of disease in childhood 2001, 84, 20-23.

21. Hopper, J.L.; Jenkins, M.A.; Carlin, J.B.; Giles, G.G. Increase in the self-reported prevalence of asthma and hay fever in adults over the last generation: a matched parent-offspring study. Australian journal of public health 1995, 19, 120-124.

22. Ninan, T.K.; Russell, G. Respiratory symptoms and atopy in Aberdeen schoolchildren: evidence from two surveys 25 years apart. British medical journal 1992, 304, 873-875.

23. Black, P.; Sharpe, S. Dietary fat and asthma: is there a connection? European Respiratory Journal 1997, 10, 6-12.

24. Lumia, M.; Luukkainen, P.; Tapanainen, H.; Kaila, M.; Erkkola, M.; Uusitalo, L.; Niinistö, S.; Kenward, M.G.; Ilonen, J.; Simell, O. Dietary fatty acid composition during pregnancy and the risk of asthma in the offspring. Pediatr Allergy Immu 2011, 22, 827-835.

25. Miyake, Y.; Sasaki, S.; Tanaka, K.; Ohfuji, S.; Hirota, Y. Maternal fat consumption during pregnancy and risk of wheeze and eczema in Japanese infants aged 16-24 months: the Osaka Maternal and Child Health Study. Thorax 2009, 64, 815-821.

26. Pike, K.C.; Calder, P.C.; Inskip, H.M.; Robinson, S.M.; Roberts, G.C.; Cooper, C.; Godfrey, K.M.; Lucas, J.S. Maternal plasma phosphatidylcholine fatty acids and atopy and wheeze in the offspring at age of 6 years. Clinical and Developmental Immunology 2012, 2012.

27. Bisgaard, H.; Stokholm, J.; Chawes, B.L.; Vissing, N.H.; Bjarnadóttir, E.; Schoos, A.-M.M.; Wolsk, H.M.; Pedersen, T.M.; Vinding, R.K.; Thorsteinsdóttir, S. Fish oil-derived fatty acids in pregnancy and wheeze and asthma in offspring. New England Journal of Medicine 2016, 375, 2530-2539.

28. Hansen, S.; Strøm, M.; Maslova, E.; Dahl, R.; Hoffmann, H.J.; Rytter, D.; Bech, B.H.; Henriksen, T.B.; Granström, C.; Halldorsson, T.I. Fish oil supplementation during pregnancy and allergic respiratory disease in the adult offspring. J Allergy Clin Immun 2017, 139, 104111. e104.

29. Olsen, S.F.; Østerdal, M.L.; Salvig, J.D.; Mortensen, L.M.; Rytter, D.; Secher, N.J.; Henriksen, T.B. Fish oil intake compared with olive oil intake in late pregnancy and asthma in the offspring: 16 y of registry-based follow-up from a randomized controlled trial. The American journal of clinical nutrition 2008, 88, 167-175.

30. Forno, E.; Young, O.M.; Kumar, R.; Simhan, H.; Celedón, J.C. Maternal obesity in pregnancy, gestational weight gain, and risk of childhood asthma. Pediatrics 2014, 134, e535-e546. 
31. Zugna, D.; Galassi, C.; Annesi-Maesano, I.; Baïz, N.; Barros, H.; Basterrechea, M.; Correia, S.; Duijts, L.; Esplugues, A.; Fantini, M.P. Maternal complications in pregnancy and wheezing in early childhood: a pooled analysis of 14 birth cohorts. International journal of epidemiology 2015, 44, 199-208.

32. Griffiths, P.S.; Walton, C.; Samsell, L.; Perez, M.K.; Piedimonte, G. Maternal high-fat hypercaloric diet during pregnancy results in persistent metabolic and respiratory abnormalities in offspring. Pediatric research 2016, 79, 278-286.

33. MacDonald, K.D.; Moran, A.R.; Scherman, A.J.; McEvoy, C.T.; Platteau, A.S. Maternal highfat diet in mice leads to innate airway hyperresponsiveness in the adult offspring. Physiological reports 2017, 5, e13082.

34. Gordon, C.; Phillips, P.; Johnstone, A.; Schmid, J.; Schladweiler, M.; Ledbetter, A.; Snow, S.; Kodavanti, U. Effects of maternal high-fat diet and sedentary lifestyle on susceptibility of adult offspring to ozone exposure in rats. Inhalation toxicology 2017, 29, 239-254.

35. Snow, S.J.; Phillips, P.M.; Ledbetter, A.; Johnstone, A.F.; Schladweiler, M.C.; Gordon, C.J.; Kodavanti, U.P. The influence of maternal and perinatal high-fat diet on ozone-induced pulmonary responses in offspring. Journal of Toxicology and Environmental Health, Part $A$ 2019, 82, 86-98.

36. World Health Organization. Global Health Estimates: Life expectancy and leading causes of death and disability. Available online: https://www.who.int/data/gho/data/themes/mortality-and-global-health-estimates (accessed on 13-08-2021).

37. Than, N.N.; Newsome, P.N. A concise review of non-alcoholic fatty liver disease. Atherosclerosis 2015, 239, 192-202.

38. Amarapurkar, A.; Ghansar, T. Fatty liver: experience from western India. Annals of hepatology 2007, 6, 37-40.

39. Williamson, R.M.; Price, J.F.; Glancy, S.; Perry, E.; Nee, L.D.; Hayes, P.C.; Frier, B.M.; Van Look, L.A.; Johnston, G.I.; Reynolds, R.M. Prevalence of and risk factors for hepatic steatosis and nonalcoholic fatty liver disease in people with type 2 diabetes: the Edinburgh Type 2 Diabetes Study. Diabetes care 2011, 34, 1139-1144.

40. Machado, M.; Marques-Vidal, P.; Cortez-Pinto, H. Hepatic histology in obese patients undergoing bariatric surgery. Journal of hepatology 2006, 45, 600-606.

41. Dowman, J.K.; Tomlinson, J.; Newsome, P. Pathogenesis of non-alcoholic fatty liver disease. QJM: An International Journal of Medicine 2010, 103, 71-83.

42. Eriksson, J.G.; Sandboge, S.; Salonen, M.K.; Kajantie, E.; Osmond, C. Long-term consequences of maternal overweight in pregnancy on offspring later health: findings from the Helsinki Birth Cohort Study. Annals of medicine 2014, 46, 434-438.

43. Gaillard, R.; Steegers, E.A.; Duijts, L.; Felix, J.F.; Hofman, A.; Franco, O.H.; Jaddoe, V.W. Childhood cardiometabolic outcomes of maternal obesity during pregnancy: the Generation R Study. Hypertension 2014, 63, 683-691.

44. Hochner, H.; Friedlander, Y.; Calderon-Margalit, R.; Meiner, V.; Sagy, Y.; Avgil-Tsadok, M.; Burger, A.; Savitsky, B.; Siscovick, D.S.; Manor, O. Associations of maternal prepregnancy body mass index and gestational weight gain with adult offspring cardiometabolic risk factors: the Jerusalem Perinatal Family Follow-up Study. Circulation 2012, 125, 1381-1389. 
45. Yu, Z.; Han, S.; Zhu, J.; Sun, X.; Ji, C.; Guo, X. Pre-pregnancy body mass index in relation to infant birth weight and offspring overweight/obesity: a systematic review and metaanalysis. Plos One 2013, 8, e61627.

46. Keleher, M.R.; Zaidi, R.; Shah, S.; Oakley, M.E.; Pavlatos, C.; El Idrissi, S.; Xing, X.; Li, D.; Wang, T.; Cheverud, J.M. Maternal high-fat diet associated with altered gene expression, DNA methylation, and obesity risk in mouse offspring. Plos One 2018, 13, e0192606.

47. Pruis, M.; Lendvai, A.; Bloks, V.; Zwier, M.; Baller, J.; De Bruin, A.; Groen, A.; Plösch, T. Maternal western diet primes non-alcoholic fatty liver disease in adult mouse offspring. Acta Physiologica 2014, 210, 215-227.

48. Lopuhaä, C.; Roseboom, T.; Osmond, C.; Barker, D.; Ravelli, A.; Bleker, O.; van Der Zee, J.; Van Der Meulen, J. Atopy, lung function, and obstructive airways disease after prenatal exposure to famine. Thorax 2000, 55, 555-561.

49. Painter, R.C.; Roseboom, T.J.; Van Montfrans, G.A.; Bossuyt, P.M.; Krediet, R.T.; Osmond, C.; Barker, D.J.; Bleker, O.P. Microalbuminuria in adults after prenatal exposure to the Dutch famine. Journal of the American Society of Nephrology 2005, 16, 189-194.

50. Tough, D.F.; Tak, P.P.; Tarakhovsky, A.; Prinjha, R.K. Epigenetic drug discovery: breaking through the immune barrier. Nature Reviews Drug Discovery 2016, 15, 835-853.

51. Campanero, M.R.; Armstrong, M.I.; Flemington, E.K. CpG methylation as a mechanism for the regulation of E2F activity. Proceedings of the National Academy of Sciences 2000, 97, 6481-6486.

52. Iguchi-Ariga, S.; Schaffner, W. CpG methylation of the cAMP-responsive enhancer/promoter sequence TGACGTCA abolishes specific factor binding as well as transcriptional activation. Genes \& development 1989, 3, 612-619.

53. Jones, P.L.; Veenstra, G.J.C.; Wade, P.A.; Vermaak, D.; Kass, S.U.; Landsberger, N.; Strouboulis, J.; Wolffe, A.P. Methylated DNA and MeCP2 recruit histone deacetylase to repress transcription. Nature genetics 1998, 19, 187-191.

54. Nan, X.; Ng, H.-H.; Johnson, C.A.; Laherty, C.D.; Turner, B.M.; Eisenman, R.N.; Bird, A. Transcriptional repression by the methyl-CpG-binding protein MeCP2 involves a histone deacetylase complex. Nature 1998, 393, 386-389.

55. Seki, Y.; Suzuki, M.; Guo, X.; Glenn, A.S.; Vuguin, P.M.; Fiallo, A.; Du, Q.; Ko, Y.-A.; Yu, Y.; Susztak, K. In utero exposure to a high-fat diet programs hepatic hypermethylation and gene dysregulation and development of metabolic syndrome in male mice. Endocrinology 2017, $158,2860-2872$.

56. Heijmans, B.T.; Tobi, E.W.; Stein, A.D.; Putter, H.; Blauw, G.J.; Susser, E.S.; Slagboom, P.E.; Lumey, L. Persistent epigenetic differences associated with prenatal exposure to famine in humans. Proceedings of the National Academy of Sciences 2008, 105, 17046-17049. 


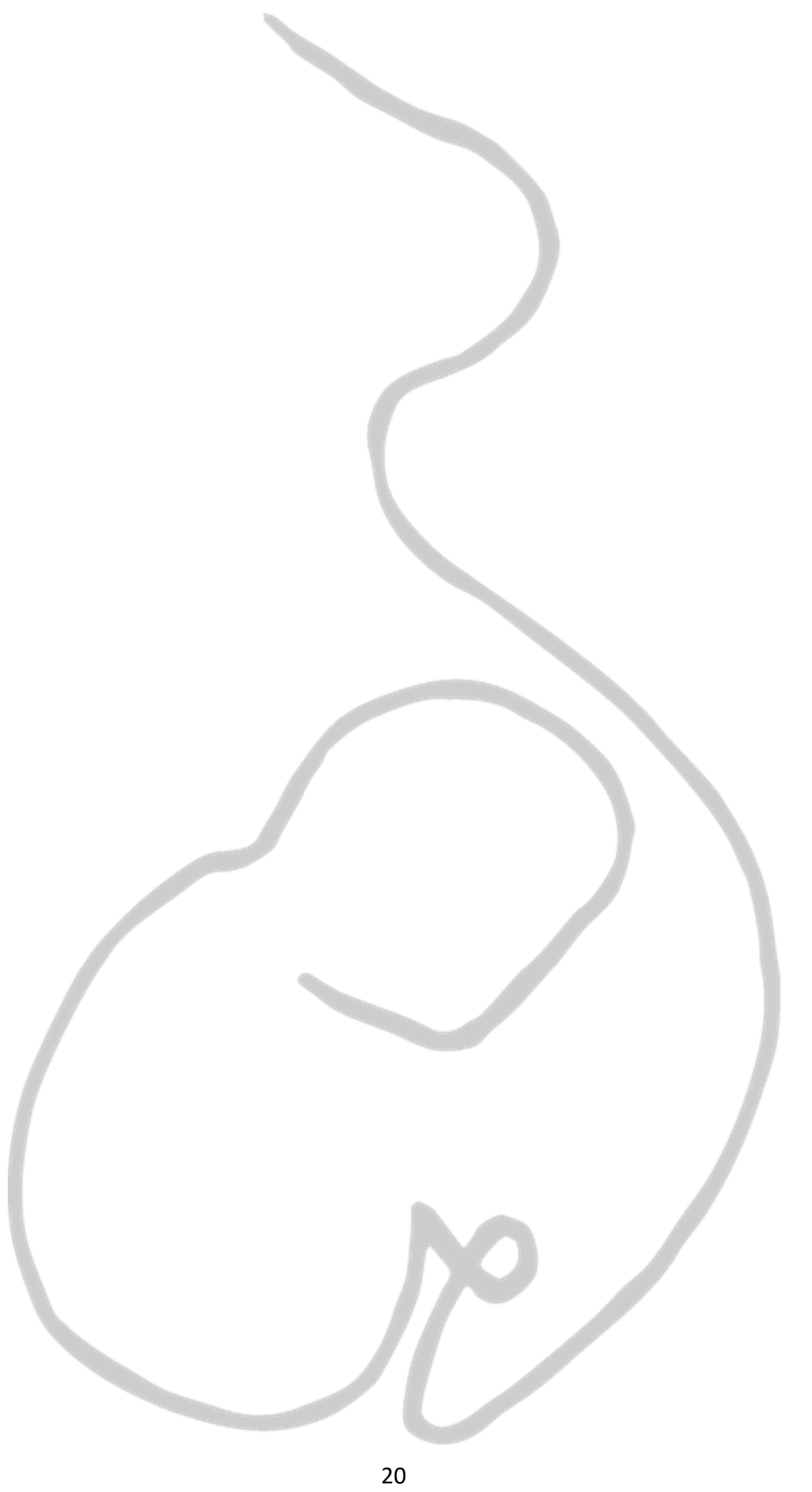




\section{Chapter 2}

\section{Gene expression and DNA methylation as mechanisms of disturbed metabolism in offspring after exposure to a prenatal HF diet}

Sven H. Rouschop, Tanja Karl, Angela Risch, Petronella A. van Ewijk, Vera B. SchrauwenHinderling, Antoon Opperhuizen, Frederik J. van Schooten, Roger W. Godschalk

Journal of lipid research, 2019, 60.7: 1250-1259. 


\begin{abstract}
Exposure to a prenatal high-fat (HF) diet leads to an impaired metabolic phenotype in mouse offspring. The underlying mechanisms, however, are not yet fully understood. Therefore, this study investigated whether the impaired metabolic phenotype may be mediated through altered hepatic DNA methylation and gene expression. We showed that exposure to a prenatal HF diet altered the offspring's hepatic gene expression of pathways involved in lipid synthesis and uptake (SREBP), oxidative stress response (Nrf2) and cell proliferation. The downregulation of the SREBP pathway related to previously reported decreased hepatic lipid uptake and postprandial hypertriglyceridemia in the offspring exposed to the prenatal HF diet. The upregulation of the Nrf2 pathway was associated with increased oxidative stress levels in offspring livers. The prenatal HF diet also induced hypermethylation of transcription factor binding sites upstream of $L p i n 1$, a gene involved in lipid metabolism. Furthermore, DNA methylation of Lpin1 transcription factor binding sites correlated with mRNA expression of Lpin1. These findings suggest that the effect of a prenatal HF diet on the adult offspring's metabolic phenotype are regulated by changes in hepatic gene expression and DNA methylation.
\end{abstract}




\section{Introduction}

The parents' diet before and during pregnancy significantly determines their offspring's risk of developing diseases later in life [1-7]. Observational studies have shown that maternal consumption of a high-fat (HF) diet and obesity during pregnancy and lactation predisposes offspring to developing various metabolic disorders, such as obesity and type 2 diabetes, and cardiovascular diseases [8-11]. In mice, a maternal HF diet leads to increased body weight and fat mass and decreased energy expenditure in offspring [12,13]. Similarly, a paternal HF diet before conception is able to increase the offspring's bodyweight and fat mass, and to impair its lipid and glucose metabolism [14,15]. In addition, both maternal and paternal HF diet have been shown to induce non-alcoholic fatty liver disease in mouse offspring, indicated by increased liver size, excessive accumulation of cholesterol and triglycerides (TGs) and eventually hepatic inflammation [13,14]. Moreover, reports studying the influence of both paternal and maternal diet showed that the combination of these diets has an additive effect on mouse offspring health, leading to more prominent negative effects in offspring compared to only a paternal or maternal HF diet $[14,16]$.

Although the effects of a prenatal HF diet on the offspring phenotype have been studied thoroughly, it is less known through which mechanisms these long lasting effects are transmitted. The responsible molecular mechanisms for this have been suggested to involve epigenetics, since both human and animal studies demonstrated that prenatal exposures alter DNA methylation in the offspring $[12,13,17,18]$. In addition, both maternal and paternal HF diet have been shown to modify gene expression, predominantly affecting pathways related to metabolism and inflammation [12,13,17]. Altering these metabolic and inflammatory pathways contributed to the impaired metabolic phenotype in offspring of HF-fed mice. Altogether, this lead to the hypothesis that a prenatal HF diet changes DNA methylation in offspring, which subsequently alters gene expression, thereby affecting the offspring's phenotype.

To test this hypothesis, we studied the effect of a combined paternal and maternal HF diet on hepatic DNA methylation and gene expression in mouse offspring. In this study, both parent mice received a HF or low-fat (LF) diet before and during pregnancy, and throughout lactation, after which offspring was weaned onto a HF diet. Feeding both parents the same diet and weaning offspring onto a HF diet better approximates the current Western situation, in which the diet is characterized by a HF content, and eating patterns are shared by individuals from the same family [19]. Only male offspring was included for analyses, since sex differences are known to occur in the response to a prenatal HF diet [12,20-23]. Previous work showed that at 12 weeks of age, the offspring displayed postprandial 
hypertriglyceridemia, which related to an impaired clearance of lipids from the blood into the liver after a HF meal [24].

To study the mechanisms underlying the impaired metabolic phenotype after prenatal exposure to a HF diet, hepatic genome-wide gene expression was studied using whole transcript microarrays. In addition, hepatic DNA methylation of selected genes was assessed by bisulphite pyrosequencing. This paper shows that exposure to a prenatal HF diet alters gene expression of pathways involved in hepatic lipid synthesis and uptake, oxidative stress response and cell proliferation. Furthermore, the prenatal HF diet induces hypermethylation of transcription factor (TF) binding sites upstream of Lipin 1 (Lpin1), a differentially expressed gene involved in lipid metabolism.

\section{Materials and Methods}

\section{Study design and workflow}

A schematic overview of the study design and workflow is presented in Figure 1.

\section{Animal model}

Animal experiments were approved by the Institutional Ethics Committee on Animal Welfare of Maastricht University. Mice were housed under standard conditions at $25^{\circ} \mathrm{C}$ with a 12-12 hour light-dark cycle with ad libitum access to water and feed. Specific-pathogenfree (SPF) C57BI/6J (Charles River, Saint-Germain-Nuelles, France) parent mice were randomly assigned to either a HF diet (45\% kcal fat, of which $31 \%$ saturated, $36 \%$ monounsaturated, and $33 \%$ polyunsaturated fatty acids; $20 \% \mathrm{kcal}$ protein; and $35 \% \mathrm{kcal}$ carbohydrate; Research Diets, New Brunswick, USA) or a standard chow LF diet ( $9 \%$ kcal fat; $33 \%$ kcal protein; and 58\% kcal carbohydrate; ssniff Spezialdiäten $\mathrm{GmbH}$, Soest, Germany). The diet started 6 weeks before mating and continued throughout gestation and lactation. Maternal food intake and bodyweight were measured weekly [24]. At weaning, all offspring received a $\mathrm{HF}$ diet, thereby creating the experimental groups $\mathrm{HF} / \mathrm{HF}$ and $\mathrm{LF} / \mathrm{HF}$, with the first two letters indicating the parental diet and the last two letters indicating the offspring diet. Offspring was weaned onto a HF diet, since this experimental setup provides a better representation of the Western diet, which is predominantly characterized by a HF content. Litter size and offspring food intake were similar for both experimental groups [24]. Only male offspring was included for further experiments. Phenotypic outcomes in offspring between 12 and 28 weeks of age were previously reported [24]. To study how these effects of the prenatal HF diet were transmitted, livers were obtained from offspring at 12 weeks 
( $n=7$ per group) and 28 ( $n=9$ per group) weeks of age and used for subsequent measurements in gene expression, oxidative stress and DNA methylation.

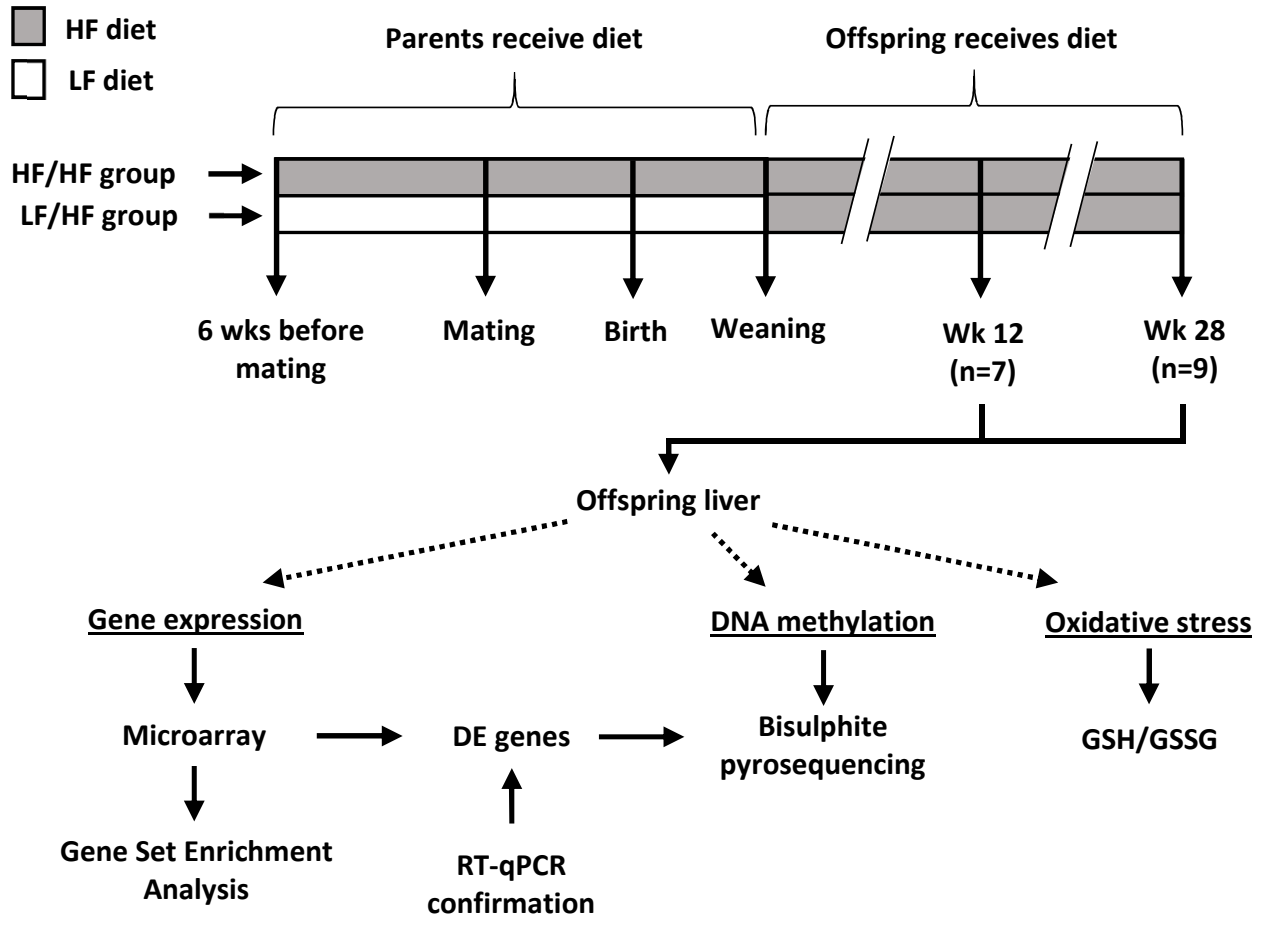

Figure 1. Overview of study design and workflow. Parent mice received a HF or LF diet starting 6 weeks before mating and continuing during pregnancy and lactation. Offspring were weaned onto a HF diet, thereby creating the experimental groups LF/HF and HF/HF, with the first two letters indicating the parental diet and the last two letters indicating the offspring diet. At 12 and 28 weeks of age, offspring livers were collected and analyzed. Gene expression was assessed using microarrays and GSEA. Selected differentially expressed (DE) genes were confirmed with real-time qPCR. DNA methylation of TF binding sites upstream of differentially expressed genes was measured using bisulfite pyrosequencing. Oxidative stress was measured with the GSH/GSSG assay.

\section{Gene expression microarray}

Total RNA from offspring liver was extracted using RNeasy Mini Kit (Qiagen, Venlo, the Netherlands). RNA concentration and purity were assessed using a Nano Drop ND-1000 spectrophotometer (Isogen, IJsselstein, the Netherlands). RNA quality was measured on an Agilent 2100 bioanalyzer (Agilent Technologies, Amsterdam, the Netherlands). All RNA samples had an RNA Integrity Number (RIN) >8.0. RNA (100 ng) was labelled with Whole 
Transcript Sense Target Assay and hybridized to Affymetrix Mouse Gene 1.1 ST arrays (Affymetrix, Santa Clara, USA). Microarrays were analyzed using MADMAX pipeline for statistical analysis of microarray data [25]. Signal intensities were normalized with the robust multichip average (RMA) method and probes were annotated [26]. Microarray data are available through the Gene Expression Omnibus database (GSE123009).

\section{Gene Set Enrichment Analysis}

Functional data analysis of microarray data was performed with Gene Set Enrichment Analysis (GSEA) [27]. GSEA focuses on gene sets (groups of genes that share common biological function or regulation) rather than individual genes. For GSEA, annotated gene sets were used from the databases BioCarta [28], Kyoto Encyclopedia of Genes and Genomes (KEGG) [29], Reactome [30], and WikiPathways [31]. Significantly affected gene sets were selected on a false discovery rate (FDR) q-value of $<0.05$ and were ranked by the normalized enrichment score (NES).

\section{Selection candidate genes for DNA methylation analysis}

Individual genes from the microarray were defined as differentially expressed when comparison of the normalized signal intensities showed a $p$-value $<0.05$ in a two-tailed paired intensity-based moderated t-statistics (IBMT) and an absolute fold-change ( $|F C|)\rangle$ 1.2 [32]. Differentially expressed genes were ranked by $|\mathrm{FC}|$ (HF/HF versus LF/HF at week 12 ) and filtered for involvement in lipid metabolism or inflammation. A selection was made for the top two genes that were differentially expressed at both week 12 and week 28 (Cd163 (Cluster of differentiation 163) and Hmgcr (3-Hydroxy-3-methylglutaryl-CoA reductase)), and the top five genes that were differentially expressed at only week 12 and not at week 28 (Aacs (Acetoacetyl-CoA synthetase), Lpin1 (Lipin 1), Pla2g16 (Phospholipase A2 Group XVI), Saa1 (Serum amyloid A1), and I/1r1 (Interleukin 1 receptor, type I)).

\section{Gene expression RT-qPCR}

To confirm differential expression of candidate genes for DNA methylation, real-time quantitative (q)PCR was used. First, RNA was reverse transcribed into cDNA using iScript (Bio-Rad, Veenendaal, the Netherlands). Real-time quantitative PCR (RT-qPCR) was performed on a Bio-Rad CFX384 Real-Time PCR System using iQ SYBR Green Supermix (BioRad). Primer sequences (Supplemental Table S1) were obtained from PrimerBank [33] and qPrimerDepot [34]. Housekeeping genes Gapdh and Actb were used for normalization. Relative expression levels were quantified with the $\Delta \Delta \mathrm{Ct}$ method [35]. 


\section{Selection transcription factor binding regions}

Candidate genes of which differential expression was confirmed with RT-qPCR were used for subsequent DNA methylation analysis. For this purpose, $\mathrm{CpGs}$ were selected with the potential to regulate expression of the genes Cd163, Hmgcr, Aacs, Lpin1, Saa1, and II1r1. Regions with a high density of TF binding sites were identified $<2000$ bp upstream of the transcription start site (TSS) of each gene (Figure 2A), by using the Gene Transcription Regulation Database (GTRD; version 17.04). GTRD is a database of TF binding sites identified by ChIP-seq experiments [36]. Genomic locations of the selected TF binding regions and $\mathrm{CpGs}$ within those regions are shown in Figure 2B. Methylation of $\mathrm{CpG}$ sites within the identified TF binding regions could potentially influence TF binding and thus regulate gene transcription. Therefore, methylation of these $\mathrm{CpGs}$ was measured using pyrosequencing.

\section{Pyrosequencing}

To assess DNA methylation levels of selected CpG sites, a pyrosequencing approach was used. For this, DNA from offspring liver was extracted using DNeasy Blood \& Tissue Kit (Qiagen) and subjected to bisulphite conversion with EZ DNA Methylation Kit (Zymo Research, Irvine, USA) and subsequently used as template for amplification of the selected TF binding regions. PCR was performed on an Eppendorf Mastercycler nexus (Eppendorf, Nijmegen, the Netherlands), using HotStarTaq DNA polymerase (Qiagen) and dNTP mix (Thermofisher Scientific, Eindhoven, the Netherlands). Biotin-labeled amplicons were captured with Streptavidin Sepharose beads (GE Healthcare, Vienna, Austria) and denatured and annealed to the sequencing primer using the PyroMark Q24 Vacuum Workstation (Qiagen). Amplicons were sequenced on a PyroMark Q24 (Qiagen), using PyroMark Q24 Advanced CpG Reagents (Qiagen). Methylation of each CpG was quantitatively assessed using the PyroMark Q24 Advanced software. Assays and primers for PCR and pyrosequencing (available upon request) were designed with PyroMark Assay Design software.

\section{GSH and GSSG assay}

To assess oxidative stress in offspring livers, reduced and oxidized glutathione (GSH and GSSG, respectively) were measured according to Rahman et al. [37]. In short, liver homogenate was mixed with 5-sulfosalicylic acid to prevent further oxidation of GSH to GSSG. Supernatant of this mixture was used for both GSH and GSSG assessment. GSH was measured by oxidizing it with 5,5-dithiobis-(2-nitro-benzoic acid) (DTNB) to form TNB, which is measurable with a spectrophotometer. For GSSG measurement, samples were first pre- 
incubated with 2-vinylpyridine to deplete all GSH. Subsequently, samples were incubated with NADPH and glutathione reductase, which reduces GSSG into GSH, and simultaneously incubated with DTNB to quantify the amount of GSH through TNB formation. GSH and GSSG concentrations were normalized to the starting amount of liver tissue.

\section{A}

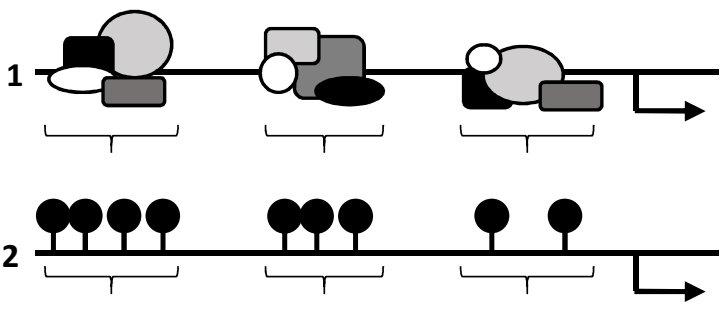

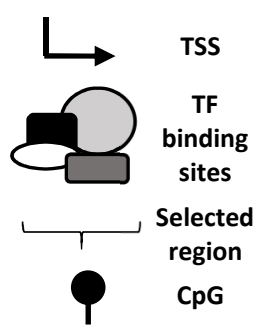

CpG

B
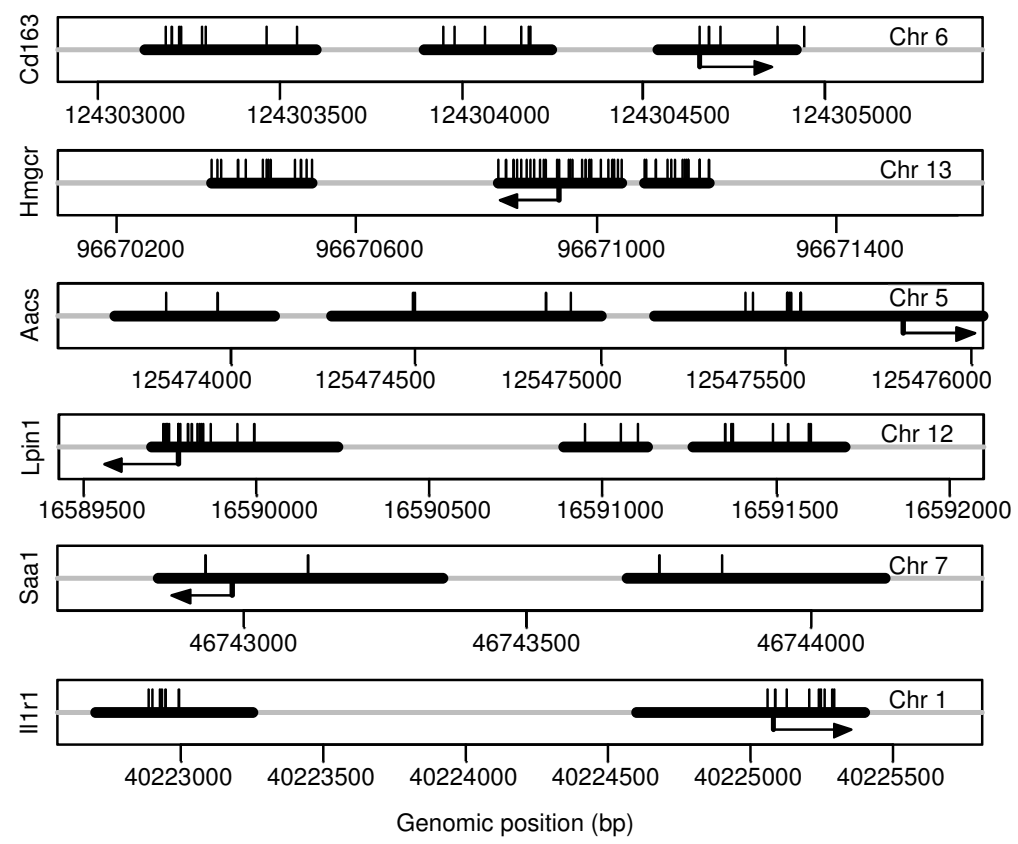

Figure 2. Identification of TF binding regions and selection of $\mathrm{CPG}$ sites for pyrosequencing. $\mathrm{A} 1$ : Regions with a high density of TF binding sites were identified $<2,000 \mathrm{bp}$ upstream of the TSS of a selected gene, based on GTRD. A2: CpG sites within the selected TF binding regions were selected for DNA methylation analysis. B: Genomic positions of TF binding regions (indicated by black horizontal lines) and CpGs within these regions (indicated by vertical lines), which were selected for DNA methylation analysis. Arrows mark TSS. 


\section{Statistical analysis}

For GSH, GSH/GSSG ratio, RT-qPCR, and DNA methylation, differences between groups were tested by two-tailed, independent samples Student $t$ test. For DNA methylation, $p$ values were corrected for multiple testing by computing FDR q-values using the BenjaminiHochberg procedure [38]. For GSSG, differences between groups were tested by two-tailed Mann-Whitney $U$ test. The relation between DNA methylation level of TF binding regions and mRNA expression was described using Pearson correlation coefficient with log transformed mRNA expression levels. $\mathrm{P}<0.05$ or $\mathrm{q}<0.05$ was considered significant. All statistical analyses were performed in R (R Foundation for Statistical Computing, Vienna, Austria).

\section{Results}

\section{A prenatal HF diet alters hepatic expression of gene sets involved in lipid metabolism, oxidative stress response and cell proliferation}

Previous work showed that at 12 weeks of age, HF/HF offspring displayed postprandial hypertriglyceridemia and an impaired clearance of lipids from the blood into the liver after a HF meal [24]. To study whether this impaired metabolic phenotype in HF/HF offspring was related to alterations in hepatic gene expression, a microarray was performed on offspring livers at 12 and 28 weeks of age. As a result, a total of 492 genes was found to be differentially expressed in 12 week old HF/HF offspring, compared to LF/HF offspring. Moreover, GSEA revealed that the prenatal HF diet significantly affected expression of four different pathways in offspring liver at 12 weeks of age, with two being upregulated and two downregulated (Table 1). The two downregulated pathways were related to hepatic lipid biosynthesis and uptake (i.e., Activation of gene expression by SREBP) and cellular movement (i.e., Striated muscle contraction), whereas the upregulated pathways were linked to oxidative stress response [i.e., nuclearfactor (erythroid-derived 2)-like 2 (Nrf2) targets] and intracellular transport (i.e., Kinesins).

In 28 week old offspring, expression of 534 individual genes was altered in HF/HF offspring compared to LF/HF offspring. Furthermore, the prenatal HF diet significantly affected expression of 63 pathways, of which two pathways were upregulated, whereas 61 pathways were downregulated. The two upregulated pathways were both related to Sema4D signaling. Of the downregulated pathways, however, the majority was involved in cell proliferation, having functions in mitosis, cell cycle, proteasomal degradation, Wnt signaling and Hedgehog signaling. In addition, hepatic lipid biosynthesis and uptake was still 
Table 1. Pathways significantly affected by prenatal HF diet in offspring liver at 12 weeks ( $n=7$ per group) and 28 weeks ( $n=9$ per group) of age. For each biological function, the top three (based on normalized enrichment score (NES)) significant pathways are shown. A negative or positive NES indicates that the pathway was downregulated or upregulated, respectively, in HF/HF offspring compared to LF/HF offspring. A complete overview of all significantly affected pathways is listed in Supplemental Table S2. Pathway database: aWikiPathways; ${ }^{\text {b}}$ Reactome; ${ }^{\text {CKEGG; }}{ }^{\text {dBioCarta. }}$

\begin{tabular}{|c|c|c|c|}
\hline Biological Function of Pathway & Pathway & NES & FDR \\
\hline \multicolumn{4}{|l|}{12 weeks } \\
\hline Cellular movement & Striated muscle contraction ${ }^{a}$ & -2.34 & 0.004 \\
\hline Lipid biosynthesis / uptake & Activation of gene expression by SREBPb & -2.32 & 0.002 \\
\hline Oxidative stress response & Nrf2 targets ${ }^{b}$ & 2.60 & $<0.000$ \\
\hline Intracellular transport & Kinesins $^{\mathrm{b}}$ & 2.08 & 0.015 \\
\hline \multicolumn{4}{|l|}{28 weeks } \\
\hline \multirow[t]{3}{*}{ Lipid biosynthesis / uptake } & Cholesterol biosynthesis ${ }^{b}$ & -2.29 & 0.002 \\
\hline & Activation of gene expression by SREBPb & -1.95 & 0.006 \\
\hline & Steroid biosynthesis ${ }^{c}$ & -1.91 & 0.009 \\
\hline \multirow[t]{3}{*}{ Mitochondrial translation } & Mitochondrial translation initiation ${ }^{\mathrm{b}}$ & -2.28 & 0.001 \\
\hline & Mitochondrial translation elongation ${ }^{b}$ & -2.28 & 0.001 \\
\hline & Mitochondrial translation termination ${ }^{b}$ & -2.20 & 0.001 \\
\hline \multirow[t]{3}{*}{ Antigen processing / presentation } & $\begin{array}{l}\text { Cross-presentation of soluble exogenous antigens } \\
\text { (endosomes) }^{\mathrm{b}}\end{array}$ & -2.22 & 0.001 \\
\hline & Antigen processing-Cross presentation ${ }^{\mathrm{b}}$ & -1.77 & 0.028 \\
\hline & ER-Phagosome pathway ${ }^{b}$ & -1.84 & 0.016 \\
\hline \multirow[t]{3}{*}{ Mitosis / Cell cycle } & Autodegradation of $\mathrm{Cdh} 1$ by $\mathrm{Cdh} 1: \mathrm{APC} / \mathrm{C}^{\mathrm{b}}$ & -2.20 & 0.001 \\
\hline & $\begin{array}{l}\text { APC/C:Cdc20 mediated degradation of mitotic } \\
\text { proteins }^{b}\end{array}$ & -2.18 & 0.001 \\
\hline & APC/C:Cdc20 mediated degradation of Securin ${ }^{b}$ & -2.18 & 0.001 \\
\hline \multirow[t]{3}{*}{ Proteasomal degradation } & Proteasome ${ }^{c}$ & -2.15 & 0.001 \\
\hline & Proteasome pathway ${ }^{d}$ & -2.11 & 0.001 \\
\hline & Proteasome degradation ${ }^{a}$ & -1.99 & 0.005 \\
\hline \multirow[t]{2}{*}{ HIV-host interaction } & Vpu mediated degradation of $C D 4^{b}$ & -1.93 & 0.008 \\
\hline & Vif-mediated degradation of $А Р О В Е С 3 G^{b}$ & -1.93 & 0.008 \\
\hline \multirow{3}{*}{$\begin{array}{l}\text { Regulation of transcription and } \\
\text { translation }\end{array}$} & Ribosome biogenesis in eukaryotes ${ }^{c}$ & -2.00 & 0.005 \\
\hline & Eukaryotic transcription initiation ${ }^{a}$ & -1.91 & 0.009 \\
\hline & RNA transport ${ }^{\mathrm{c}}$ & -1.89 & 0.011 \\
\hline \multirow[t]{3}{*}{ Wnt signaling } & Degradation of axin ${ }^{b}$ & -1.89 & 0.011 \\
\hline & Asymmetric localization of PCP proteins ${ }^{b}$ & -1.88 & 0.011 \\
\hline & Degradation of DVL ${ }^{\mathrm{b}}$ & -1.80 & 0.022 \\
\hline \multirow[t]{3}{*}{ Hedgehog signaling } & Hedgehog ligand biogenesis disease ${ }^{b}$ & -1.80 & 0.022 \\
\hline & Degradation of GLI1 by the proteasome ${ }^{b}$ & -1.79 & 0.023 \\
\hline & $\begin{array}{l}\text { Processing defective Hh variants are degraded by the } \\
\text { proteasome }^{b}\end{array}$ & -1.78 & 0.027 \\
\hline \multirow[t]{2}{*}{ Sema4D signaling } & Sema4D in semaphorin signaling ${ }^{b}$ & 2.11 & 0.040 \\
\hline & $\begin{array}{l}\text { Sema4D-induced cell migration and growth cone } \\
\text { collapse }^{b}\end{array}$ & 2.06 & 0.038 \\
\hline
\end{tabular}

downregulated in 28 week old HF/HF offspring, including gene sets Activation of gene expression by SREBP, Cholesterol biosynthesis and Steroid biosynthesis. Altogether, a prenatal HF diet altered hepatic expression of gene sets involved in lipid biosynthesis and uptake, oxidative stress response and cell proliferation. 


\section{A prenatal HF diet increases oxidative stress in offspring liver}

Since the prenatal HF diet upregulated hepatic expression of the Nrf2 pathway in 12 weeks old offspring, we next examined whether livers of these mice also presented increased levels of oxidative stress, which was done by measuring GSH and GSSG. The results show that GSH levels were similar in all four groups, whereas GSSG levels were significantly increased in $\mathrm{HF} / \mathrm{HF}$ offspring compared to LF/HF at 12 weeks of age (Figure 3). Consequently, the GSH/GSSG ratio was significantly decreased in HF/HF offspring compared to $\mathrm{LF} / \mathrm{HF}$ at 12 weeks of age, indicating an increased exposure to oxidative stress in the livers of HF/HF mice. In addition, there was a tendency $(p=0.06)$ for decreased GSH/GSSG ratio in 28 week old compared to 12 week old LF/HF offspring, indicating that oxidative stress tended to increase over time in the control group.

\section{A prenatal HF diet induces hypermethylation of TF binding sites upstream of Lpin1}

To examine whether the observed effect of a prenatal HF diet on hepatic gene expression is regulated by alterations in DNA methylation, further analysis was performed on candidate genes Cd163, Hmgcr, Aacs, Lpin1, Pla2g16, Saa1 and II1r1. These genes were selected based on their involvement in lipid metabolism (Hmgcr, Aacs, Lpin1, and Pla2g16) or inflammation ( $C d 163$, Saa1, and IIr1) and their significant differential expression in the microarray. This differential expression was verified by RT-qPCR, which showed that the prenatal HF diet
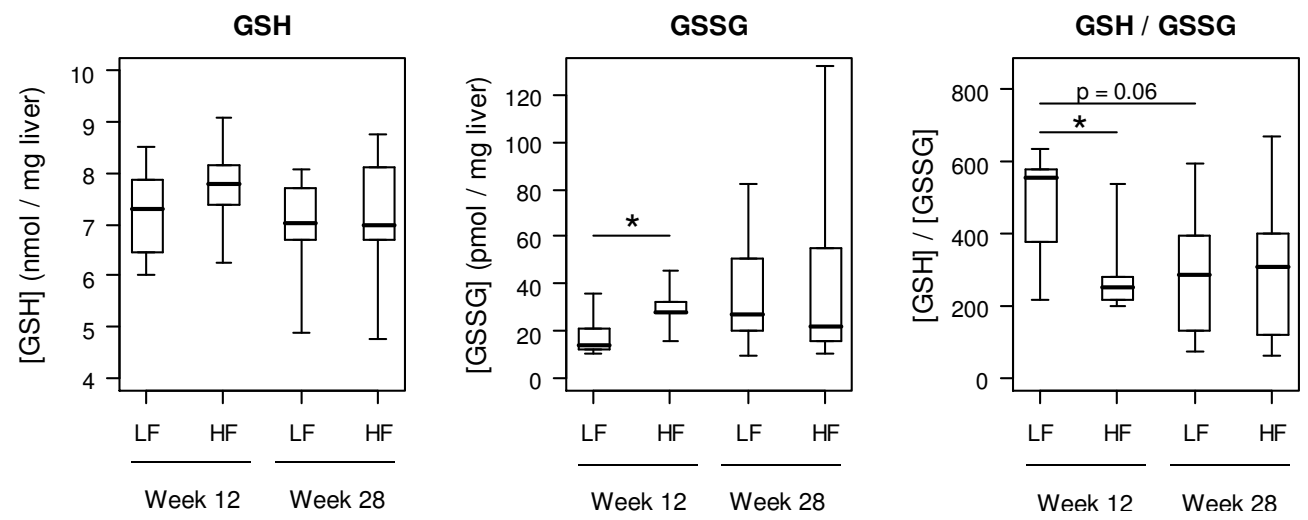

Figure 3. GSH and GSSG concentrations and the ratio between GSH and GSSG concentrations in 12week-old ( $n=7$ for LF and $n=6$ for HF) and 28-week-old ( $n=9$ per group) offspring livers after both parent mice received either a prenatal LF or HF diet. Boxplots represents minimum, first quartile, median, second quartile, and maximum. ${ }^{*} \mathrm{P}<0.05$, based on two-tailed independent samples t-test (GSH and GSH/GSSG) or two-tailed Mann-Whitney $U$ test (GSSG). 
significantly altered hepatic mRNA expression of Cd163 and Hmgcr at 12 and 28 weeks of age, whereas expression of Aacs, Lpin1, Saa1 and IIr1 was only significantly changed at week 12 (Figure 4). For Pla2g16, differential expression could not be confirmed with RTqPCR and it was therefore not included for further analyses.

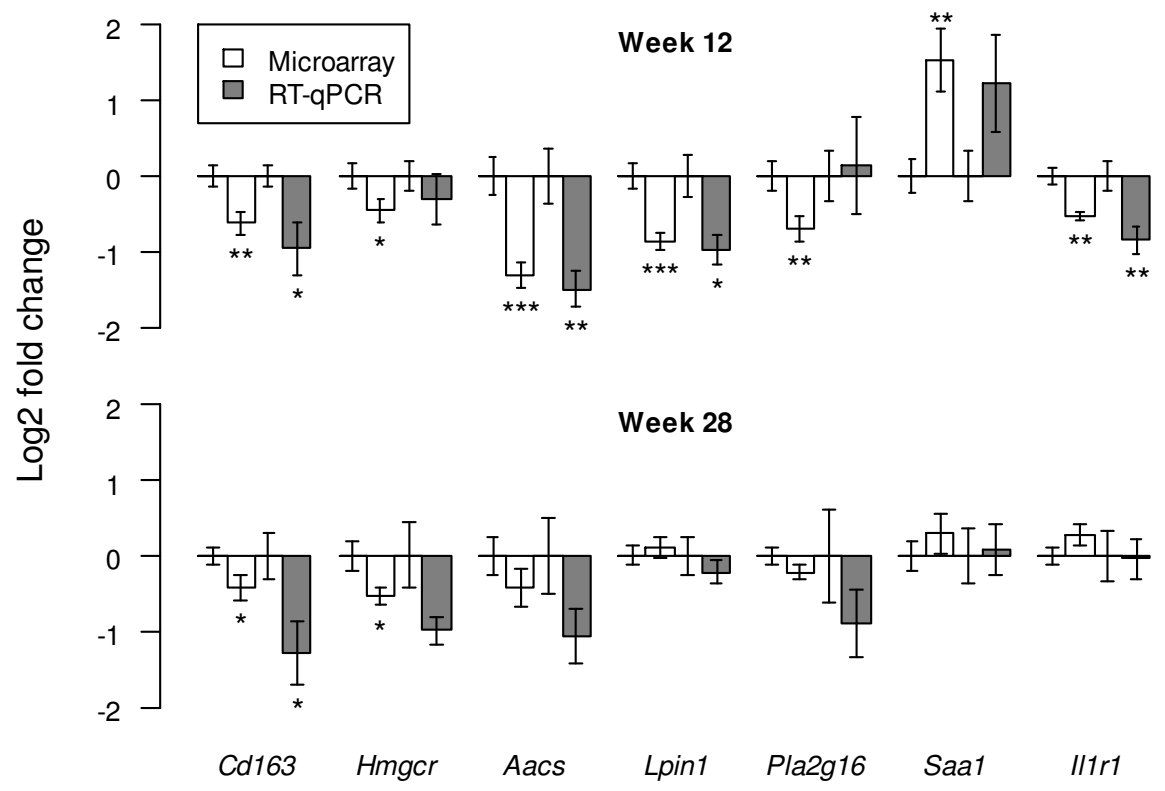

Figure 4. Effect of prenatal HF diet on hepatic mRNA expression of Cd163, Hmgcr, Aacs, Lpin1, Pla2g16, Saa1, and II1r1 in 12-week-old ( $n=7$ per group) and 28-week-old-offspring ( $n=9$ per group) analyzed by microarray and real-time qPCR. Expression was normalized against $A c t b$ and Gapdh and presented relative to control (LF/HF) expression (controls have Log2-fold change of 0 ). Bars indicate mean \pm SEM. ${ }^{*} \mathrm{P}<0.05 ;{ }^{* * P}<0.01 ;{ }^{* * *} \mathrm{P}<0.001$, based on two-tailed paired intensity-based moderated t-statistics (microarray) or two-tailed independent samples t-test (real-time qPCR).

For each of the confirmed genes, methylation was measured at $\mathrm{CpGs}$ within TF binding sites, thereby having the potential to regulate expression of that gene. Analysis showed that at 12 weeks of age, hepatic DNA methylation of Lpin1 TF binding region 2 (Chr12: 16,590,890 - 16,591,130 bp) was significantly increased $(\Delta=7.1 \%, q=0.002)$ in $\mathrm{HF} / \mathrm{HF}$ offspring compared to LF/HF offspring (Figure 5). At 28 weeks of age, there was no longer a significant difference, with Lpin1 region 2 DNA methylation levels of HF/HF offspring partly being restored to the level of LF/HF offspring. No significant changes were observed for Lpin1 TF binding regions 1 and 3, nor for any TF binding region upstream of Cd163, Hmgcr, Aacs, Saa 1 and $/ / 1 r 1$ at 12 or 28 weeks of age (Supplemental Figure S1). 


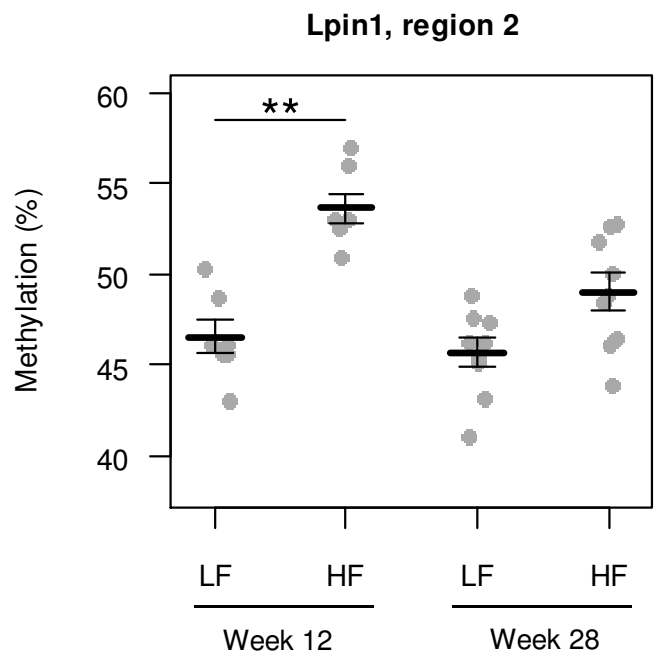

Figure 5. Effect of prenatal HF diet on hepatic DNA methylation of Lpin1 TF binding region 2 (Chr12: 16,590,890$16,591,130 \mathrm{bp}$ ) at 12 weeks ( $\mathrm{n}=7$ per group) and 28 weeks of age ( $n=9$ per group). Lines and error bars indicate mean \pm SEM. **FDR q-value $<0.01$, based on twotailed independent samples t-test followed by correction for multiple testing using the Benjamini-Hochberg procedure.

\section{mRNA expression of Lpin1 correlates with DNA methylation of Lpin1 TF binding sites}

The observed hypermethylation of Lpin1 TF binding region 2 in 12 week old HF/HF offspring was concurrent with a decrease in mRNA expression of Lpin1, indicating a negative relation between DNA methylation and gene expression for this locus. Existence of a correlation, however, would provide a stronger indication for DNA methylation to be involved in regulation of gene expression. Therefore, Pearson correlation was assessed for mean DNA methylation levels of TF binding regions versus microarray expression levels of the corresponding gene. This analysis showed that at 12 weeks of age, Lpin 1 mRNA expression correlated significantly with DNA methylation of Lpin1 TF binding region 2 ( $r=-0.54$, $p<0.05)$. Furthermore, Saa1 mRNA expression and DNA methylation of Saa1 TF binding region 1 also correlated significantly $(r=0.53 ; p<0.05)$. At 28 weeks of age, both these correlations were no longer significant. However, there was a significant correlation between Lpin1 mRNA expression and DNA methylation of Lpin1 TF binding region 3 ( $r=$ $0.50, p<0.05)$ at 28 weeks of age. Interestingly, this Lpin 1 region 3 is a reported binding site for both the TFs Nrf2 and SREBP-1 (obtained from GTRD, v. 17.04).

\section{Discussion}

While the phenotypic effects of a prenatal HF diet on offspring have been studied thoroughly, it is less well known through which mechanisms these long lasting effects are transmitted. Therefore, we studied the effect of a combined paternal and maternal HF diet on hepatic DNA methylation and gene expression in adult mouse offspring. We showed that 
this prenatal HF diet altered hepatic DNA methylation and gene expression of pathways involved in lipid metabolism, oxidative stress response and cell proliferation. We hypothesize that these changes underlie the previously reported postprandial hypertriglyceridemia and decreased hepatic lipid clearance in offspring exposed to a prenatal HF diet [24].

First, we showed that a prenatal HF diet consistently downregulated the gene set Activation of gene expression by SREBP at both 12 and 28 weeks of age. SREBP (Sterol regulatory element-binding protein) represents a group of TFs that regulate expression of genes involved in fatty acid and cholesterol synthesis and uptake [39-44]. Since SREBP targets are involved in lipid uptake, the observed downregulation of hepatic SREBP signaling in HF/HF offspring may contribute to the impaired postprandial hepatic lipid clearance, as was previously reported in these offspring mice [24]. The observed downregulation of SREBP signaling is in agreement with two previous studies which examined the effect of a maternal HF diet on offspring liver. These studies reported that SREBP gene and protein expression were diminished and that this regulated the observed decrease in mRNA expression of genes involved in fatty acid, cholesterol and steroid biosynthesis, and other SREBP-related functions $[45,46]$. In contrast, two other studies found an increase in mRNA expression of Srebp1c and some of its downstream genes involved in de novo lipogenesis in response to a prenatal HF diet $[13,14]$. Although the reasons for these opposite effects on SREBP expression in similar experimental settings remain unclear, these reports do underline the involvement of SREBP in the effects of a prenatal HF diet on offspring liver.

Next to a decreased hepatic lipid uptake induced by SREBP downregulation, alterations in the metabolic phenotype of HF/HF offspring can also be a result of an increased export of lipids from the liver into the circulation. Such an increased export may be facilitated by the upregulation of the pathway Kinesins in HF/HF offspring. Kinesins are motor proteins that are, amongst other processes, required for intracellular transport of TG-rich lipid droplets to the smooth endoplasmic reticulum in hepatocytes [47]. This transport enables lipid droplets to be catabolized and TGs to be available for VLDL assembly and subsequent secretion from the liver. Upregulation of these kinesin motors could therefore contribute to enhanced VLDL-mediated export of lipids from the liver to the blood, thereby adding to the previously reported trend for decreased fasted hepatic lipid content in HF/HF offspring [24].

Besides the Kinesins pathway, the prenatal HF diet also increased the expression of the gene set Nrf2 targets in 12 weeks old offspring. Nrf2 (Nuclear factor erythroid-derived 2-like 2) is a TF that regulates expression of antioxidant proteins that protect against oxidative damage triggered by injury and inflammation[48-50]. To examine whether these mice also displayed 
increased levels of oxidative stress, intracellular levels of oxidized and reduced glutathione were measured in offspring livers. This showed that at 12 weeks of age, oxidative stress levels were indeed significantly increased in HF/HF offspring liver compared to LF/HF. At 28 weeks of age, however, oxidized and reduced glutathione levels, as well as expression of the Nrf2 pathway, were no longer different between HF/HF and LF/HF offspring. Instead, both groups displayed elevated levels of oxidative stress, similar to the levels observed in 12 weeks old HF/HF offspring. These findings suggest that the post-weaning HF diet led to a gradual increase in oxidative stress levels in livers, irrespective of the parent's diet. If the parents were additionally exposed to a HF diet, the increased levels of oxidative stress were already reached at 12 weeks of age, thereby prolonging the time that offspring livers were exposed to oxidative stress. This link between a HF diet and oxidative stress response has been indicated before by studies showing that pharmacologic or transgenic activation of Nrf2 protects mice from the effects of a HF diet, such as obesity, insulin resistance and liver steatosis [51,52]. Furthermore, it has previously been reported that exposure to a prenatal HF diet affected not only hepatic mRNA levels [17,53], but also DNA methylation of Nrf2related genes [17], implying that epigenetics may regulate the effects of a prenatal HF diet on oxidative stress response.

To further investigate the involvement of epigenetics in the effects of a prenatal HF diet, DNA methylation was measured at $\mathrm{CpGs}$ within TF binding sites of selected differentially expressed genes. We found that the prenatal HF diet hypermethylated a TF binding region upstream of Lpin1. In addition, DNA methylation of two Lpin1 TF binding regions correlated significantly with Lpin1 gene expression. The observed correlations suggest that in current study Lpin1 mRNA expression was (at least partly) regulated by DNA methylation. The lipin 1 protein has several biological functions, depending on the tissue in which it is expressed [54-56]. In liver, lipin 1 mainly functions as a transcriptional co-activator and its knockdown leads to increased VLDL secretion and elevated plasma TGs [55]. Therefore, hypermethylation of Lpin1 TF binding regions and subsequent downregulation of Lpin1 expression may explain the phenotypic changes in HF/HF offspring, including increased circulatory TGs and decreased hepatic lipids.

In addition to Lpin 1, a correlation was also observed between a TF binding region and mRNA expression for Saa1. Saa1 is an acute phase protein mainly produced by hepatocytes in response to inflammation and tissue damage. Normally, the liver is able to recover from tissue damage [57-59]. In the current study, however, pathway analysis of microarray data showed that the prenatal HF diet downregulated expression of various gene sets involved in mitosis, cell cycle, proteasomal degradation, Wnt signaling and Hedgehog signaling in offspring liver. Although we do not have phenotypical data to further support this, these results are an indication that cell proliferation is reduced in HF/HF offspring, possibly leading 
to impaired recovery from liver damage. These findings are in line with a previous study which reported that rat offspring of mothers fed a HF diet showed hepatic cell cycle inhibition, together with related changes in gene expression and DNA methylation [60]. Similarly, many genes involved in cell growth, differentiation, proliferation and development were both differentially expressed and differentially methylated in livers of offspring of HF diet-fed mothers [17]. Furthermore, it has been shown that exposure to HF diet not only before birth but also after birth results in decreased liver proliferation, indicated by impaired liver regeneration after partial hepatectomy in mice $[61,62]$. Therefore, alterations in tissue damage and cell proliferation in liver after a prenatal HF diet deserve further attention in future research.

In comparison to previous reports, our study has a few advantages. Firstly, whereas most studies only examined the effects of a prenatal HF diet at one specific offspring age, we performed measurements in offspring at 12 and 28 weeks of age. This enabled us to analyze alterations in gene expression and DNA methylation over time. Secondly, many studies only focus either on the phenotypic outcome of a prenatal HF diet or on mechanisms underlying these effects. In our experiment, previously published effects on the metabolic phenotype of offspring were now linked to alterations in gene expression, DNA methylation and oxidative stress. Finally, most studies examine the effects of a prenatal HF diet by combining a maternal HF diet with a paternal LF diet or vice versa. However, in our study both father and mother were fed the same diet to better mimic the current Western dietary situation in which family members share eating patterns [19]. In addition, combining a maternal and paternal HF diet can have additive effects on the offspring's metabolic health, resulting in more prominent health effects in offspring when compared to separate maternal or paternal HF diets $[14,16]$.

With the current study design, we did not assess the effect of a post-weaning LF diet. All offspring was weaned onto a HF diet, since this experimental setup provides a better model for the current Western type of diet, which is characterized by a HF content. Furthermore, it has been shown that a post-weaning HF diet may aggravate the negative effects of the prenatal HF diet [15]. Another study limitation is that the analyses were limited to male offspring. Sex differences occur in response to a prenatal HF diet, with male offspring having a larger increase in bodyweight and plasma TG [23], whereas female offspring have higher blood pressure and leptin levels $[20,21]$. Likewise, exposure to a prenatal HF diet differently affects hepatic gene expression in male and female offspring [23]. Major contributors to these sex dimorphisms are estrogens and androgens $[63,64]$. In females, these effects depend on the hormonal cycle, making female mice more prone to variation in metabolic outcomes. Therefore, only male offspring were included in present study. 
In summary, we showed that a combined maternal and paternal HF diet alters hepatic gene expression of pathways related to lipid synthesis and uptake, oxidative stress response, and cell proliferation. The downregulation of lipid uptake could account for the previously observed decreased postprandial hepatic lipid uptake and hypertriglyceridemia in $\mathrm{HF} / \mathrm{HF}$ offspring. Furthermore, the upregulation of the Nrf2-mediated oxidative stress response was confirmed by assessment of GSH/GSSG ratios in livers of offspring. In other words, the changes in gene expression due to prenatal HF diet were mechanistically linked to phenotypic alterations. We propose that the observed effects on the offspring's metabolic phenotype and gene expression are driven by alterations in DNA methylation, as indicated by the hypermethylation of TF binding sites upstream of Lpin1, and the correlation between DNA methylation of Lpin1 TF binding sites and gene expression of Lpin1. Society may have to invest in early life nutrition to prevent common diseases occurring in offspring at older ages. More knowledge is needed to unravel the molecular mechanisms, including DNA methylation and other epigenetic mechanisms, that underlie the negative effects of a prenatal HF diet on offspring health.

\section{Acknowledgements / grant support}

This work was financially supported by the Netherlands Food and Consumer Product Safety Authority (NVWA). S.R. was partially supported by the Royal Netherlands Academy of Arts and Sciences (KNAW) Ter Meulen Grant. The authors would like to thank Lou Maas and Marie-José Drittij for technical support. 


\section{References}

1. Barker, D.J. Maternal nutrition, fetal nutrition, and disease in later life. Nutrition 1997, 13, 807-813.

2. de Rooij, S.R.; Painter, R.C.; Roseboom, T.J.; Phillips, D.I.; Osmond, C.; Barker, D.J.; Tanck, M.W.; Michels, R.P.; Bossuyt, P.M.; Bleker, O.P. Glucose tolerance at age 58 and the decline of glucose tolerance in comparison with age 50 in people prenatally exposed to the Dutch famine. Diabetologia 2006, 49, 637-643, doi:10.1007/s00125-005-0136-9.

3. Ravelli, A.C.; van der Meulen, J.H.; Michels, R.P.; Osmond, C.; Barker, D.J.; Hales, C.N.; Bleker, O.P. Glucose tolerance in adults after prenatal exposure to famine. Lancet 1998, 351, 173177.

4. Ravelli, A.C.J.; van der Meulen, J.H.P.; Osmond, C.; Barker, D.J.P.; Bleker, O.P. Obesity at the age of $50 \mathrm{y}$ in men and women exposed to famine prenatally. Am J Clin Nutr 1999, 70, 811816.

5. Roseboom, T.J.; van der Meulen, J.H.; Osmond, C.; Barker, D.J.; Ravelli, A.C.; SchroederTanka, J.M.; van Montfrans, G.A.; Michels, R.P.; Bleker, O.P. Coronary heart disease after prenatal exposure to the Dutch famine, 1944-45. Heart 2000, 84, 595-598.

6. Cooper, R.; Hyppönen, E.; Berry, D.; Power, C. Associations between parental and offspring adiposity up to midlife: the contribution of adult lifestyle factors in the 1958 British Birth Cohort Study-. The American journal of clinical nutrition 2010, 92, 946-953.

7. Li, L.; Law, C.; Lo Conte, R.; Power, C. Intergenerational influences on childhood body mass index: the effect of parental body mass index trajectories-. The American journal of clinical nutrition 2008, 89, 551-557.

8. Eriksson, J.G.; Sandboge, S.; Salonen, M.K.; Kajantie, E.; Osmond, C. Long-term consequences of maternal overweight in pregnancy on offspring later health: findings from the Helsinki Birth Cohort Study. Annals of medicine 2014, 46, 434-438.

9. Gaillard, R.; Steegers, E.A.; Duijts, L.; Felix, J.F.; Hofman, A.; Franco, O.H.; Jaddoe, V.W. Childhood cardiometabolic outcomes of maternal obesity during pregnancy: the Generation R Study. Hypertension 2014, 63, 683-691.

10. Hochner, H.; Friedlander, Y.; Calderon-Margalit, R.; Meiner, V.; Sagy, Y.; Avgil-Tsadok, M.; Burger, A.; Savitsky, B.; Siscovick, D.S.; Manor, O. Associations of Maternal Prepregnancy Body Mass Index and Gestational Weight Gain With Adult Offspring Cardiometabolic Risk FactorsClinical Perspective: The Jerusalem Perinatal Family Follow-Up Study. Circulation 2012, 125, 1381-1389.

11. Yu, Z.; Han, S.; Zhu, J.; Sun, X.; Ji, C.; Guo, X. Pre-pregnancy body mass index in relation to infant birth weight and offspring overweight/obesity: a systematic review and metaanalysis. Plos One 2013, 8, e61627.

12. Keleher, M.R.; Zaidi, R.; Shah, S.; Oakley, M.E.; Pavlatos, C.; El Idrissi, S.; Xing, X.; Li, D.; Wang, T.; Cheverud, J.M. Maternal high-fat diet associated with altered gene expression, DNA methylation, and obesity risk in mouse offspring. Plos One 2018, 13, e0192606.

13. Pruis, M.; Lendvai, A.; Bloks, V.; Zwier, M.; Baller, J.; De Bruin, A.; Groen, A.; Plösch, T. Maternal western diet primes non-alcoholic fatty liver disease in adult mouse offspring. Acta Physiologica 2014, 210, 215-227. 
14. Ornellas, F.; Souza-Mello, V.; Mandarim-de-Lacerda, C.A.; Aguila, M.B. Programming of obesity and comorbidities in the progeny: lessons from a model of diet-induced obese parents. Plos One 2015, 10, e0124737.

15. Fullston, T.; McPherson, N.O.; Owens, J.A.; Kang, W.X.; Sandeman, L.Y.; Lane, M. Paternal obesity induces metabolic and sperm disturbances in male offspring that are exacerbated by their exposure to an "obesogenic" diet. Physiological reports 2015, 3, e12336.

16. Masuyama, H.; Mitsui, T.; Eguchi, T.; Tamada, S.; Hiramatsu, Y. The effects of paternal highfat diet exposure on offspring metabolism with epigenetic changes in the mouse adiponectin and leptin gene promoters. American Journal of Physiology-Endocrinology and Metabolism 2016, 311, E236-E245.

17. Seki, Y.; Suzuki, M.; Guo, X.; Glenn, A.S.; Vuguin, P.M.; Fiallo, A.; Du, Q.; Ko, Y.-A.; Yu, Y.; Susztak, K. In utero exposure to a high-fat diet programs hepatic hypermethylation and gene dysregulation and development of metabolic syndrome in male mice. Endocrinology 2017, 158, 2860-2872.

18. Soubry, A.; Murphy, S.; Wang, F.; Huang, Z.; Vidal, A.; Fuemmeler, B.; Kurtzberg, J.; Murtha, A.; Jirtle, R.; Schildkraut, J. Newborns of obese parents have altered DNA methylation patterns at imprinted genes. International journal of obesity 2015, 39, 650.

19. Berge, J.M.; Wall, M.; Larson, N.; Forsyth, A.; Bauer, K.W.; Neumark-Sztainer, D. Youth dietary intake and weight status: healthful neighborhood food environments enhance the protective role of supportive family home environments. Health \& place 2014, 26, 69-77.

20. Khan, I.; Dekou, V.; Hanson, M.; Poston, L.; Taylor, P. Predictive adaptive responses to maternal high-fat diet prevent endothelial dysfunction but not hypertension in adult rat offspring. Circulation 2004, 110, 1097-1102.

21. Bellisario, V.; Berry, A.; Capoccia, S.; Raggi, C.; Panetta, P.; Branchi, I.; Piccaro, G.; Giorgio, M.; Pelicci, P.G.; Cirulli, F. Gender-dependent resiliency to stressful and metabolic challenges following prenatal exposure to high-fat diet in the p66Shc-/- mouse. Frontiers in behavioral neuroscience 2014, 8, 285.

22. Miller, C.; Krishna, S.; Lin, Z.; Della-Fera, M.A.; Harn, D.; de la Serre, C.; Baile, C.; Filipov, N. Early sex differences in hepatic metabolic signaling in offspring of obese female mice (1033.11). The FASEB Journal 2014, 28, 1033.1011.

23. Mischke, M.; Pruis, M.G.; Boekschoten, M.V.; Groen, A.K.; Fitri, A.R.; van de Heijning, B.J.; Verkade, H.J.; Müller, M.; Plösch, T.; Steegenga, W.T. Maternal Western-style high fat diet induces sex-specific physiological and molecular changes in two-week-old mouse offspring. Plos One 2013, 8, e78623.

24. van Ewijk, P.A.; Paglialunga, S.; Kooi, M.E.; Nunes, P.M.; Gemmink, A.; Slenter, J.; Kornips, E.; Jorgensen, J.A.; Hoeks, J.; Wildberger, J.E.; et al. Effects of high-fat feeding on ectopic fat storage and postprandial lipid metabolism in mouse offspring. Obesity (Silver Spring) 2015, 23, 2242-2250, doi:10.1002/oby.21235.

25. Lin, K.; Kools, H.; de Groot, P.J.; Gavai, A.K.; Basnet, R.K.; Cheng, F.; Wu, J.; Wang, X.; Lommen, A.; Hooiveld, G.J. MADMAX-Management and analysis database for multiple omics experiments. Journal of integrative bioinformatics 2011, 8, 59-74. 
26. Bolstad, B.M.; Irizarry, R.A.; Åstrand, M.; Speed, T.P. A comparison of normalization methods for high density oligonucleotide array data based on variance and bias. Bioinformatics 2003, 19, 185-193.

27. Subramanian, A.; Tamayo, P.; Mootha, V.K.; Mukherjee, S.; Ebert, B.L.; Gillette, M.A.; Paulovich, A.; Pomeroy, S.L.; Golub, T.R.; Lander, E.S. Gene set enrichment analysis: a knowledge-based approach for interpreting genome-wide expression profiles. Proceedings of the National Academy of Sciences 2005, 102, 15545-15550.

28. Nishimura, D. BioCarta. Biotech Software \& Internet Report: The Computer Software Journal for Scient 2001, 2, 117-120.

29. Kanehisa, M.; Goto, S. KEGG: kyoto encyclopedia of genes and genomes. Nucleic acids research 2000, 28, 27-30.

30. Fabregat, A.; Sidiropoulos, K.; Garapati, P.; Gillespie, M.; Hausmann, K.; Haw, R.; Jassal, B.; Jupe, S.; Korninger, F.; McKay, S. The reactome pathway knowledgebase. Nucleic acids research 2015, 44, D481-D487.

31. Slenter, D.N.; Kutmon, M.; Hanspers, K.; Riutta, A.; Windsor, J.; Nunes, N.; Mélius, J.; Cirillo, E.; Coort, S.L.; Digles, D. WikiPathways: a multifaceted pathway database bridging metabolomics to other omics research. Nucleic acids research 2017, 46, D661-D667.

32. Sartor, M.A.; Tomlinson, C.R.; Wesselkamper, S.C.; Sivaganesan, S.; Leikauf, G.D.; Medvedovic, M. Intensity-based hierarchical Bayes method improves testing for differentially expressed genes in microarray experiments. BMC bioinformatics 2006, 7, 538.

33. Wang, X.; Seed, B. A PCR primer bank for quantitative gene expression analysis. Nucleic acids research 2003, 31, e154-e154.

34. Cui, W.; Taub, D.D.; Gardner, K. qPrimerDepot: a primer database for quantitative real time PCR. Nucleic acids research 2006, 35, D805-D809.

35. Livak, K.J.; Schmittgen, T.D. Analysis of relative gene expression data using real-time quantitative PCR and the 2- $\triangle \Delta C T$ method. methods 2001, 25, 402-408.

36. Yevshin, I.; Sharipov, R.; Valeev, T.; Kel, A.; Kolpakov, F. GTRD: a database of transcription factor binding sites identified by ChIP-seq experiments. Nucleic acids research 2016, gkw951.

37. Rahman, I.; Kode, A.; Biswas, S.K. Assay for quantitative determination of glutathione and glutathione disulfide levels using enzymatic recycling method. Nat Protoc 2006, 1, 31593165, doi:10.1038/nprot.2006.378.

38. Benjamini, Y.; Hochberg, Y. Controlling the false discovery rate: a practical and powerful approach to multiple testing. Journal of the Royal statistical society: series $B$ (Methodological) 1995, 57, 289-300.

39. Horton, J.D.; Goldstein, J.L.; Brown, M.S. SREBPs: activators of the complete program of cholesterol and fatty acid synthesis in the liver. The Journal of clinical investigation 2002, $109,1125-1131$.

40. Sakakura, Y.; Shimano, H.; Sone, H.; Takahashi, A.; Inoue, K.; Toyoshima, H.; Suzuki, S.; Yamada, N. Sterol regulatory element-binding proteins induce an entire pathway of cholesterol synthesis. Biochem Bioph Res Co 2001, 286, 176-183.

41. Shimano, H.; Horton, J.D.; Hammer, R.E.; Shimomura, I.; Brown, M.S.; Goldstein, J.L. Overproduction of cholesterol and fatty acids causes massive liver enlargement in 
transgenic mice expressing truncated SREBP-1a. The Journal of clinical investigation 1996, 98, 1575-1584.

42. Shimano, H.; Horton, J.D.; Shimomura, I.; Hammer, R.E.; Brown, M.S.; Goldstein, J.L. Isoform $1 \mathrm{c}$ of sterol regulatory element binding protein is less active than isoform $1 \mathrm{a}$ in livers of transgenic mice and in cultured cells. The Journal of clinical investigation 1997, 99, 846-854.

43. Shimano, H.; Shimomura, I.; Hammer, R.E.; Herz, J.; Goldstein, J.L.; Brown, M.S.; Horton, J.D. Elevated levels of SREBP-2 and cholesterol synthesis in livers of mice homozygous for a targeted disruption of the SREBP-1 gene. The Journal of clinical investigation 1997, 100, 2115-2124.

44. Horton, J.D.; Shimomura, I.; Brown, M.S.; Hammer, R.E.; Goldstein, J.L.; Shimano, H. Activation of cholesterol synthesis in preference to fatty acid synthesis in liver and adipose tissue of transgenic mice overproducing sterol regulatory element-binding protein-2. The Journal of clinical investigation 1998, 101, 2331-2339.

45. Yu, H.-L.; Miao, H.-T.; Gao, L.-F.; Li, L.; Xi, Y.-D.; Nie, S.-P.; Xiao, R. Adaptive responses by mouse fetus to a maternal high lipid and high energy diet by down-regulating SREBP1: a microarray-and bio-analytic-based study. Journal of lipid research 2013, jlr. M037416.

46. Cannon, M.V.; Buchner, D.A.; Hester, J.; Miller, H.; Sehayek, E.; Nadeau, J.H.; Serre, D. Maternal nutrition induces pervasive gene expression changes but no detectable DNA methylation differences in the liver of adult offspring. Plos One 2014, 9, e90335.

47. Rai, P.; Kumar, M.; Sharma, G.; Barak, P.; Das, S.; Kamat, S.S.; Mallik, R. Kinesin-dependent mechanism for controlling triglyceride secretion from the liver. Proceedings of the National Academy of Sciences 2017, 114, 12958-12963.

48. Yamamoto, M.; Kensler, T.W.; Motohashi, H. The KEAP1-NRF2 system: a thiol-based sensoreffector apparatus for maintaining redox homeostasis. Physiological reviews 2018, 98, 11691203.

49. Itoh, K.; Chiba, T.; Takahashi, S.; Ishii, T.; Igarashi, K.; Katoh, Y.; Oyake, T.; Hayashi, N.; Satoh, K.; Hatayama, I. An Nrf2/small Maf heterodimer mediates the induction of phase II detoxifying enzyme genes through antioxidant response elements. Biochem Bioph Res Co 1997, 236, 313-322.

50. Itoh, K.; Wakabayashi, N.; Katoh, Y.; Ishii, T.; Igarashi, K.; Engel, J.D.; Yamamoto, M. Keap1 represses nuclear activation of antioxidant responsive elements by Nrf2 through binding to the amino-terminal Neh2 domain. Genes \& development 1999, 13, 76-86.

51. Shin, S.; Wakabayashi, J.; Yates, M.S.; Wakabayashi, N.; Dolan, P.M.; Aja, S.; Liby, K.T.; Sporn, M.B.; Yamamoto, M.; Kensler, T.W. Role of Nrf2 in prevention of high-fat diet-induced obesity by synthetic triterpenoid CDDO-imidazolide. European journal of pharmacology 2009, 620, 138-144.

52. Slocum, S.L.; Skoko, J.J.; Wakabayashi, N.; Aja, S.; Yamamoto, M.; Kensler, T.W.; Chartoumpekis, D.V. Keap1/Nrf2 pathway activation leads to a repressed hepatic gluconeogenic and lipogenic program in mice on a high-fat diet. Archives of biochemistry and biophysics 2016, 591, 57-65.

53. Terashima, M.; Barbour, S.; Ren, J.; Yu, W.; Han, Y.; Muegge, K. Effect of high fat diet on paternal sperm histone distribution and male offspring liver gene expression. Epigenetics 2015, 10, 861-871. 
54. Chen, Y.; Rui, B.-B.; Tang, L.-Y.; Hu, C.-M. Lipin family proteins-key regulators in lipid metabolism. Annals of Nutrition and Metabolism 2015, 66, 10-18.

55. Finck, B.N.; Gropler, M.C.; Chen, Z.; Leone, T.C.; Croce, M.A.; Harris, T.E.; Lawrence, J.C.; Kelly, D.P. Lipin 1 is an inducible amplifier of the hepatic PGC-1 $\alpha /$ PPAR $\alpha$ regulatory pathway. Cell metabolism 2006, 4, 199-210.

56. Reue, K.; Brindley, D.N. Thematic Review Series: Glycerolipids. Multiple roles for lipins/phosphatidate phosphatase enzymes in lipid metabolism. Journal of lipid research 2008, 49, 2493-2503.

57. Fausto, N.; Campbell, J.S.; Riehle, K.J. Liver regeneration. Hepatology 2006, 43, S45-S53.

58. Michalopoulos, G.K. Liver regeneration. Journal of cellular physiology 2007, 213, 286-300.

59. Taub, R. Liver regeneration: from myth to mechanism. Nature reviews Molecular cell biology 2004, 5, 836.

60. Dudley, K.J.; Sloboda, D.M.; Connor, K.L.; Beltrand, J.; Vickers, M.H. Offspring of mothers fed a high fat diet display hepatic cell cycle inhibition and associated changes in gene expression and DNA methylation. Plos One 2011, 6, e21662.

61. DeAngelis, R.A.; Markiewski, M.M.; Taub, R.; Lambris, J.D. A high-fat diet impairs liver regeneration in C57BL/6 mice through overexpression of the NF-KB inhibitor, IKB $\alpha$. Hepatology 2005, 42, 1148-1157.

62. Yang, S.Q.; Lin, H.Z.; Mandal, A.K.; Huang, J.; Diehl, A.M. Disrupted signaling and inhibited regeneration in obese mice with fatty livers: implications for nonalcoholic fatty liver disease pathophysiology. Hepatology 2001, 34, 694-706.

63. Mauvais-Jarvis, F.; Clegg, D.J.; Hevener, A.L. The role of estrogens in control of energy balance and glucose homeostasis. Endocrine reviews 2013, 34, 309-338.

64. Navarro, G.; Allard, C.; Xu, W.; Mauvais-Jarvis, F. The role of androgens in metabolism, obesity, and diabetes in males and females. Obesity 2015, 23, 713-719. 


\section{Supplement}

Table S1. Real time qPCR primers.

\begin{tabular}{|c|c|c|}
\hline Gene & Primer & Sequence \\
\hline \multirow[t]{2}{*}{ Cd163 } & Forward & GGTGGACACAGAATGGTTCTTC \\
\hline & Reverse & CCAGGAGCGTTAGTGACAGC \\
\hline \multirow[t]{2}{*}{ Hmgcr } & Forward & TGTTCACCGGCAACAACAAGA \\
\hline & Reverse & CCGCGTTATCGTCAGGATGA \\
\hline \multirow[t]{2}{*}{ Aacs } & Forward & GTGGAATCGTCTACTCACGCA \\
\hline & Reverse & TAAAGGGCGACTCTGTCGTTC \\
\hline \multirow[t]{2}{*}{ Lpin1 } & Forward & AAGAGACTGACAACGATCAGGA \\
\hline & Reverse & TTCCCCAGAGAACCAGTGGAT \\
\hline \multirow[t]{2}{*}{ Pla2g16 } & Forward & GTCTGCTTTGACTGACAAGGC \\
\hline & Reverse & GTGTACTCCTCGTCATGTTTGTT \\
\hline \multirow[t]{2}{*}{ Saa1 } & Forward & TCATGTCAGTGTAGGCTCGC \\
\hline & Reverse & CCAGGAGACACCAGGATGAA \\
\hline \multirow[t]{2}{*}{$\| 1 r 1$} & Forward & GTGCTACTGGGGCTCATTTGT \\
\hline & Reverse & GGAGTAAGAGGACACTTGCGAAT \\
\hline \multirow[t]{2}{*}{ Gapdh } & Forward & TTGATGGCAACAATCTCCAC \\
\hline & Reverse & CGTCCCGTAGACAAAATGGT \\
\hline \multirow[t]{2}{*}{ Actb } & Forward & ATGGAGGGGAATACAGCCC \\
\hline & Reverse & TTCTTTGCAGCTCCTTCGTT \\
\hline
\end{tabular}




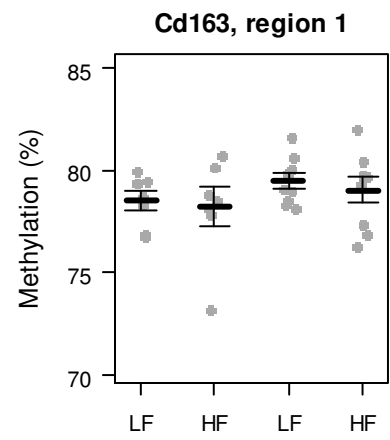

Week 12 Week 28

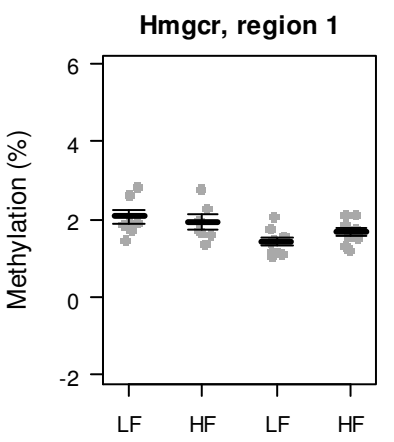

Week 12 Week 28

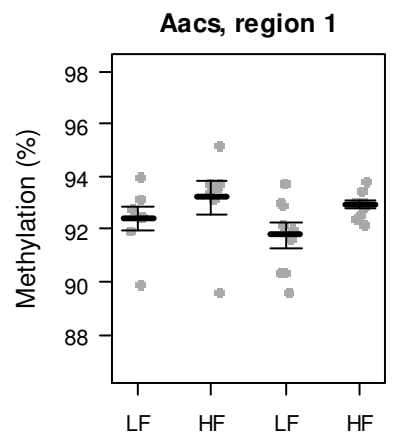

Week 12 Week 28

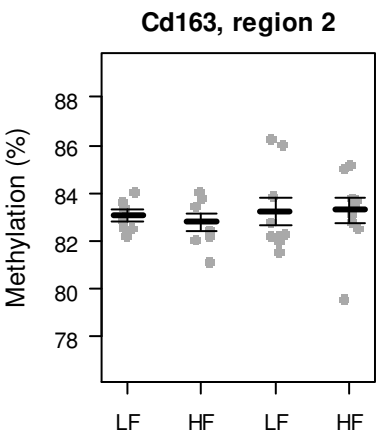

Week 12 Week 28

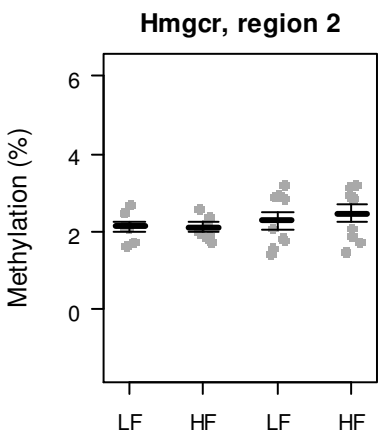

Week 12 Week 28

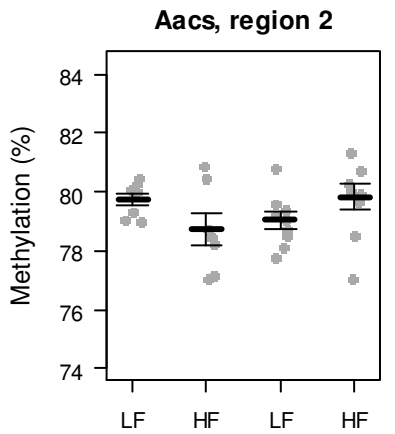

Week $12 \quad$ Week 28

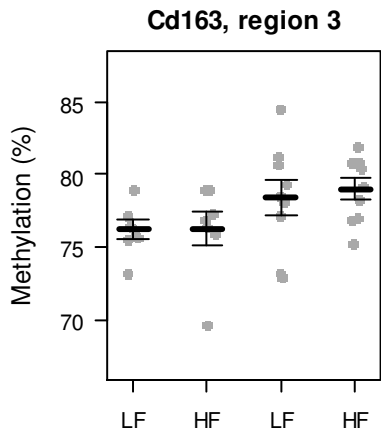

Week 12 Week 28

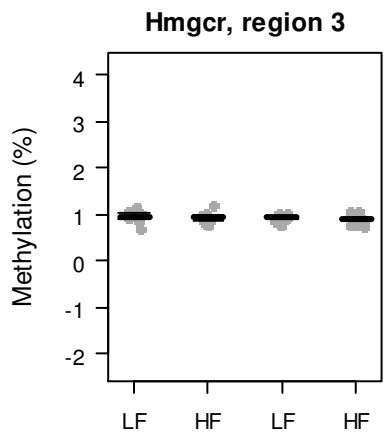

Week 12 Week 28

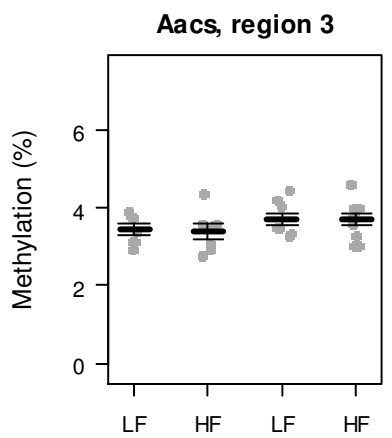

Week 12 Week 28 


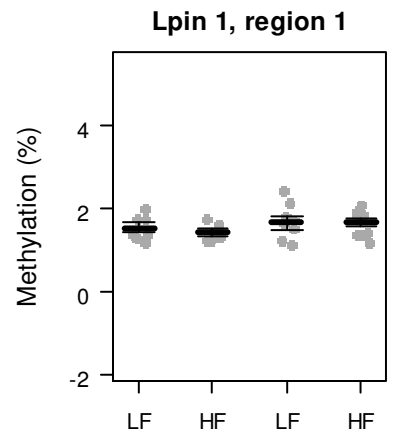

Week 12 Week 28

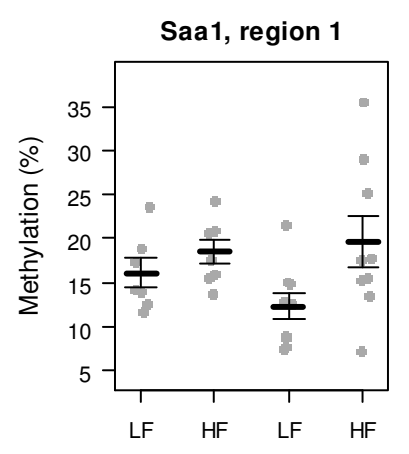

Week 12 Week 28

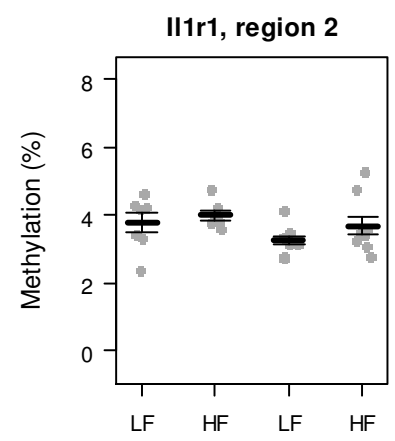

Week 12 Week 28

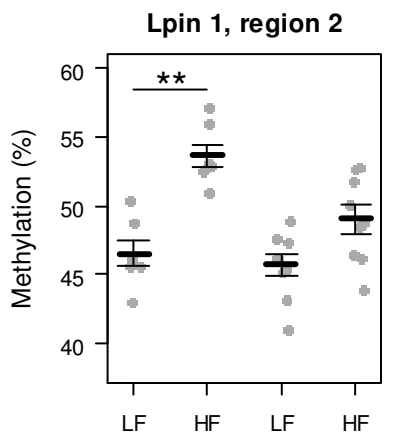

Week 12 Week 28

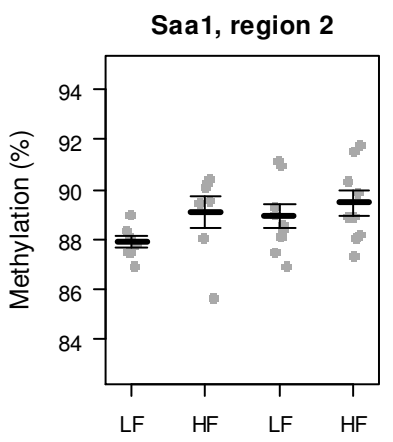

Week 12 Week 28

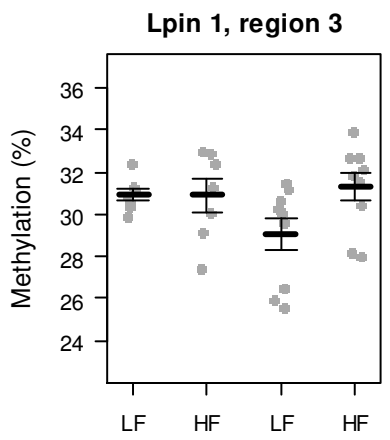

Week 12 Week 28

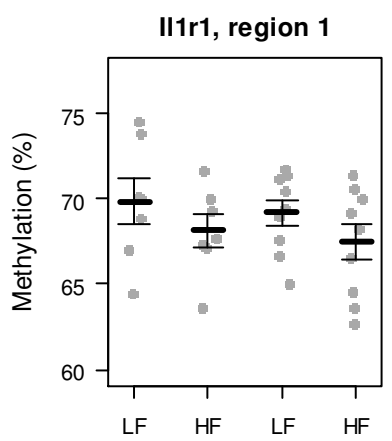

Week 12 Week 28

Figure S1. Effect of prenatal HF diet on offspring hepatic DNA methylation of TF binding regions upstream of $C d 163$, Hmgcr, Aacs, Lpin1, Saa1 and IIr1 at 12 weeks ( $n=7$ per group) and 28 weeks of age ( $n=9$ per group). Lines and error bars indicate mean \pm SEM. ${ }^{* *}$ FDR q-value $<0.01$. 
Table S2. A complete overview of all pathways significantly affected by prenatal HF diet in offspring liver at 12 weeks ( $n=7$ per group) and 28 weeks ( $n=9$ per group) of age. A negative or positive NES indicates that the pathway was downregulated or upregulated, respectively, in $\mathrm{HF} / \mathrm{HF}$ offspring

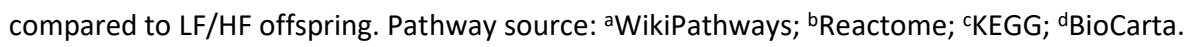

\begin{tabular}{|c|c|c|c|}
\hline \multicolumn{4}{|c|}{12 weeks } \\
\hline Biological function of pathway & Pathway & NES & FDR \\
\hline Cellular movement & Striated muscle contraction ${ }^{a}$ & -2.34 & 0.004 \\
\hline Lipid biosynthesis / uptake & Activation of gene expression by SREBPb & -2.32 & 0.002 \\
\hline Oxidative stress response & Nrf2 targets ${ }^{\mathrm{b}}$ & 2.60 & $<0.000$ \\
\hline Intracellular transport & Kinesins $^{b}$ & 2.08 & 0.015 \\
\hline
\end{tabular}

\begin{tabular}{|c|c|c|c|}
\hline \multicolumn{4}{|c|}{28 weeks } \\
\hline Biological function of pathway & Pathway & NES & FDR \\
\hline \multirow{3}{*}{ Lipid biosynthesis / uptake } & Cholesterol biosynthesis $^{b}$ & -2.29 & 0.002 \\
\hline & Activation of gene expression by SREBPb & -1.95 & 0.006 \\
\hline & Steroid biosynthesis ${ }^{c}$ & -1.91 & 0.009 \\
\hline \multirow[t]{4}{*}{ Mitochondrial translation } & Mitochondrial translation initiation ${ }^{b}$ & -2.28 & 0.001 \\
\hline & Mitochondrial translation elongation ${ }^{b}$ & -2.28 & 0.001 \\
\hline & Mitochondrial translation termination ${ }^{b}$ & -2.20 & 0.001 \\
\hline & Mitochondrial translation ${ }^{b}$ & -2.20 & 0.001 \\
\hline \multirow[t]{3}{*}{ Antigen processing / presentation } & $\begin{array}{l}\text { Cross-presentation of soluble exogenous } \\
\text { antigens (endosomes) }^{b}\end{array}$ & -2.22 & 0.001 \\
\hline & Antigen processing-Cross presentation ${ }^{b}$ & -1.77 & 0.028 \\
\hline & ER-Phagosome pathwayb & -1.84 & 0.016 \\
\hline \multirow{16}{*}{ Mitosis / Cell cycle } & Autodegradation of $\mathrm{Cdh} 1$ by $\mathrm{Cdh} 1: \mathrm{APC} / \mathrm{C}^{\mathrm{b}}$ & -2.20 & 0.001 \\
\hline & $\begin{array}{l}\text { APC/C:Cdc } 20 \text { mediated degradation of mitotic } \\
\text { proteins }^{b}\end{array}$ & -2.18 & 0.001 \\
\hline & $\begin{array}{l}\text { APC/C:Cdc } 20 \text { mediated degradation of } \\
\text { Securin }^{b}\end{array}$ & -2.18 & 0.001 \\
\hline & $\begin{array}{l}\text { Activation of } \mathrm{APC} / \mathrm{C} \text { and } \mathrm{APC} / \mathrm{C}: \mathrm{Cdc} 20 \\
\text { mediated degradation of mitotic proteins }\end{array}$ & -2.16 & 0.001 \\
\hline & $\begin{array}{l}\text { Cdc20:Phospho-APC/C mediated degradation } \\
\text { of Cyclin } A^{b}\end{array}$ & -2.14 & 0.001 \\
\hline & $\begin{array}{l}\text { APC/C:Cdh1 mediated degradation of } \mathrm{Cdc} 20 \\
\text { and other APC/C:Cdh1 targeted proteins in } \\
\text { late mitosis/early } \mathrm{G} 1^{\mathrm{b}}\end{array}$ & -2.14 & 0.001 \\
\hline & Stabilization of $\mathrm{p} 53^{\mathrm{b}}$ & -2.11 & 0.001 \\
\hline & $\begin{array}{l}\text { Autodegradation of the E3 ubiquitin ligase } \\
\text { COP } 1^{b}\end{array}$ & -2.10 & 0.001 \\
\hline & $\begin{array}{l}\text { Regulation of activated PAK- } 2 \text { p34 by } \\
\text { proteasome mediated degradation }^{\text {b }}\end{array}$ & -2.06 & 0.002 \\
\hline & $\begin{array}{l}\text { CDK-mediated phosphorylation and removal } \\
\text { of } C d c 6^{b}\end{array}$ & -2.01 & 0.004 \\
\hline & Regulation of apoptosis ${ }^{b}$ & -1.99 & 0.005 \\
\hline & 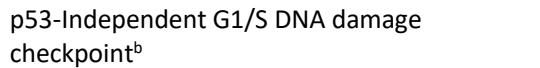 & -1.99 & 0.005 \\
\hline & $\begin{array}{l}\text { Ubiquitin Mediated Degradation of } \\
\text { Phosphorylated Cdc } 25 \mathrm{~A}^{\mathrm{b}}\end{array}$ & -1.98 & 0.005 \\
\hline & P53 independent DNA damage response ${ }^{b}$ & -1.98 & 0.005 \\
\hline & $\begin{array}{l}\text { P53 dependent G1/S DNA damage } \\
\text { checkpoint }^{\text {b }}\end{array}$ & -1.98 & 0.005 \\
\hline & SCF(Skp2)-mediated degradation of $\mathrm{p} 27 / \mathrm{p} 21^{\mathrm{b}}$ & -1.97 & 0.005 \\
\hline
\end{tabular}


Proteasomal degradation

HIV-host interaction

Regulation of transcription and translation

Wnt signaling

Hedgehog signaling

Sema4D signaling

\begin{tabular}{|c|c|c|}
\hline $\begin{array}{l}\text { Ubiquitin-dependent degradation of Cyclin } \\
\text { D1 }\end{array}$ & -1.97 & 0.005 \\
\hline P53 dependent G1 DNA damage response $\mathrm{b}^{\mathrm{b}}$ & -1.97 & 0.005 \\
\hline $\begin{array}{l}\text { APC/C-mediated degradation of cell cycle } \\
\text { proteins }^{b}\end{array}$ & -1.96 & 0.006 \\
\hline Regulation of mitotic cell cycle ${ }^{b}$ & -1.95 & 0.006 \\
\hline $\begin{array}{l}\text { Ubiquitin-dependent degradation of Cyclin } \\
\text { D1 }{ }^{\text {b }}\end{array}$ & -1.95 & 0.006 \\
\hline $\begin{array}{l}\text { Regulation of APC/C activators between G1/S } \\
\text { and early anaphase }{ }^{b}\end{array}$ & -1.94 & 0.007 \\
\hline $\begin{array}{l}\text { CDT1 association with the CDC6:ORC:origin } \\
\text { complex }^{\text {b }}\end{array}$ & -1.91 & 0.009 \\
\hline $\begin{array}{l}\text { Cyclin A:Cdk2-associated events at S phase } \\
\text { entry }^{b}\end{array}$ & -1.90 & 0.009 \\
\hline G1/S DNA damage checkpoints ${ }^{b}$ & -1.90 & 0.009 \\
\hline $\begin{array}{l}\text { Cyclin E associated events during G1/S } \\
\text { transition }^{b}\end{array}$ & -1.84 & 0.017 \\
\hline Regulation of ornithine decarboxylase $(O D C)^{b}$ & -1.80 & 0.023 \\
\hline SCF-beta-TrCP mediated degradation of Emi $1^{b}$ & -1.75 & 0.033 \\
\hline Assembly of the pre-replicative complex ${ }^{b}$ & -1.74 & 0.034 \\
\hline Proteasome ${ }^{c}$ & -2.15 & 0.001 \\
\hline Proteasome pathway ${ }^{d}$ & -2.11 & 0.001 \\
\hline Proteasome degradation ${ }^{a}$ & -1.99 & 0.005 \\
\hline Vpu mediated degradation of $C D 4^{b}$ & -1.93 & 0.008 \\
\hline Vif-mediated degradation of $A P O B E C 3 G^{b}$ & -1.93 & 0.008 \\
\hline Ribosome biogenesis in eukaryotes ${ }^{c}$ & -2.00 & 0.005 \\
\hline Eukaryotic transcription initiation ${ }^{a}$ & -1.91 & 0.009 \\
\hline RNA transport ${ }^{c}$ & -1.89 & 0.011 \\
\hline $\begin{array}{l}\text { AUF1 (hnRNP D0) binds and destabilizes } \\
\text { mRNA }^{\text {b }}\end{array}$ & -1.85 & 0.015 \\
\hline Ribosome $^{c}$ & -1.83 & 0.018 \\
\hline Basal transcription factors ${ }^{c}$ & -1.75 & 0.033 \\
\hline $\begin{array}{l}\text { Regulation of mRNA stability by proteins that } \\
\text { bind AU-rich elements }{ }^{b}\end{array}$ & -1.75 & 0.033 \\
\hline Degradation of axin ${ }^{b}$ & -1.89 & 0.011 \\
\hline Asymmetric localization of PCP proteins ${ }^{b}$ & -1.88 & 0.011 \\
\hline Degradation of DVL ${ }^{b}$ & -1.80 & 0.022 \\
\hline Hedgehog ligand biogenesis disease ${ }^{b}$ & -1.80 & 0.022 \\
\hline Degradation of GLI1 by the proteasome ${ }^{b}$ & -1.79 & 0.023 \\
\hline $\begin{array}{l}\text { Processing defective Hh variants are degraded } \\
\text { by the proteasome }{ }^{b}\end{array}$ & -1.78 & 0.027 \\
\hline Hedgehog ligand biogenesis ${ }^{b}$ & -1.76 & 0.033 \\
\hline Degradation of GLI2 by the proteasome ${ }^{b}$ & -1.75 & 0.033 \\
\hline $\begin{array}{l}\text { GLI3 is processed to GLI3R by the } \\
\text { proteasome }^{\text {b }}\end{array}$ & -1.72 & 0.040 \\
\hline Sema4D in semaphorin signaling ${ }^{b}$ & 2.11 & 0.040 \\
\hline $\begin{array}{l}\text { Sema4D-induced cell migration and growth } \\
\text { cone collapse }^{b}\end{array}$ & 2.06 & 0.038 \\
\hline
\end{tabular}




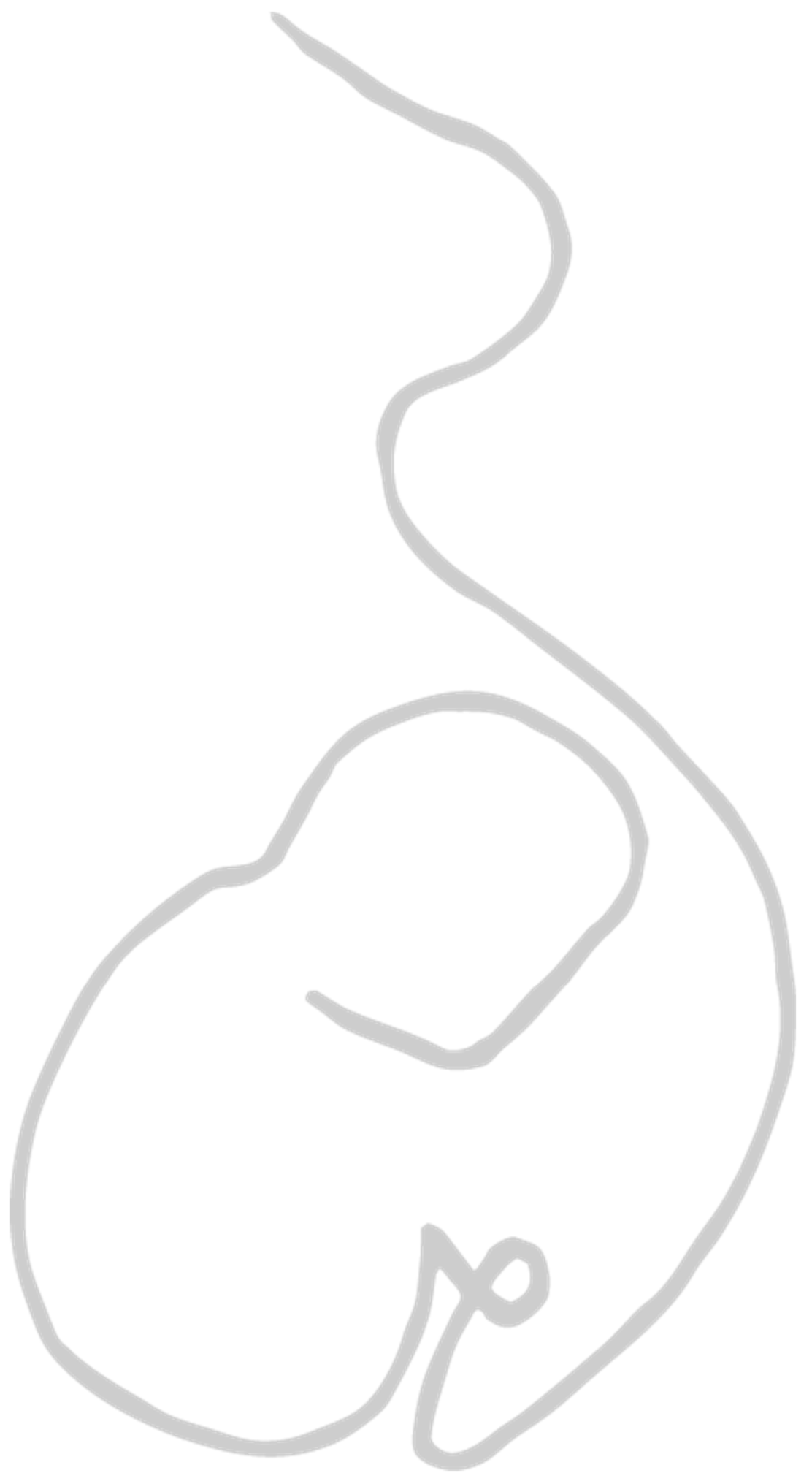




\section{Chapter 3}

\section{Perinatal high-fat diet influences ozone-induced responses on pulmonary oxidant status and the molecular control of mitophagy in female rat offspring}

Sven H. Rouschop, Samantha J. Snow, Urmila P. Kodavanti, Marie-José Drittij, Lou M. Maas, Antoon Opperhuizen, Frederik J. van Schooten, Alexander H. Remels, Roger W.

Godschalk

International journal of molecular sciences, 2021, 22.14: 7551. 


\begin{abstract}
Previous research has shown that a perinatal obesogenic, high-fat diet (HFD) is able to exacerbate ozone-induced adverse effects on lung function, injury, and inflammation in offspring, and it has been suggested that mitochondrial dysfunction is implicated herein. The aim of this study was to investigate whether a perinatal obesogenic HFD affects ozoneinduced changes in offspring pulmonary oxidant status and the molecular control of mitochondrial function. For this purpose, female Long-Evans rats were fed a control diet or HFD before and during gestation, and during lactation, after which the offspring were acutely exposed to filtered air or ozone at a young-adult age (forty days). Directly following this exposure, the offspring lungs were examined for markers related to oxidative stress; oxidative phosphorylation; and mitochondrial fusion, fission, biogenesis, and mitophagy. Acute ozone exposure significantly increased pulmonary oxidant status and upregulated the molecular machinery that controls receptor-mediated mitophagy. In female offspring, a perinatal HFD exacerbated these responses, whereas in male offspring, responses were similar for both diet groups. The expression of the genes and proteins involved in oxidative phosphorylation and mitochondrial biogenesis, fusion, and fission was not affected by ozone exposure or perinatal HFD. These findings suggest that a perinatal HFD influences ozone-induced responses on pulmonary oxidant status and the molecular control of mitophagy in female rat offspring.
\end{abstract}




\section{Introduction}

The prevalence of obesity has nearly doubled since 1980 [2], resulting in $52 \%$ of the European Union's (EU) adult population being overweight and $16 \%$ being obese [3]. Maternal obesity during pregnancy negatively negatively affects the offspring's health, predisposing the offspring to obesity, type 2 diabetes, and cardiovascular diseases [4-7]. Furthermore, prenatal obesity affects lung health, with children from obese mothers being more susceptible to wheezing and developing asthma [8,9]. Similarly, a prenatal obesogenic high-fat diet (HFD) in rats and mice has been shown to induce airway hyperresponsiveness, airway inflammation, and increased susceptibility to respiratory infections $[10,11]$. Because of these adverse pulmonary effects, the offspring from obese mothers may be more susceptible to environmental exposures affecting the health of the lung, such as ozone.

Ozone is a secondary air pollutant that originates from photochemical reactions between precursor pollutants, such as nitrogen oxides $\left(\mathrm{NO}_{\mathrm{x}}\right)$ and volatile organic compounds (VOCs), under the influence of sunlight. Upon inhalation, ozone has various adverse effects on the lungs, such as decreased lung function, increased lung inflammation, airway hyperresponsiveness, and increased pulmonary vascular permeability [12-16]. To protect the population against these pulmonary responses and other harmful effects induced by ozone, a target value of $0.06 \mathrm{ppm}$ was set by the EU for air quality standards. Nevertheless, in $2018,34 \%$ of the EU's urban population was exposed to ozone concentrations sufficiently high to cause harmful pulmonary effects [17].

Despite the increasing obesity prevalence and a large part of the urban population being exposed to harmful ozone levels, little research has been done on the combined effects of prenatal obesity and air pollution on the lungs of offspring. So far, two studies have shown that ozone-induced detrimental effects on lung function, injury, and inflammation in rat offspring were more profound in the offspring from dams that were fed a HFD diet during gestation $[18,19]$. Furthermore, a study deploying a systemic metabolomics approach demonstrated that a perinatal HFD exacerbated ozone-induced effects on lipid, protein, and energy metabolism, which suggested an impairment of mitochondrial function [1]. To elucidate whether mitochondria and changes in the molecular machinery that control the mitochondrial content and function are involved in the effect of maternal obesity on ozone susceptibility in offspring, we performed an experiment in which female rats were fed a HFD before and during gestation, and during lactation, after which the offspring were exposed to ozone at a young-adult age (forty days). Directly following this exposure, the offspring lungs were examined for markers related to mitochondrial content and function, such as oxidative stress; oxidative phosphorylation; and mitochondrial fusion, fission, biogenesis, and mitophagy. In this paper, we show that acute ozone exposure increases oxidative status 
and upregulates the molecular control of receptor-mediated mitophagy in the lungs of young-adult rats, and that these responses are exacerbated in female offspring from dams fed a perinatal HFD.

\section{Materials and methods}

\section{Animals, diet, and breeding}

The animal experiments were approved by the U.S. Environmental Protection Agency's (EPA) Institutional Animal Care and Use Committee (LAPR\#18-06-002). All of the animals were SPF-free, housed under $12 \mathrm{~h} / 12 \mathrm{~h}$ light/dark cycles, and had ad libitum access to food and water. Initial feeding and breeding procedures were performed by Charles River Laboratories (Raleigh, NC, USA). Thirty-day-old female Long-Evans rats were fed either a control diet (CD; 10 kcal\% fat; TD.08806, Harlan Laboratories, Teklad Custom Research Diets, Madison, WI, USA) or a high-fat diet (HFD; $60 \mathrm{kcal} \%$ fat, TD.06414) throughout the experiment (Figure 1). Females were bred on postnatal day (PND) 72 and shipped to the close-by EPA animal facility on gestational day (GD) 1 . From each litter ( $n=10$ per diet group), two pups per sex were randomly selected and allocated to either the control or ozone exposure group (1 pup/sex/litter/exposure; $n=10$ pups per sex/exposure/diet group). The offspring were first weaned onto the maternal diet (CD or HFD) on PND 21, and switched to CD on PND 29.
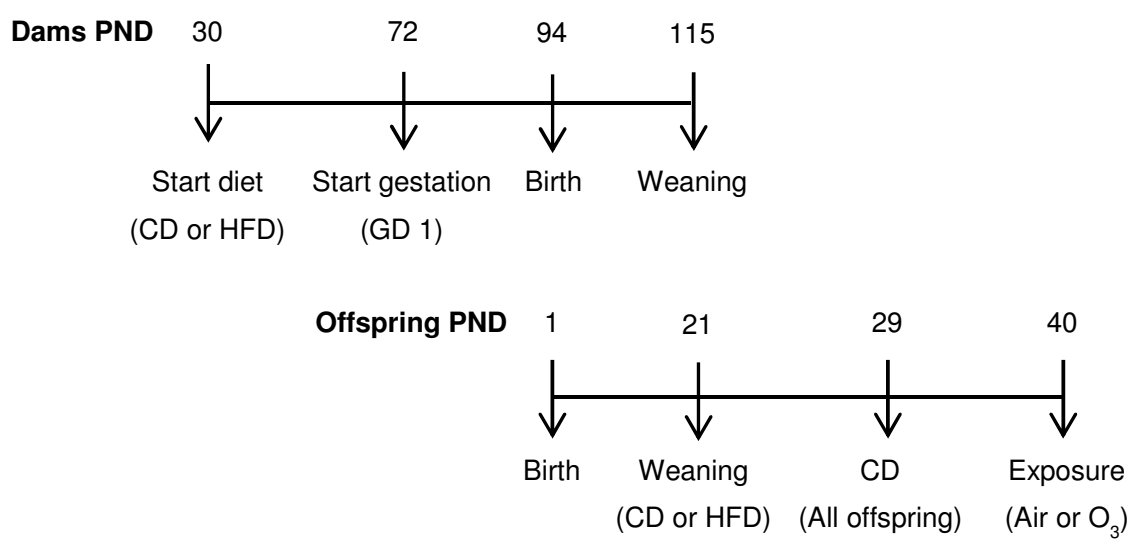

Euthanasia

Figure 7. Experimental design. PND—postnatal day; GD-gestational day; CD-control diet; HFDhigh-fat diet; $\mathrm{O}_{3}-$ ozone. 


\section{Ozone exposure}

On PND 40, the offspring were exposed to either filtered air or ozone ( $0.8 \mathrm{ppm})$ for $5 \mathrm{~h}$. The animals were exposed in Rochester style "Hinners" chambers. Ozone was generated from oxygen using a silent arc discharge generator (OREC, Phoenix, AZ, USA) and was transported to the exposure chambers. All of the exposures occurred between 7 am and 11 am so as to avoid diurnal variation in biological endpoints. The mean ( \pm standard error of the mean (SEM)) air temperature, relative humidity, and ozone concentration in the exposure chambers were $22.47 \pm 0.14{ }^{\circ} \mathrm{C}, 52.72 \pm 0.22 \%$, and $0.0 \pm 0.0 \mathrm{ppm}$, respectively, for filtered air exposure, and $23.24 \pm 0.12{ }^{\circ} \mathrm{C}, 51.09 \pm 0.22 \%$, and $0.7999 \pm 0.0016 \mathrm{ppm}$, respectively, for ozone exposure.

\section{Euthanasia and sample collection}

Within two hours after filtered air or ozone exposure, the animals were euthanized with an overdose of sodium pentobarbital (>200 mg/kg; Fatal Plus, Vortech Pharmaceuticals, LTD., Dearborn, MI, USA). The unlavaged left lung was snap frozen and stored at $-80{ }^{\circ} \mathrm{C}$ until further assessment of the glutathione levels, total antioxidant capacity, lipid peroxidation, gene expression, and protein expression.

\section{Glutathione status}

The glutathione status was assessed by measuring reduced and oxidized glutathione (GSH and GSSG, respectively). To measure GSSG, the samples were first depleted of GSH by incubating the lung homogenates with $3 \%(v / v)$ 2-vinylpyridine (Sigma-Aldrich, Saint Louis, MO, USA) and $6 \%(v / v)$ ethanol for $1 \mathrm{~h}$ at room temperature. To measure the GSH + GSSG, lung homogenates were prepared in $1.3 \%(w / v) 5$-sulfosalicylic acid (Sigma-Aldrich) so as to prevent the oxidation of GSH. Reactions were started by adding $100 \mu \mathrm{L}$ reagent, containing $0.3 \mathrm{mM}$ 5,5-dithiobis(2-nitro-benzoic acid) (Sigma-Aldrich) and $0.4 \mathrm{mM} \mathrm{NADPH}$ (SigmaAlrich), and $50 \mu \mathrm{L}$ glutathione reductase $(4 \mathrm{U} / \mathrm{mL})$ to $50 \mu \mathrm{L}$ prepared lung homogenates. Absorbance was recorded at $412 \mathrm{~nm}$ for $3 \mathrm{~min}$ at $20 \mathrm{~s}$ intervals. GSSG and GSH + GSSG concentrations were determined using the reaction rate and standard curves. The GSH and GSSG concentrations were normalized to the starting amount of lung tissue. The GSSG/GSH ratio was calculated as a measure for the glutathione status.

\section{Total antioxidant capacity}

The total antioxidant capacity was determined using the trolox equivalent antioxidant capacity (TEAC) assay. Lung homogenates were deproteinized by incubating samples in $5 \%$ 
trichloric acid for $5 \mathrm{~min}$ on ice, followed by centrifugation at 15,000 rpm for $5 \mathrm{~min}$. To assess the antioxidant capacity, supernatants were incubated with a radical solution, containing 5 $\mathrm{mM} \mathrm{2,2}$ azino-bis-3 ethylbenzothiazoline-6-sulfonic acid (Sigma-Aldrich), $20 \mu \mathrm{M} \mathrm{H}_{2} \mathrm{O}_{2}$, and $20 \mathrm{mU} / \mathrm{mL}$ horseradish peroxidase (Sigma-Aldrich) at $37^{\circ} \mathrm{C}$ for $5 \mathrm{~min}$, and the absorbance was measured at $734 \mathrm{~nm}$. TEAC was calculated by normalizing the absorbances to an antioxidant capacity of $1 \mu \mathrm{M}$ Trolox, and was further normalized to the starting amount of lung tissue.

\section{Lipid peroxidation}

The lipid peroxidation was assessed by measuring the malondialdehyde (MDA) levels using the thiobarbituric acid reactive substances (TBARS) assay. Lung homogenates were incubated with a reagent containing $12 \mathrm{mM}$ 2-thiobarbituric acid (Merck-Millipore, Burlington, MA, USA), 0.01\% ( $w / v$ ) EDTA (Merck-Millipore), $320 \mathrm{mM} \mathrm{H}_{3} \mathrm{PO}_{4}$, and $6.81 \mathrm{mM}$ butylated hydroxytoluene (Sigma-Aldrich) at $100{ }^{\circ} \mathrm{C}$ for $1 \mathrm{~h}$. The reaction was stopped by putting samples on ice, $0.5 \mathrm{vol}$. 1-butanol was added, and the samples were vortexed and centrifuged at $12,000 \mathrm{rpm}$ for $3 \mathrm{~min}$. Fluorescence was measured in supernatant at $\lambda_{\text {exc. }}=$ $530 \mathrm{~nm}$ and $\lambda_{\mathrm{em}} .=560 \mathrm{~nm}$. The MDA levels were quantified using a standard curve and were normalized to the starting amount of lung tissue.

\section{Gene expression}

The total RNA was extracted from the offspring lung using TRIzol Reagent (Thermo Fischer Scientific, Waltham, MA, USA). Then, 400 ng RNA was reverse transcribed into the cDNA with iScript (Bio-Rad, Hercules, CA, USA). Real-time qPCR was performed with $4.4 \mu \mathrm{L}$ of 1:50 diluted CDNA, target-specific primers (Table S1), SensiMix SYBR and Fluorescein Kit (Meridian Bioscience, Cincinnati, OH, USA), and a LightCycler 480 (Roche, Basel, Switzerland). The gene expression levels were quantified with LinRegPCR software version 2014.0 [20] and normalized using the geometric mean of the expression levels of the reference genes Tuba1b, Rpl13a, and Ppia. Samples with non-specific melt curves or suboptimal amplification (detected as unstable baseline or no plateau in the amplification curve by LinRegPCR software) were excluded from the analysis, leading to $n=9-10$ per group for all of the examined genes.

\section{Protein expression}

The offspring lung lysates were prepared in an immunoprecipitation buffer (Table S2). The protein concentrations of the lysates were measured with Pierce BCA Protein Assay Kit (Thermo Fischer Scientific, Waltham, MA, USA). Lysates were boiled in a 1× Laemmli buffer 
(Table S3) and $10 \mu \mathrm{g}$ protein per sample was separated on $12 \%$ Criterion XT Bis-Tris Protein Gel (Bio-Rad) and blotted onto $0.45 \mu \mathrm{m}$ nitrocellulose membranes (Bio-Rad). The membranes were stained for the total protein with PonceauS (Sigma-Aldrich, Saint Louis, MO, USA) and imaged on the Amersham Imager 600 (GE Healthcare, Chicago, IL, USA). For the antigen-specific expression levels, the membranes were probed with primary antibodies (Table S4) and HRP-conjugated secondary antibodies (Table S5), incubated with SuperSignal West PICO or Femto Chemiluminescent Substrate (Thermo Fisher Scientific), and imaged on the Amersham Imager 600. The total protein levels and antigen-specific expression were quantified with ImageJ version 1.53e [21]. The antigen-specific expression was normalized for the total protein levels. Given the large number of animals, the samples were analyzed on four different gels/blots. To prevent bias from this, an equal number of samples from each experimental group was analyzed on each gel/blot. In addition, between-gel/blot variation was removed using factor correction [22], which was confirmed using principle component analysis. For some offspring animals, insufficient tissue was left over for the protein analysis after all of the previous measurements, leading to $n=7$ for ozone-exposed CD females and $n=9-10$ for all of the other experimental groups.

\section{Statistical analysis}

All data were stratified for sex and analyzed using two-way ANOVA, with diet and exposure as factors. First, it was checked whether both factors had a significant interaction. If not, the interaction term was removed from the analysis. Next, it was tested whether one or both factors had a significant effect on the outcome. For those factors with a significant effect, Holm-Sidak post-hoc test was used to compare the means. In addition to these primary analyses, associations were assessed between the glutathione status, total antioxidant capacity, lipid peroxidation, gene expression, and protein expression vs. previously published outcomes from the same experiment, such as markers for lung injury and inflammation [1]. These associations were determined using Pearson's productmoment correlation after stratification for sex and diet. Given the large number of correlations that were tested, $p$-values were corrected for multiple testing by computing the false discovery rate q-values using the Benjamini-Hochberg procedure [23]. The results were graphically presented as boxplots, with boxes representing the first quartile, median, and third quartile, and whiskers representing the minimum and maximum values within 1.5 times the interquartile range (IQR) below the first quartile or above the third quartile, respectively, and with points representing the values outside the limits of the whiskers. $p<$ 0.05 or $q<0.05$ was considered significant. All of the statistical analyses were performed in $R$ version 4.0.2 (R Foundation for Statistical Computing, Vienna, Austria). 

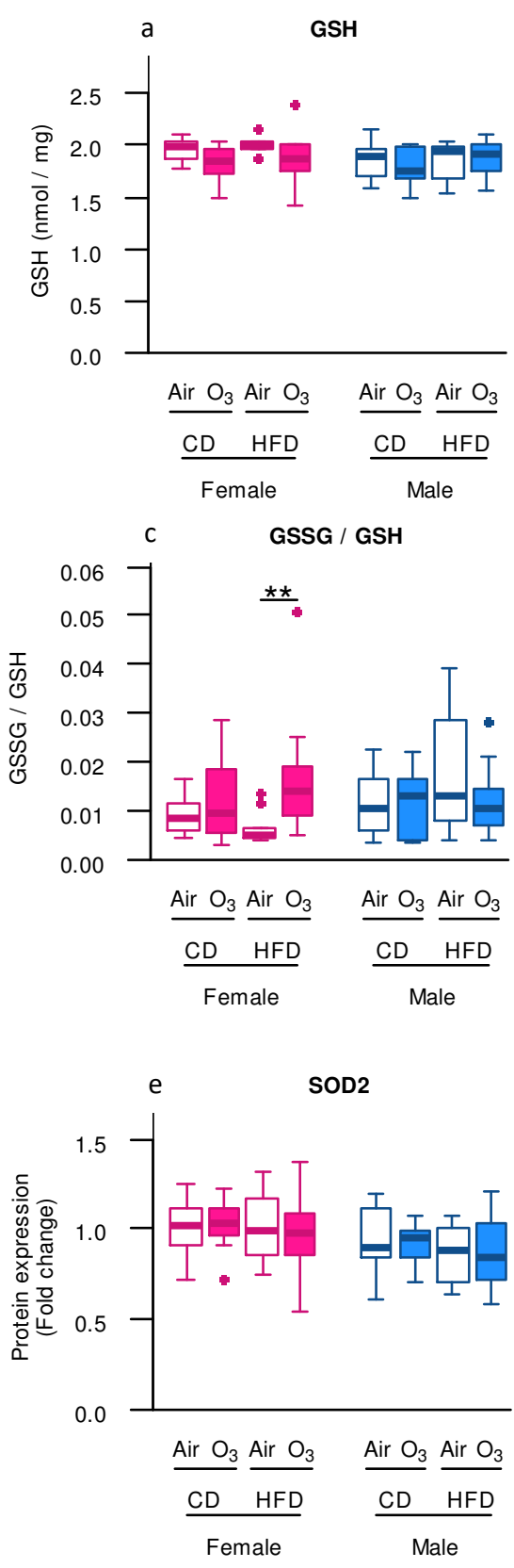
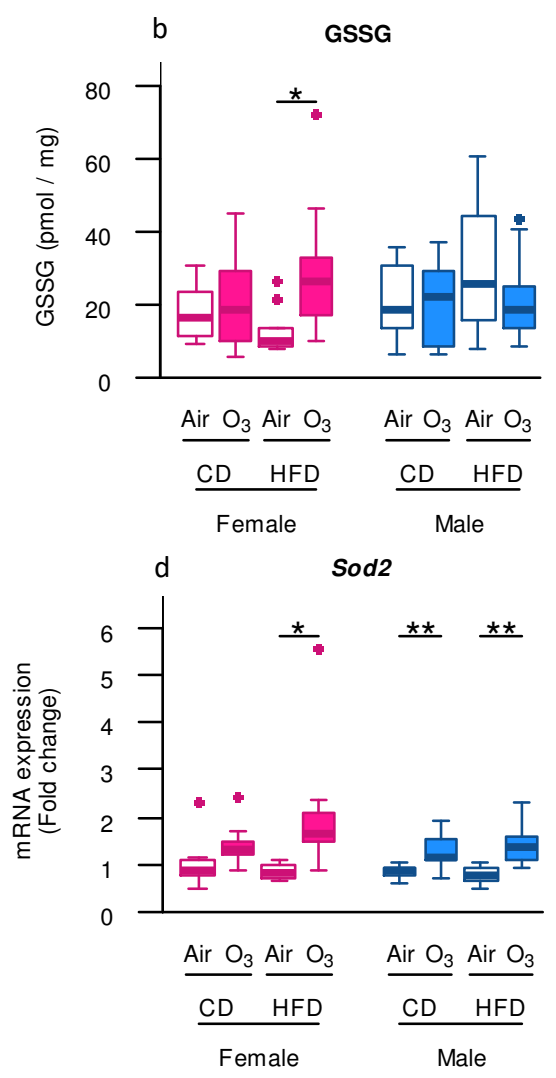

Figure 2. Acute ozone exposure increased pulmonary oxidant status in female offspring from high-fat diet (HFD) dams and all male offspring. (a) Reduced glutathione (GSH), (b) oxidized glutathione (GSSG) and (c) GSSG/GSH ratio, (d) Sod 2 mRNA expression, and (e) SOD2 protein expression were assessed within two hours after exposure to filtered air or ozone in the lungs of female and male offspring from dams fed a perinatal control diet (CD) or HFD. Data are presented as boxplots. Statistical analysis by two-way ANOVA with a Holm-Sidak post-hoc test. $n=10$ per group (a-c), $n=9-10$ per group (d), or $n=7-10$ per group (e). $* p<$ $0.05, * * p<0.01 . \mathrm{O}_{3}$-ozone. 


\section{Results}

\section{Acute ozone exposure increases pulmonary oxidant status}

We previously showed that the perinatal HFD compared with the CD increased the body weight and fat percentage of dams throughout gestation, as well as of the offspring at postnatal day (PND) 37 [1,19]. Furthermore, acute ozone exposure was shown to cause pulmonary protein leakage and lung cell injury in both female and male offspring, and induced neutrophilic inflammation in female offspring [1]. We next studied whether these ozone-induced pulmonary responses in offspring were associated with alterations in oxidative stress markers and whether these responses were affected by maternal diet. For the antioxidant glutathione, reduced (GSH) levels were not affected (Figure 2a), but ozone exposure did significantly increase the levels of oxidized glutathione (GSSG), and therefore the GSSG/GSH ratio, compared with air exposure in female offspring from HFD dams (Figure $2 b, c)$. Ozone exposure also significantly upregulated mRNA expression of the mitochondrial antioxidant superoxide dismutase 2 (Sod2) in female offspring from HFD dams, and male offspring from both CD and HFD dams (Figure 2d). In addition, Sod2 mRNA levels correlated significantly with previously published [1] pulmonary vascular leakage markers albumin and total protein in broncheoalveolar lavage fluid (BALF) in female and male offspring from HFD dams (Table 1). Altogether, these data suggest that acute ozone exposure increased the pulmonary oxidant status in female offspring from HFD dams and all male offspring.

Table 1. Lung Sod2 mRNA levels correlated significantly with broncheoalveolar lavage fluid (BALF) vascular leakage markers albumin and total protein in female and male offspring from high-fat diet (HFD) dams. All of the markers were assessed within two hours after exposure to filtered air or ozone in the lungs or BALF from female and male offspring from dams fed a perinatal control diet (CD) or HFD. BALF albumin and total protein data were previously published [1]. Only significant correlations $(q<0.05)$ are shown in this table. Statistical analysis by Pearson's product-moment correlation after stratification for sex and diet. False discovery rate q-values were computed from $p$-values using the Benjamini-Hochberg procedure to correct for multiple testing.

\begin{tabular}{cccc}
\hline Correlation & Stratum & r & q-Value \\
\hline & Female CD & 0.42 & 0.624 \\
Sod2 mRNA vs. BALF albumin & Female HFD & 0.78 & 0.014 \\
& Male CD & 0.59 & 0.462 \\
& Male HFD & 0.82 & 0.003 \\
\hline \multirow{3}{*}{ Sod2 mRNA vs. BALF total protein } & Female CD & 0.49 & 0.550 \\
& Female HFD & 0.77 & 0.014 \\
& Male CD & 0.58 & 0.462 \\
& Male HFD & 0.80 & 0.004 \\
\hline
\end{tabular}


Not all oxidative stress markers, however, were affected by ozone exposure. Although the Sod 2 mRNA levels were upregulated in most offspring groups, this did not lead to increased SOD2 protein levels in any of the offspring groups (Figure 2e). Furthermore, no differences were observed in the Trolox equivalent antioxidant capacity (TEAC) and levels of the lipid peroxidation product malondialdehyde (MDA) (Figure 3a,b), indicating that acute ozone exposure and perinatal HFD did not alter the total antioxidant capacity or induce oxidative damage to lipids, respectively, in the offspring lungs. The mRNA expression of cytoplasmic antioxidants catalase (Cat) and superoxide dismutase 1 (Sod1) were also not altered by ozone exposure or perinatal HFD (Figure 3c,d).
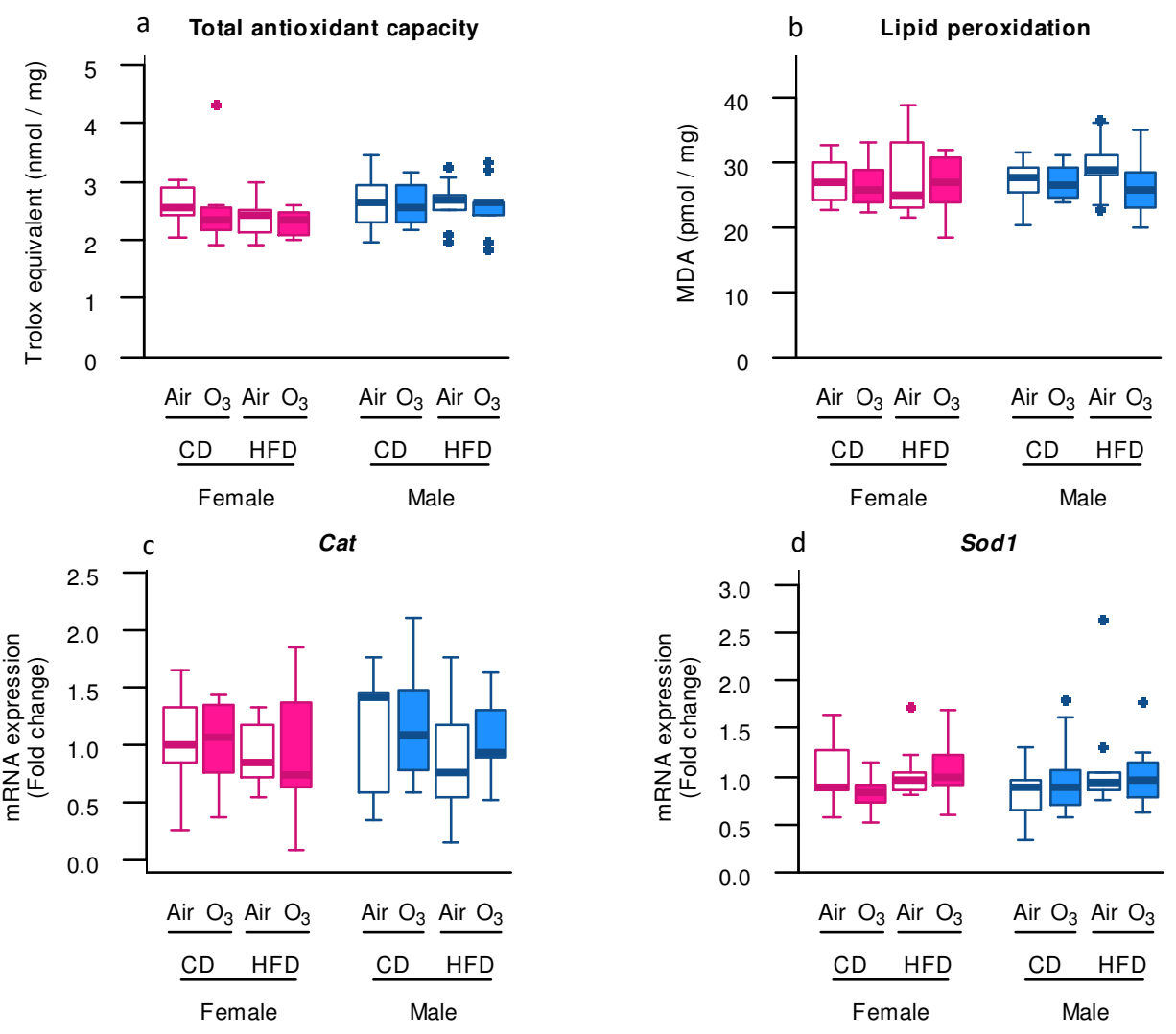

Figure 3. Acute ozone exposure and perinatal high-fat diet (HFD) did not alter the pulmonary (a) total antioxidant capacity, (b) lipid peroxidation, or expression of cytoplasmic antioxidant genes (c) Cat and (d) Sod1. All of the markers were assessed within two hours after exposure to filtered air or ozone in the lungs of female and male offspring from dams fed a perinatal control diet (CD) or HFD. Data are presented as boxplots. Statistical analysis by two-way ANOVA with a Holm-Sidak post-hoc test. $n=10$ per group $(\mathrm{a}, \mathrm{b})$ or $n=9-10$ group $(\mathrm{c}, \mathrm{d})$. $\mathrm{O}_{3}$-ozone. 

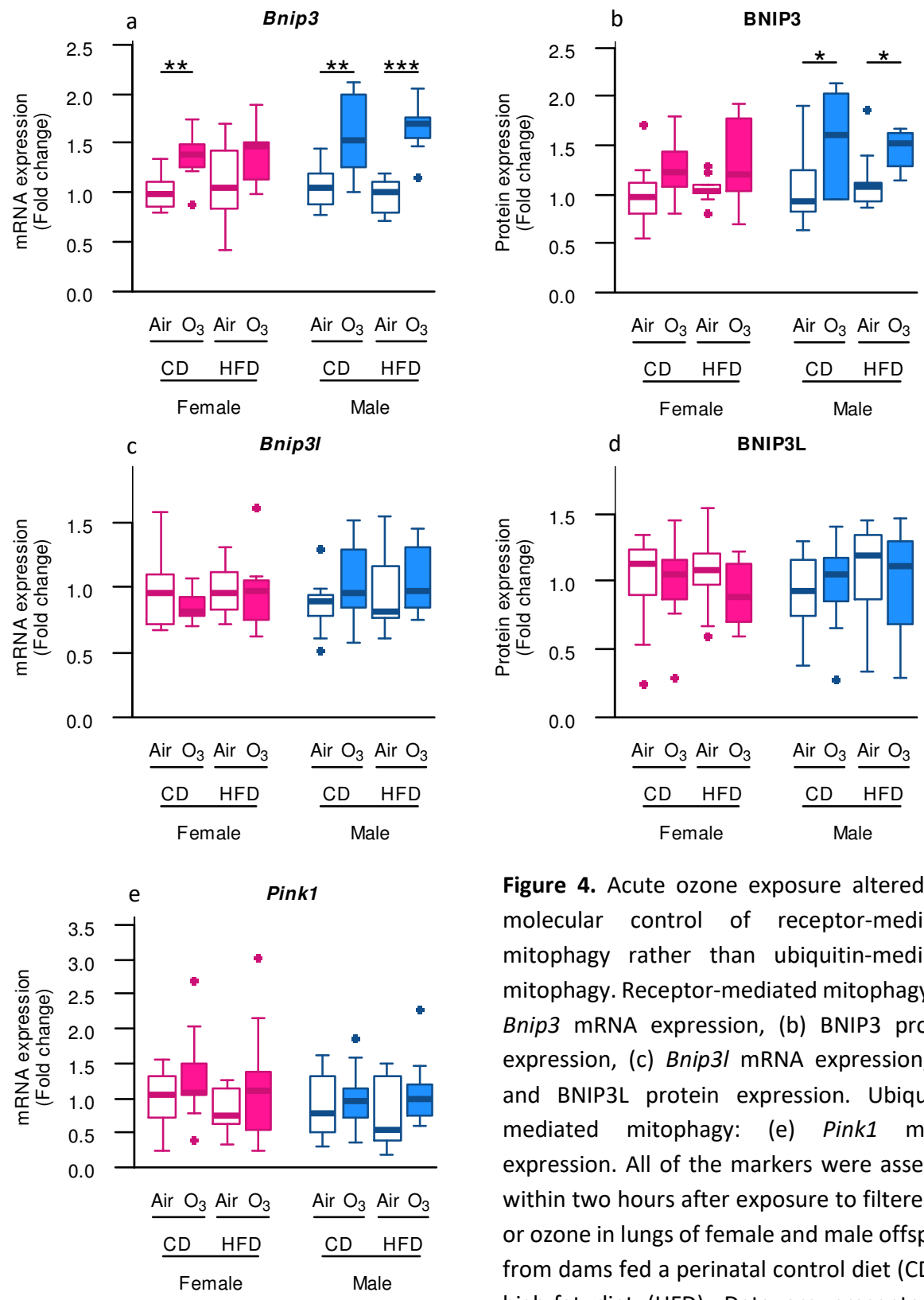

Figure 4. Acute ozone exposure altered the molecular control of receptor-mediated mitophagy rather than ubiquitin-mediated mitophagy. Receptor-mediated mitophagy: (a) Bnip3 mRNA expression, (b) BNIP3 protein expression, (c) Bnip3/ mRNA expression, (d) and BNIP3L protein expression. Ubiquitinmediated mitophagy: (e) Pink1 mRNA expression. All of the markers were assessed within two hours after exposure to filtered air or ozone in lungs of female and male offspring from dams fed a perinatal control diet (CD) or high-fat diet (HFD). Data are presented as boxplots. Statistical analysis by two-way ANOVA with a Holm-Sidak post-hoc test. $n=$ 9-10 per group $(\mathrm{a}, \mathrm{c}, \mathrm{e})$ or $n=7-10$ per group (b,d). ${ }^{*} p<0.05, * * p<0.01,{ }^{* * *} p<0.001$. $\mathrm{O}_{3}$-ozone. 
Acute ozone exposure alters the abundance of key constituents involved in receptormediated mitophagy

As acute ozone exposure upregulated the mRNA expression of the mitochondrial antioxidant Sod2, but not of the cytoplasmic antioxidants Cat and Sod1, we next examined whether the molecular control of the mitochondrial content in the offspring lungs was affected. Considering that mitochondria are not only a major source of reactive oxygen species (ROS), but can also be damaged by it, we first assessed the gene and protein expression of key regulators of mitophagy. As a result, the mRNA expression of B-cell lymphoma 2/adenovirus E1B $19 \mathrm{kDa}$ protein-interacting protein 3 (Bnip3), a mitophagy receptor [24], was significantly upregulated after ozone exposure in female offspring from $\mathrm{CD}$ dams and all male offspring (Figure 4a). Similarly, the BNIP3 protein expression was significantly increased after ozone exposure in the male offspring from CD and HFD dams (Figure 4b). The mRNA and protein expression of B-cell lymphoma 2/adenovirus E1B $19 \mathrm{kDa}$ protein-interacting protein 3-like (Bnip3I), a mitophagy receptor with a close homology to Bnip3 [25], were not affected by ozone exposure or perinatal HFD (Figure 4c,d). Likewise, no differences were observed in the mRNA expression of phosphatase and tensin homologinduced kinase 1 (Pink1; Figure 4e), a gene essential for ubiquitin-mediated mitophagy [26]. These results suggest that acute ozone exposure induced changes in the abundance of the molecular machinery controlling the receptor-mediated mitophagy in the offspring lungs rather than ubiquitin-mediated mitophagy in the female offspring from CD dams and all male offspring.

\section{Acute ozone exposure and perinatal HFD do not alter the expression of the oxidative phosphorylation machinery}

As ozone exposure seemed to alter the molecular regulation of mitophagy, we further assessed the abundance of key constituents of the complexes involved in oxidative phosphorylation. However, no differences were observed in the mRNA and protein levels of subunits of oxidative phosphorylation complexes I to V (Figure $5 \mathrm{a}-\mathrm{g}$ ), demonstrating that acute ozone exposure or perinatal HFD did not affect the expression of the oxidative phosphorylation machinery in the offspring lungs. 

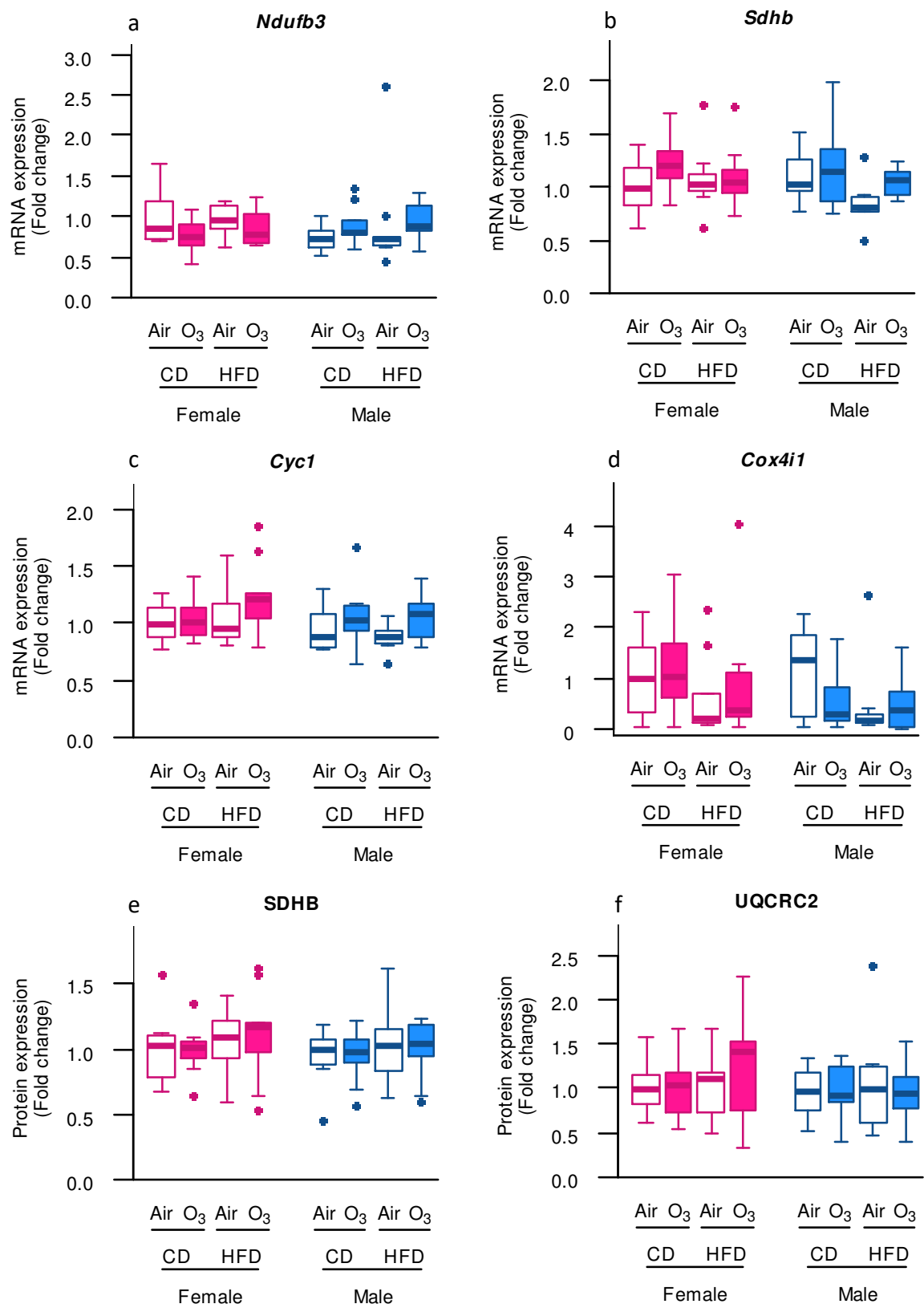

Figure 5 (continued on next page). 


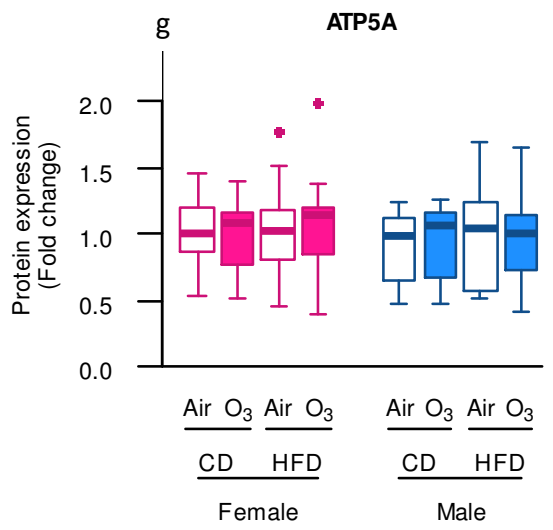

Figure 5. Perinatal high-fat diet (HFD) or acute ozone exposure did not change the pulmonary expression of oxidative phosphorylation machinery subunits. mRNA expression of (a) Ndufb3 (complex I), (b) Sdhb (complex II), (c) Cyc1 (complex III), and (d) Cox4i1 (complex IV), and the protein expression of (e) SDHB (complex II), (f) UQCR2 (complex III), and (g) ATP5A (complex V) were assessed within two hours after exposure to filtered air or ozone in the lungs of female and male offspring from dams fed a perinatal control diet (CD) or HFD. Data are presented as boxplots. Statistical analysis by two-way ANOVA with Holm-Sidak post-hoc test. $n=9-10$ per group $(\mathrm{a}-\mathrm{d})$ or $n=$ 7-10 per group (e-g). $\mathrm{O}_{3}$-ozone.
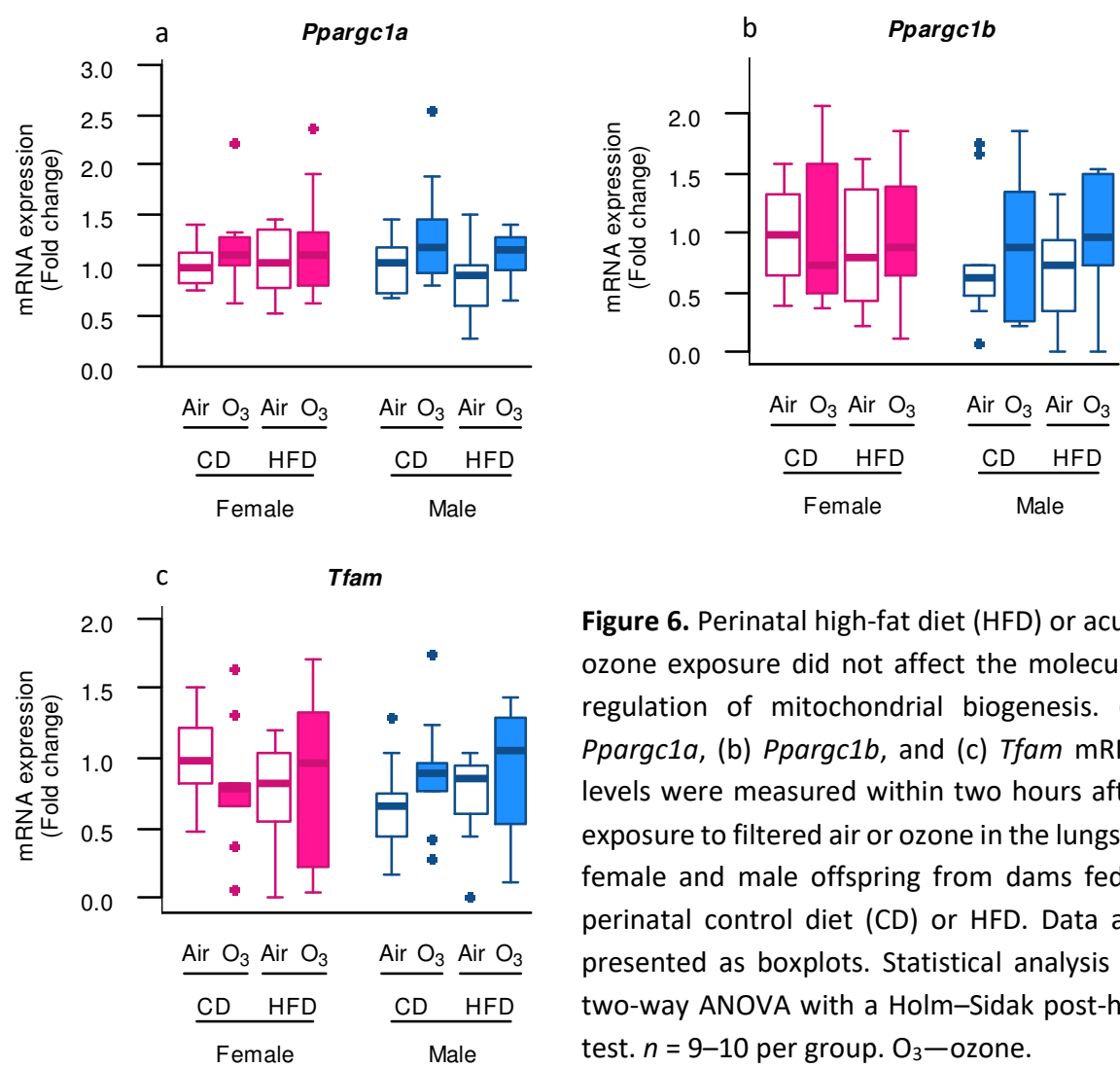

Figure 6. Perinatal high-fat diet (HFD) or acute ozone exposure did not affect the molecular regulation of mitochondrial biogenesis. (a) Ppargc1a, (b) Ppargc1b, and (c) Tfam mRNA levels were measured within two hours after exposure to filtered air or ozone in the lungs of female and male offspring from dams fed a perinatal control diet (CD) or HFD. Data are presented as boxplots. Statistical analysis by two-way ANOVA with a Holm-Sidak post-hoc test. $n=9-10$ per group. $\mathrm{O}_{3}-$ ozone. 

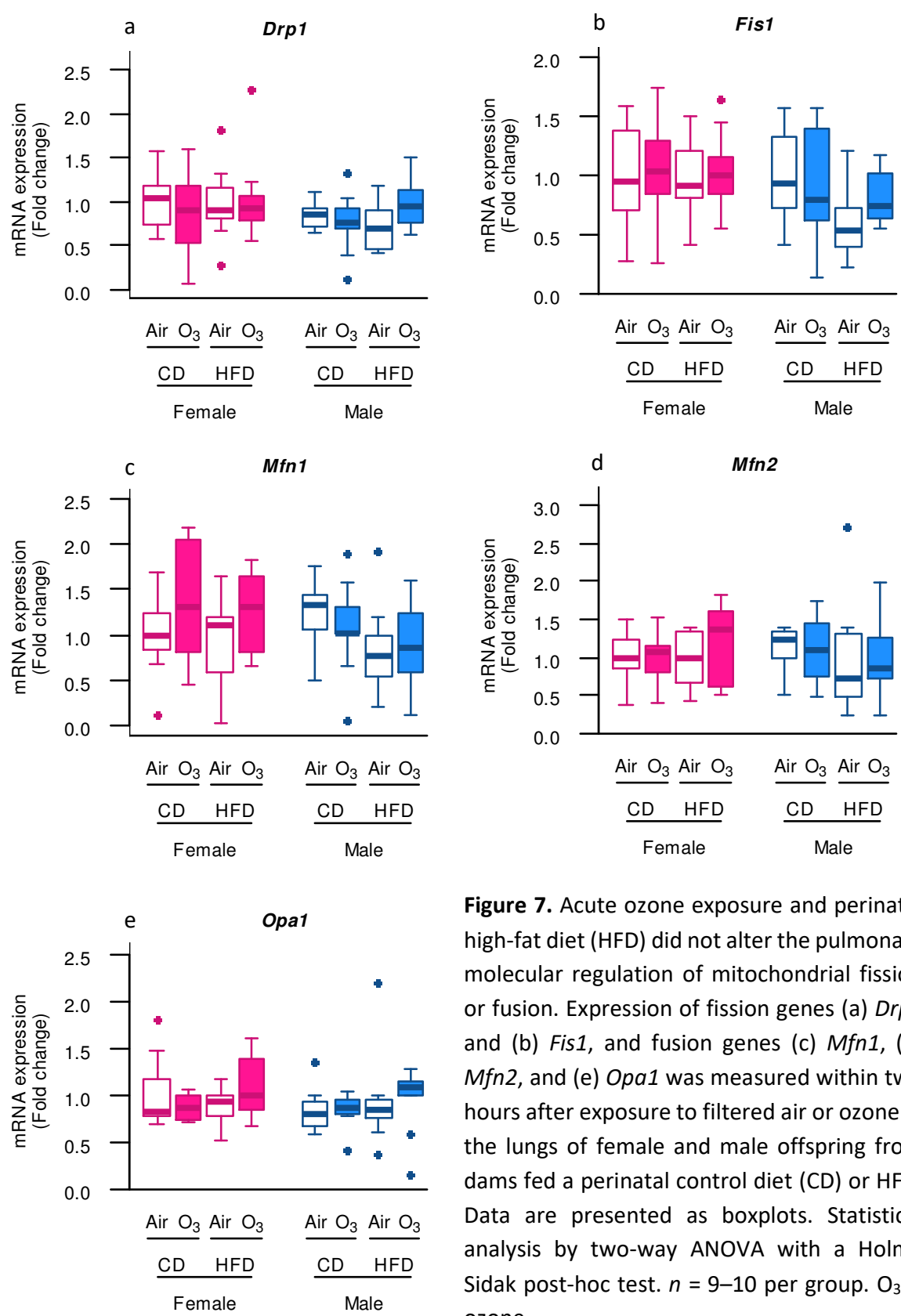

Figure 7. Acute ozone exposure and perinatal high-fat diet (HFD) did not alter the pulmonary molecular regulation of mitochondrial fission or fusion. Expression of fission genes (a) Drp1 and (b) Fis1, and fusion genes (c) Mfn1, (d) $M f n 2$, and (e) Opa1 was measured within two hours after exposure to filtered air or ozone in the lungs of female and male offspring from dams fed a perinatal control diet (CD) or HFD. Data are presented as boxplots. Statistical analysis by two-way ANOVA with a HolmSidak post-hoc test. $n=9-10$ per group. $\mathrm{O}_{3}-$ ozone. 


\section{Acute ozone exposure and perinatal HFD do not affect the abundance of key regulators of mitochondrial biogenesis, fission or fusion in lung tissue}

To further test whether the molecular control of the mitochondrial content was affected, we measured the expression of the master regulators of mitochondrial biogenesis peroxisome proliferator-activated receptor gamma coactivator 1-alpha (Ppargc1a) and peroxisome proliferator-activated receptor gamma coactivator 1-beta (Ppargc1b), and of a key regulator of mitochondrial gene expression transcription factor a, mitochondrial (Tfam). However, the mRNA expression of these genes was not altered (Figure 6), indicating that mitochondrial biogenesis was not affected by acute ozone exposure or perinatal HFD. Next to the mitophagy and biogenesis, mitochondrial function and content is also regulated through the events of fission and fusion. To examine whether mitochondrial fission and fusion were altered by ozone exposure or perinatal HFD, the gene expression of fission mediators dynamin-related protein 1 (Drp1) and mitochondrial fission protein 1 (Fis1) was examined, as well as the gene expression of fusion mediators mitofusin-1 (Mfn1), mitofusin2 (Mfn2), and optic atrophy 1 (Opa1). No differences were observed, however, in the mRNA levels of any of these genes (Figure 7a-e). Altogether, these data suggest that the processes of mitochondrial biogenesis, fission, and fusion were likely not affected in the offspring lungs by acute ozone exposure or perinatal HFD.

\section{Discussion}

Previous research has shown that a perinatal HFD is able to exacerbate ozone-induced pulmonary responses, and has suggested mitochondrial dysfunction to be involved in this effect $[1,18,19]$. Whether or not disturbances in the molecular regulation of the mitochondrial content and function are associated with this, however, is unknown. We therefore examined the lungs of offspring exposed to a perinatal HFD and ozone for molecular markers related to oxidative stress and the regulation of the mitochondrial content. We showed that acute ozone exposure increased pulmonary oxidant status and upregulated the abundance of the key constituents involved in the molecular control of receptor-mediated mitophagy. For female offspring, perinatal HFD aggravated these ozone responses, whereas for male offspring, the responses were similar in both diet groups. Abundances of mRNAs and proteins involved in oxidative phosphorylation and mitochondrial biogenesis, fusion, and fission were not affected by ozone exposure or perinatal HFD.

First, we showed that acute ozone exposure upregulated the mRNA expression of the antioxidant gene Sod 2 in all offspring, except for the females from CD dams. In addition, the 
glutathione oxidation status (GSSG/GSH ratio) was increased in female offspring from HFD females. Collectively, these results suggest that ozone exposure increased the pulmonary oxidant status, and that perinatal HFD aggravated these responses in female offspring only. The upregulation of Sod 2 in response to both acute and chronic ozone exposure had previously been shown in other animal studies [27,28]. In addition, both Sod2 and glutathione appear to play an important role in protecting the mitochondria against ozoneinduced oxidative stress. For instance, Sod2 overexpression and glutathione supplementation reduced p62 knockout-induced mitochondrial ROS in mouse embryonic fibroblasts [29]. Furthermore, increasing the intracellular GSH pool in yeast was shown to reduce fasting-induced mitophagy [30,31], but not other non-selective forms of autophagy [30]. In addition, genetic linkage analyses have linked the Sod 2 and glutathione peroxidase $(G p \times 1)$ genes to differential ozone susceptibility in mice [32,33]. Next to these specific effects on Sod2 and glutathione, ozone exposure has also previously been shown to induce pulmonary oxidative stress in general. In both acute and chronic exposure studies in mice, ozone exposure induced pulmonary oxidative stress [34-36]. The administration of MitoQ or MitoTEMPO, which are a mitochondrion-specific antioxidant and an inhibitor of mitochondrial ROS, respectively, reversed not only the ozone-induced oxidative stress, but also restored other ozone-induced pulmonary responses, such as lung inflammation and airway hyperresponsiveness [34-36], suggesting that oxidative stress is an important mediator of other, more phenotypical pulmonary ozone responses. This may explain the correlations we found between the Sod 2 mRNA levels and BALF albumin and total protein levels, which are both markers for lung damage.

In contrast with Sod2 expression, acute ozone exposure and perinatal HFD did not affect the mRNA expression of Cat and Sod1. Similar to Sod2, Sod1 is responsible for converting superoxide to hydrogen peroxide and oxygen, whereas Cat catalyzes the decomposition of hydrogen peroxide into water and oxygen. Cat and Sod1 exert their functions mainly in the cytoplasm and peroxisomes, respectively, while Sod 2 is located in the mitochondrial matrix. Given the different effects of ozone and perinatal HFD on expression of Sod2 vs Sod1 and Cat, and taking into account the different localizations of these enzymes, our results may suggest that the oxidative response to ozone exposure was focused at the mitochondria rather than other cellular locations.

The MDA levels were not affected by perinatal HFD or ozone exposure, indicating that neither of these treatments induced oxidative damage to lipids in the offspring lungs. The absence of lipid peroxidation could imply that cells were sufficiently protected by cellular antioxidant systems against the ozone-induced increase in oxidant status. Alternatively, oxidative damage may have occurred at a later time point after ozone exposure, as was previously observed in other studies. Xu et al., for instance, reported elevated MDA levels 
in BALF after acute ozone exposure in mice [36]. However this outcome was assessed $24 \mathrm{~h}$ after exposure, providing the oxidative damage a larger period of time to develop than the two hours used in our study. In addition, $\mathrm{Xu}$ et al. exposed the animals to an ozone concentration of $2.5 \mathrm{ppm}$, which is three times higher than our $0.8 \mathrm{ppm}$. In a study by Li et al., six weeks of ozone exposure did not change the MDA levels in BALF [28]. However, after another six weeks, during which ozone exposure ceased, the MDA levels were increased, indicating a delayed oxidant response. Possibly, lipid peroxidation would have been increased in our study as well, if the pulmonary MDA levels were assessed at a later time point.

In our study, acute ozone exposure increased Bnip3 mRNA expression in all offspring except the females from HFD dams, and increased BNIP3 protein expression in all male offspring. Although many studies have examined the effects of ozone exposure on mitochondrial function and content in general, the current study is the first report, to our knowledge, on the ozone effects on specific mitophagy-related markers. The accumulation of mitophagy receptors such as BNIP3 on the mitochondrial outer membrane initiate mitophagy by promoting the binding of the mitochondrial membrane to the autophagosomal membrane [37]. In light of this, the increased abundance of Bnip3 in our study may have led to a rise in mitophagy as well. Mitophagy aids in maintaining mitochondrial quality [38] and plays an important role in protecting tissues against many forms of injury, such as alcoholic liver disease [39], ischemia/reperfusion-induced acute kidney injury [40], and tumorigenesis [41]. The upregulation of the molecular control of mitophagy in our study may thus be interpreted as a beneficial response, potentially protecting the lung cells against ozoneinduced injury. Female offspring from HFD dams were the only offspring to not upregulate Bnip3 in response to ozone, thereby potentially lacking the protection that an upregulation of mitophagy would offer. This lack of protection against ozone-induced oxidative stress is indeed implied by the increased oxidative status in female offspring from HFD dams, as indicated by the elevated GSSG/GSH ratio and Sod 2 expression. These results suggest that female offspring from dams fed an HFD experienced more detrimental effects from ozone exposure than the other offspring groups. This is in accordance with our previous study [1], which found ozone-induced changes in the gut microbiome metabolites and metabolites indicative of mitochondrial stress in tissues to be more profound in these same female offspring from HFD dams than other offspring groups. For males, the ozone effects did not differ between the offspring from CD or HFD dams. Altogether, these findings suggest that a perinatal HFD influences ozone-induced responses in females, but not in males. The mechanism underlying this sex-specific effect may involve gonadal hormones, as previously suggested by the findings from an ozone exposure study using gonadectomized animals [42]. In this experiment, acute ozone exposure elicited greater detrimental effects in shamoperated females than in males. Moreover, lowering gonadal hormone levels by removing 
the ovaries and testes of females and males, respectively, attenuated ozone-induced effects in females, and aggravated ozone responses in males, indicating the association between sex hormones and ozone susceptibility.

Given the upregulation of the molecular control of mitophagy in our study, we hypothesized that abundance of key constituents and regulators of oxidative phosphorylation, biogenesis, fusion, and fission may also be altered. However, the expression of none of the assessed markers was affected by the perinatal HFD or acute ozone exposure. This finding is in contrast with other studies examining the effect of ozone on mitochondria. In a chronic exposure study in mice, ozone exposure downregulated the pulmonary expression of the gene pathways involved in mitochondrial function and decreased the ATP content; oxidative phosphorylation complex I activity; and oxidative phosphorylation complexes I, III, and V protein levels [35]. In a similar study, chronic ozone exposure in mice increased the pulmonary protein levels of DRP1 and oxidative phosphorylation complexes II and IV [34]. Lastly, in an acute exposure study in mice, ozone exposure increased the abundance of MFN2 and oxidative phosphorylation complexes II and IV [36]. Discrepancies between these studies and our study regarding the effect of ozone on mitochondrial markers may possibly be due to differences in the exposure model (chronic vs. acute) or differences in ozone concentrations ( $\geq 2.5 \mathrm{ppm}$ in other studies vs. $0.8 \mathrm{ppm}$ in our study). In addition, the previously mentioned studies assessed the outcome parameters $24 \mathrm{~h}$ after exposure, whereas we measured them within two hours after exposure, which may not have been enough time for the molecular responses to be detectable.

Although the perinatal HFD influenced the molecular responses to ozone, the perinatal HFD on its own (i.e., without the additional ozone exposure) did not have significant effects on the pulmonary oxidant status or molecular regulation of the mitochondrial content. However, maternal obesity has been demonstrated to have negative effects on oxidative stress and mitochondria in other tissues. Saben et al. found that maternal diet-induced metabolic syndrome increased lipid peroxidation and impaired mitochondrial morphology, dynamics, and metabolism in offspring muscle tissue and oocytes [43]. Similarly, Bruce et al. reported a perinatal HFD increased the expression of genes related to oxidative stress and decreased the activity of all electron transport chain complexes in offspring livers [44]. Lastly, in a study by Larsen et al., a prenatal HFD altered the mitochondrial morphology and impaired mitochondrial dynamism in offspring primary cardiomyocytes, with reductions in both fusion and fission events [45]. It should be noted, however, that none of these studies assessed their outcomes in lung tissue, and we were not able find any studies that did, which may partly explain the difference in findings between our and previous studies. 
In the current study, the offspring animals were exposed to ozone concentrations of 0.8 ppm, which is higher than the ozone levels in controlled human intervention studies. It should be considered, however, that animals were exposed during the day, when rats are physically inactive. Humans, in contrast, either during real-life outdoor exposure or in controlled clinical studies, are generally exposed to ozone during physically active periods. Because of this difference, an ozone exposure of $0.8-1.0 \mathrm{ppm}$ in rats is comparable to an exposure of 0.2-0.4 ppm in humans [46].

The ozone exposure in this study took place when the offspring animals were at an age of forty days, which is comparable to a peri-adolescent age in humans. Around this age of forty days, gonadal hormone concentrations rise in rat, with increases of progesterone and estradiol in females and testosterone in males [47]. As these sex hormones have been associated with ozone susceptibility, the sex differences observed in current study may have been different if the offspring animals would have been exposed and assessed at a different age. Nevertheless, exposing rats at an age of forty days is highly relevant, as children at this peri-adolescent age spend a relatively large part of their time outdoors, resulting in the highest real-life ozone exposure. In addition, children and teens, compared with other populations, are especially vulnerable to the effects of breathing ozone [48].

In contrast to what we hypothesized and found in the literature, many of the molecular markers that we measured in this study were not affected by ozone exposure. As already mentioned in this discussion, this may partly be due to the short amount of time between ozone exposure and tissue collection (two hours), potentially providing an insufficient time for molecular responses to develop. It is, in fact, known that although ozone exposure induces lung injury and inflammation immediately after exposure, the peak in these responses is observed the next day $[49,50]$. It may thus be better for future studies to assess molecular outcomes the day after exposure. Another limitation of our study is that mitochondrial outcomes were assessed only on the level of mRNA and protein expression. Although these outcomes provide valuable insights into the molecular responses to perinatal HFD and ozone exposure, it remains unclear whether they represent changes in mitochondrial function as well. Future studies may address this limitation by additionally assessing functional mitochondrial outcomes, such as the mitochondrial copy number, oxidative phosphorylation enzyme activity, and live imaging of fusion and fission events. In addition, future studies may also expand their focus to other relevant organs, such as the liver, given the involvement of oxidative stress and mitochondria in various liver conditions [51,52], and the adverse effects both perinatal HFD and ozone exposure are known to have on the liver $[40,49,50]$. Next to this, future studies may use a chronic exposure model instead of the acute exposure used in current study. Combining a perinatal HFD with such chronic ozone exposure may very well lead to different results than those reported in our 
study, as previous research has shown that repeated ozone exposure over several days leads to the attenuation of pulmonary responses [12,51,52].

In summary, we showed that acute ozone exposure increased pulmonary oxidant status and upregulated the molecular control of mitophagy, and that in female offspring these responses were exacerbated by a perinatal HFD. Considering the current obesity pandemic and a substantial part of the urban population being exposed to harmful ozone levels, investments should be made into improving early life nutrition and reducing exposure to air pollution to protect society against adverse health effects.

\section{Acknowledgements}

The authors would like to thank Christy Tulen for providing real-time qPCR primers and Western blot antibodies. The authors also thank the U.S. Environmental Protection Agency's late Christopher Gordon for assisting in the study conceptualization and design; Pamela Phillips for animal assignment, handling, and care during experimentation; and Mette C. Schladweiler for performing the final necropsies. 


\section{References}

1. Snow, S.J.; Broniowska, K.; Karoly, E.D.; Henriquez, A.R.; Phillips, P.M.; Ledbetter, A.D.; Schladweiler, M.C.; Miller, C.N.; Gordon, C.J.; Kodavanti, U.P. Offspring susceptibility to metabolic alterations due to maternal high-fat diet and the impact of inhaled ozone used as a stressor. Scientific reports 2020, 10, 1-18.

2. Organization, W.H. Global status report on noncommunicable diseases 2014; World Health Organization: 2014.

3. Eurostat Data Browser. Available online: https://ec.europa.eu/eurostat/databrowser/view/hlth_ehis_bm1e/default/table?lang=en (accessed on 03 May 2021).

4. Eriksson, J.G.; Sandboge, S.; Salonen, M.K.; Kajantie, E.; Osmond, C. Long-term consequences of maternal overweight in pregnancy on offspring later health: findings from the Helsinki Birth Cohort Study. Annals of medicine 2014, 46, 434-438.

5. Gaillard, R.; Steegers, E.A.; Duijts, L.; Felix, J.F.; Hofman, A.; Franco, O.H.; Jaddoe, V.W. Childhood cardiometabolic outcomes of maternal obesity during pregnancy: the Generation R Study. Hypertension 2014, 63, 683-691.

6. Hochner, H.; Friedlander, Y.; Calderon-Margalit, R.; Meiner, V.; Sagy, Y.; Avgil-Tsadok, M.; Burger, A.; Savitsky, B.; Siscovick, D.S.; Manor, O. Associations of Maternal Prepregnancy Body Mass Index and Gestational Weight Gain With Adult Offspring Cardiometabolic Risk FactorsClinical Perspective: The Jerusalem Perinatal Family Follow-Up Study. Circulation 2012, 125, 1381-1389.

7. Yu, Z.; Han, S.; Zhu, J.; Sun, X.; Ji, C.; Guo, X. Pre-pregnancy body mass index in relation to infant birth weight and offspring overweight/obesity: a systematic review and metaanalysis. Plos One 2013, 8, e61627.

8. Forno, E.; Young, O.M.; Kumar, R.; Simhan, H.; Celedón, J.C. Maternal obesity in pregnancy, gestational weight gain, and risk of childhood asthma. Pediatrics 2014, 134, e535-e546.

9. Zugna, D.; Galassi, C.; Annesi-Maesano, I.; Baïz, N.; Barros, H.; Basterrechea, M.; Correia, S.; Duijts, L.; Esplugues, A.; Fantini, M.P. Maternal complications in pregnancy and wheezing in early childhood: a pooled analysis of 14 birth cohorts. International journal of epidemiology 2015, 44, 199-208.

10. Griffiths, P.S.; Walton, C.; Samsell, L.; Perez, M.K.; Piedimonte, G. Maternal high-fat hypercaloric diet during pregnancy results in persistent metabolic and respiratory abnormalities in offspring. Pediatric research 2016, 79, 278-286.

11. MacDonald, K.D.; Moran, A.R.; Scherman, A.J.; McEvoy, C.T.; Platteau, A.S. Maternal highfat diet in mice leads to innate airway hyperresponsiveness in the adult offspring. Physiological reports 2017, 5, e13082.

12. Brown, J.S.; Bateson, T.F.; McDonnell, W.F. Effects of exposure to 0.06 ppm ozone on FEV1 in humans: a secondary analysis of existing data. Environ Health Persp 2008, 116, 10231026.

13. Folinsbee, L.J.; Horstman, D.H.; Kehrl, H.R.; Harder, S.; Abdul-Salaam, S.; Ives, P.J. Respiratory responses to repeated prolonged exposure to $0.12 \mathrm{ppm}$ ozone. American journal of respiratory and critical care medicine 1994, 149, 98-105. 
14. Kim, C.S.; Alexis, N.E.; Rappold, A.G.; Kehrl, H.; Hazucha, M.J.; Lay, J.C.; Schmitt, M.T.; Case, M.; Devlin, R.B.; Peden, D.B. Lung function and inflammatory responses in healthy young adults exposed to $0.06 \mathrm{ppm}$ ozone for 6.6 hours. American journal of respiratory and critical care medicine 2011, 183, 1215-1221.

15. Que, L.G.; Stiles, J.V.; Sundy, J.S.; Foster, W.M. Pulmonary function, bronchial reactivity, and epithelial permeability are response phenotypes to ozone and develop differentially in healthy humans. Journal of applied physiology 2011, 111, 679-687.

16. Schelegle, E.S.; Morales, C.A.; Walby, W.F.; Marion, S.; Allen, R.P. 6.6-hour inhalation of ozone concentrations from 60 to 87 parts per billion in healthy humans. American journal of respiratory and critical care medicine 2009, 180, 265-272.

17. European Environment Agency. Available online: https://www.eea.europa.eu/data-andmaps/indicators/exceedance-of-air-quality-limit-2/assessment (accessed on 03 May 2021).

18. Gordon, C.; Phillips, P.; Johnstone, A.; Schmid, J.; Schladweiler, M.; Ledbetter, A.; Snow, S.; Kodavanti, U. Effects of maternal high-fat diet and sedentary lifestyle on susceptibility of adult offspring to ozone exposure in rats. Inhalation toxicology 2017, 29, 239-254.

19. Snow, S.J.; Phillips, P.M.; Ledbetter, A.; Johnstone, A.F.; Schladweiler, M.C.; Gordon, C.J.; Kodavanti, U.P. The influence of maternal and perinatal high-fat diet on ozone-induced pulmonary responses in offspring. Journal of Toxicology and Environmental Health, Part $A$ 2019, 82, 86-98.

20. Ruijter, J.; Ramakers, C.; Hoogaars, W.; Karlen, Y.; Bakker, O.; Van den Hoff, M.; Moorman, A. Amplification efficiency: linking baseline and bias in the analysis of quantitative PCR data. Nucleic acids research 2009, 37, e45-e45.

21. Schneider, C.A.; Rasband, W.S.; Eliceiri, K.W. NIH Image to ImageJ: 25 years of image analysis. Nature methods 2012, 9, 671-675.

22. Ruijter, J.M.; Thygesen, H.H.; Schoneveld, O.J.; Das, A.T.; Berkhout, B.; Lamers, W.H. Factor correction as a tool to eliminate between-session variation in replicate experiments: application to molecular biology and retrovirology. Retrovirology 2006, 3, 1-8.

23. Benjamini, Y.; Hochberg, Y. Controlling the false discovery rate: a practical and powerful approach to multiple testing. Journal of the Royal statistical society: series $B$ (Methodological) 1995, 57, 289-300.

24. Rikka, S.; Quinsay, M.N.; Thomas, R.L.; Kubli, D.A.; Zhang, X.; Murphy, A.N.; Gustafsson, Å.B. Bnip3 impairs mitochondrial bioenergetics and stimulates mitochondrial turnover. Cell Death \& Differentiation 2011, 18, 721-731.

25. Schweers, R.L.; Zhang, J.; Randall, M.S.; Loyd, M.R.; Li, W.; Dorsey, F.C.; Kundu, M.; Opferman, J.T.; Cleveland, J.L.; Miller, J.L. NIX is required for programmed mitochondrial clearance during reticulocyte maturation. Proceedings of the National Academy of Sciences 2007, 104, 19500-19505.

26. Matsuda, N.; Sato, S.; Shiba, K.; Okatsu, K.; Saisho, K.; Gautier, C.A.; Sou, Y.-s.; Saiki, S.; Kawajiri, S.; Sato, F. PINK1 stabilized by mitochondrial depolarization recruits Parkin to damaged mitochondria and activates latent Parkin for mitophagy. Journal of Cell Biology 2010, 189, 211-221.

27. Chuang, G.C.; Yang, Z.; Westbrook, D.G.; Pompilius, M.; Ballinger, C.A.; White, C.R.; Krzywanski, D.M.; Postlethwait, E.M.; Ballinger, S.W. Pulmonary ozone exposure induces 
vascular dysfunction, mitochondrial damage, and atherogenesis. American Journal of Physiology-Lung Cellular and Molecular Physiology 2009, 297, L209-L216.

28. Li, F.; Wiegman, C.; Seiffert, J.M.; Zhu, J.; Clarke, C.; Chang, Y.; Bhavsar, P.; Adcock, I.; Zhang, J.; Zhou, X. Effects of $\mathrm{N}$-acetylcysteine in ozone-induced chronic obstructive pulmonary disease model. Plos One 2013, 8, e80782.

29. Lam, H.C.; Baglini, C.V.; Lope, A.L.; Parkhitko, A.A.; Liu, H.-J.; Alesi, N.; Malinowska, I.A.; Ebrahimi-Fakhari, D.; Saffari, A.; Jane, J.Y. p62/SQSTM1 cooperates with hyperactive mTORC1 to regulate glutathione production, maintain mitochondrial integrity, and promote tumorigenesis. Cancer research 2017, 77, 3255-3267.

30. Deffieu, M.; Bhatia-Kiššová, I.; Salin, B.; Galinier, A.; Manon, S.; Camougrand, N. Glutathione participates in the regulation of mitophagy in yeast. J Biol Chem 2009, 284, 14828-14837.

31. Okamoto, K.; Kondo-Okamoto, N.; Ohsumi, Y. Mitochondria-anchored receptor Atg32 mediates degradation of mitochondria via selective autophagy. Developmental cell 2009, 17, 87-97.

32. Kleeberger, S.R.; Levitt, R.C.; Zhang, L.-Y.; Longphre, M.; Harkema, J.; Jedlicka, A.; Eleff, S.M.; DiSilvestre, D.; Holroyd, K.J. Linkage analysis of susceptibility to ozone-induced lung inflammation in inbred mice. Nature genetics 1997, 17, 475-478.

33. Prows, D.R.; Shertzer, H.G.; Daly, M.J.; Sidman, C.L.; Leikauf, G.D. Genetic analysis of ozoneinduced acute lung injury in sensitive and resistant strains of mice. Nature genetics 1997, 17, 471-474.

34. Li, F.; Xu, M.; Wang, M.; Wang, L.; Wang, H.; Zhang, H.; Chen, Y.; Gong, J.; Zhang, J.J.; Adcock, I.M. Roles of mitochondrial ROS and NLRP3 inflammasome in multiple ozone-induced lung inflammation and emphysema. Respiratory research 2018, 19, 1-12.

35. Wiegman, C.H.; Michaeloudes, C.; Haji, G.; Narang, P.; Clarke, C.J.; Russell, K.E.; Bao, W.; Pavlidis, S.; Barnes, P.J.; Kanerva, J. Oxidative stress-induced mitochondrial dysfunction drives inflammation and airway smooth muscle remodeling in patients with chronic obstructive pulmonary disease. J Allergy Clin Immun 2015, 136, 769-780.

36. Xu, M.; Wang, L.; Wang, M.; Wang, H.; Zhang, H.; Chen, Y.; Wang, X.; Gong, J.; Zhang, J.; Adcock, I.M. Mitochondrial ROS and NLRP3 inflammasome in acute ozone-induced murine model of airway inflammation and bronchial hyperresponsiveness. Free radical research 2019, 53, 780-790.

37. Onishi, M.; Yamano, K.; Sato, M.; Matsuda, N.; Okamoto, K. Molecular mechanisms and physiological functions of mitophagy. The EMBO Journal 2021, 40, e104705.

38. Pickles, S.; Vigié, P.; Youle, R.J. Mitophagy and quality control mechanisms in mitochondrial maintenance. Current Biology 2018, 28, R170-R185.

39. Williams, J.A.; Ding, W.-X. A mechanistic review of mitophagy and its role in protection against alcoholic liver disease. Biomolecules 2015, 5, 2619-2642.

40. Tang, C.; Han, H.; Yan, M.; Zhu, S.; Liu, J.; Liu, Z.; He, L.; Tan, J.; Liu, Y.; Liu, H. PINK1PRKN/PARK2 pathway of mitophagy is activated to protect against renal ischemiareperfusion injury. Autophagy 2018, 14, 880-897.

41. Chourasia, A.H.; Tracy, K.; Frankenberger, C.; Boland, M.L.; Sharifi, M.N.; Drake, L.E.; Sachleben, J.R.; Asara, J.M.; Locasale, J.W.; Karczmar, G.S. Mitophagy defects arising from 
BNip3 loss promote mammary tumor progression to metastasis. EMBO reports 2015,16 , 1145-1163.

42. Fuentes, N.; Nicoleau, M.; Cabello, N.; Montes, D.; Zomorodi, N.; Chroneos, Z.C.; Silveyra, P. $17 \beta$-Estradiol affects lung function and inflammation following ozone exposure in a sexspecific manner. American Journal of Physiology-Lung Cellular and Molecular Physiology 2019, 317, L702-L716.

43. Saben, J.L.; Boudoures, A.L.; Asghar, Z.; Thompson, A.; Drury, A.; Zhang, W.; Chi, M.; Cusumano, A.; Scheaffer, S.; Moley, K.H. Maternal metabolic syndrome programs mitochondrial dysfunction via germline changes across three generations. Cell reports 2016, 16, 1-8.

44. Bruce, K.D.; Cagampang, F.R.; Argenton, M.; Zhang, J.; Ethirajan, P.L.; Burdge, G.C.; Bateman, A.C.; Clough, G.F.; Poston, L.; Hanson, M.A. Maternal high-fat feeding primes steatohepatitis in adult mice offspring, involving mitochondrial dysfunction and altered lipogenesis gene expression. Hepatology 2009, 50, 1796-1808.

45. Larsen, T.D.; Sabey, K.H.; Knutson, A.J.; Gandy, T.C.; Louwagie, E.J.; Lauterboeck, L.; Mdaki, K.S.; Baack, M.L. Diabetic pregnancy and maternal high-fat diet impair mitochondrial dynamism in the developing fetal rat heart by sex-specific mechanisms. International journal of molecular sciences 2019, 20, 3090.

46. Hatch, G.E.; McKee, J.; Brown, J.; McDonnell, W.; Seal, E.; Soukup, J.; Slade, R.; Crissman, K.; Devlin, R. Biomarkers of dose and effect of inhaled ozone in resting versus exercising human subjects: Comparison with resting rats. Biomarker insights 2013, 8, BMI. S11102.

47. Bell, M.R. Comparing postnatal development of gonadal hormones and associated social behaviors in rats, mice, and humans. Endocrinology 2018, 159, 2596-2613.

48. Brown, J.; Bowman, C. Integrated science assessment for ozone and related photochemical oxidants. Washington, DC: US Environmental Protection Agency 2013.

49. Kodavanti, U.P.; Ledbetter, A.D.; Thomas, R.F.; Richards, J.E.; Ward, W.O.; Schladweiler, M.C.; Costa, D.L. Variability in ozone-induced pulmonary injury and inflammation in healthy and cardiovascular-compromised rat models. Inhalation toxicology 2015, 27, 39-53.

50. Miller, D.B.; Karoly, E.D.; Jones, J.C.; Ward, W.O.; Vallanat, B.D.; Andrews, D.L.; Schladweiler, M.C.; Snow, S.J.; Bass, V.L.; Richards, J.E. Inhaled ozone (O3)-induces changes in serum metabolomic and liver transcriptomic profiles in rats. Toxicology and applied pharmacology 2015, 286, 65-79.

51. Zhu, R.; Wang, Y.; Zhang, L.; Guo, Q. Oxidative stress and liver disease. Hepatology Research 2012, 42, 741-749.

52. Nassir, F.; Ibdah, J.A. Role of mitochondria in nonalcoholic fatty liver disease. International journal of molecular sciences 2014, 15, 8713-8742. 


\section{Supplement}

Table S1. Primer sequences for real-time quantitative PCR.

\begin{tabular}{|c|c|c|}
\hline Gene & Forward primer (5'-3') & Reverse primer $\left(5^{\prime}-3^{\prime}\right)$ \\
\hline \multicolumn{3}{|c|}{ Reference genes } \\
\hline Tubab1b & $\overline{\text { AGCGCAGCATCCAGTTTGT }}$ & CTGTGGTGTTGCTCAGCATAGA \\
\hline$R p / 13 a$ & GCGGATGAACACCAACCCGT & CAGCCTGGCCTCTTTTGGTCT \\
\hline Ppia & TCCATGGCAAATGCTGGACCAA & CCTGGACCCAAAACGCTCCA \\
\hline \multicolumn{3}{|c|}{ Antioxidants } \\
\hline Cat & GAATGGCTATGGCTCACACA & CAAGTTTTTGATGCCCTGGT \\
\hline Sod1 & CGAGCATGGGTTCCATGTC & CTGGACCGCCATGTTTCTTAG \\
\hline Sod 2 & ATTAACGCGCAGATCATGCA & CCTCGGTGACGTTCAGATTGT \\
\hline \multicolumn{3}{|c|}{ Mitophagy } \\
\hline Pink1 & CCAAACACCTTGGCCTTCTA & CTTAAGATGGCTTCGCTGGA \\
\hline Bnip3 & CAGAGCGGCGAGGAGAACCTGCAG & GCTGCTCCCATTCCCATTGCTGAAG \\
\hline Bnip3l & AGGCTAACCTGCAGCACAGT & CACTGCCGATGAAACTGCTA \\
\hline \multicolumn{3}{|c|}{ Mitochondrial biogenesis } \\
\hline Ppargc1a & GGGACATGTGCAGCCAAGACT & GATCTGGGCAAAGAGGCTGGT \\
\hline Ppargc1b & AGCGCTTCGAGGTGTTTGGT & TTCTCAGGGTAGCGCCGTTC \\
\hline Tfam & AATTGCAGCCATGTGGAGGGAG & GCCGGGCTTCCTTCTCTAAGC \\
\hline \multicolumn{3}{|c|}{ Mitochondrial fusion } \\
\hline Mfn1 & CGGAGGCATATGAAAGTGGC & ССАTCAGTTCCСTCCACACT \\
\hline Mfn2 & TTGACTCCAGCCATGTCCAT & GGTGACGATGGAGTTGCATC \\
\hline Opa1 & CAGCTGGCAGAAGATCTCAAG & CATGAGCAGGATTTTGACACC \\
\hline \multicolumn{3}{|c|}{ Mitochondrial fission } \\
\hline Drp1 & TGGAGATGGTGGTCAGGAAC & TTTCGTGCAACTGGAACTGG \\
\hline Fis1 & ATGGATGCCCAGAGATGAAG & ACGATGCCTCTACGGATGTC \\
\hline \multicolumn{3}{|c|}{$\underline{\text { Oxidative phosphorylation }}$} \\
\hline Ndufb3 & GAAGAAGCTTGCTGCACGAGG & ACGCAGCAAACCCCCATTTG \\
\hline$S d h b$ & GAACGGAGACAAGTACCTGGGG & GATGGTGTGGCAGCGGTAGA \\
\hline Cyc1 & ATGTTGCCACCTTCCTTCGCT & AGGACTGACCACTTATGCCGC \\
\hline Cox4i1 & CTGAAGGAGAAGGAGAAGG & CAGTGAAGCCGATGAAGA \\
\hline
\end{tabular}


Table S2. Immunoprecipitation buffer composition.

\begin{tabular}{ll}
\hline Component & Concentration \\
\hline Tris & $50 \mathrm{mM}$ \\
$\mathrm{NaCl}$ & $150 \mathrm{mM}$ \\
Glycerol & $10 \%(\mathrm{w} / \mathrm{v})$ \\
$\mathrm{NP}-40$ & $0.05 \%(\mathrm{w} / \mathrm{v})$ \\
Ethylenediaminetetraacetic acid (EDTA) & $1 \mathrm{mM}$ \\
cOmplete protease inhibitor cocktail* & $1 \mathrm{x}$ \\
PhosSTOP phosphatase inhibitor* & $1 \mathrm{x}$ \\
$\mathrm{pH}$ & 7.4 \\
\hline
\end{tabular}

*Roche, Basel, Switzerland

Table S3. Laemmli buffer (4x).

\begin{tabular}{ll}
\hline Component & Concentration \\
\hline Tris & $0.25 \mathrm{M}$ \\
Sodium dodecyl sulfate (SDS) & $8 \%(\mathrm{w} / \mathrm{v})$ \\
Glycerol & $40 \%(\mathrm{w} / \mathrm{v})$ \\
Dithiothreitol (DTT) & $0.4 \mathrm{M}$ \\
Bromophenol Blue & $0.02 \%(\mathrm{w} / \mathrm{v})$ \\
$\mathrm{pH}$ & 6.8 \\
\hline
\end{tabular}

Table S4. Western blot primary antibodies.

\begin{tabular}{lll}
\hline Antigen & Dilution & Supplier \\
\hline BNIP3 & $1: 1000$ & Cell Signaling Technology \\
BNIP3L & $1: 1000$ & Cell Signaling Technology \\
SOD2 & $1: 1000$ & Santa Cruz Biotechnology \\
OXPHOS I-V & $1: 1000$ & Abcam \\
\hline
\end{tabular}

Table S5. Western blot secondary antibodies.

\begin{tabular}{llll}
\hline Primary antibody & Secondary antibody & Dilution & Supplier \\
\hline BNIP3 & Anti-rabbit & $1: 10,000$ & Vector Laboratories \\
BNIP3L & Anti-rabbit & $1: 10,000$ & Vector Laboratories \\
SOD2 & Anti-mouse & $1: 10,000$ & Vector Laboratories \\
OXPHOS I-V & Anti-mouse & $1: 10,000$ & Vector Laboratories \\
\hline
\end{tabular}




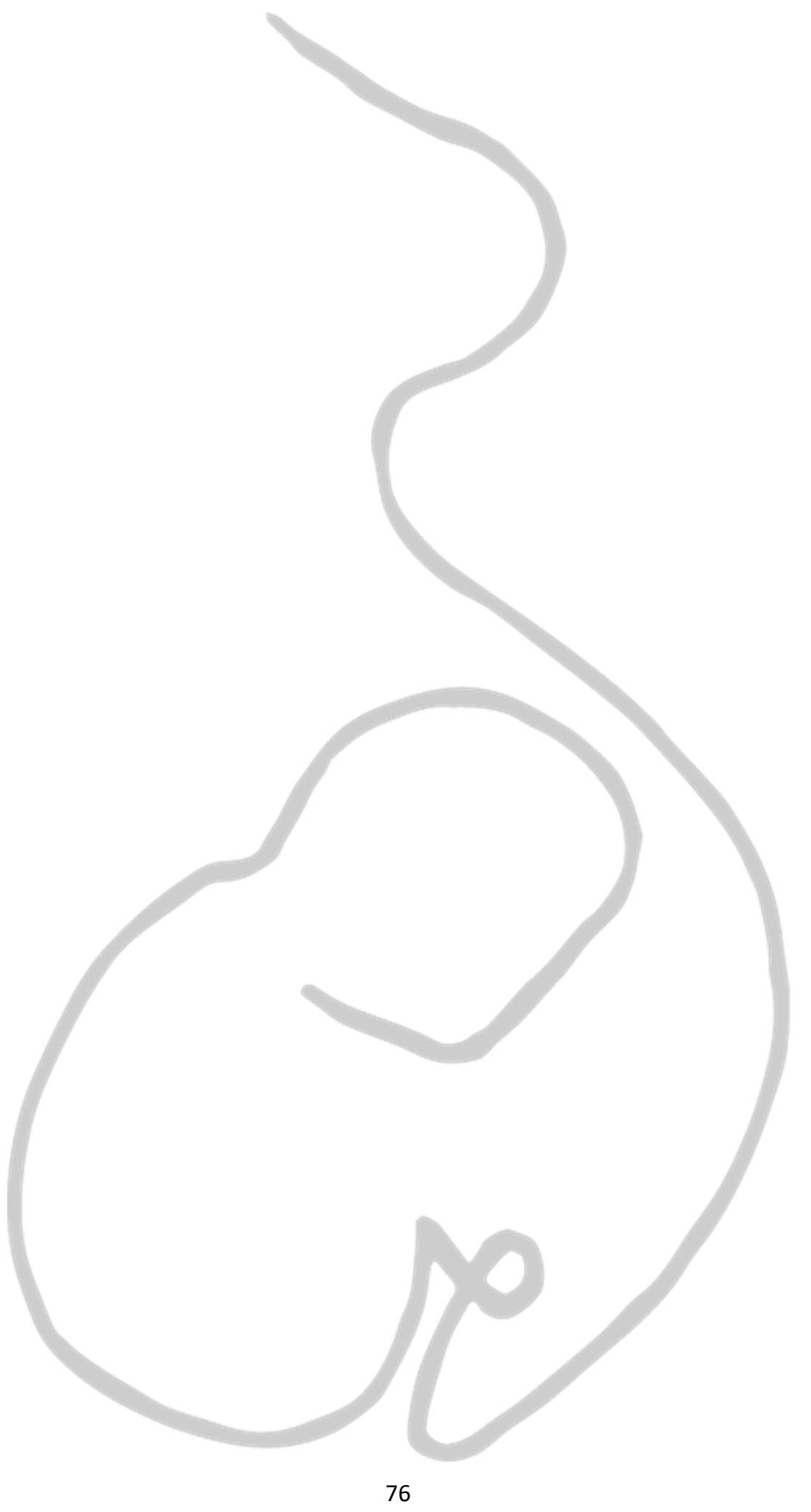




\section{Chapter 4}

\section{Perinatal fish oil diet reduces ex vivo cytokine levels of polarizing $\mathrm{CD}^{+} \mathrm{T}$ cells and induces anemia with microvesicular steatosis in mouse offspring}

Sven H. Rouschop, Laura Evers, Lou Maas, Marion Gijbels, Shahab Jolani, Wilfred Germeraad, Dennis M. Meesters, Ronit Shiri-Sverdlov, Frederik J. van Schooten, Antoon Opperhuizen, Roger W. Godschalk

In preparation for submission 


\begin{abstract}
Maternal supplementation with n-3 polyunsaturated fatty acids (PUFAs) during pregnancy is associated with reduced risk of developing asthma in the offspring. However, the mechanisms behind this association are unclear, including the role of progeny $\mathrm{CD} 4^{+} \mathrm{T}$ cells, which are essential to asthma pathology. Therefore, this study examined the effect of a perinatal n-3 PUFA-rich fish oil diet (FOD) on ex vivo polarization of $\mathrm{CD} 4^{+} \mathrm{T}$ cells in mouse offspring. For this purpose, dams received either an FOD or a control diet (CD) during gestation and lactation. At the end of lactation, offspring splenic naïve $\mathrm{CD}^{+}{ }^{+} \mathrm{T}$ cells were isolated and polarized ex vivo towards Th1 or Th2 lineage. During polarization, $\mathrm{CD}^{+}{ }^{+}$cells were examined for secretion and gene expression of Th-specific cytokines and transcription factors. The perinatal FOD significantly reduced extracellular levels of IL-4, IL-5, IL-13, IFN- $\gamma$ and IL-10 during Th1 polarization, and IL-5, IL-13 and IL-10 during Th2 polarization. The perinatal FOD affected mRNA expression of Th-specific cytokines and transcription factors as well, but these changes were less consistent than the effects observed for extracellular cytokine levels. In addition, the perinatal FOD unexpectedly induced anemia in both dams and offspring, and microvesicular steatosis in offspring liver. These findings suggest that perinatal exposure to high levels of n-3 PUFAs may have both beneficial and adverse effects in the offspring and thus that dosages should be carefully considered for future studies.
\end{abstract}




\section{Introduction}

Allergic asthma is a chronic inflammatory airway disorder, which is characterized by airway hyperreactivity and reversible airway obstruction. Asthma symptoms include shortness of breath, cough, wheezing, chest tightness, and a decreased expiratory airflow. Despite a growing knowledge of asthma, the prevalence of asthma has increased over the past few decades leading to current prevalences of $7.8 \%$ in the United States, $9.4 \%$ in the United Kingdom, and $5.4 \%$ in the European Union [1-4]. Concurrently, consumption of $n-3$ polyunsaturated fatty acids (PUFAs) has decreased, whereas consumption of $n-6$ PUFAs has increased, resulting in the previously formulated hypothesis by Black and Sharpe that the increasing prevalence of asthma and other allergic diseases may be explained by changes in the $n-6: n-3$ ratio of the diet [5]. More recently, epidemiologic studies have indeed found associations between low maternal intake of n-3 PUFAs and increased risk of asthma and asthma-related symptoms in offspring [6-8]. Moreover, intervention studies have shown that maternal supplementation with n-3 PUFAs and n-3 PUFA-rich fish oils during pregnancy may reduce the risk of offspring being diagnosed with asthma, having asthma medication prescribed, or experiencing asthma symptoms [9-11].

For the development of asthma and other allergies, sensitization to allergens is crucial. Sensitization to allergens is thought to occur early in life and involves antigen-induced polarization of $\mathrm{CD} 4^{+} \mathrm{T}$ cells $[12,13]$. Upon inhalation, the allergen is processed by a dendritic cell, which then displays allergen-derived antigens on its surface, becoming an antigenpresenting cell (APC), and presents the antigen to naïve $C D 4^{+} \mathrm{T}$ cells in the spleen or lymph nodes. In allergic individuals, naïve $\mathrm{CD}^{+} \mathrm{T}$ cells will predominantly polarize into $\mathrm{T}$ helper (Th) 2 cells, subsequently leading to hallmark features of asthma, such as B cell isotype switching to IgE, mast cell differentiation and maturation, and eosinophil maturation and survival [13]. However, naïve $C D 4^{+} T$ cells may also polarize into other Th subsets, such as Th1, Th17 and regulatory T (Treg) cells. Each of these Th lineages has its own polarization pathway, involving expression of Th-specific transcription factors and cytokines. For Th1 cells, transcription factor T-bet induces expression of cytokine IFN- $\gamma$, whereas for Th2 cells, transcription factor Gata3 promotes expression of cytokines IL-4, IL-5, and IL-13. For Th17 cells, secretion of cytokine IL-17 is induced by transcription factor RORyt, and for Treg cells, transcription factor Foxp3 induces expression of cytokine IL-10 [14]. Each of these Th subsets and their signature transcription factors and cytokines have their own associations with asthma. For instance, compared with healthy individuals, asthma patients display increased pulmonary levels of Th2 cytokines IL-4 and IL-5 and Th17 cytokine IL-17, and decreased levels of Th1 cytokine IFN- $\gamma$ and Treg cytokine IL-10 [15-18]. 
Although $\mathrm{CD}^{+} \mathrm{T}$ cells are known to be important for asthma pathology, and despite maternal consumption of n-3 PUFAs being associated with asthma risk in offspring, the effects of maternal $n-3$ PUFAs on $\mathrm{CD}^{+} \mathrm{T}$ cells in offspring have not been studied. We therefore performed a study in which we investigated the effect of a perinatal n-3 PUFArich fish oil diet (FOD) on ex vivo polarization of $\mathrm{CD} 4^{+} \mathrm{T}$ cells in mouse offspring. In this study, dams received either an FOD or a control diet (CD) during gestation and lactation. At the end of lactation, offspring splenic naïve $\mathrm{CD}^{+}{ }^{+} \mathrm{T}$ cells were isolated and polarized ex vivo towards Th1 or Th2 lineage. During polarization, $\mathrm{CD}^{+} \mathrm{T}$ cells were examined for secretion and gene expression of Th-specific cytokines and transcription factors to assess the effect of a perinatal FOD on ex vivo polarization of $\mathrm{CD}^{+}{ }^{+} \mathrm{T}$ cells. In this paper, we show that a perinatal FOD reduced extracellular cytokine levels of both Th1- and Th2-polarizing $C D 4^{+} T$ cells isolated from offspring. Furthermore, unanticipated secondary effects were observed, with a perinatal FOD inducing not only anemia in both dams and offspring, but also microvesicular steatosis in offspring liver.

\section{Methods}

\section{Animal experiment}

Animals were housed in individually ventilated cages, at a temperature of $21^{\circ} \mathrm{C}$, relative air humidity of $55 \pm 10 \%, 12 / 12$-hour light/dark cycle, and with ad libitum access to feed and water. Specific pathogen-free BALB/cByJ females (Charles River, Écully, France) were densely co-housed in a female-only room for two weeks and subsequently exposed to male pheromones through bedding material to synchronize the females' estrus cycle (Lee-Boot and Whitten effect $[19,20])$. Females (eight weeks old) were then mated for a maximum of two consecutive nights and included in the experiment if they had a vaginal plug the morning after mating. Plug-positive females were randomly assigned to either a control diet (CD; Research Diets, New Brunswick, NJ, USA) or an FOD (Research Diets). Both diets were identical in composition, except for the fat source, which was soybean oil for the CD and menhaden oil combined with flaxseed oil for the FOD. Diets were designed to have minimal differences in total saturated fatty acids (SFA), total monounsaturated fatty acids (MUFA), and total PUFA, but opposing amounts of eicosapentaenoic acid (EPA), docosahexaenoic acid (DHA), total n-3 PUFAs and total n-6 PUFAs (Table 1). Detailed formulas for both diets are listed in Table S1. Diets started on gestational day 0 (GD; number of days after confirmation of vaginal plug) and continued throughout gestation and lactation. To prevent oxidation of the diet, the antioxidant tert-butylhydroquinone was added. Furthermore, the diets were stored in vacuum bags at $-20^{\circ} \mathrm{C}$ and the diets in animal cages were replaced with fresh feed three times per week. Bodyweight was assessed on GD 0, 7, and 14 for dams, and 
Table 1. Macronutrient and fatty acid composition of control diet (CD) and fish oil diet (FOD).

\begin{tabular}{lll}
\hline & CD & FOD \\
\hline Macronutrients & $\mathbf{( k c a l} \%)$ & $\mathbf{( k c a l} \%)$ \\
Fat & 12 & 12 \\
Protein & 26 & 26 \\
Carbohydrate & 62 & 62 \\
Fatty acids & $\mathbf{( g / k g )}$ & $\mathbf{( g / \mathbf { k g } )}$ \\
EPA & 0.00 & 1.92 \\
DHA & 0.00 & 1.39 \\
n-3 PUFA & 3.70 & 24.43 \\
n-6 PUFA & 25.80 & 6.45 \\
Total SFA & 7.54 & 6.11 \\
Total MUFA & 11.66 & 9.44 \\
Total PUFA & 29.53 & 31.54 \\
\hline
\end{tabular}

In vivo experiment

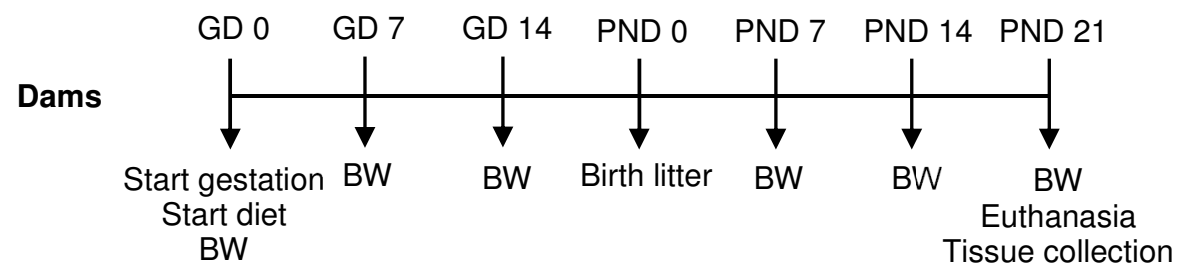

Offspring

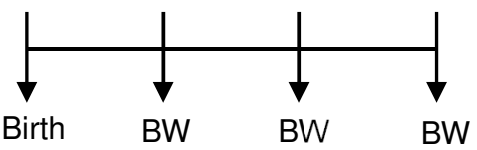

Euthanasia

Tissue collection

Figure 1. Experimental design of animal model. Dams received a control diet or fish oil diet during gestation and lactation. Body weight (BW) was measured on gestational day (GD) 0, 7 and 14 for dams, and on postnatal day (PND) 7, 14 and 21 for both dams and offspring. At the end of lactation (PND 21), both dams and offspring were euthanized and tissues were collected for further analyses. GD 0 and PND 0 are the first day of gestation and lactation, respectively.

on postnatal day (PND; number of days after birth of litter) 7, 14, and 21 for both dams and offspring. At the end of lactation, on PND 21, both dams and offspring were euthanized for tissue collection. Litters were not culled and both male and female offspring were used for assessment of all outcomes. Animal experiments were approved by the Animal Experiment 
Committee of Maastricht University (project license number AVD1070020198784). For a schematic overview of the animal model, see Figure 1.

\section{Animal tissue collection}

On PND 21, dams received a single subcutaneous dose of $0.05 \mathrm{mg} / \mathrm{kg}$ buprenorphine. Thirty minutes later, anesthesia was induced with $4-5 \%$ isoflurane and maintained with $1.5-2.5 \%$ isoflurane. After confirmation of full anesthesia, dams were exsanguinated via the vena cava and blood was collected in $\mathrm{K}_{2}$ EDTA tubes (BD Biosciences, Franklin Lakes, NJ, USA). Whole blood for hematological assessment was stored on ice and measured within a few hours. To obtain plasma, blood was centrifuged at 1,300 g for $15 \mathrm{~min}$ at $4^{\circ} \mathrm{C}$ and plasma was stored at $-80^{\circ} \mathrm{C}$ until fatty acid and ferritin analyses. Liver and spleen were stored in formalin for histology. Offspring was euthanized using cervical dislocation. Offspring spleen was collected in ice-cold PEB buffer (PBS, pH 7.2, 2 mM EDTA, 0.5\% (w/v) bovine serum albumin (BSA); prepared from MACS Rinsing Solution and MACS BSA Stock Solution; Miltenyi Biotec, Bergisch Gladbach, Germany) and directly used for enrichment of naïve $\mathrm{CD}^{+}{ }^{+} \mathrm{T}$ cells. Offspring liver was snap-frozen in liquid nitrogen and stored at $-80^{\circ} \mathrm{C}$ for gene expression analysis, or stored in formalin for histology.

\section{Enrichment of naïve $\mathrm{CD}^{+} \mathrm{T}$ cells}

Preparations of single-cell suspensions and enrichment of naïve $\mathrm{CD}^{+} \mathrm{T}$ cells were performed sterile and on ice. Freshly harvested spleens were transferred to $1 \mathrm{~mL}$ of fresh, ice-cold PEB buffer in a 24-well plate. Spleens were gently smashed with the thumb side of a syringe plunger and filtered through a $30 \mu \mathrm{m}$ cell strainer (Miltenyi Biotec). Naïve $\mathrm{CD} 4^{+} \mathrm{T}$ helper cells $\left(\mathrm{CD} 45^{+} \mathrm{CD} 3^{+} \mathrm{CD} 4^{+} \mathrm{CD} 62 \mathrm{~L}^{\text {high }} \mathrm{CD} 44^{\text {low }}\right)$ were enriched from single-cell suspensions using negative depletion with Naïve $\mathrm{CD} 4^{+} \mathrm{T}$ Cell Isolation Kit (Miltenyi Biotec) according to manufacturer's protocol and directly used for ex vivo polarization assay. Purity of naïve cells was determined to be $\geq 90 \%$ using flow cytometry (Figure S1). Less than $4 \%$ of enriched cells were effector T cells and $0 \%$ were memory cells. For flow cytometry, cells were stained with CD45-VioGreen, CD4-VioBlue, CD3-APC, CD62-PE, and CD44-VioBright FITC (all Miltenyi Biotec; catalog numbers listed in Table S2) according to manufacturer's protocol. Samples were measured with BD FACS Canto II and data were analyzed with BD FACSDiva Software v6.1.2 (BD Biosciences).

\section{Ex vivo polarization assay}

Naïve CD4 ${ }^{+}$T cells were seeded in 96 well-plates at $5 * 10^{4}$ cells/well in TexMACS medium (Miltenyi Biotec), supplemented with $10 \%$ (v/v) fetal bovine serum (Life Technologies), 0.01 
$\mathrm{mM}$ 2-mercaptoethanol, 1\% (v/v) Pen Strep (Life Technologies; final concentrations 100 $\mathrm{U} / \mathrm{ml}$ penicillin and $100 \mu \mathrm{g} / \mathrm{ml}$ streptomycin), Dynabeads Mouse T-activator CD3/CD28 (ThermoFisher Scientific, Waltham, MA, USA) at a 1:1 cell:bead ratio, $10 \mathrm{U} / \mathrm{ml} \mathrm{IL}-2$, and polarization-specific cytokines (i.e. $5 \mathrm{ng} / \mathrm{ml} \mathrm{IL-12} \mathrm{for} \mathrm{Th1} \mathrm{polarization} \mathrm{or} 5 \mathrm{ng} / \mathrm{ml} \mathrm{IL-4}$ for Th2 polarization). IL-2, IL-12 and IL-4 were purchased at Miltenyi Biotec (catalog numbers listed in Table S3). Cells were incubated in a humidified $\mathrm{CO}_{2}(5 \%)$ incubator at $37^{\circ} \mathrm{C}$ and harvested at $0,4,5$, and 6 days after seeding. Cells were centrifuged at $300 \mathrm{~g}$ for $5 \mathrm{~min}$, supernatant was stored at $-20^{\circ} \mathrm{C}$ for extracellular cytokine detection, and cell pellets were stored at $80^{\circ} \mathrm{C}$ for RNA isolation. For a schematic overview of the polarization assay, see Figure 2.

\section{Ex vivo polarization assay}

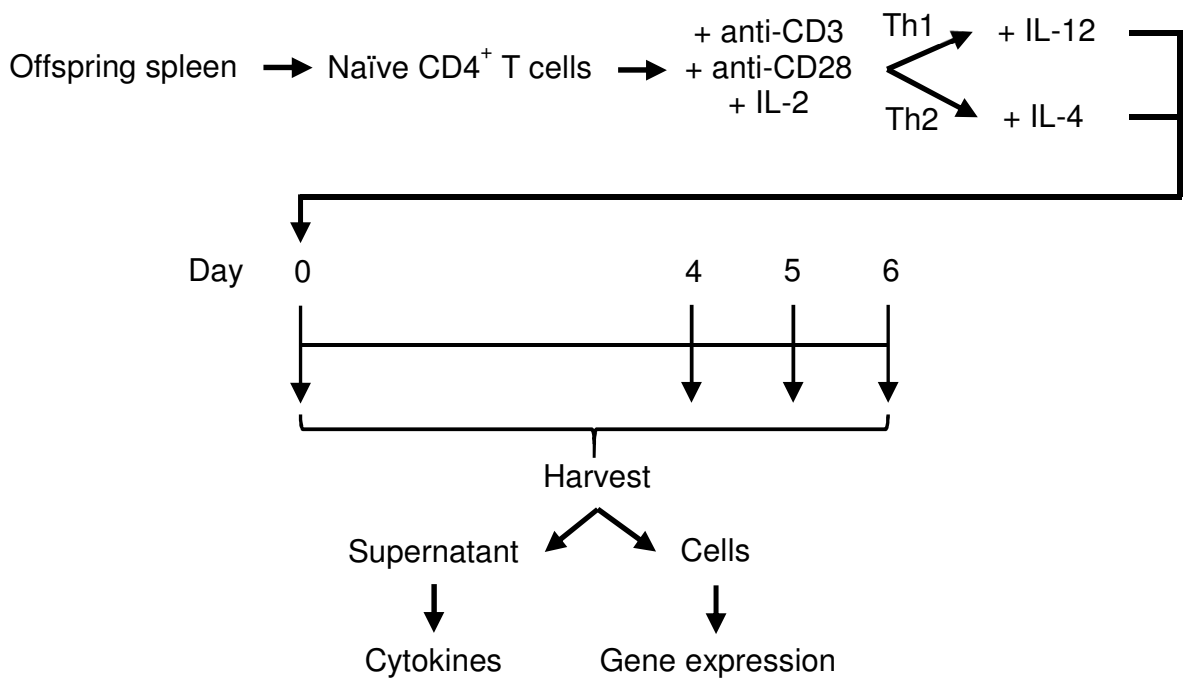

Figure 2. Experimental design of ex vivo polarization assay. Directly after euthanasia on postnatal day 21 , naïve $C D 4^{+} T$ cells were enriched from offspring spleen. $T$ cells were activated with antiCD3 and anti-CD28, and stimulated to polarize towards Th1 or Th2 lineage with IL-2 and IL-12 or with IL-2 and IL-4, respectively. Cells were cultured for six days and harvested on day 0, 4, 5 and 6 of polarization. In supernatant, Th-specific cytokines were measured. In cells, gene expression of Th-specific transcription factors and cytokines were measured.

\section{Extracellular cytokine concentrations and gene expression of $\mathrm{CD4}^{+} \mathrm{T}$ cells}

Extracellular cytokine levels of ex vivo polarizing $\mathrm{CD}^{+} \mathrm{T}$ cells were determined using a custom ProcartaPlex 6-plex assay (Thermo Fisher Scientific) according to manufacturer's protocol on a Luminex 100 system (Luminex Corporation, Austin, TX, USA). To assess gene 
expression of Th-specific transcription factors and cytokines, RNA was isolated from cell pellets using RNeasy Plus Mini Kit (Qiagen) according to manufacturer's protocol. cDNA was synthesized with iScript cDNA Synthesis Kit (Bio-Rad, Hercules, CA, USA) according to manufacturer's protocol. Real-time quantitative PCR (RT-qPCR) was performed using SensiMix SYBR \& Fluorescein Kit (Meridian Bioscience, Cincinnati, OH, USA) with $4.4 \mu \mathrm{l}$ of 1:10 diluted cDNA and $150 \mathrm{nM}$ of each target-specific primer (Table S4) in a total reaction volume of $10 \mu \mathrm{l}$ on a LightCycler 480 (Roche, Basel, Switzerland). Gene expression was quantified using LinRegPCR software version 2014.0 [21] and normalized using the geometric mean of expression levels of reference genes $\mathrm{Cd} 3 e$ and $\mathrm{Actb}$.

\section{Plasma fatty acids, plasma ferritin, hematology, and dietary iron}

Concentrations of 58 different free phospholipid fatty acids were measured by gas chromatography - triple quadrupole mass spectrometry in dam plasma according to Schött et al. [22]. Ferritin levels were assessed in dam plasma with Mouse Ferritin ELISA Kit (Crystal Chem, Elk Grove Village, IL, USA) according to manufacturer's protocol. Erythrocyte characteristics were determined in dam whole blood on a Sysmex XN-9000 automated flow cytometer (Sysmex, Kobe, Hyogo, Japan) at Maastricht University Medical Centre Central Diagnostic Laboratory. Dietary iron levels were determined by inductively coupled plasma optical emission spectrometry at NutriControl (Veghel, The Netherlands).

\section{Histology}

Formalin-preserved tissues were embedded in paraffin, cut into $4 \mu \mathrm{m}$ sections and stained with hematoxylin-eosin (HE), periodic acid-Schiff (PAS) or periodic acid-Schiff-diastase (PASD). Snap-frozen tissues were cut into $7 \mu \mathrm{m}$ sections and stained with Oil Red O (ORO).

\section{Gene expression in offspring liver}

For offspring liver gene expression, 50 - 100 mg snap-frozen tissue was homogenized in TRIReagent (Sigma-Aldrich, Saint Louis, MO, USA) and RNA was isolated using standard phenol/chloroform extraction. 300 ng RNA was converted into CDNA with the SensiFAST cDNA Synthesis Kit according to manufacturer's protocol. For RT-qPCR, SensiMix SYBR \& Fluorescein Kit (Meridian Bioscience) was used with $5 \mu$ of $7.5 x$ diluted cDNA and $300 \mathrm{nM}$ target-specific primers (Table S4) in a total reaction volume of $15 \mu \mathrm{l}$. Expression levels were quantified using LinRegPCR software version 2014.0 [21] and normalized with the geometric mean of Gapdh and Hprt expression levels. 


\section{Statistics}

All researchers were blinded for diet allocation up until data analysis. A priori power calculations determined that optimal group sizes would be 33 pups per group for two-sided $\alpha=0.05$ and power of 0.80 . To obtain this amount of pups, 36 dams per group started breeding procedures. In the end, seven vs. six dams (CD vs. FOD, respectively) gave birth to a viable litter, resulting in 35 vs. 27 pups (CD vs. FOD) that finished the experiment.

Based on density and Q-Q plots (with $Q-Q$ lines), data distributions were improved by $\log _{10^{-}}$ transformation for $\mathrm{CD}^{+} \mathrm{T}$ cell extracellular cytokine levels; by square root-transformation for $\mathrm{CD}^{+} \mathrm{T}$ cell gene expression; and by $\log _{2}$-transformation for offspring liver gene expression. Population characteristics were analyzed with unpaired, two-tailed t-test (for litter size at birth), Poisson regression with diet as factor (for gestational age at birth), or Fisher's exact test (for offspring sex and offspring mortality). Overall fatty acid profiles were visualized with principle component analysis using standardized fatty acid concentrations. The effect of FOD on individual and grouped fatty acid levels and on plasma ferritin levels was analyzed with unpaired, two-tailed t-tests. For offspring and dam body weight, offspring liver gene expression, and cytokine levels and gene expression assessed in the polarization assays, data were analyzed with the linear mixed-effects models. For these mixed-effects models, time and a diet*time interaction were used as fixed factors when assessing dam outcomes, whereas for offspring outcomes, sex, time and a diet*time interaction were used as fixed factors.

Results were graphically presented as boxplots, with boxes representing first quartile, median and third quartile, and whiskers representing minimum and maximum values within 1.5 times the interquartile range (IQR) below the first quartile or above the third quartile, respectively, and with points representing values outside the limits of the whiskers. $P<0.05$ was considered significant. All statistical analyses were performed in $R$ version 4.0.2 ( $R$ Foundation for Statistical Computing, Vienna, Austria) with nlme package version 3.1-152 for linear mixed-effects model. 


\section{Results}

\section{Perinatal FOD alters fatty acid profile in dams}

To study the effect of maternal n-3 PUFAs on ex vivo polarization of offspring CD4 ${ }^{+} \mathrm{T}$ cells, dams were fed an FOD or CD during gestation and lactation. First, to assess whether the perinatal FOD indeed changed maternal fatty acid status, 58 different fatty acids were measured in maternal plasma at the end of lactation. Principle component analysis of these
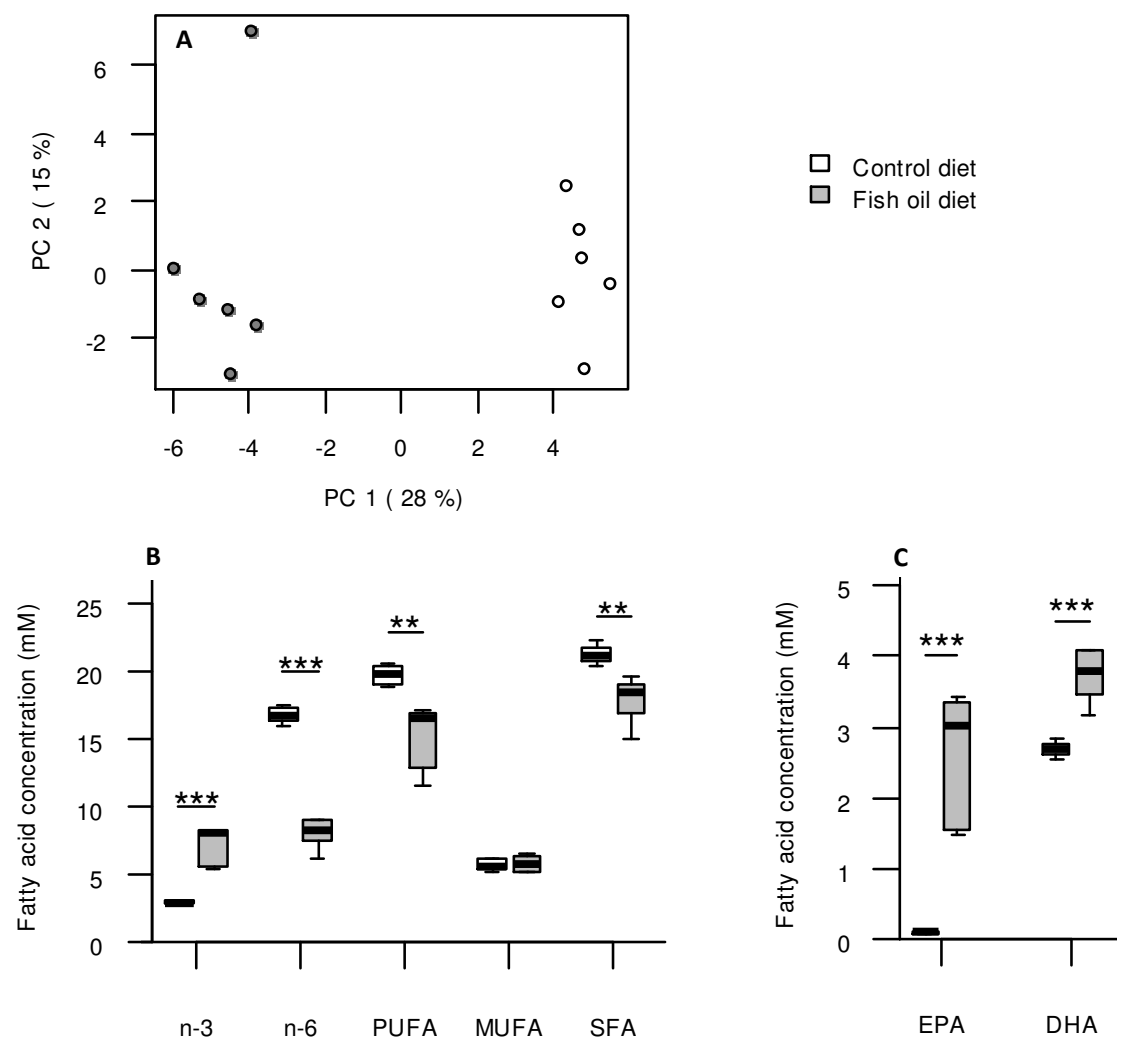

Figure 3. Perinatal fish oil diet (FOD) alters maternal plasma fatty acid profile. A) Principle component analysis of 58 fatty acids. B) Eicosapentanoic acid (EPA) and docosahexanoic acid (DHA) levels. C) Total $n-3$ fatty acids, total $n-6$ fatty acids, total polyunsaturated fatty acids (PUFA), total monounsaturated fatty acids (MUFA), and total saturated fatty acids (SFA) levels. Fatty acids were measured in dam plasma at the end of lactation (postnatal day 21), after dams received a control diet (CD) or FOD during gestation and lactation. Data are presented as A) scatter plot of the first and second principle component (PC), covering $28 \%$ and $15 \%$ of the total variance, respectively, with each dot representing one dam; or as boxplots ( $B$ and $C$ ). Statistical analysis by unpaired, twotailed t-test (B and C). $\mathrm{n}=6$ per group. ${ }^{* *} \mathrm{p}<0.01,{ }^{* * *} \mathrm{p}<0.001$. 
maternal plasma fatty acid data showed that FOD dams had a different overall fatty acid status than CD dams (Figure 3a). Furthermore, in accordance with the diet, FOD dams had significantly increased levels of EPA, DHA and total n-3 PUFAs, and significantly decreased levels of total n-6 PUFAs, compared with CD dams (Figure 3b-c). Although the FOD was designed to have similar levels of total PUFA, total MUFA and total SFA when compared with the CD, plasma levels of total PUFA and total SFA were significantly lower in FOD dams compared with CD dams (Figure 3c). Total MUFA levels were not different between both groups. Individual concentrations of all 58 measured fatty acids are listed in Table S5. Altogether, these findings indicate that a perinatal FOD significantly altered the maternal fatty acid profile.

\section{Perinatal FOD decreases offspring bodyweight during lactation}

To check whether the perinatal FOD affected the animals' general health, several baseline characteristics were assessed. As expected, no significant differences were observed between $C D$ and FOD animals for gestational duration, litter size, offspring sex and offspring mortality (Table 2). In addition to these characteristics, dam bodyweight was measured during gestation and lactation, and offspring bodyweight was measured during lactation. Both CD and FOD dams gained similar amounts of bodyweight during gestation and remained constant in bodyweight during lactation (Figure 4a-b). Offspring from both CD and FOD dams increased bodyweight during lactation, but for offspring from FOD dams, weight gain was significantly lower than for offspring from CD dams (Figure 4c). In summary, these findings show that a perinatal FOD decreased offspring bodyweight during lactation.

Table 2. Population characteristics. No significant differences were found between control diet (CD) and fish oil diet (FOD) for any of the parameters. Statistical analysis by Poission regression (gestational age at birth), unpaired, two-tailed t-test (litter size at birth), or Fisher's exact test (offspring sex and offspring deceased during lactation). All numbers represent count data, except litter size at birth, which represents mean and 95\% confidence interval (CI).

\begin{tabular}{lll}
\hline & CD & FOD \\
\hline Dams with viable litter & 7 & 6 \\
Gestational age at birth & & \\
19 days & 3 & 1 \\
20 days & 4 & 4 \\
21 days & 0 & 1 \\
Litter size at birth (mean $(95 \% \mathrm{Cl}))$ & $5.3(3.4-7.2)$ & $4.8(3.0-6.6)$ \\
Offspring born & 37 & 29 \\
Offspring sex (female/male) & $25 / 12$ & $15 / 14$ \\
Offspring deceased during lactation & 2 & 2 \\
Offspring included in ex vivo experiment & 35 & 27 \\
\hline
\end{tabular}



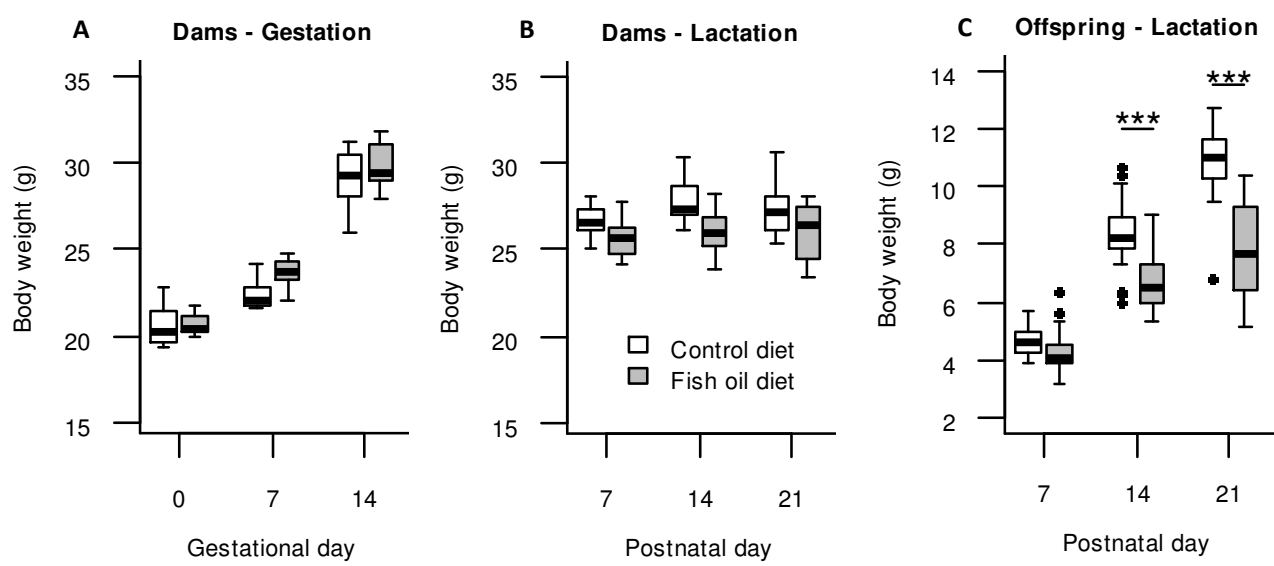

Figure 4. Perinatal fish oil diet (FOD) decreases offspring bodyweight during lactation. A) Dam bodyweight during gestation, $\mathrm{n}=7$ vs. 6 (control diet (CD) vs. FOD). B) Dam bodyweight during lactation, $n=7$ vs. 6 . C) Offspring bodyweight during lactation. $n=35$ vs. $27,{ }^{* * *} p<0.001$ for corresponding diet*day interaction. Dams received CD or FOD during gestation and lactation. Data are presented as boxplots. Statistical analysis by linear mixed-effect models.

\section{Perinatal FOD induces anemia in both dams and offspring}

To examine whether the decreased bodyweight in offspring from FOD dams was related to an underlying pathology, we performed a number of additional secondary analyses in a subgroup of dams and offspring. Since offspring from FOD dams appeared to be lethargic, tissues were first examined for signs of anemia. Indeed, spleens from FOD dams, as well as livers from offspring from FOD dams displayed extramedullary hematopoiesis, which is a clear indication of anemia, whereas no extramedullary hematopoiesis was observed in spleens from CD dams and livers from offspring from CD dams (Figure 5a-d). Anemia diagnosis was further supported by hematological assessment of two FOD dams and one CD dam, which showed that both FOD dams had lower red blood cell count, hemoglobin concentration and mean corpuscular hemoglobin, and higher relative distribution width, compared with CD (Table S6). In addition, both FOD dams had lower mean corpuscular volume than $C D$, suggesting that the FOD-induced anemia may be related to iron deficiency rather than a vitamin deficiency [23]. This suggestion was further examined by measuring different iron-related markers. First, iron levels were measured in both diets, but these did not differ between the FOD and CD (47 mg/kg diet for both CD and FOD). Moreover, iron levels in CD and FOD were above the levels advised for laboratory animals by the NRC (35 $\mathrm{mg} / \mathrm{kg}$ diet) [24]. In addition, serum ferritin concentration, which is a marker for total iron storage in the body, was measured in dam plasma. Although not statistically significant, ferritin levels tended to be lower in FOD dams than in CD dams (Figure 6a). Lastly, 


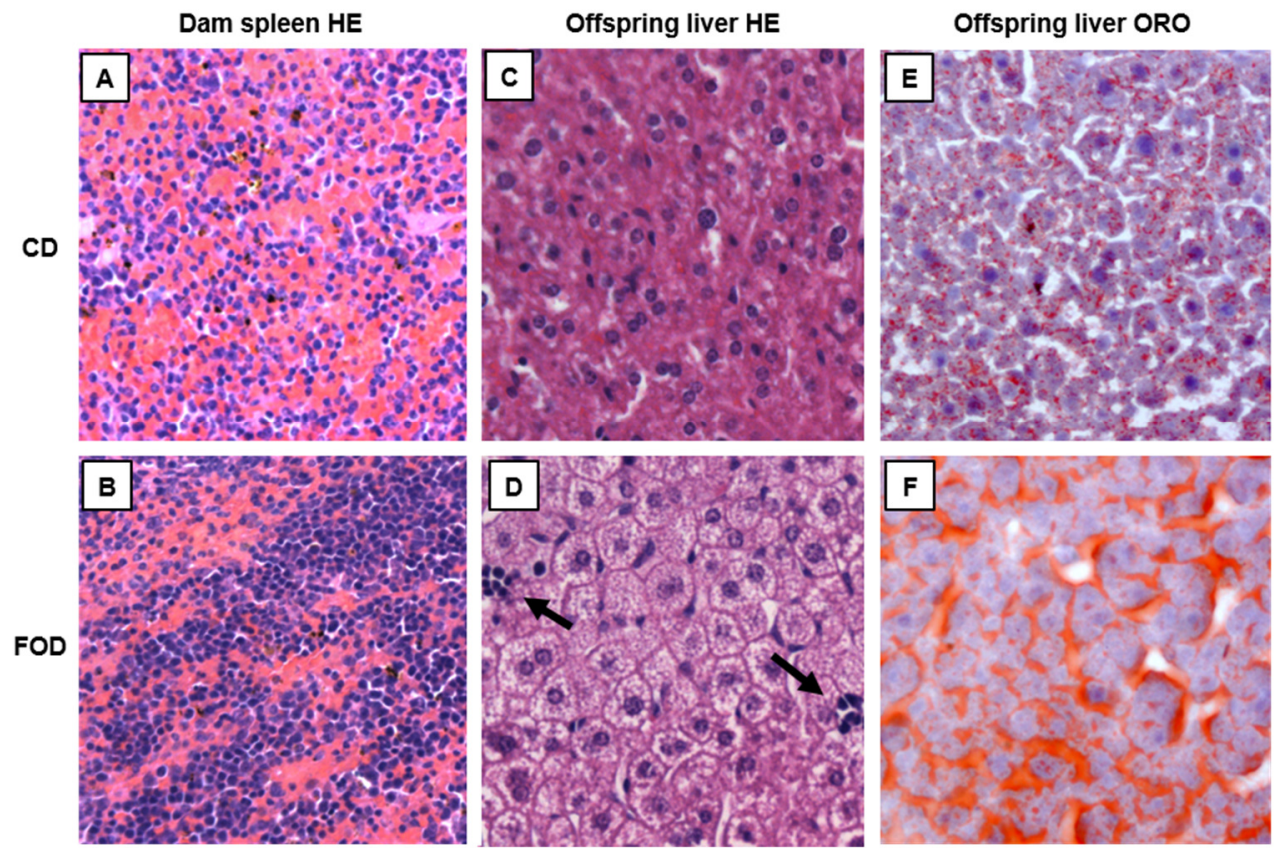

Figure 5. Perinatal fish oil diet (FOD) induces extramedullary hematopoiesis in dams and offspring, and hepatic microvesicular steatosis in offspring. A) Hematoxylin-eosin (HE)-stained spleen from control diet (CD)-fed dams. B) HE-stained spleen from FOD-fed dams showing extramedullary hematopoiesis (dark purple cells). C) HE-stained liver from offspring from CD-fed dams. D) HEstained liver from offspring from FOD-fed dams showing extramedullary hematopoiesis (dark cells at arrows) and microvesicular steatosis (sponge-like cells). E) Oil Red O (ORO)-stained liver from offspring from CD-fed dams. F) ORO-stained liver from offspring from FOD-fed dams showing increased lipid accumulation. Tissues were collected at the end of lactation (postnatal day 21) after dams received a CD or FOD during gestation and lactation. Images were taken at $200 x$ magnification. Representative images are shown, $n=3$ per group.

transcription levels of the hepcidin gene (Hamp), which is a master regulator of systemic iron metabolism [25], were measured in offspring liver, but no differences were found between offspring from FOD dams and offspring from CD dams (Figure 6b). Altogether, these data suggest that the perinatal FOD induced anemia in both dams and offspring.

\section{Perinatal FOD induces microvesicular steatosis in offspring liver}

During the examination of offspring liver for histological signs of anemia, an additional, unanticipated observation was made: livers of offspring from FOD dams displayed microvesicular steatosis, which was not present in livers of offspring from CD dams (Figure $5 c-d)$. Additional lipid staining of these tissues showed enhanced lipid accumulation in livers 


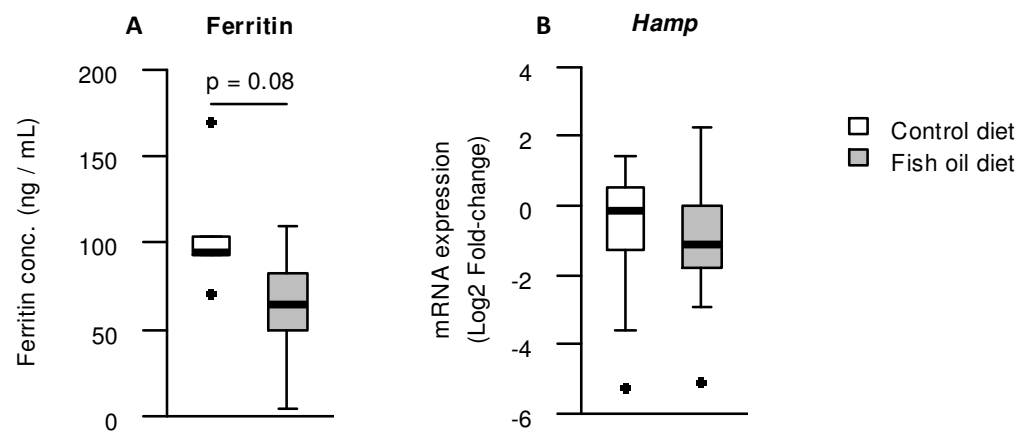

Figure 6. Effect of perinatal fish oil diet (FOD) on iron-related markers. A) Dam plasma ferritin levels, $n=5$ vs. 6 (control diet (CD) vs. FOD), statistical analysis by unpaired, two-tailed t-test. B) Gene expression of Hepcidin gene Hamp in offspring liver, $n=31$ vs. 24, statistical analysis by linear mixed-effect models. Both maternal plasma and offspring liver were collected at the end of lactation (on postnatal day 21) after dams received a CD or FOD during gestation and lactation. Data are presented as boxplots.

of offspring from FOD dams, whereas livers of offspring from CD dams only showed mild lipid accumulation (Figure 5e-f). No signs of inflammation, apoptosis or necrosis were found in livers of offspring from both CD and FOD dams. Furthermore, PAS staining of offspring livers showed no differences in hepatic glycogen levels between offspring from CD and FOD dams (results not shown). Histological examination of offspring heart, lung, thymus, kidney, pancreas, salivary gland, and gastrointestinal tract did not show any abnormalities (results not shown). In summary, these results indicate that a perinatal FOD induced microvesicular steatosis in offspring liver.

\section{Perinatal FOD reduces extracellular cytokine levels of ex vivo polarizing $\mathrm{CD}^{+}{ }^{+}$cells}

To examine the effect of a perinatal FOD on $\mathrm{CD}^{+} \mathrm{T}$ cell polarization, we set up an assay in which naïve $\mathrm{CD}^{+}{ }^{+} \mathrm{T}$ cells were isolated from offspring spleen at the end of lactation and polarized ex vivo towards Th1 or Th2 lineage. In this ex vivo polarization assay, Th1 polarization induced elevation of extracellular levels of IL-4, IL-5, IL-13, IFN- $\gamma$ and IL-10, with the largest elevation for IFN- $\gamma$. In addition, Th1 polarization increased mRNA expression of cytokine genes $/ 14,1 / 5, \| 13$, Ifng and $/ 110$, and of transcription factors Tbx21 and Foxp3. On the other hand, Th1 polarization decreased mRNA expression of transcription factor Gata3. Th1 polarization did not affect any Th17 marker (i.e., extracellular levels of IL-17a, and mRNA expression of $I / 17 a$ and Rorc). To study the effect of perinatal FOD on $\mathrm{CD}^{+} \mathrm{T}$ cell polarization, these polarization responses were compared between cells isolated from $C D$ offspring and cells isolated from FOD offspring. This showed that the perinatal FOD altered 
polarization-induced responses, with most profound effects on extracellular cytokine levels. Extracellular cytokine levels were significantly lower in the FOD group than the CD group for IFN- $\gamma$, IL-13 and IL-10 on polarization days 4, 5 and 6; for IL-5 on polarization days 4 and 6; and for IL-4 on polarization day 4 (Figure 7a-e). The perinatal FOD also affected polarization-induced changes in gene expression, but these effects were less consistent than the FOD effects on extracellular cytokine levels. mRNA levels of $/ / 4$, $/ 15$, and $/ / 13$ were significantly lower in the FOD group than the CD group on polarization day 4 , whereas expression of Gata3 was significantly higher in the FOD group than the CD group on polarization days 4 and 5, as was expression of Foxp3 on polarization day 4 (Figure 7f-j). Gene expression of Ifng, I/10 and Tbx21 were not affected by the perinatal FOD (Figure S2).

Th1 polarization assay
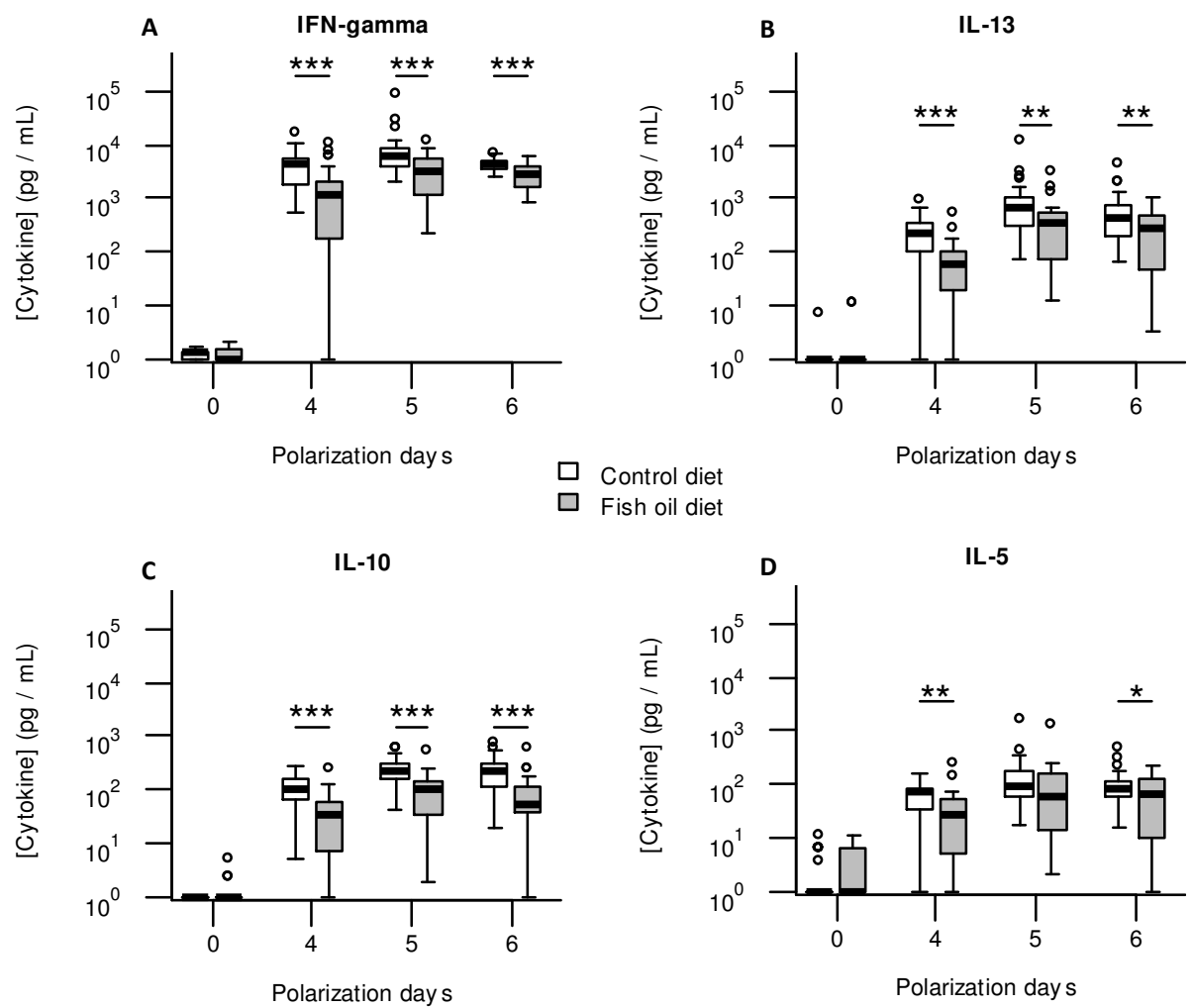

Figure 7 (continued on next page). 


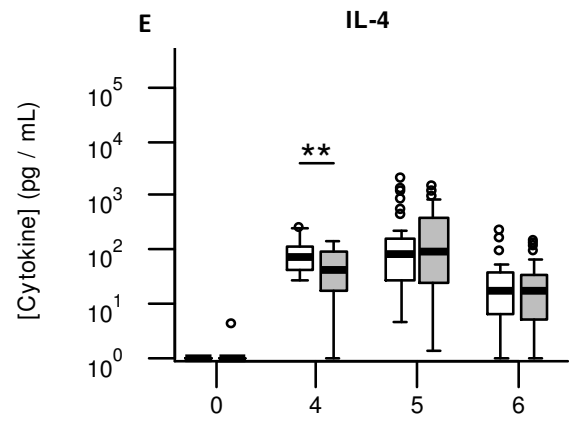

Polarization day s

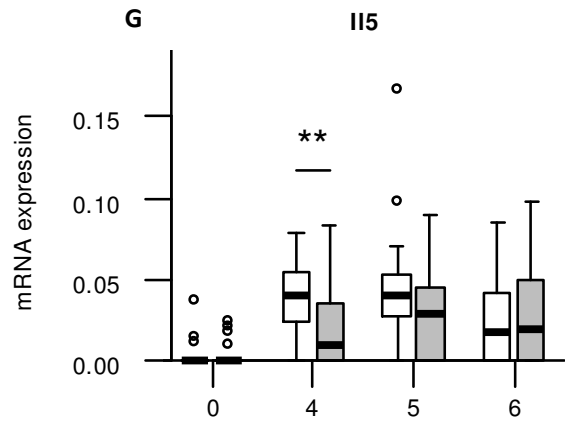

Polarization days

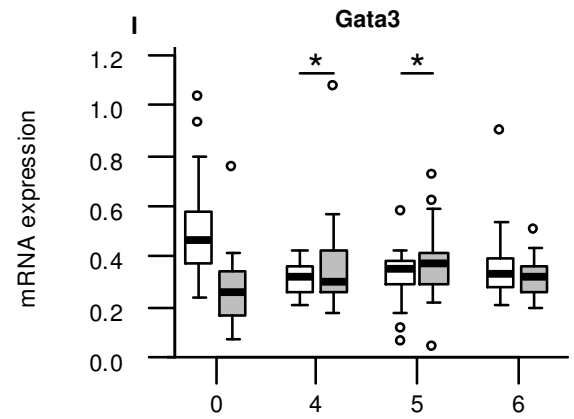

Polarization days

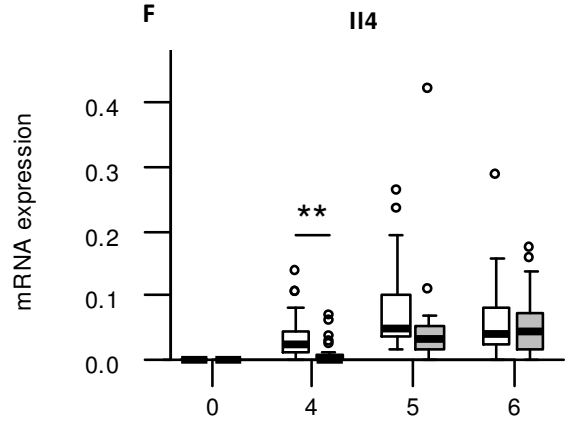

Polarization days

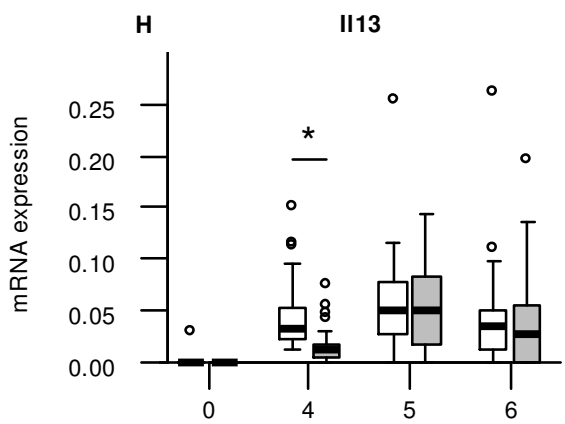

Polarization days

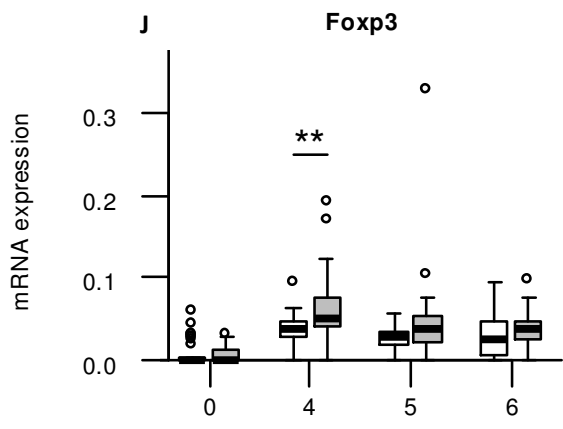

Polarization days

Figure 7. Perinatal fish oil diet (FOD) reduces extracellular cytokine levels of ex vivo Th1-polarizing $\mathrm{CD}^{+} \mathrm{T}$ cells. Extracellular levels of IFN- $\gamma$ (A), IL-13 (B), IL-10 (C), IL-5 (D) and IL-4 (E), and mRNA expression of $/ / 4(F), / 15(\mathrm{G}), / 113(\mathrm{H})$, Gata3 (I), and Foxp3 (J) were measured at 0, 4, 5 and 6 days after incubation of naïve $C D 4^{+} T$ cells in presence of $\alpha-C D 3, \alpha-C D 28, I L-2$ and IL-12. Splenic naïve CD4 ${ }^{+} T$ cells were isolated at the end of lactation from offspring after dams received a control diet (CD) or FOD during gestation and lactation. Data are presented as boxplots. Statistical analysis by linear mixedeffects model. $n=32-35$ vs. $26-27$ ( $A-E ; C D$ vs. FOD) or $n=33-34$ vs. $25-27$ ( $F-J ; C D$ vs. FOD). ${ }^{*} p<0.05$, ${ }^{* *} p<0.01, * * * p<0.001$ for corresponding diet*day interaction. 
In the ex vivo polarization assay, Th2 polarization increased extracellular levels of IL-5, IL13, IFN- $\gamma$ and IL-10, with the smallest elevation for IFN- $\gamma$. Furthermore, Th2 polarization increased gene expression of cytokine genes $/ 14,1 / 5,1 / 13$ and /fng, and of transcription factors Gata3 and Tbx21, but not of $/ / 10$ and Foxp3. Th2 polarization did not affect any Th17 marker (i.e., extracellular levels of IL-17a, and mRNA expression of II17a and Rorc). Comparing polarization responses between cells from $C D$ offspring and cells from FOD offspring showed that similar to Th1 polarization, the perinatal FOD also affected Th2 polarization-induced responses, again with most consistent effects on extracellular cytokine levels, with extracellular levels of IL-5, IL-13 and IL-10 being significantly lower in the FOD group than in the $\mathrm{CD}$ group (Figure $8 \mathrm{a}-\mathrm{C}$ ). IFN- $\gamma$ levels, however, did not differ between FOD and CD (Figure S3). On the level of gene expression, the perinatal FOD only affected expression of //13 and Gata3, for which mRNA levels were significantly higher in the FOD group than the $C D$ group on polarization day 6 or on polarization day 4 , respectively (Figure $8 \mathrm{~d}$-e). Expression levels of $/ 14,1 / 5$, Ifng, II10, Tbx21 and Foxp3 were not affected during Th2 polarization by the perinatal FOD (Figure S3). Altogether, these results show that a perinatal FOD reduced extracellular cytokine levels of ex vivo Th1- and Th2-polarizing offspring CD4 ${ }^{+}$ T cells. 


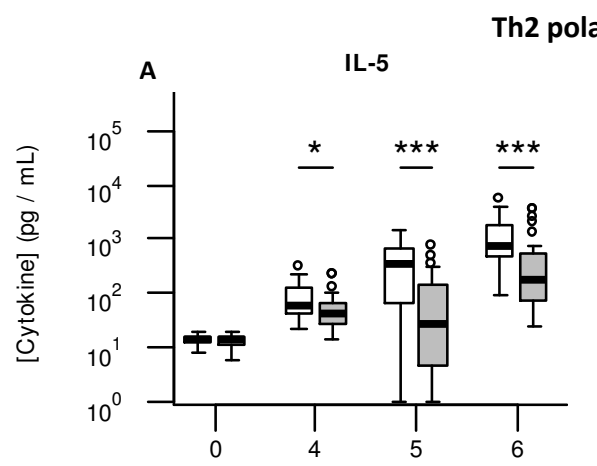

Polarization days

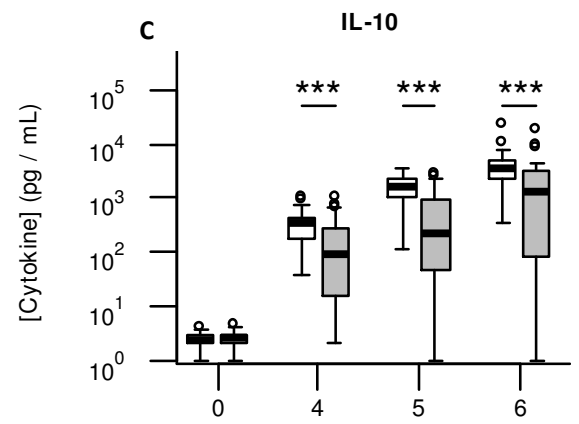

Polarization day s

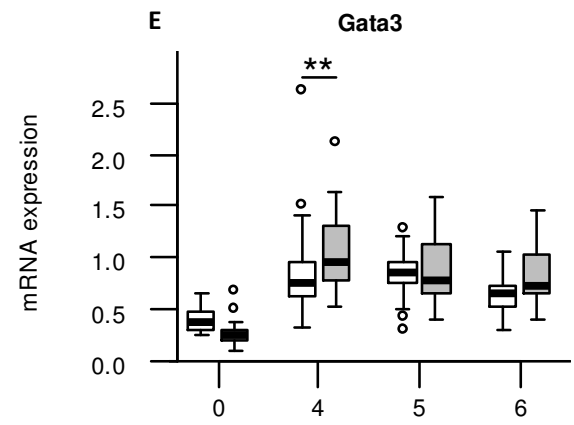

Polarization day s

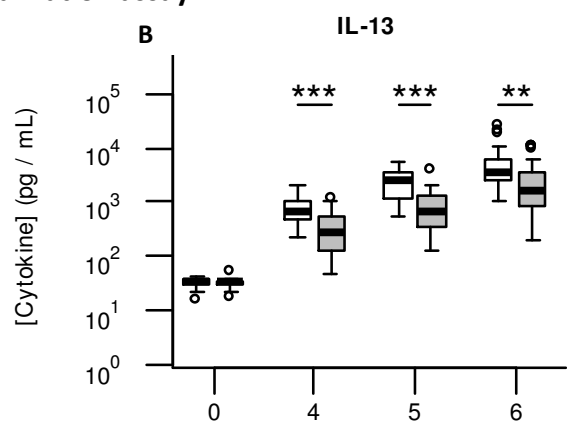

Polarization days

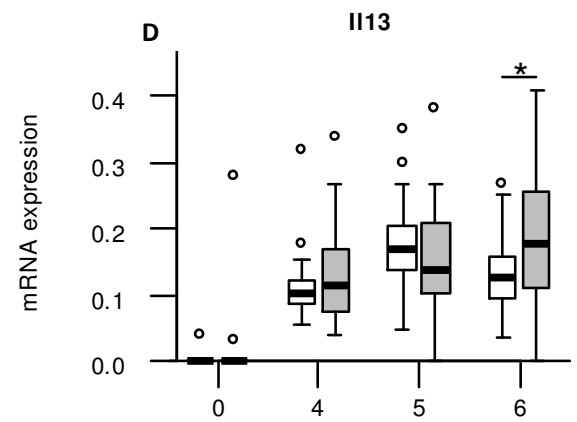

Polarization day s $\square$ Control diet

Fish oil diet

Figure 8. Perinatal fish oil diet (FOD) reduces extracellular cytokine levels of ex vivo Th2-polarizing $\mathrm{CD}^{+} \mathrm{T}$ cells. Extracellular levels of IL-5 (A), IL-13 (B) and IL-10 (C), and mRNA expression of III3 (D) and Gata3 (E) were measured at 0, 4, 5 and 6 days after incubation of naïve $\mathrm{CD}^{+}{ }^{+} \mathrm{T}$ cells in presence of $\alpha-C D 3, \alpha-C D 28$, IL-2 and IL-4. Splenic naïve $C D 4^{+} T$ cells were isolated at the end of lactation from offspring after dams received a control diet or FOD during gestation and lactation. Data are presented as boxplots. Statistical analysis by linear mixed-effects model. $n=34$ vs. 27 ( $A-C$; CD vs. FOD) or $\mathrm{n}=23-30$ vs. 21 ( $\mathrm{D}-\mathrm{E}$; CD vs. FOD). ${ }^{*} \mathrm{p}<0.05,{ }^{* *} \mathrm{p}<0.01,{ }^{* * *} \mathrm{p}<0.001$ for corresponding diet*day interaction. 


\section{Discussion}

Previous research has shown that maternal supplementation with $\mathrm{n}-3$ PUFAs during pregnancy is associated with reductions of asthmatic features in offspring [9-11]. The underlying mechanisms, however, are unclear. Since a skewed polarization of $\mathrm{CD}^{+} \mathrm{T}$ cells is involved in asthmatic development, we studied the effect of a perinatal n-3 PUFA-rich FOD on ex vivo polarization of $\mathrm{CD}^{+} \mathrm{T}$ cells in mouse offspring. In this study, we showed that a perinatal FOD reduced extracellular cytokine levels of both Th1- and Th2-polarizing CD4 ${ }^{+}$ $T$ cells. Furthermore, we found that a perinatal FOD induced not only anemia in both dams and offspring, but also microvesicular steatosis in offspring liver.

As expected, the perinatal FOD altered the maternal plasma fatty acid profile, with higher plasma levels of EPA, DHA and total n-3 PUFAs; lower plasma levels of total n-6 PUFAs; and similar levels of total MUFAs in FOD dams compared with CD dams. On the other hand, total PUFAs and total SFAs were designed to be equal in both diets, but resulted in lower plasma levels in FOD dams compared with CD dams. For total SFAs, this discrepancy between dietary levels and plasma phospholipid levels is most likely a result of metabolism, since SFAs are not only taken up from the diet, but also synthesized de novo. PUFAs, in contrast, cannot be synthesized de novo by mammals, so total plasma PUFA concentrations were expected to reflect dietary intake levels. However, it is known from literature that for $\alpha$ linolenic acid, which is the main n-3 PUFA in the CD and FOD, plasma phospholipid levels do not correlate with dietary intake levels [26]. As a result, the difference in total n-3 PUFA plasma levels between FOD and CD dams was smaller than the difference in total n-6 PUFA plasma levels, and as a consequence, total PUFA plasma levels were lower for FOD dams than for $C D$ dams. Altogether, these findings indicate that the perinatal FOD had the intended effect on maternal plasma FA levels.

The perinatal FOD unexpectedly induced anemia in both dams and offspring. Although this observation was unanticipated, studies have previously indicated a potential link between diets high in n-3 PUFAs and anemia. Both Miret et al. and Rodriguez et al. demonstrated that feeding rats an FOD induced oxidative stress in erythrocytes $[27,28]$, which in turn increases erythrocyte aging and removal by macrophages $[29,30]$. For the studies by Miret et al. and Rodriguez et al., erythrocyte counts were not affected and anemia was not observed, since the increased aging and removal of erythrocytes was compensated by an increased erythropoiesis, as indicated by elevated reticulocyte counts, decreased iron stores in liver and spleen, and increased iron absorption $[27,28]$. This corresponds with our study, since FOD dams did show extramedular hematopoiesis, but no external signs of anemia, suggesting that any potential FOD-induced increase in erythrocyte turnover was indeed compensated by enhanced erythropoiesis. However, pups from FOD dams did show 
anemic symptoms, such as lethargy and reduced bodyweight. We hypothesize that the exposure to the high n-3 PUFA levels of these pups occurred during such a crucial developmental period, that the suggested increased erythrocyte turnover could not be compensated by accelerated erythropoiesis and hence led to observable anemia.

Next to anemia, the perinatal FOD also induced microvesicular steatosis in livers from FOD offspring. Microvesicular steatosis is the accumulation of small lipid droplets in the cytoplasm, leaving the nucleus in the center of the cell [31]. This is in contrast with macrovesicular steatosis, which is characterized by the cytoplasm being filled with larger lipid vacuoles and displacement of the nucleus to the periphery of the cell. Macrovesicular steatosis is a more common form of lipid accumulation and can be observed, for instance, in cases of obesity, insulin resistance and alcoholism. Microvesicular steatosis, on the other hand, is rare and is associated with intoxications and diseases which involve an impairment of mitochondrial $\beta$-oxidation [32]. The observation of hepatic lipid accumulation in offspring from FOD dams in our study was unexpected, since n-3 PUFAs are known to reduce hepatic lipid content through the reduction of lipogenesis and elevation of fatty acid oxidation and VLDL secretion [33]. Possibly, it is due to the perinatal period during which pups were exposed to the FOD which led to the observed hepatic lipid accumulation. During this crucial developmental period, pups may have been more vulnerable to the hepatic effects of the perinatal FOD than the adult animals that are usually used in experiments. Nevertheless, presence of the characteristic microvesicular structure in our study, in contrast to the macrovesicular structure, may be explained by the high n-3 PUFA content of the FOD. Previous studies have shown that in diabetic patients, increased fat droplet size in fatty livers was associated with reduced hepatic content of EPA and vice versa [34]. Similarly, a mouse model for hepatic microvesicular steatosis had an identical hepatic n-6:n-3 PUFA ratio as lean mice, whereas mice with hepatic marovesicular steatosis had a higher hepatic n-6:n-3 PUFA ratio [35]. Moreover, n-3 PUFA supplementation of $o b / o b$ mice has previously been shown to convert macrovesicular steatosis into microvesicular steatosis [36].

Using an ex vivo polarization assay, we showed that a perinatal FOD affected ex vivo Th1 and Th2 polarization of naïve CD4 ${ }^{+} \mathrm{T}$ cells. Most prominently, the perinatal FOD significantly reduced extracellular levels of IL-4, IL-5, IL-13, IFN- $\gamma$ and IL-10 during Th1 polarization, and IL-5, IL-13 and IL-10 during Th2 polarization. The perinatal FOD affected mRNA expression of Th-specific cytokines and transcription factors as well, but these changes were less consistent than the effects observed for extracellular cytokine levels. Although mRNA and protein levels do not necessarily have to correlate, as previously indicated [37], the discrepancy between FOD-induced effects on gene and protein expression may suggest that the decreased extracellular cytokine levels observed for FOD offspring were a result of a reduced number of cytokine-secreting cells, rather than a reduced cytokine secretion per 
cell. Indeed, previous studies have shown that feeding mice an n-3 PUFA-rich diet reduces the ex vivo proliferative capacity of $\mathrm{CD}^{+} \mathrm{T}$ cells [38-42]. Next to this, ex vivo proliferation of $\mathrm{CD}^{+} \mathrm{T}$ cells can be reduced by hypoxia as well $[43,44]$, suggesting that the observed decrease in extracellular cytokines levels during ex vivo polarization of $\mathrm{CD} 4^{+} \mathrm{T}$ cells may have been a consequence of the perinatal FOD-induced anemia, rather than being a direct effect of the perinatal FOD.

For all experimental outcomes measured in offspring animals, statistical analyses were adjusted for offspring sex. This approach was determined a priori, since sex hormones are known to influence immune responses [45]. In the current study, however, none of the performed analyses showed a significant effect of sex. This lack of sex effect may be due to the relatively young age at which outcomes were assessed in offspring animals. At this prepubertal age, sex hormone levels are still relatively similar for male and female mice [46]. Thus, hormonal differences between male and female offspring in the current study may not have been sufficiently developed to induce sex differences.

A limitation of our study is that the hypothesized decrease in $\mathrm{CD}^{+} \mathrm{T}$ cell proliferation could not be confirmed, since cell proliferation was not assessed. As a consequence, it remains unclear whether the perinatal FOD-induced decrease in cytokine levels was related to a lower cytokine production per cell, to lower cell numbers, or a combination of both. Next to this, outcomes related to the polarization assay were assessed only at the whole-sample level. Although these outcomes provide valuable insights in the influence of a perinatal FOD on ex vivo $\mathrm{CD}^{+}{ }^{+}$cell polarization, it remains unclear what changes occurred the singlecell level. For instance, Th1-polarizing cells were found to produce not only Th1 cytokine IFN- $\gamma$, but also Th2-cytokines IL-4, IL-5 and IL-13, and Treg cytokine IL-10. Additionally measuring these cytokines intracellularly at the single-cell level using flow cytometry, would have shown to what extent these cytokines were produced by distinct cell populations.

A particular strength of this study is the use of linear mixed-effects models for analysis of outcomes assessed in offspring. When studying the effect of a prenatal treatment, randomization can only be applied to the dam, whereas outcomes are measured in individual offspring animals. Since animals from the same litter are more alike compared with animals from different litters, this introduces the so-called litter effects. Ignoring these litter effects by analyzing data with standard methods such as t-test or linear regression may lead to increased false positive rates or low power [47]. Using linear mixed-effects models, in contrast, allows to take into account the multilevel structure of the experiment, while at the same time being able to adjust for covariates such as offspring sex. 
For future studies, different approaches to both the animal model and the polarization assay may be applied. For the animal model, BALB/CByJ mice were used, since animals from this strain have been shown to develop a Th2-dominant immune response [48-50]. This mimics the Th2-dominant immune response that characterizes patients with asthma and other atopic conditions. It would, however, be interesting to study the effect of a perinatal FOD in other strains as well, such as the more Th1-dominant C57BL/6 strain. For the polarization assay, $C D 4^{+} T$ cells were activated by incubating them ex vivo with anti-CD3 and anti-CD28. While this induction with exogenous molecules has been widely applied to study $\mathrm{CD} 4^{+} \mathrm{T}$ cell functions, it differs from the in vivo situation, in which naïve $\mathrm{CD} 4^{+} \mathrm{T}$ cells are activated by endogenous APCs. Future studies may therefore mimic the in vivo situation more by activating naïve $\mathrm{CD} 4^{+} \mathrm{T}$ cells ex vivo with endogenous APCs. Alternatively, future studies may examine the effect of a perinatal FOD on $\mathrm{CD}^{+} \mathrm{T}$ cell polarization in vivo, for example by inducing an allergic response in offspring animals through sensitization and challenge with an allergen, rather than isolating naïve $\mathrm{CD}^{+} \mathrm{T}$ cells and polarizing them ex vivo.

The effects observed in our study may be considered as both beneficial and adverse. As a beneficial effect, the perinatal FOD reduced extracellular cytokine levels of Th1- and Th2polarizing $\mathrm{CD}^{+} \mathrm{T}$ cells. This reduced cytokine response upon polarization may, in part, explain the positive effects of prenatal supplementation with fish oil on asthma-related outcomes that were previously reported in clinical studies [9-11]. On the other hand, the perinatal FOD in our study induced anemia and microvesicular hepatic steatosis. These adverse effects may be the result of the relatively high dose of $n-3$ PUFAs present in the FOD diet, since the daily consumption of n-3 PUFAs per kg of bodyweight was approximately 10 times higher for the dams in our study compared with the pregnant women in the COPSAC study [9]. Thus, our findings stress the need to carefully consider dosages for future studies, both in vivo and clinical. The Inuit for instance, which have a high intake of $n-3$ PUFAs, are known to have a high prevalence of unexplained anemia (i.e. anemia which is not related to a micronutrient deficiency) [51], suggesting that a high intake of n-3 PUFAs could potentially lead to adverse effects in humans as well.

In summary, we showed that a perinatal FOD decreased extracellular cytokine levels of CD4 ${ }^{+}$ T cells during ex vivo Th1 and Th2 polarization. Furthermore, the perinatal FOD induced anemia in both dams and offspring, and microvesicular steatosis in offspring liver. More research is needed to unravel the molecular mechanisms that underlie these effects and to find out what share they have in the beneficial effects of maternal $n-3$ PUFA supplementation on offspring asthma risk. 


\section{Acknowledgement}

The authors would like to thank Marie-José Drittij for technical assistance with gene expression analysis, and Richard Frijnts, Rik Tinnemans and Nicole Bitsch for animal handling and care. 


\section{References}

1. Eder, W.; Ege, M.J.; von Mutius, E. The asthma epidemic. New England Journal of Medicine 2006, 355, 2226-2235.

2. Centers for Disease Control and Prevention, Asthma. Available online: https://www.cdc.gov/asthma/data-visualizations/default.htm (accessed on 23-06-2021).

3. Asthma UK, Asthma prevalence. Available online: https://public.tableau.com/app/profile/asthmauk/viz/Asthmaprevalence/Asthmaprevalen ce (accessed on 23-06-2021).

4. Eurostat Data Browser, Persons reporting a chronic disease. Available online: http://appsso.eurostat.ec.europa.eu/nui/show.do?dataset=hlth_ehis_cd1e\&lang=en (accessed on 23-06-2021).

5. Black, P.; Sharpe, S. Dietary fat and asthma: is there a connection? European Respiratory Journal 1997, 10, 6-12.

6. Lumia, M.; Luukkainen, P.; Tapanainen, H.; Kaila, M.; Erkkola, M.; Uusitalo, L.; Niinistö, S.; Kenward, M.G.; Ilonen, J.; Simell, O. Dietary fatty acid composition during pregnancy and the risk of asthma in the offspring. Pediatr Allergy Immu 2011, 22, 827-835.

7. Miyake, Y.; Sasaki, S.; Tanaka, K.; Ohfuji, S.; Hirota, Y. Maternal fat consumption during pregnancy and risk of wheeze and eczema in Japanese infants aged 16-24 months: the Osaka Maternal and Child Health Study. Thorax 2009, 64, 815-821.

8. Pike, K.C.; Calder, P.C.; Inskip, H.M.; Robinson, S.M.; Roberts, G.C.; Cooper, C.; Godfrey, K.M.; Lucas, J.S. Maternal plasma phosphatidylcholine fatty acids and atopy and wheeze in the offspring at age of 6 years. Clinical and Developmental Immunology 2012, 2012.

9. Bisgaard, H.; Stokholm, J.; Chawes, B.L.; Vissing, N.H.; Bjarnadóttir, E.; Schoos, A.-M.M.; Wolsk, H.M.; Pedersen, T.M.; Vinding, R.K.; Thorsteinsdóttir, S. Fish oil-derived fatty acids in pregnancy and wheeze and asthma in offspring. New England Journal of Medicine 2016, $375,2530-2539$.

10. Hansen, S.; Strøm, M.; Maslova, E.; Dahl, R.; Hoffmann, H.J.; Rytter, D.; Bech, B.H.; Henriksen, T.B.; Granström, C.; Halldorsson, T.I. Fish oil supplementation during pregnancy and allergic respiratory disease in the adult offspring. J Allergy Clin Immun 2017, 139, 104111. e104.

11. Olsen, S.F.; Østerdal, M.L.; Salvig, J.D.; Mortensen, L.M.; Rytter, D.; Secher, N.J.; Henriksen, T.B. Fish oil intake compared with olive oil intake in late pregnancy and asthma in the offspring: 16 y of registry-based follow-up from a randomized controlled trial. The American journal of clinical nutrition 2008, 88, 167-175.

12. Yoo, Y.; Perzanowski, M.S. Allergic sensitization and the environment: latest update. Current allergy and asthma reports 2014, 14, 465.

13. Holt, P.; Naspitz, C.; Warner, J.O. Early immunological influences. Prevention of Allergy and Allergic Asthma 2004, 84, 102-127.

14. Rodriguez, R.M.; Lopez-Larrea, C.; Suarez-Alvarez, B. Epigenetic dynamics during CD4+ T cells lineage commitment. The international journal of biochemistry \& cell biology 2015, 67, 75-85. 
15. Borish, L.; Aarons, A.; Rumbyrt, J.; Cvietusa, P.; Negri, J.; Wenzel, S. Interleukin-10 regulation in normal subjects and patients with asthma. J Allergy Clin Immun 1996, 97, 1288-1296.

16. Kim, C.K.; Kim, S.W.; Park, C.S.; Kim, B.I.; Kang, H.; Koh, Y.Y. Bronchoalveolar lavage cytokine profiles in acute asthma and acute bronchiolitis. J Allergy Clin Immun 2003, 112, 64-71.

17. Molet, S.; Hamid, Q.; Davoineb, F.; Nutku, E.; Tahaa, R.; Pagé, N.; Olivenstein, R.; Elias, J.; Chakir, J. IL-17 is increased in asthmatic airways and induces human bronchial fibroblasts to produce cytokines. J Allergy Clin Immun 2001, 108, 430-438.

18. Shahid, S.K.; Kharitonov, S.A.; Wilson, N.M.; Bush, A.; Barnes, P.J. Increased interleukin-4 and decreased interferon $-\gamma$ in exhaled breath condensate of children with asthma. American journal of respiratory and critical care medicine 2002, 165, 1290-1293.

19. Van Der Lee, S.; Boot, L.M. Spontaneous pseudopregnancy in mice. II. Acta Physiol Pharmacol Neerl 1956, 5, 213-215.

20. Whitten, W.K. Modification of the oestrous cycle of the mouse by external stimuli associated with the male. Journal of Endocrinology 1956, 13, 399-404.

21. Ruijter, J.; Ramakers, C.; Hoogaars, W.; Karlen, Y.; Bakker, O.; Van den Hoff, M.; Moorman, A. Amplification efficiency: linking baseline and bias in the analysis of quantitative PCR data. Nucleic acids research 2009, 37, e45-e45.

22. Schött, H.-F.; Konings, M.C.; Schrauwen-Hinderling, V.B.; Mensink, R.P.; Plat, J. A Validated Method for Quantification of Fatty Acids Incorporated in Human Plasma Phospholipids by Gas Chromatography-Triple Quadrupole Mass Spectrometry. ACS omega 2021, 6, 11291137.

23. Maner, B.S.; Moosavi, L. Mean corpuscular volume (MCV). 2019.

24. Council, N.R. Nutrient requirements of laboratory animals: 1995. 1995.

25. Kowdley, K.V.; Gochanour, E.M.; Sundaram, V.; Shah, R.A.; Handa, P. Hepcidin Signaling in Health and Disease: Ironing Out the Details. Hepatology communications 2021.

26. Astorg, P.; Bertrais, S.; Laporte, F.; Arnault, N.; Estaquio, C.; Galan, P.; Favier, A.; Hercberg, S. Plasma n-6 and n-3 polyunsaturated fatty acids as biomarkers of their dietary intakes: a cross-sectional study within a cohort of middle-aged French men and women. European journal of clinical nutrition 2008, 62, 1155-1161.

27. Miret, S.; Saiz, M.; Mitjavila, M. Effects of fish oil-and olive oil-rich diets on iron metabolism and oxidative stress in the rat. British journal of nutrition 2003, 89, 11-18.

28. Rodríguez, M.C.; Sáiz, M.P.; Muntané, J.; Mitjavila, M.T. Fatty acid composition of erythrocyte membranes affects iron absorption in rats. The Journal of nutrition 1996, 126, 3109-3117.

29. Pradhan, D.; Weiser, M.; Lumley-Sapanski, K.; Frazier, D.; Kemper, S.; Williamson, P.; Schlegel, R.A. Peroxidation-induced perturbations of erythrocyte lipid organization. Biochimica et Biophysica Acta (BBA)-Biomembranes 1990, 1023, 398-404.

30. Signorini, C.; Ferrali, M.; Ciccoli, L.; Sugherini, L.; Magnani, A.; Comporti, M. Iron release, membrane protein oxidation and erythrocyte ageing. FEBS letters 1995, 362, 165-170.

31. Fromenty, B.; Berson, A.; Pessayre, D. Microvesicular steatosis and steatohepatitis: role of mitochondrial dysfunction and lipid peroxidation. Journal of hepatology 1997, 26, 13-22.

32. Fromenty, B.; Pessayre, D. Inhibition of mitochondrial beta-oxidation as a mechanism of hepatotoxicity. Pharmacology \& therapeutics 1995, 67, 101-154. 
33. El-Badry, A.M.; Graf, R.; Clavien, P.-A. Omega 3-Omega 6: What is right for the liver? Journal of hepatology 2007, 47, 718-725.

34. Singer, P.; Honigmann, G.; Schliack, V. Decrease of eicosapentaenoic acid in fatty liver of diabetic subjects. Prostaglandins and medicine 1980, 5, 183-200.

35. El-Badry, A.M.; Moritz, W.; Contaldo, C.; Tian, Y.; Graf, R.; Clavien, P.A. Prevention of reperfusion injury and microcirculatory failure in macrosteatotic mouse liver by omega-3 fatty acids. Hepatology 2007, 45, 855-863.

36. Alwayn, I.P.; Andersson, C.; Zauscher, B.; Gura, K.; Nosé, V.; Puder, M. Omega-3 fatty acids improve hepatic steatosis in a murine model: potential implications for the marginal steatotic liver donor. Transplantation 2005, 79, 606-608.

37. Godschalk, R.; Remels, A.; Hoogendoorn, C.; van Benthem, J.; Luijten, M.; Duale, N.; Brunborg, G.; Olsen, A.-K.; Bouwman, F.G.; Munnia, A.; et al. Paternal Exposure to Environmental Chemical Stress Affects Male Offspring's Hepatic Mitochondria. Toxicol Sci 2017, 162, 241-250, doi:10.1093/toxsci/kfx246.

38. Brix, S.; Lund, P.; Kjaer, T.M.; Straarup, E.M.; Hellgren, L.I.; Frøkiær, H. CD4+ T-cell activation is differentially modulated by bacteria-primed dendritic cells, but is generally downregulated by $\mathrm{n}-3$ polyunsaturated fatty acids. Immunology 2010, 129, 338-350.

39. Chapkin, R.; Arrington, J.; Apanasovich, T.; Carroll, R.; McMurray, D. Dietary n-3 PUFA affect TcR-mediated activation of purified murine T cells and accessory cell function in co-cultures. Clinical \& Experimental Immunology 2002, 130, 12-18.

40. Hou, T.Y.; Barhoumi, R.; Fan, Y.-Y.; Rivera, G.M.; Hannoush, R.N.; McMurray, D.N.; Chapkin, R.S. n-3 polyunsaturated fatty acids suppress CD4+ T cell proliferation by altering phosphatidylinositol-(4, 5)-bisphosphate [PI $(4,5) \mathrm{P} 2]$ organization. Biochimica et Biophysica Acta (BBA)-Biomembranes 2016, 1858, 85-96.

41. Kim, W.; Fan, Y.-Y.; Barhoumi, R.; Smith, R.; McMurray, D.N.; Chapkin, R.S. n-3 polyunsaturated fatty acids suppress the localization and activation of signaling proteins at the immunological synapse in murine CD4+ T cells by affecting lipid raft formation. The Journal of Immunology 2008, 181, 6236-6243.

42. Ly, L.H.; Smith, R.; Switzer, K.C.; Chapkin, R.S.; McMurray, D.N. Dietary eicosapentaenoic acid modulates CTLA-4 expression in murine CD4+ T-cells. Prostaglandins, leukotrienes and essential fatty acids 2006, 74, 29-37.

43. Gaber, T.; Tran, C.L.; Schellmann, S.; Hahne, M.; Strehl, C.; Hoff, P.; Radbruch, A.; Burmester, G.R.; Buttgereit, F. Pathophysiological hypoxia affects the redox state and IL-2 signalling of human CD4+ T cells and concomitantly impairs survival and proliferation. European journal of immunology 2013, 43, 1588-1597.

44. Westendorf, A.M.; Skibbe, K.; Adamczyk, A.; Buer, J.; Geffers, R.; Hansen, W.; Pastille, E.; Jendrossek, V. Hypoxia enhances immunosuppression by inhibiting CD4+ effector T cell function and promoting Treg activity. Cellular Physiology and Biochemistry 2017, 41, 12711284.

45. Taneja, V. Sex hormones determine immune response. Frontiers in immunology 2018, 9, 1931.

46. Bell, M.R. Comparing postnatal development of gonadal hormones and associated social behaviors in rats, mice, and humans. Endocrinology 2018, 159, 2596-2613. 
47. Lazic, S.E.; Essioux, L. Improving basic and translational science by accounting for litter-tolitter variation in animal models. BMC neuroscience 2013, 14, 1-11.

48. Mills, C.D.; Kincaid, K.; Alt, J.M.; Heilman, M.J.; Hill, A.M. M-1/M-2 macrophages and the Th1/Th2 paradigm. The Journal of immunology 2000, 164, 6166-6173.

49. Schulte, S.; Sukhova, G.K.; Libby, P. Genetically programmed biases in Th1 and Th2 immune responses modulate atherogenesis. The American journal of pathology 2008, 172, 1500 1508.

50. Watanabe, H.; Numata, K.; Ito, T.; Takagi, K.; Matsukawa, A. Innate immune response in Th1and Th2-dominant mouse strains. Shock 2004, 22, 460-466.

51. Jamieson, J.A.; Weiler, H.A.; Kuhnlein, H.V.; Egeland, G.M. Prevalence of unexplained anaemia in Inuit men and Inuit post-menopausal women in northern Labrador: international polar year Inuit health survey. Canadian Journal of Public Health 2016, 107, e81-e87. 


\section{Supplement}

Table S1. Detailed formulas for control diet (CD) and fish oil diet (FOD).

\begin{tabular}{|c|c|c|}
\hline & CD (g/kg) & FOD $(\mathrm{g} / \mathrm{kg})$ \\
\hline \multicolumn{3}{|l|}{ Protein } \\
\hline Casein & 261 & 261 \\
\hline L-cysteine & 4 & 4 \\
\hline \multicolumn{3}{|l|}{ Carbohydrate } \\
\hline Corn starch & 354 & 354 \\
\hline Maltodextrin 10 & 110 & 110 \\
\hline Dextrose & 150 & 150 \\
\hline \multicolumn{3}{|l|}{ Fiber } \\
\hline Cellulose, BW200 & 75 & 75 \\
\hline Inulin & 25 & 25 \\
\hline \multicolumn{3}{|l|}{ Fat } \\
\hline Soybean oil & 54 & - \\
\hline Menhaden oil & - & 15 \\
\hline Flaxseed oil & - & 40 \\
\hline Cholesterol & $0.08^{1}$ & $0.08^{2}$ \\
\hline \multicolumn{3}{|l|}{ Minerals } \\
\hline Mineral Mix S10026 & 10 & 10 \\
\hline Dicalcium Phosphate & 13 & 13 \\
\hline Calcium carbonate & 5.5 & 5.5 \\
\hline Potassium citrate & 16.5 & 16.5 \\
\hline \multicolumn{3}{|l|}{ Vitamins } \\
\hline Vitamin mix V10001 & 10 & 10 \\
\hline Choline bitartrate & 20 & 20 \\
\hline \multicolumn{3}{|l|}{ Antioxidant } \\
\hline Tert-butylhydroquinone & $2.7 \mathrm{mg} / \mathrm{kg}$ & $2.7 \mathrm{mg} / \mathrm{kg}$ \\
\hline
\end{tabular}

${ }^{1}$ Added to match FOD; ${ }^{2}$ Naturally occurring in menhaden oil 

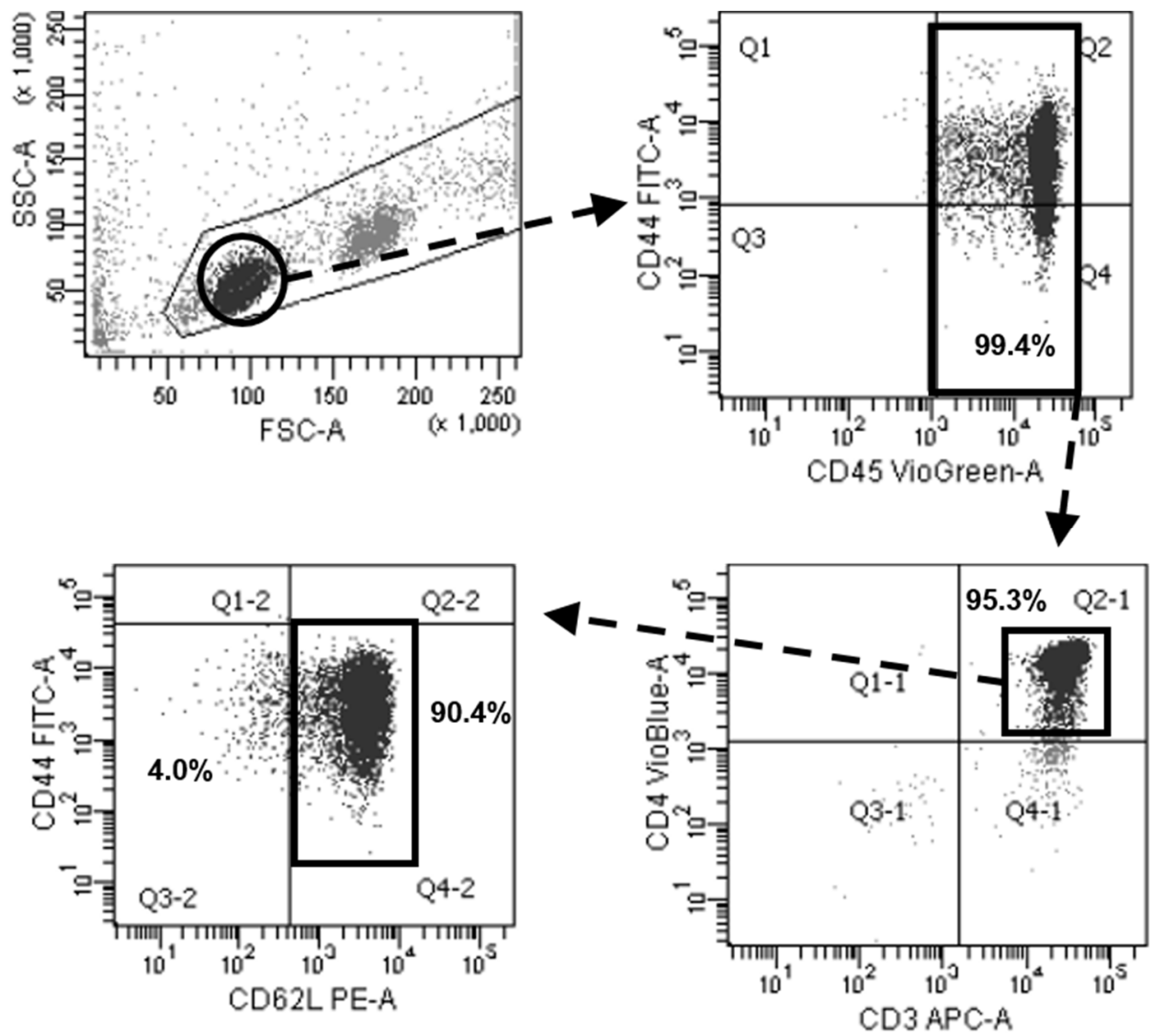

Figure S1. Purity of naïve $\mathrm{CD}^{+} \mathrm{T}$ cells after enrichment from spleen single-cell suspension. Cells were gated on singlet lymphocytes in $\mathrm{FSC} / \mathrm{SSC}$ and set as $100 \%$. CD4 ${ }^{+} \mathrm{T}$ cells were gated on $\mathrm{CD}_{4} 5^{+}(\mathrm{Q} 2+\mathrm{Q} 4)$ and $\mathrm{CD3}^{+} / \mathrm{CD4}^{+}(\mathrm{Q} 2-1)$, and subsequently distinguished between naïve (CD62 $\left.\mathrm{L}^{\text {high }} \mathrm{CD} 44^{\text {low; }} \mathrm{Q} 4-2\right)$, effector (CD62 $L^{\text {low }} C D 44^{\text {low; }}$ Q3-2), central memory (CD62 $L^{\text {low }} C D 44^{\text {high }}$; Q1-2), and effector memory (CD62L high CD44high; Q2-2). 
Table S2. Flow cytometry antibodies.

\begin{tabular}{lll}
\hline Antibody & Manufacturer & Catalog number \\
\hline CD62-PE & Miltenyi Biotec & $130-112-836$ \\
CD4-VioBlue & Miltenyi Biotec & $130-118-696$ \\
CD3-APC & Miltenyi Biotec & $130-122-943$ \\
CD44-VioBright FITC & Miltenyi Biotec & $130-120-287$ \\
CD45-VioGreen & Miltenyi Biotec & $130-110-803$ \\
\hline
\end{tabular}

Table S3. Cell culture antibodies and cytokines.

\begin{tabular}{lll}
\hline Antibody/cytokine & Manufacturer & Catalog number \\
\hline Dynabeads Mouse T-activator CD3/CD28 & Thermo Fisher Scientific & $11452 \mathrm{D}$ \\
IL-2, mouse & Miltenyi Biotec & $130-120-330$ \\
IL-12, mouse & Miltenyi Biotec & $130-096-708$ \\
IL-4, mouse & Miltenyi Biotec & $130-097-757$ \\
\hline
\end{tabular}

Table S4. Real-time quantitative PCR primer sequences (5'-3').

\begin{tabular}{lll}
\hline Gene & Forward primer & Reverse primer \\
\hline Ex vivo polarization & \\
Ifng & ATGAACGCTACACACTGCATC & CCATCCTTTTGCCAGTTCCTC \\
Tbx21 & AGCAAGGACGGCGAATGTT & GGGTGGACATATAAGCGGTTC \\
II4 & ATCATCGGCATTTTGAACGAGG & TGCAGCTCCATGAGAACACTA \\
II5 & CTCTGTTGACAAGCAATGAGACG & TCTTCAGTATGTCTAGCCCCTG \\
$\| 13$ & TGAGCAACATCACACAAGACC & GGCCTTGCGGTTACAGAGG \\
Gata3 & TCCTTGCTACTCAGGTGATCG & TGACCACACTGCACACTGAT \\
II10 & AGCCTTATCGGAAATGATCCAGT & GGCCTTGTAGACACCTTGGT \\
Foxp3 & CCCATCCCCAGGAGTCTTG & ACCATGACTAGGGGCACTGTA \\
II17a & TTTAACTCCCTTGGCGCAAAA & CTTTCCCTCCGCATTGACAC \\
Rorc & GACCCACACCTCACAAATTGA & AGTAGGCCACATTACACTGCT \\
Cd3e & ATGCGGTGGAACACTTTCTGG & GCACGTCAACTCTACACTGGT \\
Actb & ATGGAGGGGATACAGCCC & TTCTTTGCAGCTCCTTCGTT \\
& & \\
Offspring liver & \\
Hamp & GCTGCCTGTCTCCTGCTTCT & GCTCTGTAGTCTGTCTCATCTGTTGAT \\
Gapdh & CATTGTGGAAGGGCTCATGA & GCCCCACGGCCATCA \\
Hprt & TCAGTCAACGGGGGACATAAA & GGGGCTGTACTGCTTAACCAG \\
\hline
\end{tabular}


Table S5. Individual concentrations of free phospholipid fatty acids measured in dam plasma at the end of lactation (on postnatal day 21) after dams received a control diet (CD) or fish oil diet (FOD) during gestation and lactation. Statistical analysis by unpaired, two-tailed t-test, $n=6$ per group. SFA, saturated fatty acids; MUFA, monounsaturated fatty acids; PUFA, polyunsaturated fatty acids; $95 \% \mathrm{Cl}$, 95\% confidence interval.

\begin{tabular}{|c|c|c|c|}
\hline & $\begin{array}{l}\mathrm{CD}(\mathrm{mM}) \\
\text { mean }(95 \% \mathrm{Cl})\end{array}$ & $\begin{array}{l}\text { FOD }(\mathrm{mM}) \\
\text { mean }(95 \% \mathrm{Cl})\end{array}$ & p-value \\
\hline \multicolumn{4}{|l|}{ SFA } \\
\hline C14.0 & $0.049(0.038-0.059)$ & $0.047(0.036-0.058)$ & 0.815 \\
\hline C16.0 & $12.376(11.846-12.906)$ & $11.501(10.418-12.584)$ & 0.092 \\
\hline C17.0 & $0.119(0.099-0.139)$ & $0.156(0.133-0.18)$ & 0.011 \\
\hline C18.0 & $8.474(8.027-8.92)$ & $6.043(5.342-6.745)$ & $<0.001$ \\
\hline C19.0 & $0.011(0.009-0.013)$ & $0.012(0.009-0.015)$ & 0.38 \\
\hline C20.0 & $0.038(0.036-0.04)$ & $0.033(0.029-0.038)$ & 0.05 \\
\hline C21.0 & $0.003(0.002-0.003)$ & $0.002(0.002-0.003)$ & 0.302 \\
\hline C22.0 & $0.085(0.079-0.09)$ & $0.067(0.062-0.072)$ & $<0.001$ \\
\hline C23.0 & $0.053(0.05-0.057)$ & $0.044(0.042-0.046)$ & $<0.001$ \\
\hline C24.0 & $0.061(0.058-0.065)$ & $0.066(0.062-0.071)$ & 0.054 \\
\hline $\mathrm{C} 25$ & $0.001(0.001-0.002)$ & $0.001(0.001-0.001)$ & $<0.001$ \\
\hline \multicolumn{4}{|l|}{ MUFA } \\
\hline C14.1n.5trans & $0.021(0.014-0.028)$ & $0.019(0.014-0.025)$ & 0.725 \\
\hline C14.1n.5 & $0.039(0.03-0.047)$ & $0.043(0.036-0.05)$ & 0.327 \\
\hline C16.1n.7trans & $0.027(0.024-0.03)$ & $0.037(0.03-0.045)$ & 0.007 \\
\hline C16.1n.7 & $0.11(0.096-0.123)$ & $0.077(0.057-0.097)$ & 0.006 \\
\hline C16.1n.9 & $0.236(0.201-0.27)$ & $0.303(0.236-0.37)$ & 0.044 \\
\hline C17.1n.7trans & $0.021(0.017-0.025)$ & $0.023(0.019-0.028)$ & 0.322 \\
\hline C17.1n.7 & $0.028(0.024-0.031)$ & $0.027(0.017-0.037)$ & 0.868 \\
\hline C18.1n.9trans & $0.045(0.039-0.051)$ & $0.036(0.033-0.04)$ & 0.01 \\
\hline C18.1n.7.trans & $0.029(0.025-0.033)$ & $0.027(0.021-0.033)$ & 0.566 \\
\hline C18.1n.12trans & $0.012(0.009-0.015)$ & $0.005(0.003-0.008)$ & 0.001 \\
\hline C18.1n.9 & $4.895(4.556-5.235)$ & $4.983(4.446-5.519)$ & 0.731 \\
\hline C22.1n.9 & $0.014(0.005-0.023)$ & $0.006(0.002-0.011)$ & 0.08 \\
\hline C24.1n.9 & $0.185(0.171-0.199)$ & $0.231(0.207-0.255)$ & 0.002 \\
\hline \multicolumn{4}{|l|}{ PUFA } \\
\hline C18.2n.6.tt & $0.016(0.014-0.018)$ & $0.01(0.009-0.012)$ & $<0.001$ \\
\hline C18.2n.6cc & $9.87(9.382-10.357)$ & $6.27(5.338-7.201)$ & $<0.001$ \\
\hline C18.3n.6 & $0.056(0.053-0.06)$ & $0.024(0.019-0.028)$ & $<0.001$ \\
\hline
\end{tabular}




\begin{tabular}{llll} 
C20.2n.6 & $0.088(0.072-0.104)$ & $0.065(0.06-0.071)$ & 0.006 \\
C20.3n.6 & $0.71(0.588-0.832)$ & $0.374(0.347-0.401)$ & $<0.001$ \\
C20.4n.6 & $5.624(5.273-5.974)$ & $1.193(0.896-1.49)$ & $<0.001$ \\
C22.2n.6 & $0.046(0.042-0.05)$ & $0.067(0.051-0.083)$ & 0.009 \\
C24.1n.9 & $0.185(0.171-0.199)$ & $0.231(0.207-0.255)$ & 0.002 \\
C22.4n.6 & $0.057(0.053-0.061)$ & $0.015(0.014-0.017)$ & $<0.001$ \\
C22.5n.6 & $0.256(0.231-0.281)$ & $0.046(0.04-0.051)$ & $<0.001$ \\
C24.4n.6 & $0.036(0.028-0.045)$ & $0.048(0.033-0.062)$ & 0.115 \\
C24.5n.6 & $0.009(0.008-0.01)$ & $0.005(0.004-0.006)$ & $<0.001$ \\
C18.3n.3 & $0.055(0.048-0.061)$ & $0.318(0.175-0.462)$ & 0.001 \\
C18.4n.3 & $0.001(0.001-0.001)$ & $0.003(0.002-0.004)$ & $<0.001$ \\
C20.3n.3 & $0.017(0.015-0.019)$ & $0.04(0.027-0.053)$ & 0.001 \\
C20.4n.3 & $0.004(0.003-0.005)$ & $0.141(0.082-0.201)$ & $<0.001$ \\
C20.5n.3 & $0.108(0.086-0.129)$ & $2.651(1.707-3.594)$ & $<0.001$ \\
C22.3n.3 & $0.029(0.027-0.031)$ & $0.028(0.024-0.031)$ & 0.533 \\
C22.5n.3 & $0.089(0.082-0.096)$ & $0.325(0.266-0.385)$ & $<0.001$ \\
C22.6n.3 & $2.682(2.559-2.805)$ & $3.739(3.356-4.122)$ & $<0.001$ \\
C24.5n.3 & $0.004(0.004-0.004)$ & $0.009(0.008-0.01)$ & $<0.001$ \\
C24.6n.3 & $0.031(0.024-0.037)$ & $0.051(0.04-0.061)$ & 0.002 \\
\hline
\end{tabular}

Table S6. Red blood cell characteristics in dams after being fed a control diet (CD) or fish oil diet (FOD) during gestation and lactation. Characteristics were measured in whole blood obtained from dams at the end of lactation (on postnatal day 21). RBC: red blood cell count; $\mathrm{Hb}$ : hemoglobin; $\mathrm{MCH}$ : mean corpuscular hemoglobin; RDW: relative distribution width; MCV: mean corpuscular volume.

\begin{tabular}{llllll}
\hline & $\mathbf{R B C}(* \mathbf{1 0} \mathbf{1 2} \mathbf{L})$ & $\mathbf{H b}(\mathbf{m M})$ & MCH $(\mathbf{f m o l})$ & $\mathbf{R D W}(\%)$ & MCV (fL) \\
\hline Dam \#1 (CD) & 10.22 & 9.8 & 0.96 & 23.9 & 51 \\
Dam \#2 (FOD) & 7.25 & 6.6 & 0.91 & 28.3 & 45 \\
Dam \#3 (FOD) & 8.37 & 7.4 & 0.88 & 30.6 & 45 \\
\hline
\end{tabular}




\section{Th1 polarization assay}
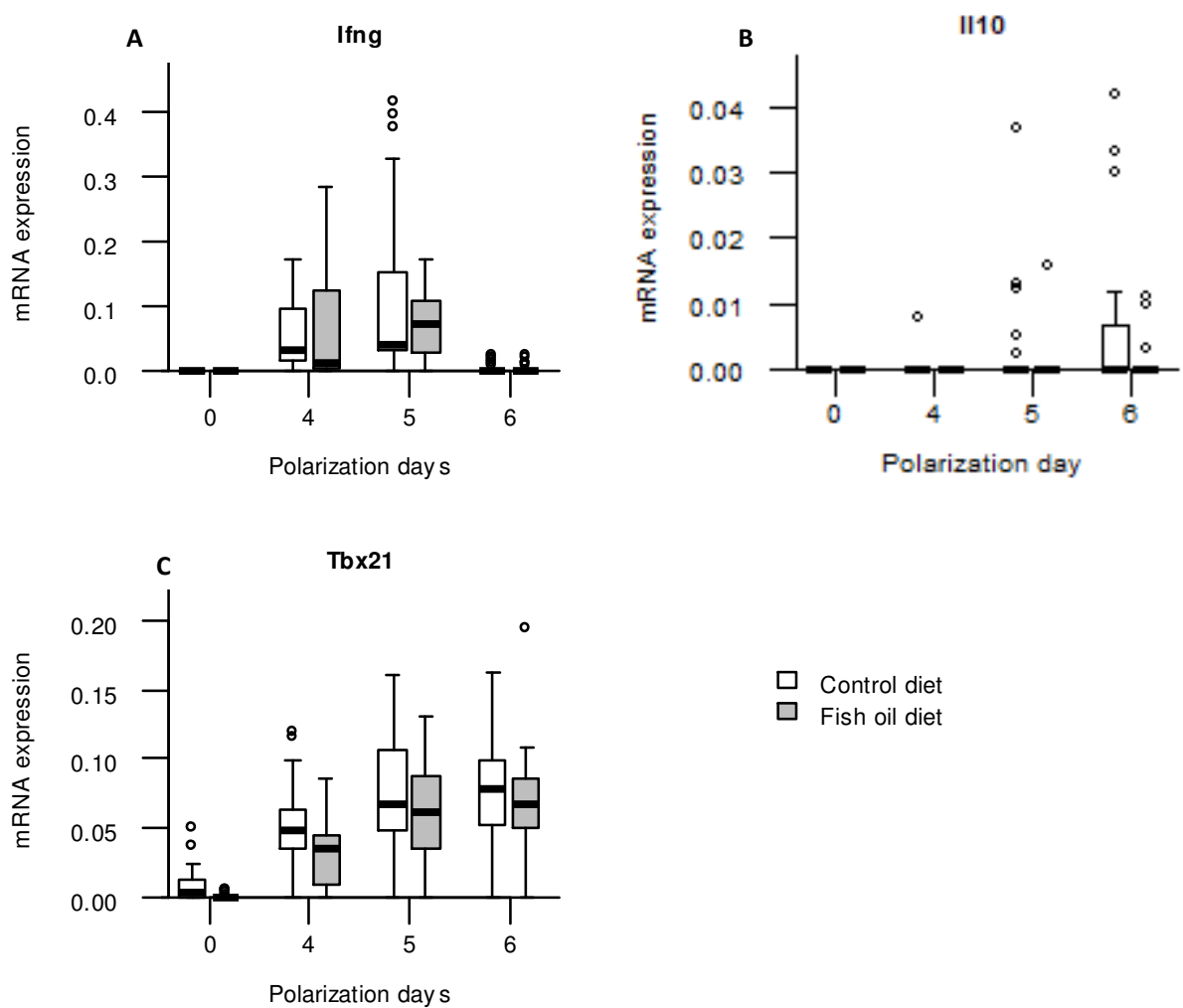

$\square$ Control diet

$\square$ Fish oil diet

Figure S2. Effect of perinatal fish oil diet (FOD) on ex vivo Th1 polarization of naïve $\mathrm{CD}^{+}{ }^{+} \mathrm{T}$ cells. mRNA expression of Ifng (a), II10 (B) and Tbx21 (C) were measured at 0, 4, 5 and 6 days after incubation of naïve $C D 4^{+} T$ cells in presence of $\alpha-C D 3, \alpha-C D 28, I L-2$ and IL-12. Splenic naïve CD4 ${ }^{+} T$ cells were isolated at the end of lactation from offspring after dams received a control diet (CD) or FOD during gestation and lactation. Data are presented as boxplots. Statistical analysis by linear mixed-effects model. $n=$ $32-35$ vs. $25-27$ (CD vs. FOD). 


\section{Th2 polarization assay}
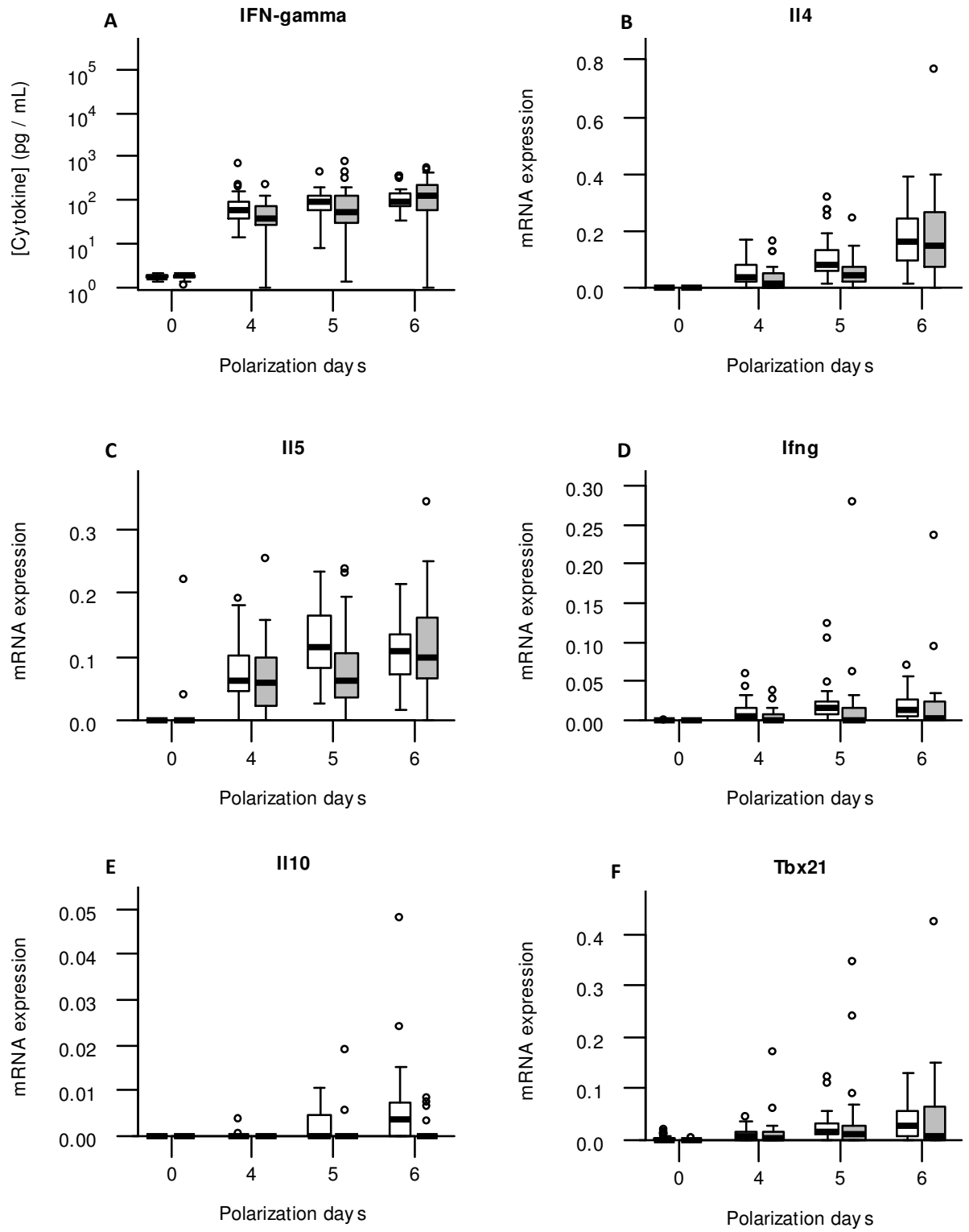

Figure S3 (continued on next page). 


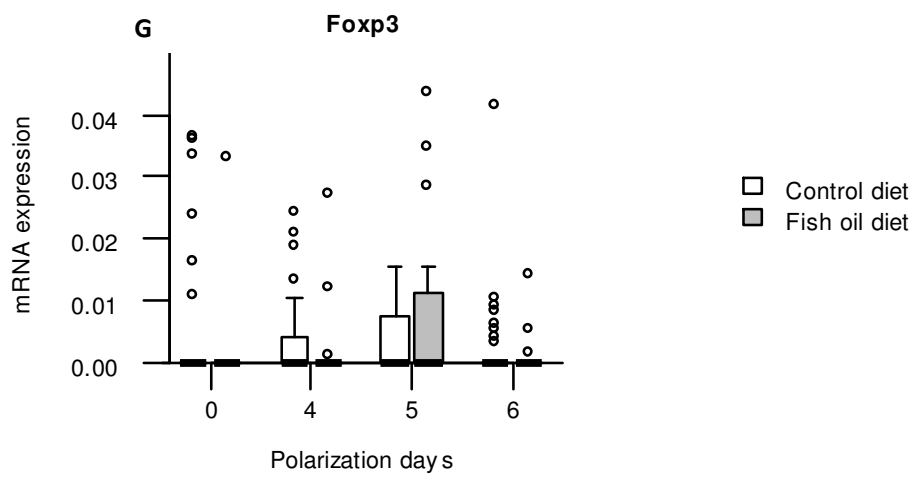

Figure S3. Effect of perinatal fish oil diet (FOD) on ex vivo Th2 polarization of naive $\mathrm{CD}^{+}{ }^{+}$cells. Extracellular IFN- $\gamma(\mathrm{A})$, and mRNA expression of $/ 14$ (B), $/ 15$ (C), Ifng (D), $/ 110$ (E), Tbx21 (F) and Foxp3 (G) were measured at $0,4,5$ and 6 days after incubation of naïve $C D 4^{+} T$ cells in presence of $\alpha$-CD3, $\alpha$ CD28, IL-2 and IL-4. Splenic naïve CD4 ${ }^{+} \mathrm{T}$ cells were isolated at the end of lactation from offspring after dams received a control diet (CD) or FOD during gestation and lactation. Data are presented as boxplots. Statistical analysis by linear mixed-effects model. $n=n=27-32$ vs. 20-25 (CD vs. FOD). 


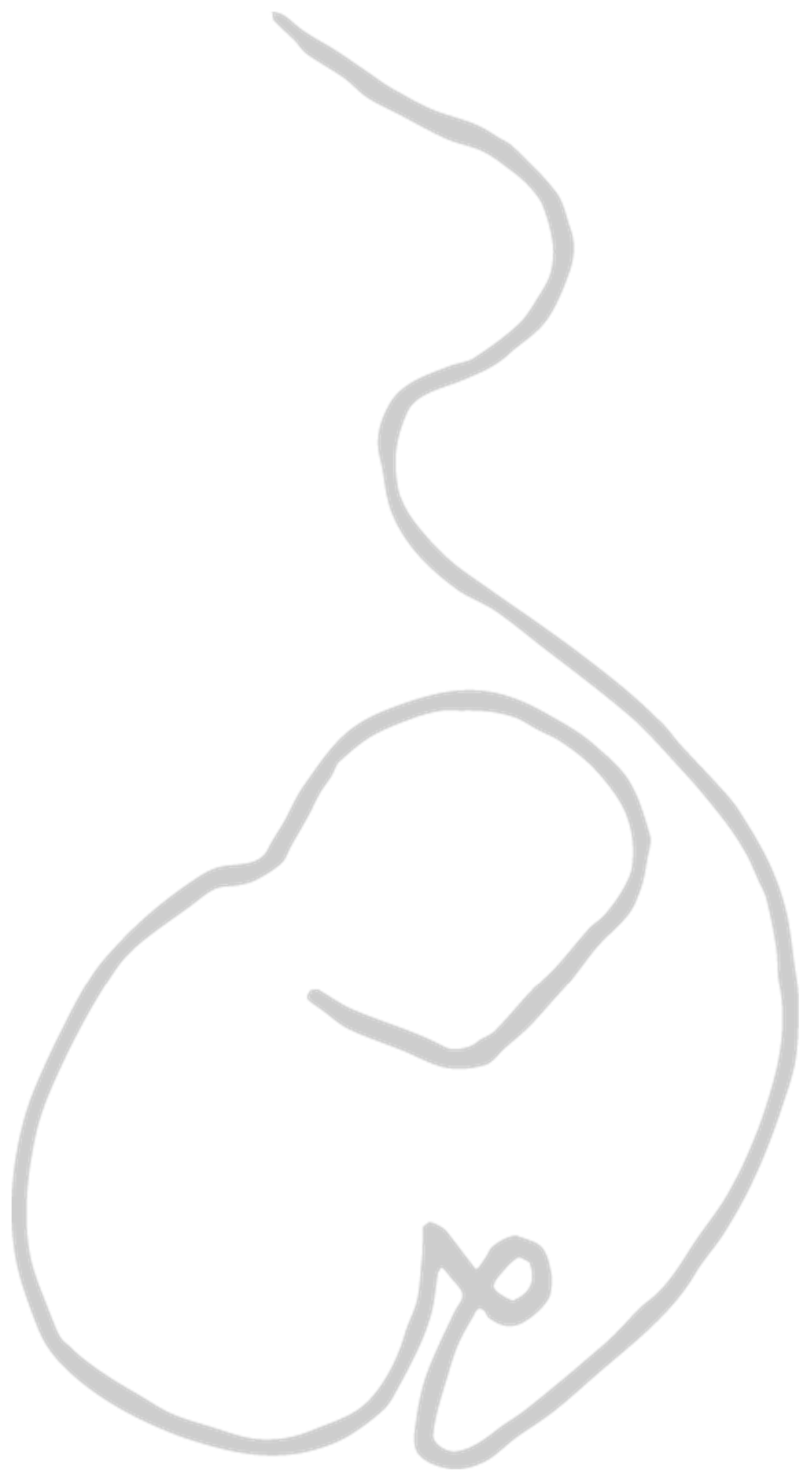

112 


\section{Chapter 5}

\section{Maternal fatty acid status during pregnancy versus offspring inflammatory markers: a canonical correlation analysis of the MEFAB cohort}

Sven H. Rouschop, Agnieszka Smolinska, Marij Gielen, Renate de Groot, Maurice Zeegers, Antoon Opperhuizen, Frederik J. van Schooten, Roger W. Godschalk

In preparation for submission 


\begin{abstract}
The development of inflammatory lung disorders in children is related to maternal diet during pregnancy. We therefore aimed to examine maternal fatty acid (FA) status and its associations with inflammatory markers and lung conditions in the child. This was done by analyzing data from the MEFAB cohort using multivariate canonical correlation analysis (CCA). In the MEFAB cohort, maternal plasma phospholipid FAs were measured at 16, 22 and 32 weeks of pregnancy, and at day of birth. Child inflammatory markers and selfreported doctor diagnosis of inflammatory lung conditions were assessed at 7 years of age. Using CCA, we found that maternal FA levels during pregnancy were significantly associated with child inflammatory markers at 7 years of age and that Mead acid was the most important FA for this correlation. To further verify the importance of Mead acid, we examined the relation between maternal Mead acid levels at day of birth with the development of inflammatory lung disorders in children. After stratification for the child's sex, maternal Mead acid levels at day of birth were significantly related with self-reported doctor diagnosis of asthma and lung infections in boys, and bronchitis and total number of lung conditions in girls. Future studies should investigate whether the importance of Mead acid in the relation between maternal FA status and inflammation and lung conditions in the child is due to its role as biomarker for essential fatty acid deficiency or due to its own biological function as pro-inflammatory mediator.
\end{abstract}




\section{Introduction}

Inflammatory lung diseases are a major burden to global health. According to the World Health Organization (WHO), respiratory infections are the fourth leading cause of both death and burden of disease in the world [1]. Similarly, asthma prevalence has increased over the past few decades [2-4], making asthma the most common chronic disease in children [5]. The development of inflammatory lung disorders in children has been related to changes in maternal diet during pregnancy. Animal studies have demonstrated that changes in maternal diet during gestation predispose weanling offspring to airway inflammation, airway hyperresponsiveness, and increased susceptibility to Respiratory Syncytial Virus (RSV) infection [6,7]. Likewise, both observational and intervention studies in humans have shown that the risk of having asthma and asthma-related symptoms in offspring is negatively correlated with maternal intake of $n-3$ polyunsaturated fatty acids (PUFAs) [8-13]. Furthermore, maternal fatty acid (FA) status during pregnancy has been linked to inflammation in general and the development of the immune system in the child [14].

When examining maternal FA status during pregnancy and its relation with offspring health, FA levels are often estimated through the use of food frequency questionnaires (FFQs) $[9,15]$, rather than using objective FA biomarkers, such as plasma phospholipids. Furthermore, studies usually assess maternal FAs at a single prenatal time point $[16,17]$, instead of measuring them throughout pregnancy. Moreover, the relation between maternal FA status and offspring health is commonly studied by analyzing single associations between an individual exposure variable and an individual outcome parameter [9,15-17]. However, the complex etiology of biological disorders may be better studied by examining a set of exposure variables and a set of outcome parameters simultaneously [18].

The aim of this study was to examine maternal FA status and its associations with inflammation and lung conditions in the child, while averting the aforementioned shortcomings. This was done by analyzing data from the Maastricht Essential Fatty Acid Birth (MEFAB) cohort using canonical correlation analysis (CCA). The MEFAB cohort was established to study the associations between maternal FA status during pregnancy and pregnancy outcomes [19]. In this cohort, maternal plasma phospholipid FAs were measured at 16, 22 and 32 weeks of pregnancy, and at day of birth, and child inflammatory markers were measured at 7 years of age. CCA is a statistical model which assesses the multivariate correlation between two sets of variables. Although used in many research areas, such as social and behavioral research [20], bioinformatics [21], and genetics [22], CCA has not been used to study the relation between maternal fatty acid status and child inflammation before. 
This paper shows that maternal plasma FA levels during pregnancy are significantly associated with child inflammatory markers at 7 years of age and that the FA Mead acid is most important for this correlation. In addition, we show that maternal Mead acid levels at day of birth are significantly correlated with self-reported doctor diagnosis of various inflammatory lung conditions in the child.

\section{Methods}

A schematic overview of the study design and analysis workflow are presented in Figure 1.

\section{Study design, setting and participants}

Pregnant women attending one of three antenatal clinics in the south the Netherlands between 1989 and 1995 were asked to participate in the cohort. Women were eligible for participation if they were less than 16 weeks pregnant and did not suffer from any cardiovascular, neurological, renal or metabolic condition. Originally, 1203 women were included in the MEFAB cohort. For the follow-up, 305 singletons born between 1990 and 1994 were included between 1997 and 2000. For the current analysis, only mother-child pairs were included for which there were no missing data in any of the maternal plasma fatty acid concentrations or child plasma inflammatory markers, leading to an $n$ of 173

Pregnancy Child

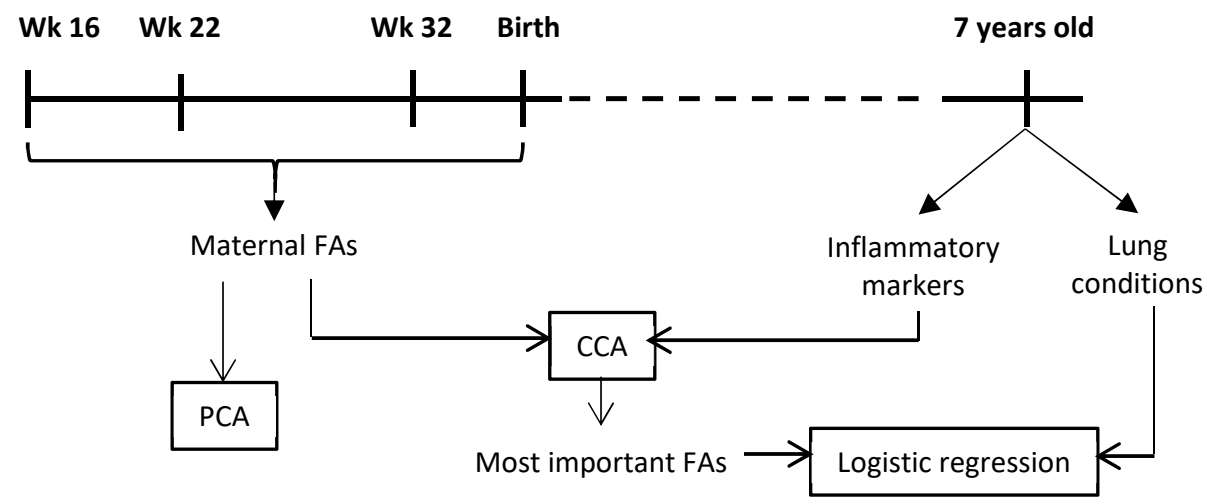

Figure 1. Schematic overview of the study design and analysis workflow. Wk, weeks of pregnancy; FAs, fatty acids; PCA, principle component analysis; CCA, canonical correlation analysis. 
mother-child pairs. The study was approved by the medical ethics committee of the University hospital Maastricht and Maastricht University. Written consent was given by pregnant women before enrolment in the initial study and again by both parents (if possible) for the follow-up study.

\section{Exposure variables}

To measure maternal FA status during pregnancy, maternal venous blood samples were collected in EDTA tubes around 16, 22 and 32 weeks of pregnancy, and immediately after delivery. Concentrations of 39 different plasma phospholipid FAs were determined by capillary gas-liquid chromatography as described previously [23] and expressed in $\mathrm{mg} / \mathrm{L}$. See Table S1 for a list of the FAs that were measured.

\section{Outcome variables}

At seven years of age, offspring inflammatory markers were measured in plasma. These markers included: total white blood cell, monocyte, granulocyte and lymphocyte count ( $\left.\times 10^{9} / \mathrm{L}\right)$ determined by Beckman-Coulter Gen-s (Beckman Coulter, Brea, CA, USA); tissue plasminogen activator (tPA) concentration $(\mathrm{ng} / \mathrm{ml}$ ) and enzyme activity $(\mathrm{IU} / \mathrm{ml})$ measured by ELISA and bioimmunoassay, respectively (Biopool International, Ventura, CA, USA); plasminogen activator inhibitor-1 (PAI-1) concentration $(\mathrm{ng} / \mathrm{ml})$; leptin concentration $(\mu \mathrm{g} / \mathrm{l})$ determined by RIA (Linco Research, St Charles, MO, USA); fibrinogen concentration ( $\mathrm{g} / \mathrm{l}$ ) measured by the Clauss method [24]; and C-reactive protein (CRP) concentration (mg/l) assayed with an in-house ELISA using polyclonal antibodies as catching and tagging antibodies labelled with HRP (Dako, Glostrup, Denmark).

To assess the children's history of lung conditions, their parents were asked at follow-up whether their child was ever diagnosed by a medical doctor with asthma, bronchitis, or pneumonia which was treated with antibiotics.

\section{Confounding variables}

Information on potential confounders was obtained using hospital records for maternal age (years) and BMI $\left(\mathrm{kg} / \mathrm{m}^{2}\right)$ at study entry, duration of pregnancy (days), child sex, and child birthweight (grams). Information regarding maternal smoking and alcohol consumption during pregnancy (yes or no), breastfeeding (yes or no) with or without a combination of formula feeding, presence of pets (dog, cat, rodent or bird) at home during childhood (yes or no), and daycare attendance during first four years (yes or no) was obtained from 
questionnaires. Child BMI at seven years of age $\left(\mathrm{kg} / \mathrm{m}^{2}\right)$ was assessed by a nurse during a follow-up visit.

\section{Canonical correlation analysis}

The relation between maternal FA status and child inflammatory status was studied using CCA. CCA is a method which explores the linear relationship between two multivariate data sets $X$ and $Y$ (equation 1 ). CCA does this by making separate linear combinations (called canonical variates) of both data sets by multiplying each separate variable in $\mathrm{X}$ or $\mathrm{Y}$ with a coefficient (a so-called canonical weight) and taking the sum of these products (equation 2). Canonical weights are chosen in such a way that the correlation between the canonical variates of $X$ and $Y$ is maximized. CCA develops as many pairs of canonical variates as there are variables in the smallest of the two data sets. Furthermore, all canonical variates are orthogonal (independent) of each other, to make sure that each pair of canonical variates represents a different relationship between $\mathrm{X}$ and $\mathrm{Y}$. To test if the correlation between two canonical variates is significant, an asymptotic test using F-approximation of Wilks' Lambda was applied. Only canonical variate pairs with a significant correlation were used for biological interpretation. Individual variables were considered to have a considerable contribution to a canonical correlation if their canonical loadings, which represent the correlation between the individual variable and the conical variate, were $>|0.3|$.

$$
X=\left(\begin{array}{c}
x_{1} \\
x_{2} \\
\cdots \\
x_{p}
\end{array}\right) \quad \mathrm{Y}=\left(\begin{array}{c}
y_{1} \\
y_{2} \\
\cdots \\
y_{q}
\end{array}\right)
$$

$$
\begin{array}{cc}
U_{1}=a_{11} x_{1}+a_{12} x_{2}+\cdots+a_{1 p} x_{p} & V_{1}=b_{11} y_{1}+b_{12} y_{2}+\cdots+b_{1 p} y_{p} \\
U_{2}=a_{21} x_{1}+a_{22} x_{2}+\cdots+a_{2 p} x_{p} & V_{2}=b_{21} y_{1}+b_{22} y_{2}+\cdots+b_{2 p} y_{p} \\
\cdots & \cdots \\
U_{p}=a_{p 1} x_{1}+a_{p 2} x_{2}+\cdots+a_{p p} x_{p} & V_{p}=b_{p 1} y_{1}+b_{p 2} y_{2}+\cdots+b_{p p} y_{p}
\end{array}
$$

Equation 1 and 2. Canonical correlation analysis explores the linear relationship between two multivariate data sets $X$ and $Y$ (equation 1 ). It does so by making separate linear combinations (canonical variates) of both data sets by multiplying each separate variable in $\mathrm{X}$ or $\mathrm{Y}$ with a canonical weight and taking the sum of these products (equation 2). 


\section{Model optimization}

First a full CCA model was obtained using all 39 maternal fatty acids as independent variables and all child inflammatory markers as dependent variables (Figure 2A). To further identify which specific fatty acids were most important for this relation, the number of independent variables in the model was reduced by deleting the fatty acid variable with the

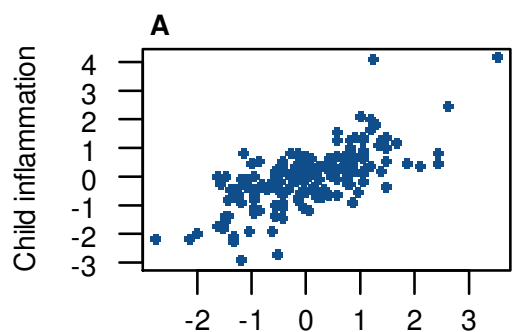

B

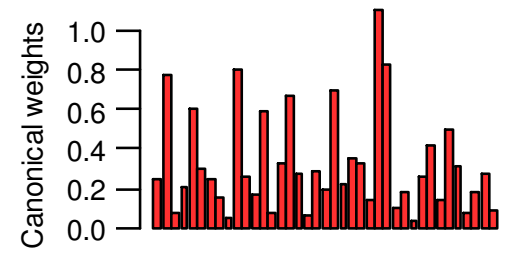

Maternal fatty acids
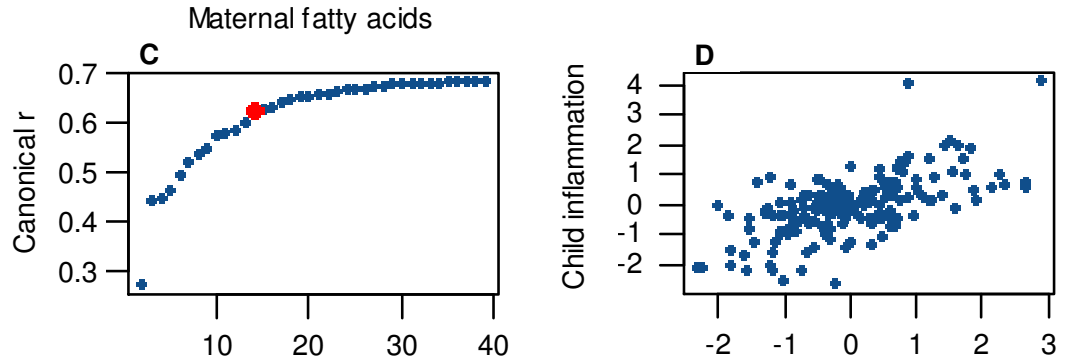

No. of FA in model

E

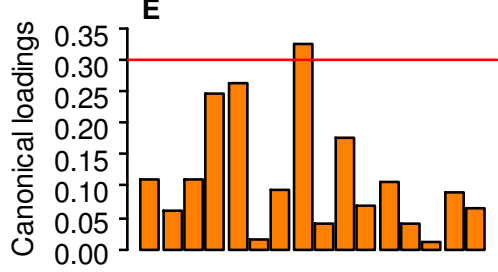

Maternal fatty acids

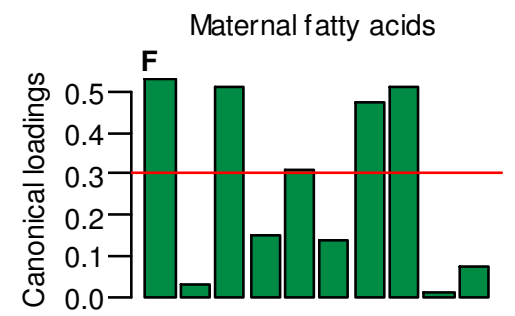

Child inflammatory markers

Figure 2. Analysis strategy for performing canonical correlation analysis (CCA), reducing the number of independent variables, obtaining an optimal model, and selecting the most important variables. A) A full CCA model was obtained, containing all 39 maternal fatty acids vs. all child inflammatory markers. B) To reduce the number of independent variables in the CCA model, the fatty acid variable with the lowest absolute, standardized weight was eliminated and the analysis was repeated. C) The process of removing the least contributing independent variable and rerunning the analysis was iterated until an optimum was achieved between a low number of variables and a high correlation. This optimum was set at the point where the canonical correlation coefficient $(r)$ starts decreasing with increasing intervals while cutting out independent variables (indicated by the red dot). D) With the optimal number of independent variables, an optimal CCA model was made. E-F) Fatty acids and inflammatory markers with an absolute loading $>0.3$ (cut-off indicated by the red line) were selected as most important for the canonical correlation. 
smallest contribution to the model (i.e. with the smallest absolute, standardized canonical weight) and the analysis was repeated (Figure 2B). By removing variables, information is withdrawn from the model and the correlation decreases. Therefore, this process of removing the least contributing independent variable was iterated until an optimal balance was achieved between a low number of independent variables and a high correlation (Figure 2C). This optimum was chosen to be at the point where the rate of decrease in correlation due to a diminishing number of independent variables suddenly increased, i.e. where the correlation coefficient suddenly started dropping quicker. With this optimal number of independent variables, an optimal CCA model was made (Figure 2D). For all optimized CCA models, the most important maternal fatty acids and child inflammatory markers were identified by selecting variables with an absolute canonical loading $>|0.3|$ (Figure 2E-F).

\section{Logistic regression}

The relation between maternal FA variables with a canonical loading $>|0.3|$ and offspring lung disorders was assessed using binomial and ordinal logistic regression. The influence of potential confounders on logistic regression was determined by including the potential confounder as a covariate in the analysis. If inclusion of the confounder led to a significant covariate effect, the main effect not being significant anymore, or a change $>10 \%$ of the main effect odds ratio, the variable was considered to be a confounder. For all statistical analyses, $p<0.05$ was considered significant. All statistical analyses were performed using $R$ version 4.0.2 (R Foundation for Statistical Computing, Vienna, Austria). CCA was done with CCA package version 1.2.

\section{Results}

\section{Population characteristics}

Practically all mothers included in the analysis were Caucasian (99\%; Table 1). On average, mothers were 30 years-old when entering the study and had a BMI of $24 \mathrm{~kg} / \mathrm{m}^{2}$ and a normal pregnancy duration (279 days). Twenty percent of the mothers smoked during pregnancy, with a mean of nine cigarettes per day. Three percent consumed alcohol during pregnancy, but none specified how much. Deliveries were mostly vaginal (95\%) and half of the infants were breastfed for some period of time (47\%). About half of the children were female (43\%). On average, children had a normal birthweight (3362 grams) and BMI at seven years-of-age $\left(15 \mathrm{~kg} / \mathrm{m}^{2}\right)$. 
Table 1. Population characteristics of the MEFAB participants included in this study ( $n=173$ motherchild pairs). SD, standard deviation; IQR, interquartile range.

\begin{tabular}{lll}
\hline Variable & Mean (SD) & Median (IQR) \\
\hline Mother & 99.41 & \\
Ethnicity (Caucasian \%) & $30.12(4.14)$ & $29.54(5.33)$ \\
Age at entry study (years) & $23.56(3.75)$ & $23.05(3.66)$ \\
BMI at entry study (kg/m²) & & \\
Pregnancy & & $281(12)$ \\
Duration (days) & $279(11)$ & \\
Smoking (\%) & 19.77 & \\
$\quad$ Cigarettes per day & $9.17(5.62)$ & \\
Alcohol consumption (\%) & 2.92 & \\
Caesarian section (\%) & 4.62 & \\
Breastfeeding (\%) & 46.82 & \\
& & 3400 (640) \\
Child & & $15.03(2.12)$ \\
Sex (Female \%) & 43.35 & \\
Birthweight (grams) & $3362(495)$ & \\
BMI at 7 years & $15.47(1.88)$ & \\
Asthma (\%) & 6.98 & \\
Bronchitis (\%) & 22.22 & \\
Lung infection (\%) & 7.06 & \\
\hline
\end{tabular}

\section{Maternal plasma fatty acid status changes throughout pregnancy}

To assess the progression of maternal fatty acid status during pregnancy, PCA was applied to maternal plasma concentrations of 39 different FAs during pregnancy. The result from this is shown in Figure 3, with each dot representing a mother, with different colors representing different prenatal time points and the position of the dot representing the overall FA status. The gradual shift of the cloud of dots from week 16 to week 22, to week 32 , to partus indicates that the overall maternal FA profile gradually changed throughout pregnancy, with the largest shift occurring between 16 and 22 weeks of pregnancy.

\section{Maternal fatty acid status during pregnancy correlates with child inflammatory status at seven years of age}

To assess the multivariate relation between maternal FA levels during pregnancy and inflammatory markers in the child at seven years of age, CCA was used. Since PCA showed that the maternal fatty acid status gradually changed throughout pregnancy, a separate CCA 


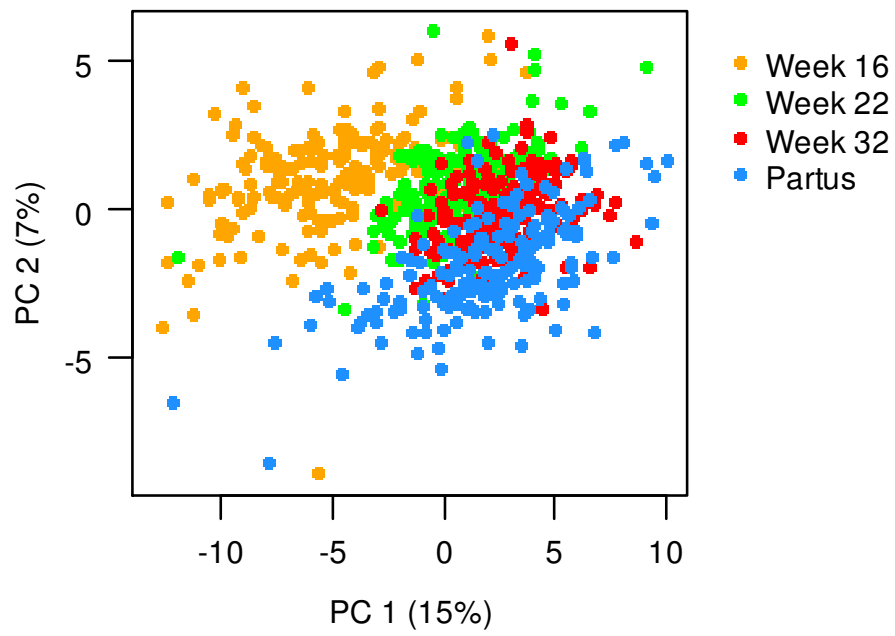

Figure 3. Principle component analysis of 39 maternal plasma fatty acid concentrations, measured at 16, 22 and 32 weeks of pregnancy, and at day of birth (partus). Data are presented as scatter plot of the first and second principle component (PC), covering $15 \%$ and $5 \%$, respectively, of the total variance, with each dot representing one mother at a specific gestational time point $(n=173$ mothers).

was performed for each prenatal time point. The optimized CCA models for week 16, week 22 and week 32 of pregnancy and partus contained sixteen, fourteen, fifteen and nineteen independent FA variables, respectively (Table 2). For all these optimized CCA models, the first canonical variates represented significant, moderate canonical correlations (week 16: $r=0.60, p<0.001$; week 22: $r=0.62, p<0.001$; week 32: $r=0.64 ; p=0.001$; partus: $r=0.61$, $p=0.028$; Table 2). All lower-order canonical variates were not significant and thus not used for further biological interpretation (Table S2). For each significant canonical variate, the most important independent and dependent variables were identified by selecting variables with an absolute canonical loading $>0.3$. Of these various fatty acids and inflammatory markers that were identified to be important for the different canonical correlations, Mead acid (20:3n9) and granulocyte count, respectively, occurred most often (Table 2).

\section{Maternal Mead acid levels at day of birth are positively associated with the development of lung conditions in offspring}

Since Mead acid occurred most often as important fatty acid variable in the CCA, the relevance of maternal Mead acid levels during pregnancy was further assessed by examining the association between maternal plasma levels of Mead acid at day of birth and the odds of the child developing asthma, bronchitis or lung infection during the first seven years of age. Mead acid data from day of birth were used, since Mead acid concentrations 
Table 2. Canonical correlation analysis of maternal plasma fatty acid (FA) concentrations at 16, 22 and 32 weeks of pregnancy, and at day of birth (partus) versus child plasma inflammatory markers at seven years of age ( $n=173$ mother-child pairs). $r$, canonical correlation coefficient.

\begin{tabular}{|c|c|c|c|c|c|}
\hline & $\begin{array}{l}\text { No. of FAs } \\
\text { in CCA model }\end{array}$ & $r$ & p-value & $\begin{array}{l}\text { FAs } \\
\mid \text { loading |>0.3 }\end{array}$ & $\begin{array}{l}\text { Inflammatory markers } \\
\mid \text { loading } \mid>0.3\end{array}$ \\
\hline Week 16 & 16 & 0.60 & $<0.001$ & $\begin{array}{l}\text { 24:1n9 } \\
\text { 18:1DMA }\end{array}$ & $\begin{array}{l}\text { Monocytes } \\
\text { Granulocytes } \\
\text { tPA activity }\end{array}$ \\
\hline Week 22 & 14 & 0.62 & $<0.001$ & $20: 3 n 9$ & $\begin{array}{l}\text { White blood cells } \\
\text { Granulocytes } \\
\text { PAl-1 } \\
\text { Leptin }\end{array}$ \\
\hline Week 32 & 15 & 0.64 & 0.001 & $\begin{array}{l}22: 2 \mathrm{n} 6 \\
16: 0 \mathrm{DMA} \\
20: 3 n 9\end{array}$ & $\begin{array}{l}\text { White blood cells } \\
\text { Granulocytes } \\
\text { tPA activity } \\
\text { tPA concentration } \\
\text { PAI-1 }\end{array}$ \\
\hline Partus & 19 & 0.61 & 0.028 & $\begin{array}{l}20: 3 n 9 \\
24: 1 n 9 \\
22: 2 n 6 \\
16: 0 D M A\end{array}$ & $\begin{array}{l}\text { White blood cells } \\
\text { Granulocytes } \\
\text { tPA concentration } \\
\text { PAI-1 } \\
\text { CRP }\end{array}$ \\
\hline
\end{tabular}

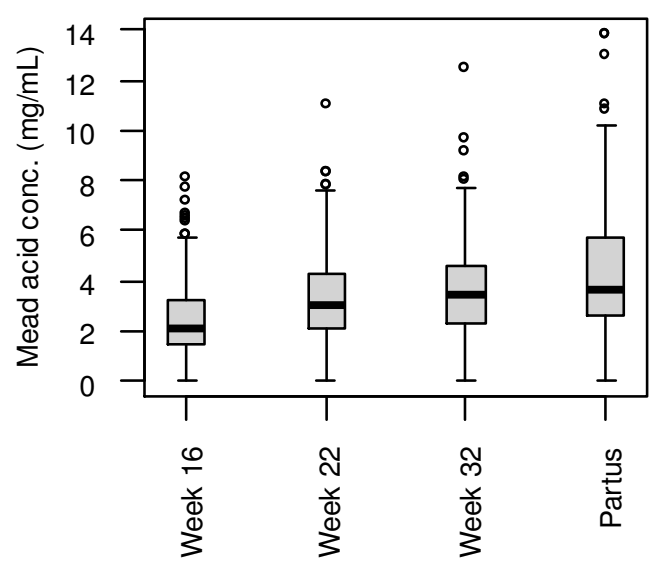

Figure 4. Mead acid concentrations in maternal plasma at 16, 22 and 32 weeks of pregnancy, and at day of birth (partus). Data are presented as boxplots, with dots representing values higher than 1.5 times the interquartile range above the third quartile ( $n=173$ mothers).

increased throughout pregnancy, with the highest concentrations and the largest variation occurring at that specific time point (Figure 4). Using unadjusted binomial regression, a significant, positive relation was found between maternal Mead acid levels and self- 
reported doctor diagnosis of bronchitis $(\mathrm{OR}[95 \% \mathrm{Cl}]=1.17[1.01-1.34])$ and lung infection (OR $[95 \% \mathrm{Cl}]=1.27[1.04-1.55]$ ), but not asthma (Table 3). Furthermore, unadjusted ordinal logistic regression showed a significant, positive association between maternal Mead acid concentrations at day of birth and the total number of self-reported doctordiagnosed lung conditions that the child suffered from during the first seven years of age $(\mathrm{OR}[95 \% \mathrm{Cl}]=1.18$ [1.03 - 1.34]; Table 3). Adjusting the logistic regression models for maternal age or BMI at study entry, gestational age, smoking or alcohol use during pregnancy, birthweight, breastfeeding, presence of pets, daycare attendance, or child BMI at seven years of age left the effect estimate and significance of Mead acid largely unchanged (Table S3). Stratification for child sex, however, did lead to sex-specific results. For asthma, bronchitis and number of lung conditions, associations were significant for girls (OR [95\% Cl] =1.77 [1.09-3.62], 1.32 [1.03 - 1.72], and 1.40 [1.10 - 1.80], respectively) but not boys, whereas for lung infection, associations were significant for boys $(\mathrm{OR}[95 \% \mathrm{Cl}]=$ 1.28 [1.02 - 1.63]), but not girls (Table 3).

Table 3. Associations between maternal plasma mead acid concentration at day of birth and selfreported doctor diagnosis of lung conditions in the child during the first seven years of age $(n=173$ mother-child pairs). Statistical analysis by binomial logistic regression (for asthma, bronchitis and lung infection) or ordinal logistic regression (for number of lung conditions). OR (odds ratio), $95 \% \mathrm{Cl}$ (confidence interval) and $\mathrm{p}$-value are shown for both unadjusted results and results stratified for child sex.

\begin{tabular}{llll}
\hline & OR & 95\% Cl & p-value \\
\hline Asthma & & & \\
Unstratified & 1.13 & $0.91-1.38$ & 0.228 \\
Girls & 1.77 & $1.09-3.62$ & 0.038 \\
Boys & 1.03 & $0.77-1.29$ & 0.842 \\
Bronchitis & & & \\
Unstratified & 1.17 & $1.01-1.34$ & 0.030 \\
Girls & 1.32 & $1.03-1.72$ & 0.029 \\
Boys & 1.11 & $0.93-1.33$ & 0.227 \\
Lung infection & & & \\
Unstratified & 1.27 & $1.04-1.55$ & 0.014 \\
Girls & 1.27 & $0.82-1.91$ & 0.237 \\
Boys & 1.28 & $1.02-1.63$ & 0.029 \\
Number of lung conditions & & & \\
Unstratified & 1.18 & $1.03-1.34$ & 0.007 \\
Girls & 1.40 & $1.10-1.80$ & 0.003 \\
Boys & 1.12 & $0.94-1.31$ & 0.093 \\
\hline
\end{tabular}




\section{Discussion}

The development of inflammatory lung disorders has previously been shown to be related to maternal diet during pregnancy. However, estimates of prenatal exposures and statistical analysis were often suboptimal in previous studies. Therefore, this study examined the relation between maternal FA status and child inflammation by analyzing data from the MEFAB cohort using CCA. This paper showed that maternal plasma FA levels during pregnancy were significantly associated with child inflammatory markers at 7 years of age and that Mead acid was the most important FA for this correlation. In addition, we showed that maternal Mead acid levels at day of birth were significantly correlated with selfreported asthma, bronchitis and the total number of lung conditions in girls, and with selfreported lung infection in boys.

First, we showed that the overall profile of maternal FAs gradually changed throughout pregnancy. This finding emphasizes the importance of assessing FA levels at consistent prenatal time points when studying the relation between maternal FA status and offspring health, rather than assessing FA exposure at varying prenatal time points, as has been the case for previous studies. For instance, Notenboom et al. measured maternal FA levels in the 34th to 36th week of gestation [16], whereas Rucci et al. measured maternal FA levels around the $20^{\text {th }}$ week of gestation [17], making comparison of both studies suboptimal. Moreover, Miyake et al. assessed maternal FA consumption at study entry, irrespective of the gestational age [9], thereby potentially introducing heterogeneity in the exposure data set. The current study, in contrast, measured maternal FA levels at 16, 22 and 32 weeks of gestation, and at day of birth, thereby covering both the second and third trimester. In addition, maternal FA status was assessed by measuring plasma phospholipid FAs, which represent FA levels of the past few days [25]. Altogether, the exposure variables as assessed in the current study may be considered a comprehensive representation of prenatal maternal FA status.

Using CCA, we examined the multivariate relation between child inflammatory markers at 7 years of age and maternal plasma FA levels at 16, 22 and 32 weeks of gestation, and at day of birth. With this approach, we found that for all prenatal time points, maternal FA status was moderately, but significantly associated with child inflammatory markers at 7 years of age. In addition, Mead acid was identified most often as an important exposure variable for these multivariate correlations. Mead acid (20:3n-9) is an n-9 PUFA which is synthesized in vivo from oleic acid (18:1n-9) through elongation and desaturation by the enzymes Fatty acid desaturase 1 (Fads1), Fatty acid desaturase 2 (Fads2) and Elongation of very long chain fatty acids protein 5 (Elovl5) [26]. Fads1, Fads2 and Elovl5 also elongate and desaturate the n-6 and n-3 PUFAs linoleic acid (LA, 18:2n-6) and $\alpha$-linolenic acid (ALA, 18:3n- 
3) into their respective longer-chain derivatives, such as arachidonic acid (AA, 20:4n-6), eicosapentaenoic acid (EPA, 20:5n-3) and docosahexaenoic acid (DHA, 22:6n-3) [27]. As a result of these shared metabolic pathways between n-3, n-6 and n-9 PUFAs, Mead acid synthesis is enhanced when n-3 and n-6 PUFA levels are low, making Mead acid a biomarker for essential fatty acid deficiency [28]. Next to this, Mead acid has its own biological functions. Similar to the conversion of AA into 5-hydroxyeicosatetraenoic acid (5-HETE) by 5-lipoxygenase (5-LOX) and its subsequent oxidation into 5-oxo-eicosatetraenoic acid (5oxo-ETE) by 5 -hydroxyeicosanoid dehydrogenase (5-HEDH), Mead acid is metabolized into 5-hydroxyeicosatrienoic acid (5-HETrE) and 5-oxo-eicosatrienoic acid (5-oxo-ETrE), respectively, by the same enzymes $[29,30]$. In accordance with these structural analogies, 5-oxo-ETrE is as potent as 5-oxo-ETE in inducing chemotaxis, surface expression of CD11b and calcium mobilization in neutrophils, as well as increasing actin polymerization in eosinophils [29]. Mead acid thus acts as a pro-inflammatory mediator, thereby potentially actively contributing to the course of inflammatory lung conditions, such as lung infections, asthma and bronchitis.

Next to Mead acid being identified as an important exposure variable, the most frequently identified important outcome variable for the multivariate correlation between maternal FA status and child inflammation was granulocyte count. Granulocytes play an important role in various inflammatory lung conditions. For instance, both eosinophils and neutrophils are associated with clinical severity and pulmonary dysfunction in asthma patients [31-34]. Similarly, fungal infection of the lungs is associated with pulmonary influx of eosinophils [35], whereas neutrophils contribute to early defense against pulmonary infection with bacteria [36]. Considering the identification of both Mead acid and granulocyte count as important variables, and given the biological effects Mead acid can have on eosinophils and neutrophils, and the role these granulocytes play in inflammatory lung conditions, Mead acid may possibly exert any potential influence on lung conditions through its effect on granulocytes. Whether this hypothesis is correct and what molecular or cellular mechanisms would be involved in this, remains to be examined by future studies.

To further evaluate the relevance of maternal Mead acid levels during pregnancy, the association between maternal plasma levels of Mead acid at day of birth and the selfreported doctor diagnosis of lung conditions in children was examined. As a result, maternal Mead acid levels at day of birth were positively associated with the diagnosis of several lung conditions in children. After stratification for sex, these associations were significant for asthma, bronchitis and number of lung infections for girls but not boys, whereas for lung infection, associations were significant for boys but not girls. These sex-specific associations may be related to known differences between males and females regarding development of the lungs and lung disorders. For instance, boys are more likely to develop asthma than 
girls, whereas adult females have a higher prevalence than adult males [37], suggesting the involvement of sex hormones. Furthermore, neonate boys have a higher chance of mortality due to respiratory distress syndrome than neonate girls [38]. Next to males and females having different lung development, differences may also occur in the way male and female offspring respond to changes in maternal diet during pregnancy, as we have shown previously [39]. Future studies will have to confirm whether indeed the relation between maternal FA status and offspring lung conditions differ between male and female offspring, and if so, how these changes occur.

When interpreting results from the current study, it should be considered that the MEFAB cohort comprises a relatively healthy population. Although this consistency within the cohort enhances the comparability within participants, it also limits the applicability of the findings to less healthy populations, such as people with obesity. Furthermore, the MEFAB cohort has a relatively homogeneous genetic background, with $99 \%$ of the participants in the current analysis being Caucasian. Given the differences that exist in inflammatory markers between ethnicities $[40,41]$, the associations reported in this study may thus have been different if the ethnic background of the population would have been more diverse. Moreover, solely studying Caucasian participants in biomedical research contributes to health disparity. Future studies should therefore include other ethnicities as well, as did Rucci et al., who's Generation R cohort consisted of 66\% Europeans and 34\% non-Europeans [17].

In conclusion, our findings support the previously reported relation between inflammatory lung conditions and maternal diet during pregnancy. Furthermore, this work demonstrated the application of CCA within this field of research, which may lead to future studies using a similar approach. Lastly, future studies should investigate whether the importance of Mead acid in the relation between maternal FA status and child inflammation is due to its role as biomarker for essential fatty acid deficiency, due to its own biological function as pro-inflammatory mediator, or due to a combination of both.

\section{Acknowledgement}

The authors would like to thank all MEFAB mothers and children for their participation in the cohort. 


\section{References}

1. World Health Organization. Global Health Estimates: Life expectancy and leading causes of death and disability. Available online: https://www.who.int/data/gho/data/themes/mortality-and-global-health-estimates (accessed on 13-08-2021).

2. Centers for Disease Control and Prevention, Asthma. Available online: https://www.cdc.gov/asthma/data-visualizations/default.htm (accessed on 23-06-2021).

3. Asthma UK, Asthma prevalence. Available online: https://public.tableau.com/app/profile/asthmauk/viz/Asthmaprevalence/Asthmaprevalen ce (accessed on 23-06-2021).

4. Eurostat Data Browser, Persons reporting a chronic disease. Available online: http://appsso.eurostat.ec.europa.eu/nui/show.do?dataset=hlth_ehis_cd1e\&lang=en (accessed on 23-06-2021).

5. World Health Organization. Asthma fact sheet. Available online: https://www.who.int/news-room/fact-sheets/detail/asthma (accessed on 13-08-2021).

6. Griffiths, P.S.; Walton, C.; Samsell, L.; Perez, M.K.; Piedimonte, G. Maternal high-fat hypercaloric diet during pregnancy results in persistent metabolic and respiratory abnormalities in offspring. Pediatric research 2016, 79, 278-286.

7. MacDonald, K.D.; Moran, A.R.; Scherman, A.J.; McEvoy, C.T.; Platteau, A.S. Maternal highfat diet in mice leads to innate airway hyperresponsiveness in the adult offspring. Physiological reports 2017, 5, e13082.

8. Lumia, M.; Luukkainen, P.; Tapanainen, H.; Kaila, M.; Erkkola, M.; Uusitalo, L.; Niinistö, S.; Kenward, M.G.; Ilonen, J.; Simell, O. Dietary fatty acid composition during pregnancy and the risk of asthma in the offspring. Pediatr Allergy Immu 2011, 22, 827-835.

9. Miyake, Y.; Sasaki, S.; Tanaka, K.; Ohfuji, S.; Hirota, Y. Maternal fat consumption during pregnancy and risk of wheeze and eczema in Japanese infants aged 16-24 months: the Osaka Maternal and Child Health Study. Thorax 2009, 64, 815-821.

10. Pike, K.C.; Calder, P.C.; Inskip, H.M.; Robinson, S.M.; Roberts, G.C.; Cooper, C.; Godfrey, K.M.; Lucas, J.S. Maternal plasma phosphatidylcholine fatty acids and atopy and wheeze in the offspring at age of 6 years. Clinical and Developmental Immunology 2012, 2012.

11. Bisgaard, H.; Stokholm, J.; Chawes, B.L.; Vissing, N.H.; Bjarnadóttir, E.; Schoos, A.-M.M.; Wolsk, H.M.; Pedersen, T.M.; Vinding, R.K.; Thorsteinsdóttir, S. Fish oil-derived fatty acids in pregnancy and wheeze and asthma in offspring. New England Journal of Medicine 2016, 375, 2530-2539.

12. Hansen, S.; Strøm, M.; Maslova, E.; Dahl, R.; Hoffmann, H.J.; Rytter, D.; Bech, B.H.; Henriksen, T.B.; Granström, C.; Halldorsson, T.I. Fish oil supplementation during pregnancy and allergic respiratory disease in the adult offspring. J Allergy Clin Immun 2017, 139, 104111. e104.

13. Olsen, S.F.; Østerdal, M.L.; Salvig, J.D.; Mortensen, L.M.; Rytter, D.; Secher, N.J.; Henriksen, T.B. Fish oil intake compared with olive oil intake in late pregnancy and asthma in the offspring: 16 y of registry-based follow-up from a randomized controlled trial. The American journal of clinical nutrition 2008, 88, 167-175. 
14. Miles, E.A.; Calder, P.C. Can early omega-3 fatty acid exposure reduce risk of childhood allergic disease? Nutrients 2017, 9, 784.

15. Nwaru, B.I.; Erkkola, M.; Lumia, M.; Kronberg-Kippilä, C.; Ahonen, S.; Kaila, M.; llonen, J.; Simell, O.; Knip, M.; Veijola, R. Maternal intake of fatty acids during pregnancy and allergies in the offspring. British Journal of Nutrition 2012, 108, 720-732.

16. Notenboom, M.; Mommers, M.; Jansen, E.; Penders, J.; Thijs, C. Maternal fatty acid status in pregnancy and childhood atopic manifestations: KOALA Birth Cohort Study. Clinical \& Experimental Allergy 2011, 41, 407-416.

17. Rucci, E.; den Dekker, H.; de Jongste, J.; Steenweg-de-Graaff, J.; Gaillard, R.; Pasmans, S.; Hofman, A.; Tiemeier, H.; Jaddoe, V.; Duijts, L. Maternal fatty acid levels during pregnancy, childhood lung function and atopic diseases. The Generation R Study. Clinical \& Experimental Allergy 2016, 46, 461-471.

18. Thompson, B. A primer on the logic and use of canonical correlation analysis. Measurement and evaluation in counseling and development 1991.

19. van der Wurff, I.S.; de Groot, R.H.; Stratakis, N.; Gielen, M.; Hornstra, G.; Zeegers, M. Maastricht essential fatty acid birth cohort. Lipid Technology 2015, 27, 59-62.

20. Sherry, A.; Henson, R.K. Conducting and interpreting canonical correlation analysis in personality research: A user-friendly primer. Journal of personality assessment 2005, 84, 3748.

21. Tripathi, A.; Klami, A.; Kaski, S. Simple integrative preprocessing preserves what is shared in data sources. BMC bioinformatics 2008, 9, 1-13.

22. Naylor, M.G.; Lin, X.; Weiss, S.T.; Raby, B.A.; Lange, C. Using canonical correlation analysis to discover genetic regulatory variants. Plos One 2010, 5, e10395.

23. Al, M.D.; Van Houwelingen, A.C.; Kester, A.D.; Hasaart, T.H.; De Jong, A.E.; Hornstra, G. Maternal essential fatty acid patterns during normal pregnancy and their relationship to the neonatal essential fatty acid status. British Journal of Nutrition 1995, 74, 55-68.

24. Clauss, A. Rapid physiological coagulation method in determination of fibrinogen. Acta haematologica 1957, 17, 237-246.

25. Arab, L. Biomarkers of fat and fatty acid intake. The Journal of nutrition 2003, 133, 925S932S.

26. Ichi, I.; Kono, N.; Arita, Y.; Haga, S.; Arisawa, K.; Yamano, M.; Nagase, M.; Fujiwara, Y.; Arai, $\mathrm{H}$. Identification of genes and pathways involved in the synthesis of Mead acid (20:3n-9), an indicator of essential fatty acid deficiency. Biochimica et Biophysica Acta (BBA)-Molecular and Cell Biology of Lipids 2014, 1841, 204-213.

27. Guillou, H.; Zadravec, D.; Martin, P.G.; Jacobsson, A. The key roles of elongases and desaturases in mammalian fatty acid metabolism: Insights from transgenic mice. Progress in lipid research 2010, 49, 186-199.

28. Holman, R.T. The ratio of trienoic: tetraenoic acids in tissue lipids as a measure of essential fatty acid requirement. The Journal of nutrition 1960, 70, 405-410.

29. Patel, P.; Cossette, C.; Anumolu, J.R.; Gravel, S.; Lesimple, A.; Mamer, O.A.; Rokach, J.; Powell, W.S. Structural requirements for activation of the 5-oxo-6E, 8Z, 11Z, 14Zeicosatetraenoic acid (5-oxo-ETE) receptor: identification of a mead acid metabolite with 
potent agonist activity. Journal of Pharmacology and Experimental Therapeutics 2008, 325, 698-707.

30. Powell, W.S.; Rokach, J. Biochemistry, biology and chemistry of the 5-lipoxygenase product 5-oxo-ETE. Progress in lipid research 2005, 44, 154-183.

31. Bousquet, J.; Chanez, P.; Lacoste, J.Y.; Barnéon, G.; Ghavanian, N.; Enander, I.; Venge, P.; Ahlstedt, S.; Simony-Lafontaine, J.; Godard, P. Eosinophilic inflammation in asthma. New England Journal of Medicine 1990, 323, 1033-1039.

32. Louis, R.; Lau, L.C.; Bron, A.O.; Roldaan, A.C.; Radermecker, M.; Djukanovic, R. The relationship between airways inflammation and asthma severity. American journal of respiratory and critical care medicine 2000, 161, 9-16.

33. Shaw, D.E.; Berry, M.A.; Hargadon, B.; McKenna, S.; Shelley, M.J.; Green, R.H.; Brightling, C.E.; Wardlaw, A.J.; Pavord, I.D. Association between neutrophilic airway inflammation and airflow limitation in adults with asthma. Chest 2007, 132, 1871-1875.

34. Woodruff, P.G.; Khashayar, R.; Lazarus, S.C.; Janson, S.; Avila, P.; Boushey, H.A.; Segal, M.; Fahy, J.V. Relationship between airway inflammation, hyperresponsiveness, and obstruction in asthma. J Allergy Clin Immun 2001, 108, 753-758.

35. Wiesner, D.L.; Smith, K.D.; Kashem, S.W.; Bohjanen, P.R.; Nielsen, K. Different lymphocyte populations direct dichotomous eosinophil or neutrophil responses to pulmonary Cryptococcus infection. The Journal of Immunology 2017, 198, 1627-1637.

36. Lowe, D.M.; Redford, P.S.; Wilkinson, R.J.; O'Garra, A.; Martineau, A.R. Neutrophils in tuberculosis: friend or foe? Trends in immunology 2012, 33, 14-25.

37. Crawford, W.; Beedham, C. The changing demographic pattern in asthma related to sex and age: a study of 13,651 patients on sodium cromoglycate (Intal). Medical Journal of Australia 1976, 1, 430-434.

38. Perelman, R.H.; Palta, M.; Kirby, R.; Farrell, P.M. Discordance between male and female deaths due to the respiratory distress syndrome. Pediatrics 1986, 78, 238-242.

39. Rouschop, S.H.; Snow, S.J.; Kodavanti, U.P.; Drittij, M.-J.; Maas, L.M.; Opperhuizen, A.; van Schooten, F.J.; Remels, A.H.; Godschalk, R.W. Perinatal High-Fat Diet Influences OzoneInduced Responses on Pulmonary Oxidant Status and the Molecular Control of Mitophagy in Female Rat Offspring. International journal of molecular sciences 2021, 22, 7551.

40. Lim, E.M.; Cembrowski, G.; Cembrowski, M.; Clarke, G. Race-specific WBC and neutrophil count reference intervals. International journal of laboratory hematology 2010, 32, 590-597.

41. Paalani, M.; Lee, J.W.; Haddad, E.; Tonstad, S. Determinants of inflammatory markers in a bi-ethnic population. Ethnicity \& disease 2011, 21, 142. 


\section{Supplement}

Table S1. List of the 39 fatty acids that were measured in maternal plasma at 16, 22 and 32 weeks of pregnancy, and at day of birth. DMA - dimethyl acetal, $\operatorname{tr}$ - truncated.

\begin{tabular}{|c|c|c|c|c|c|}
\hline Saturated & $n-7$ & $n-9$ & $n-6$ & $n-3$ & Derivatives \\
\hline $14: 0$ & $16: 1 n-7$ & $18: 1 n-9$ & $18: 2 n-6$ & $18: 3 n-3$ & 18:0 DMA \\
\hline $15: 0$ & $18: 1 n-7$ & $18: 2 n-9$ & $18: 3 n-6$ & $20: 3 n-3$ & 18:1 DMA \\
\hline $16: 0$ & $20: 1 n-7$ & $20: 1 n-9$ & $20: 2 n-6$ & $20: 4 n-3$ & $16: 1 n-7$ tr \\
\hline $17: 0$ & & $20: 3 n-9$ & $20: 3 n-6$ & $20: 5 n-3$ & \\
\hline 18:0 & & $22: 1 n-9$ & $20: 4 n-6$ & $22: 3 n-3$ & \\
\hline $20: 0$ & & $22: 3 n-9$ & $22: 2 n-6$ & $22: 5 n-3$ & \\
\hline $22: 0$ & & $24: 1 n-9$ & $22: 4 n-6$ & $22: 6 n-3$ & \\
\hline 23:0 & & & $22: 5 n-6$ & & \\
\hline $24: 0$ & & & $24: 2 n-6$ & & \\
\hline
\end{tabular}




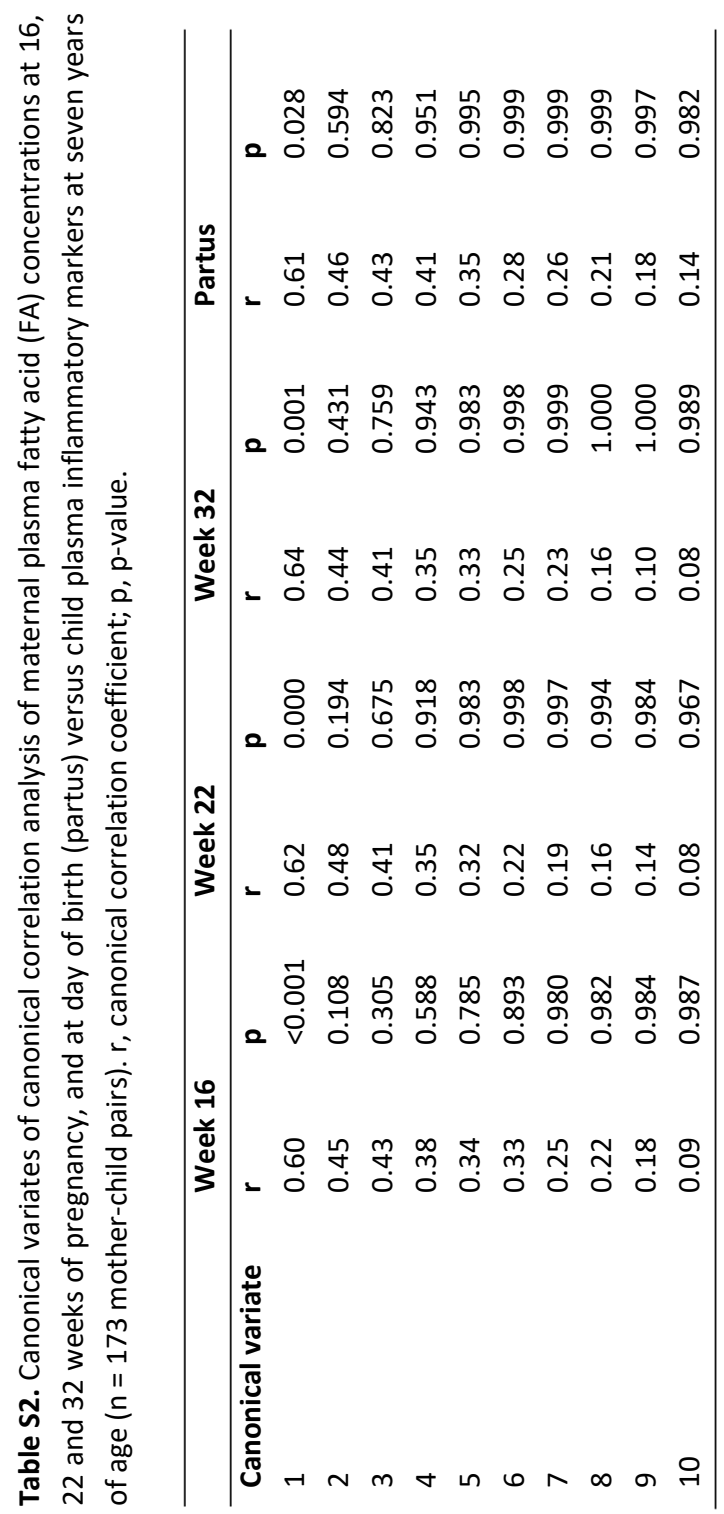


Table S3. Associations between maternal plasma mead acid concentration at day of birth and selfreported doctor diagnosis of lung conditions in the child during the first seven years of age, adjusted for various population characteristics ( $n=173$ mother-child pairs). Statistical analysis by binomial logistic regression (for asthma, bronchitis and lung infection) or ordinal logistic regression (for number of lung conditions). OR, odds ratio; $95 \% \mathrm{Cl}, 95 \%$ confidence interval.

\begin{tabular}{|c|c|c|c|}
\hline & OR & $95 \% \mathrm{Cl}$ & p-value \\
\hline \multicolumn{4}{|l|}{ Asthma } \\
\hline Unadjusted & 1.13 & $0.91-1.38$ & 0.228 \\
\hline Maternal age & 1.13 & $0.91-1.38$ & 0.241 \\
\hline Maternal BMI & 1.16 & $0.93-1.42$ & 0.166 \\
\hline Gestational age & 1.11 & $0.88-1.37$ & 0.328 \\
\hline Smoking pregnancy & 1.13 & $0.90-1.38$ & 0.242 \\
\hline Alcohol pregnancy & 1.13 & $0.90-1.37$ & 0.260 \\
\hline Birthweight & 1.13 & $0.90-1.38$ & 0.241 \\
\hline Breastfeeding & 1.15 & $0.92-1.40$ & 0.198 \\
\hline Pets & 1.13 & $0.91-1.38$ & 0.217 \\
\hline Daycare attendance & 1.13 & $0.91-1.38$ & 0.235 \\
\hline Child BMI & 1.12 & $0.90-1.37$ & 0.265 \\
\hline \multicolumn{4}{|l|}{ Lung infection } \\
\hline Unadjusted & 1.27 & $1.04-1.55$ & 0.014 \\
\hline Maternal age & 1.31 & $1.07-1.62$ & 0.009 \\
\hline Maternal BMI & 1.27 & $1.04-1.55$ & 0.016 \\
\hline Gestational age & 1.26 & $1.04-1.54$ & 0.018 \\
\hline Smoking pregnancy & 1.27 & $1.04-1.55$ & 0.014 \\
\hline Alcohol pregnancy & 1.29 & $1.06-1.58$ & 0.013 \\
\hline Birthweight & 1.27 & $1.04-1.55$ & 0.015 \\
\hline Breastfeeding & 1.26 & $1.03-1.54$ & 0.018 \\
\hline Pets & 1.27 & $1.04-1.55$ & 0.014 \\
\hline Daycare attendance & 1.27 & $1.04-1.54$ & 0.016 \\
\hline Child BMI & 1.27 & $1.04-1.54$ & 0.017 \\
\hline \multicolumn{4}{|l|}{ Bronchitis } \\
\hline Unadjusted & 1.17 & $1.01-1.34$ & 0.030 \\
\hline Maternal age & 1.17 & $1.02-1.35$ & 0.024 \\
\hline Maternal BMI & 1.18 & $1.03-1.37$ & 0.020 \\
\hline Gestational age & 1.15 & $1.00-1.32$ & 0.043 \\
\hline Smoking pregnancy & 1.19 & $1.04-1.38$ & 0.013 \\
\hline Alcohol pregnancy & 1.20 & $1.04-1.38$ & 0.013 \\
\hline Birthweight & 1.16 & $1.01-1.34$ & 0.034 \\
\hline Breastfeeding & 1.17 & $1.02-1.35$ & 0.024 \\
\hline Pets & 1.17 & $1.01-1.34$ & 0.030 \\
\hline
\end{tabular}




\begin{tabular}{llll}
\hline Daycare attendance & 1.16 & $1.01-1.34$ & 0.034 \\
Child BMI & 1.15 & $1.00-1.33$ & 0.043 \\
& & & \\
Number of lung conditions & & & \\
Unadjusted & 1.18 & $1.03-1.34$ & 0.007 \\
Maternal age & 1.19 & $1.04-1.36$ & 0.005 \\
Maternal BMI & 1.20 & $1.05-1.37$ & 0.004 \\
Gestational age & 1.17 & $1.02-1.33$ & 0.010 \\
Smoking pregnancy & 1.20 & $1.05-1.38$ & 0.003 \\
Alcohol pregnancy & 1.21 & $1.05-1.38$ & 0.003 \\
Birthweight & 1.18 & $1.03-1.34$ & 0.006 \\
Breastfeeding & 1.19 & $1.04-1.35$ & 0.006 \\
Pets & 1.18 & $1.03-1.35$ & 0.007 \\
Daycare attendance & 1.18 & $1.03-1.34$ & 0.008 \\
Child BMI & 1.17 & $1.03-1.34$ & 0.009 \\
\hline
\end{tabular}




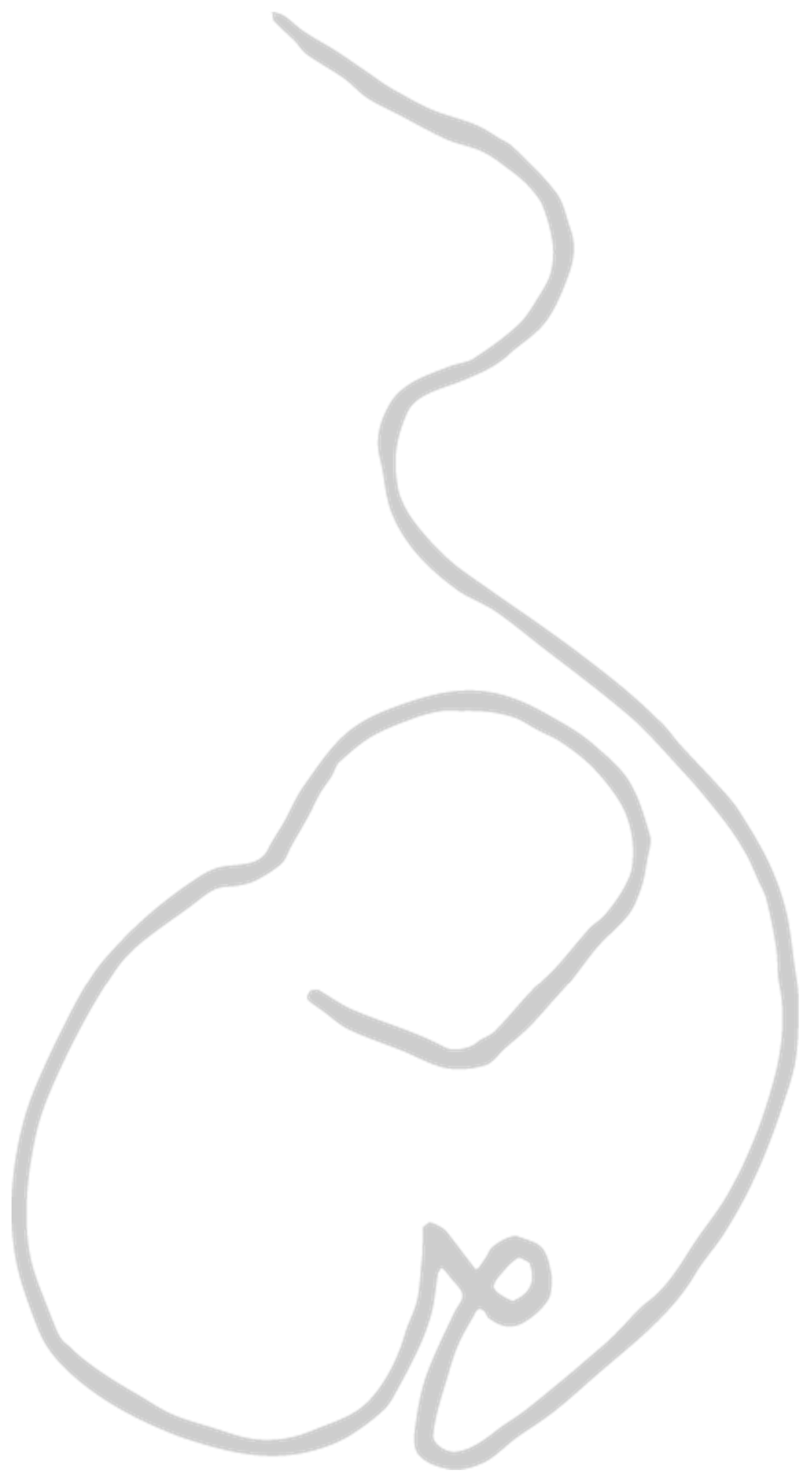

136 
Chapter 6

General discussion 


\section{Introduction}

The aim of this thesis was to study the perinatal effects of maternal dietary fat on molecular responses in offspring immunologic, metabolic and pulmonary health. In chapter $\mathbf{2}$, we showed that a perinatal high-fat diet altered offspring hepatic gene expression and DNA methylation of pathways involved in lipid metabolism, oxidative stress response, and cell proliferation. Furthermore, we demonstrated in chapter 3 that acute ozone exposure increased pulmonary oxidant status and upregulated the molecular control of mitophagy, and that in female offspring these responses were exacerbated by a perinatal high-fat diet. In chapter 4, a perinatal fish oil diet not only reduced ex vivo cytokine levels of polarizing $\mathrm{CD}^{+} \mathrm{T}$ cells, but also induced anemia with microvesicular hepatic steatosis in mouse offspring. Finally, in chapter $\mathbf{5}$, we showed that maternal fatty acid status during pregnancy was associated with child inflammatory markers at seven years of age, and that maternal Mead acid levels correlated with the development of inflammatory lung conditions in the child. In order to value the findings presented in this thesis and to extrapolate clinical implications from them, a number of topics, such as the timing of exposure, the influence of sex effects, the use of animal models, and future perspectives will be considered and evaluated in this general discussion.

\section{Timing of exposure}

The association or effect between a maternal dietary exposure and offspring health may be influenced by the timing of that specific exposure. Van Vlies et al. fed dams a high $n-3$ polyunsaturated fatty acid (PUFA) diet either during gestation, or during lactation, or during both gestation and lactation [1]. All three approaches led to an enhanced Th1-mediated immune response, as well as an attenuated Th2-mediated allergic response compared with control animals. However, the high n-3 PUFA diet was significantly more effective during lactation than during gestation alone or during gestation and lactation together. Similarly, immune cell populations in the thymus and in the spleen were significantly affected in offspring exposed to the high n-3 PUFA diet during lactation or during both gestation and lactation, compared with control, but not in offspring exposed to this diet only during gestation. These findings suggest that in mice the positive effects of supplementation with n-3 PUFAs during early life may be most beneficial during lactation, rather than during gestation. The Dutch famine studies, which examined the relation between prenatal exposure to undernutrition and health effects in adult offspring, distinguished between exposure during early, mid, or late gestation. They showed that multiple adverse health effects, such as high BMI, atherogenic lipid profile, disturbed blood coagulation profile, elevated blood pressure-response to stress, and prevalence of coronary heart disease, were all associated with famine exposure during early gestation, but not with famine exposure 
during mid or late gestation [2-6]. Conversely, prevalence of microalbuminuria and prevalence of obstructive airways diseases in adults were associated with famine exposure during mid gestation, but not with famine exposure during early or late gestation $[7,8]$. Lastly, glucose tolerance was decreased in adult offspring exposed to famine during any stage of gestation $[9,10]$. Taken together, these findings may imply that the window of greatest susceptibility not only depends on the specific exposure, but also on the associated health outcome.

In this thesis, the influence of the timing of exposure was examined in chapter $\mathbf{5}$. In this chapter, we showed that even without an intervention, a prenatal exposure may vary over time, as was indicated by the gradual change in maternal plasma fatty acid profile throughout pregnancy in mothers of the MEFAB cohort. Despite these differences in exposure between the prenatal time points (i.e. weeks 16, 22 and 32 of pregnancy, and day of birth), significant multivariate correlations were found between maternal plasma fatty acid status and offspring inflammatory health for all examined prenatal time points. In chapter $\mathbf{2}$ and chapter $\mathbf{3}$ of this thesis, dams were fed a high-fat diet starting from six weeks before gestation until the end of lactation. In chapter 4 , dams received a fish oil diet during gestation and lactation. For these three chapters, the effects of a different window of exposure were not studied. However, considering the influence timing of exposure is known to have on the relation between maternal dietary exposures and offspring health, findings in these chapters may thus have been different if other windows of exposure would have been applied. For future intervention studies, researchers should not only contemplate the perinatal period during which mothers or dams are exposed, but also take the specific timing of exposure into account when comparing their results with literature.

\section{Sex effects}

Next to timing of exposure, offspring sex may influence the association or effect between maternal dietary exposure and offspring health. Ornellas et al. showed that a maternal highfat diet in mice had various detrimental effects on metabolic health in both male and female offspring. However, effects on feed intake, body weight gain, fasting glucose levels, glucose intolerance, plasma triglycerides and plasma insulin were largest in males, whereas females were more affected in terms of adiposity [11]. Snow et al. fed female rats a high-fat diet before mating and during gestation and lactation to examine the effect of a perinatal highfat diet on ozone susceptibility in offspring [12]. They found that the ozone exposure elicited greater metabolic impact in female than male offspring, and that the maternal high-fat diet further potentiated this effect in female offspring. 
In this thesis, the influence of offspring sex on the relation between maternal diet and offspring health was studied in chapter $\mathbf{3}$, chapter 4 and chapter 5 . In chapter $\mathbf{3}$, acute ozone exposure increased pulmonary oxidative stress and upregulated the molecular machinery that controls receptor-mediated mitophagy. In female offspring, the perinatal high-fat diet exacerbated these responses, whereas in males, responses were similar between offspring from control diet dams and offspring from high-fat diet dams. In chapter $\mathbf{5}$, the association between maternal mead acid plasma levels at day of birth and the development of lung conditions in offspring was affected by offspring sex as well. After stratification for sex, associations between maternal Mead acid levels and asthma, bronchitis and the total number of lung conditions was significant for girls, but not boys, whereas for lung infection, the association was only significant for boys, but not girls. In chapter 4, a maternal fish oil diet affected ex vivo polarization of naïve $\mathrm{CD}^{+} \mathrm{T}$ cells isolated from offspring spleen. For this study, both male and female offspring were included and statistical analysis were adjusted for offspring sex. However, none of the measured outcomes in offspring showed a significant effect of sex. This may have been because offspring animals were assessed at such a young age that hormonal differences between both sexes were insufficiently developed to induce sex effects [13]. In chapter $\mathbf{2}$, sex effects were not assessed, since only male offspring was included in the experiment. Major contributors to sex dimorphisms are estrogens and androgens [14-17]. In females, these effects depend on the hormonal cycle, making females more prone to variation in specific outcomes. For this reason, often only males are included in biomedical studies. However, for scientific results to be properly translated into clinical practice, future studies should not only include both sexes in studies, but also assess outcomes for both sexes separately, or at least adjust for sex in statistical analyses.

\section{Animal models - Ethics}

Many studies use animal models to examine the effects of prenatal diets on offspring health. Major advantages of working with small animals such as rats and mice are their short gestation time and lifespan, relatively inexpensive maintenance costs, control over environmental variability, and the ease of diet manipulation [18]. In the Netherlands, performing an animal experiment is prohibited, unless it can be justified that 1 ) the scientific gain of the experiment outweighs the harm caused to the animal; and that 2) the research question cannot be answered with any alternative approach. Whether or not an animal study meets these requirements is assessed by the Central Committee Animal Experiments (Centrale Commissie Dierproeven, CCD), which is advised by the Animal Experiment Committee (Dierexperimentencommissie, DEC). 
When applying for approval of the study described in chapter 4, the Animal Experiment Committee initially judged that both conditions for approval of an animal experiment were not met. For the first condition, the committee based its point of view on the fact that the study revolved around the polarization of $\mathrm{CD}^{+} \mathrm{T}$ cells and its role in the anti-atopic effects of maternal supplementation with n-3 PUFAs. The committee argued that atopic diseases are not lethal and that therefore this research was not relevant enough to justify the use of animals. For the second condition, the committee did agree that studying prenatal exposures is impossible in vitro. However, they did not see any ethical restrictions to performing the study in humans. In a rebuttal, we challenged the committee's decision on both conditions. For the first condition, we discussed that although this specific study revolved around atopic diseases, the polarization of $\mathrm{CD}^{+} \mathrm{T}$ cells is a fundamental physiological process within the adaptive immune system which can be applied to many (also lethal) diseases. In addition, we believe that preventing the development of a disease that significantly affects a patients quality of life is sufficiently relevant to justify the use of animals, even more since an increasing number of diseases that was previously lethal, is chronic nowadays due to improved treatments (e.g. AIDS). For the second condition, we argued that to perform this research in humans, the study should comply with the Embryo Law, since any intervention applied to a pregnant female also affects the unborn child. The Embryo Law states that research involving an embryo is prohibited, unless 1 ) the research contributes to diagnostics, prevention or treatment of a severe condition in that specific embryo that is subjected to the treatment; or 2) the objections and risks of the intervention have negligible objections and risks for the embryo. For our study, this first condition would not be met, since the intervention would have been applied to healthy women without any indication for future risk of the development of atopy in their children, which made this a fundamental study. Likewise, this second condition was not met as well. To assess the polarization of naïve $\mathrm{CD}^{+} \mathrm{T}$ cells, these cells would have to be isolated from secondary lymphoid organs (such as lymph nodes or the spleen) or from peripheral blood. The inability to access lymphoid organs in a human requires no further clarification. Furthermore, withdrawing a sufficient amount of blood for isolation of naïve $\mathrm{CD} 4^{+} \mathrm{T}$ cells is such a negative experience for a one-year-old child (sensitization to allergens is thought to occur early in life [19]), that the objections and risks would not be negligible. Hence, assessing the research question in humans would be unethical and thus would not be approved. In the end, the committee reconsidered its opinion and granted approval for the animal model as described in chapter 4 .

Retrospectively, the maternal fish oil diet as studied in chapter 4 turned out to have serious adverse effects. The perinatal fish oil diet induced anemia in both dams and offspring, and caused microvesicular hepatic steatosis in offspring. In the discussion section of chapter 4, we discuss that this may be due to the dosage of $n-3$ PUFAs, which was ten times higher in 
our study than in previous clinical studies. Nevertheless, our study demonstrated that a perinatal fish oil diet is able to induce detrimental effects in both mothers and offspring, and thus that future studies, both animal and clinical, should carefully consider their dosage. Besides chapter 4 , chapters 2 and $\mathbf{3}$ used animal models as well to study the effect of maternal diet on offspring health. For these chapters, the design and execution of the animal model were performed by collaborating research groups, which kindly provided samples in order for us to answer research questions that continued on previous findings of those collaborators $[12,20,21]$. This strategy prevents the unnecessary use of animals that would otherwise occur if a new experiment was performed instead of reusing samples for subsequent studies.

Altogether, we believe that the advantages and scientific gains obtained from using animal models outweigh the disadvantages related to animal welfare and translation, as long as the balance between these benefits and drawbacks is carefully assessed per individual case by external committees. Nevertheless, researchers should be aware of the differences between model species and humans, and of the implications hereof for translation of findings from animals models to clinical practice.

\section{Animal models - Litter effects}

One major challenge in studying effects of maternal diet on offspring health in animals is the use of multiparous species, such as rats and mice [22]. When studying maternal exposures using animal models, the treatment is applied to pregnant females and thus to all unborn offspring animals within that female. Consequently, the litter is the experimental unit, as this is the smallest unit to which a treatment can be randomly assigned. The outcomes, however, are measured in individual offspring animals, since these are of scientific interest. Subsequently assessing each individual offspring animal as a separate experimental unit leads to sample size inflation and would be similar to applying a treatment to a single patient and then measuring its blood pressure in both arms and assuming this represents an $n$ of 2 . In addition to this, animals from the same liter are more alike than animals from different litters, causing litter mates to be correlated. However, routine analysis methods such as t-test, ANOVA and linear regression assume individuals to be independent of each other. Ignoring the dependencies that actually exist within animals from the same litter may lead to increased false-positive rate or low power [23]. To prevent this, several experimental approaches can be applied [22]. The simplest option is to randomly select one offspring animal per litter and measure the outcome on that animal. Alternatively, multiple offspring animals per litter can be assessed, after which values are averaged for each litter. Lastly, multiple animals can be measured per litter, after which 
statistical analysis is performed using mixed-effects models, which are able to take into account the correlated data structure.

In this thesis, litter effects were handled with different approaches. In chapter 3, dams were fed a high-fat diet or a control diet before mating, and during gestation and lactation, to study the effect of a perinatal high-fat diet on ozone susceptibility in offspring. Before offspring was exposed to ozone, four pups per litter were randomly selected and allocated to an exposure group: one female for air exposure, one female for ozone exposure, one male for air exposure, and one male for ozone exposure. By spreading animals from the same litter over different experimental groups, observations were independent, and routine approaches (in this case ANOVA) could be used for statistical analysis. In chapter 4, dams received a fish oil diet or a control diet during gestation and lactation to examine the effect of perinatal $n-3$ PUFAs on offspring CD4 ${ }^{+} T$ cells. Outcomes were assessed in all offspring animals, after which data were analyzed with linear mixed-effects models. Advantages of this approach are that no animals are wasted, and that less dams have to be included in the experiment to reach a sufficient sample size compared with when only one offspring per litter is analyzed or when outcomes are averaged per litter. In chapter $\mathbf{5}$, litter effects were not an issue, since only singletons were included in the MEFAB cohort. The only chapter in this thesis for which litter effects were handled suboptimally, is chapter $\mathbf{2}$. In this chapter, dams were fed a high-fat diet or a control diet before mating, and during gestation and lactation. After this, offspring animals were divided over two groups. The first group was assessed at 12 weeks of age, whereas the other was assessed at 28 weeks of age. For the first group, only one animal per litter was selected, thereby preventing issues related to litter effects. For the second group, however, 11 animals were independent (they originated from 11 different litters), whereas the other 7 animals originated from 3 litters. Instead of handling the dependency of observations within this group by analyzing data with mixed-effects models, data were analyzed with a t-test. Although in this particular case the pseudoreplication was minimal compared with an experiment that would have used all litter mates, results may still have been biased to some extent. For future studies, to ensure correct experimental design and statistical analysis of animal models using multiparous species, researchers should be aware of the potential pitfalls these experiments harbor. Herein lies an important role for authorities that review animal experiments, such as the Animal Welfare Body and Animal Experiment Committee. All animal experiments require approval of these authorities, providing the opportunity to prevent issues arising from litter effects when studies are still in their design phase. 


\section{Clinical and public health implications}

Maternal consumption of a high-fat diet and obesity during pregnancy and lactation predisposes offspring to developing various metabolic disorders, such as obesity and type 2 diabetes, and cardiovascular diseases [24-27]. In chapter 2, a perinatal high-fat diet altered hepatic DNA methylation and gene expression of pathways related to lipid synthesis and uptake, oxidative stress response, and cell proliferation. These changes were mechanistically linked to previously reported phenotypic effects of maternal high-fat diet, such as increases in body weight and fat mass, and induction of non-alcoholic fatty liver disease $[11,28,29]$. These findings provide evidence for the involvement of epigenetics and regulation of gene expression in the effects of prenatal obesity and high-fat diet on offspring metabolic health.

In chapter 3, a perinatal high-fat diet exacerbated ozone-induced increases in pulmonary oxidant status and upregulation of the molecular control of mitophagy. These outcomes suggest that mitochondria and oxidative stress play a role in the effect of maternal diet on ozone susceptibility in offspring. Taken together, the results from chapters $\mathbf{2}$ and $\mathbf{3}$ further necessitate the investment in early life nutrition to protect offspring from detrimental metabolic and pulmonary effects.

Clinical studies have previously shown that supplementation with $\mathrm{n}-3$ PUFAs during pregnancy may reduce the risk of developing asthma in children [30-32]. In chapter 4, we showed that a perinatal fish oil diet, which contains high amounts of $n-3$ PUFAs, decreased extracellular cytokine levels of polarizing $\mathrm{CD} 4^{+} \mathrm{T}$ cells. Polarization of $\mathrm{CD} 4^{+} \mathrm{T}$ cells plays an important role in the development of asthma and other atopic diseases [33], and our findings may thus provide evidence for a mechanistic link between maternal $n-3$ PUFAs and development of atopy in offspring. This mechanistic link, in combination with evidence from observational and clinical studies, corroborates the need for a dietary advise on prenatal consumption and supplementation of n-3 PUFAs. In chapter 5, we demonstrated that maternal fatty acid status correlated with child inflammation at seven years of age, and that maternal Mead acid levels were associated with development of inflammatory lung conditions. Considering that Mead acid is a biomarker for essential fatty acid deficiency [34], that Mead acid levels increased throughout pregnancy (chapter 5), and that Mead acid metabolites have pro-inflammatory effects [35], these findings further substantiate the need for dietary advise on consumption and supplementation of fatty acids during pregnancy. However, besides positive effects on $\mathrm{CD}^{+} \mathrm{T}$ cell polarization, the perinatal fish oil diet in chapter 4 also induced anemia in both dams and offspring, and caused hepatic microvesicular steatosis in offspring. These findings stress the need to further research potential adverse effects of prenatal supplementation with high levels of $n-3$ PUFAs on maternal and offspring health. If institutions like the Health Council (Gezondheidsraad) or 
the Nutrition Centre (Voedingscentrum) would come to publish an advise for prenatal supplementation with n-3 PUFAs to protect children against asthma or other atopic conditions, upper dosage limits should be carefully considered to prevent adverse effects.

\section{Future perspectives}

Based on the topics discussed so far, a number of recommendations may be done for studies yet to come. Firstly, studies that examine specific maternal exposures should compare the effects of different windows of exposure on offspring health. Many animal studies apply an intervention throughout gestation and lactation to ensure that any window of opportunity is not missed. However, by sticking to this approach, it will never be learned which exposure or intervention should be prevented or applied, respectively, during which perinatal phase in order to improve offspring health. Next to this, it would be beneficial if animal and human studies would not only include both sexes, but also analyze them separately. This would improve the translation to new clinical studies and prevent further health disparity between men and women. In addition, future animal studies using multiparous species should take litter effects into account, to prevent the unnecessary introduction of false-positive or false-negative effects, thereby improving the translatability of animal results. Next to these general recommendations, future studies may follow up on findings presented in this thesis. For instance, future studies may examine whether adverse effects of a maternal high-fat diet on offspring metabolism are regulated by changes in offspring gene expression and DNA methylation, as was suggested by the findings from chapter 2. Furthermore, the involvement of oxidative stress and mitochondrial function in the effects of maternal high-fat diet on ozone susceptibility in offspring may be further studied. In addition, future studies should find out whether the adverse effects reported in chapter 4 were indeed caused by high levels of $n-3$ PUFAs and how these detrimental effects can be prevented in future studies. Lastly, it may be examined whether Mead acid is mechanistically involved in the relation between maternal fatty acid status and inflammatory lung diseases in the child.

\section{Conclusions}

The aim of this thesis was to study the perinatal effects of maternal dietary fat on molecular responses in offspring immunologic, metabolic and pulmonary health. In chapter $\mathbf{2}$, we showed that a perinatal high-fat diet altered offspring hepatic gene expression and DNA methylation of pathways involved in lipid metabolism, oxidative stress response, and cell proliferation. Furthermore, we demonstrated in chapter 3 that acute ozone exposure increased pulmonary oxidant status and upregulated the molecular control of mitophagy, and that in female offspring these responses were exacerbated by a perinatal high-fat diet. 
In chapter 4, a perinatal fish oil diet not only reduced ex vivo cytokine levels of polarizing $\mathrm{CD}^{+} \mathrm{T}$ cells, but also induced anemia with microvesicular hepatic steatosis in mouse offspring. In addition, we showed in chapter $\mathbf{5}$ that maternal fatty acid status during pregnancy was associated with child inflammation at seven years of age, and that maternal Mead acid levels correlated with the development of inflammatory lung conditions in the child. Finally, in chapter 6 , the general discussion, we discussed that the association between maternal dietary exposure and offspring health may be influenced not only by the timing of that specific exposure, but also by the sex of the offspring. Next to this, we discussed ethical and practical issues that need to be considered when using animal models for studying the effects of maternal diet on offspring health.

Altogether, this thesis has shown the influence of maternal dietary fat on molecular responses in offspring immunologic, metabolic and pulmonary health. Through these findings, this thesis has further substantiated the importance of maternal diet for later-life health in offspring. Future research should continue to examine mechanisms that underlie both adverse and beneficial effects of maternal nutrition, to further improve the health of future generations. 


\section{References}

1. Van Vlies, N.; Hogenkamp, A.; Fear, A.; Van Esch, B.; Oosting, A.; Van de Heijning, B.; Van Der Beek, E.; Calder, P.; Garssen, J. Perinatal programming of murine immune responses by polyunsaturated fatty acids. Journal of developmental origins of health and disease 2011, 2, 112-123.

2. Roseboom, T.J.; van der Meulen, J.H.; Osmond, C.; Barker, D.J.; Ravelli, A.C.; Bleker, O.P. Plasma lipid profiles in adults after prenatal exposure to the Dutch famine. The American journal of clinical nutrition 2000, 72, 1101-1106.

3. Roseboom, T.J.; Van Der Meulen, J.H.; Ravelli, A.C.; Osmond, C.; Barker, D.J.; Bleker, O.P. Plasma fibrinogen and factor VII concentrations in adults after prenatal exposure to famine. British journal of haematology 2000, 111, 112-117.

4. Ravelli, A.C.; Van Der Meulen, J.H.; Osmond, C.; Barker, D.J.; Bleker, O.P. Obesity at the age of $50 \mathrm{y}$ in men and women exposed to famine prenatally. The American journal of clinical nutrition 1999, 70, 811-816.

5. Painter, R.C.; de Rooij, S.R.; Bossuyt, P.M.; Phillips, D.I.; Osmond, C.; Barker, D.J.; Bleker, O.P.; Roseboom, T.J. Blood pressure response to psychological stressors in adults after prenatal exposure to the Dutch famine. Journal of hypertension 2006, 24, 1771-1778.

6. Roseboom, T.J.; van der Meulen, J.H.; Osmond, C.; Barker, D.J.; Ravelli, A.C.; SchroederTanka, J.M.; van Montfrans, G.A.; Michels, R.P.; Bleker, O.P. Coronary heart disease after prenatal exposure to the Dutch famine, 1944-45. Heart 2000, 84, 595-598.

7. Lopuhaä, C.; Roseboom, T.; Osmond, C.; Barker, D.; Ravelli, A.; Bleker, O.; van Der Zee, J.; Van Der Meulen, J. Atopy, lung function, and obstructive airways disease after prenatal exposure to famine. Thorax 2000, 55, 555-561.

8. Painter, R.C.; Roseboom, T.J.; Van Montfrans, G.A.; Bossuyt, P.M.; Krediet, R.T.; Osmond, C.; Barker, D.J.; Bleker, O.P. Microalbuminuria in adults after prenatal exposure to the Dutch famine. Journal of the American Society of Nephrology 2005, 16, 189-194.

9. de Rooij, S.R.; Painter, R.C.; Roseboom, T.J.; Phillips, D.; Osmond, C.; Barker, D.; Tanck, M.; Michels, R.; Bossuyt, P.; Bleker, O. Glucose tolerance at age 58 and the decline of glucose tolerance in comparison with age 50 in people prenatally exposed to the Dutch famine. Diabetologia 2006, 49, 637-643.

10. Ravelli, A.C.; van der Meulen, J.H.; Michels, R.; Osmond, C.; Barker, D.J.; Hales, C.; Bleker, O.P. Glucose tolerance in adults after prenatal exposure to famine. The Lancet 1998, 351, 173-177.

11. Ornellas, F.; Souza-Mello, V.; Mandarim-de-Lacerda, C.A.; Aguila, M.B. Programming of obesity and comorbidities in the progeny: lessons from a model of diet-induced obese parents. Plos One 2015, 10, e0124737.

12. Snow, S.J.; Broniowska, K.; Karoly, E.D.; Henriquez, A.R.; Phillips, P.M.; Ledbetter, A.D.; Schladweiler, M.C.; Miller, C.N.; Gordon, C.J.; Kodavanti, U.P. Offspring susceptibility to metabolic alterations due to maternal high-fat diet and the impact of inhaled ozone used as a stressor. Scientific reports 2020, 10, 1-18.

13. Bell, M.R. Comparing postnatal development of gonadal hormones and associated social behaviors in rats, mice, and humans. Endocrinology 2018, 159, 2596-2613. 
14. Mauvais-Jarvis, F.; Clegg, D.J.; Hevener, A.L. The role of estrogens in control of energy balance and glucose homeostasis. Endocrine reviews 2013, 34, 309-338.

15. Navarro, G.; Allard, C.; Xu, W.; Mauvais-Jarvis, F. The role of androgens in metabolism, obesity, and diabetes in males and females. Obesity 2015, 23, 713-719.

16. Fuentes, N.; Nicoleau, M.; Cabello, N.; Montes, D.; Zomorodi, N.; Chroneos, Z.C.; Silveyra, P. $17 \beta$-Estradiol affects lung function and inflammation following ozone exposure in a sexspecific manner. American Journal of Physiology-Lung Cellular and Molecular Physiology 2019, 317, L702-L716.

17. Taneja, V. Sex hormones determine immune response. Frontiers in immunology 2018, 9, 1931.

18. McMullen, S.; Mostyn, A. Animal models for the study of the developmental origins of health and disease: Workshop on 'Nutritional models of the developmental origins of adult health and disease'. Proceedings of the Nutrition Society 2009, 68, 306-320.

19. Holt, P.; Naspitz, C.; Warner, J.O. Early immunological influences. Prevention of Allergy and Allergic Asthma 2004, 84, 102-127.

20. Snow, S.J.; Phillips, P.M.; Ledbetter, A.; Johnstone, A.F.; Schladweiler, M.C.; Gordon, C.J.; Kodavanti, U.P. The influence of maternal and perinatal high-fat diet on ozone-induced pulmonary responses in offspring. Journal of Toxicology and Environmental Health, Part $A$ 2019, 82, 86-98.

21. van Ewijk, P.A.; Paglialunga, S.; Kooi, M.E.; Nunes, P.M.; Gemmink, A.; Slenter, J.; Kornips, E.; Jorgensen, J.A.; Hoeks, J.; Wildberger, J.E.; et al. Effects of high-fat feeding on ectopic fat storage and postprandial lipid metabolism in mouse offspring. Obesity (Silver Spring) 2015, 23, 2242-2250, doi:10.1002/oby.21235.

22. Lazic, S.E.; Essioux, L. Improving basic and translational science by accounting for litter-tolitter variation in animal models. BMC neuroscience 2013, 14, 1-11.

23. Holson, R.; Pearce, B. Principles and pitfalls in the analysis of prenatal treatment effects in multiparous species. Neurotoxicology and teratology 1992, 14, 221-228.

24. Eriksson, J.G.; Sandboge, S.; Salonen, M.K.; Kajantie, E.; Osmond, C. Long-term consequences of maternal overweight in pregnancy on offspring later health: findings from the Helsinki Birth Cohort Study. Annals of medicine 2014, 46, 434-438.

25. Gaillard, R.; Steegers, E.A.; Duijts, L.; Felix, J.F.; Hofman, A.; Franco, O.H.; Jaddoe, V.W. Childhood cardiometabolic outcomes of maternal obesity during pregnancy: the Generation R Study. Hypertension 2014, 63, 683-691.

26. Hochner, H.; Friedlander, Y.; Calderon-Margalit, R.; Meiner, V.; Sagy, Y.; Avgil-Tsadok, M.; Burger, A.; Savitsky, B.; Siscovick, D.S.; Manor, O. Associations of Maternal Prepregnancy Body Mass Index and Gestational Weight Gain With Adult Offspring Cardiometabolic Risk FactorsClinical Perspective: The Jerusalem Perinatal Family Follow-Up Study. Circulation 2012, 125, 1381-1389.

27. Yu, Z.; Han, S.; Zhu, J.; Sun, X.; Ji, C.; Guo, X. Pre-pregnancy body mass index in relation to infant birth weight and offspring overweight/obesity: a systematic review and metaanalysis. Plos One 2013, 8, e61627. 
28. Keleher, M.R.; Zaidi, R.; Shah, S.; Oakley, M.E.; Pavlatos, C.; El Idrissi, S.; Xing, X.; Li, D.; Wang, T.; Cheverud, J.M. Maternal high-fat diet associated with altered gene expression, DNA methylation, and obesity risk in mouse offspring. Plos One 2018, 13, e0192606.

29. Pruis, M.; Lendvai, A.; Bloks, V.; Zwier, M.; Baller, J.; De Bruin, A.; Groen, A.; Plösch, T. Maternal western diet primes non-alcoholic fatty liver disease in adult mouse offspring. Acta Physiologica 2014, 210, 215-227.

30. Bisgaard, H.; Stokholm, J.; Chawes, B.L.; Vissing, N.H.; Bjarnadóttir, E.; Schoos, A.-M.M.; Wolsk, H.M.; Pedersen, T.M.; Vinding, R.K.; Thorsteinsdóttir, S. Fish oil-derived fatty acids in pregnancy and wheeze and asthma in offspring. New England Journal of Medicine 2016, 375, 2530-2539.

31. Hansen, S.; Strøm, M.; Maslova, E.; Dahl, R.; Hoffmann, H.J.; Rytter, D.; Bech, B.H.; Henriksen, T.B.; Granström, C.; Halldorsson, T.I. Fish oil supplementation during pregnancy and allergic respiratory disease in the adult offspring. J Allergy Clin Immun 2017, 139, 104111. e104.

32. Olsen, S.F.; Østerdal, M.L.; Salvig, J.D.; Mortensen, L.M.; Rytter, D.; Secher, N.J.; Henriksen, T.B. Fish oil intake compared with olive oil intake in late pregnancy and asthma in the offspring: 16 y of registry-based follow-up from a randomized controlled trial. The American journal of clinical nutrition 2008, 88, 167-175.

33. Yoo, Y.; Perzanowski, M.S. Allergic sensitization and the environment: latest update. Current allergy and asthma reports 2014, 14, 465.

34. Holman, R.T. The ratio of trienoic: tetraenoic acids in tissue lipids as a measure of essential fatty acid requirement. The Journal of nutrition 1960, 70, 405-410.

35. Patel, P.; Cossette, C.; Anumolu, J.R.; Gravel, S.; Lesimple, A.; Mamer, O.A.; Rokach, J.; Powell, W.S. Structural requirements for activation of the 5-oxo-6E, 8Z, 11Z, 14Zeicosatetraenoic acid (5-oxo-ETE) receptor: identification of a mead acid metabolite with potent agonist activity. Journal of Pharmacology and Experimental Therapeutics 2008, 325, 698-707. 


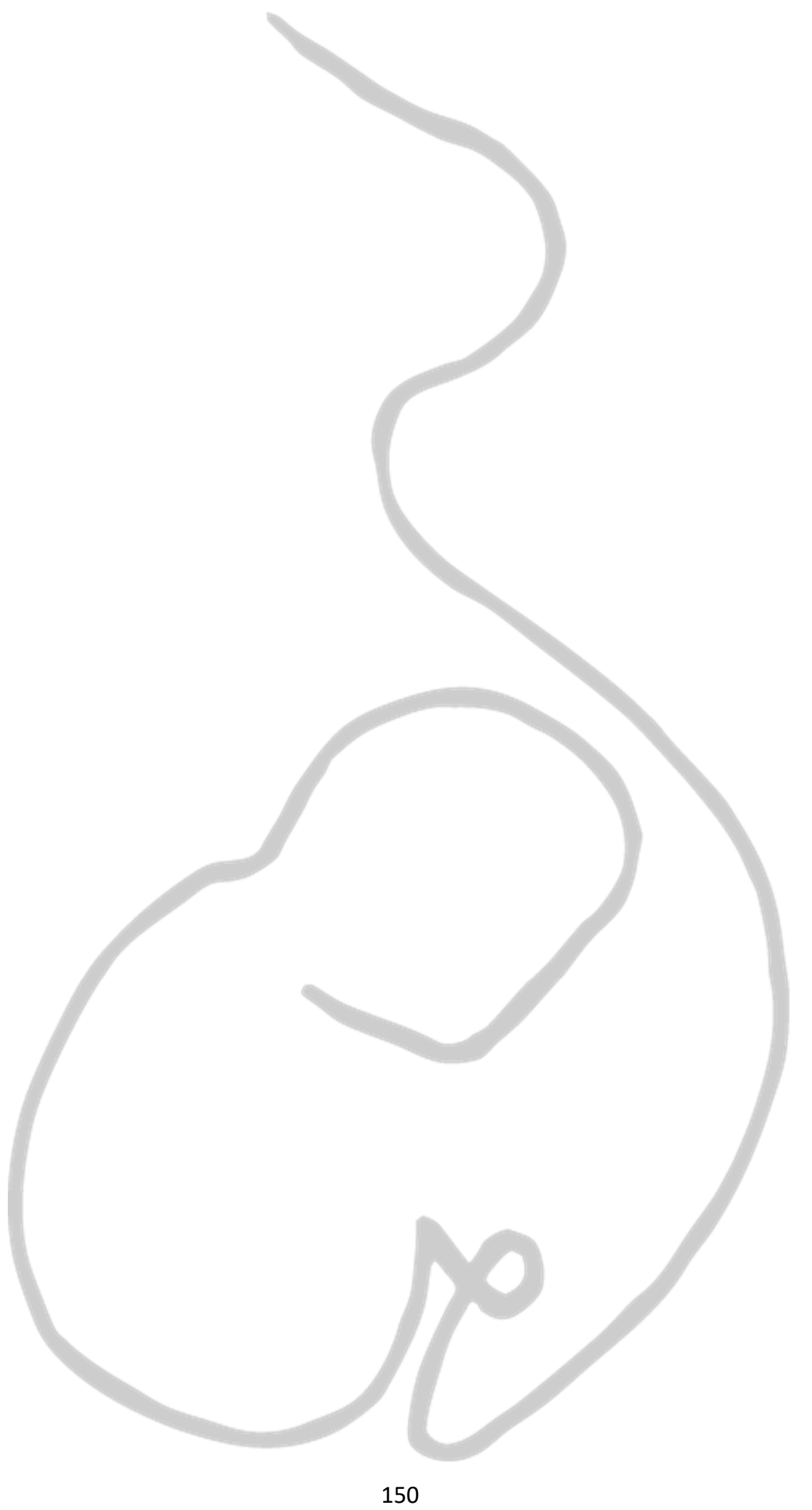


Summary 


\section{Summary}

The fat content and fatty acid composition of the maternal diet during pregnancy and lactation greatly influence the offspring's immunologic, metabolic and pulmonary health. However, the molecular responses and mechanisms that are involved in these effects remain largely unknown. Therefore, this thesis aimed to study the effects of maternal dietary fatty acids and high-fat diet on the molecular responses related to offspring immunologic, metabolic and pulmonary health.

Exposure to a perinatal high-fat diet leads to an impaired metabolic phenotype in mouse offspring. It is less well-known, however, through which mechanisms these long-term effects are transmitted. Therefore, we studied the effect of a combined paternal and maternal high-fat diet on hepatic gene expression and DNA methylation in adult mouse offspring. In chapter 2, we showed that this perinatal high-fat diet altered hepatic expression of gene sets involved in lipid metabolism, oxidative stress response and cell proliferation. Furthermore, the perinatal high-fat increased hepatic oxidative stress. In addition, transcription factor binding sites upstream of the metabolic gene Lpin1 were hypermethylated in offspring livers in response to the perinatal high-fat diet. We hypothesize that these changes underlie the previously reported metabolic disturbances in offspring after exposure to a prenatal high-fat diet.

Next to affecting offspring metabolism, a perinatal high-fat diet is also able to exacerbate ozone-induced adverse effects on lung function, injury and inflammation in offspring, and it has been suggested that mitochondrial dysfunction is involved in this effect. Therefore, the aim of chapter $\mathbf{3}$ was to investigate whether a perinatal obesogenic high-fat diet affects ozone-induced changes in offspring pulmonary oxidant status and the molecular control of mitochondrial function. In this study, acute ozone exposure increased pulmonary oxidant status and upregulated the molecular machinery that controls receptor-mediated mitophagy. In female offspring, a perinatal high-fat diet exacerbated these responses, whereas in male offspring, responses were similar for both diet groups. These findings suggest that a perinatal high-fat influences ozone-induced responses on pulmonary oxidant status and the molecular control of mitophagy in female rat offspring.

Maternal supplementation with n-3 polyunsaturated fatty acids (PUFAs) during pregnancy is associated with reductions of asthma features in offspring. The underlying mechanisms, however, are unclear. Since a skewed polarization of $\mathrm{CD} 4^{+} \mathrm{T}$ cells is involved in asthmatic development, we studied the effect of a perinatal n-3 PUFA-rich fish oil diet on ex vivo polarization of $\mathrm{CD} 4^{+} \mathrm{T}$ cells in mouse offspring. In chapter 4 , we showed that a perinatal fish oil diet reduced extracellular cytokine levels of both Th1- and Th2-polarizing CD4+ $\mathrm{T}$ cells. In 
addition, we found that a perinatal fish oil diet induced not only anemia in both dams and offspring, but also in microvesicular steatosis in offspring liver. These results indicate that perinatal exposure to high levels of $n-3$ PUFAs may have both beneficial and adverse effects in the offspring and thus that dosages should be carefully considered for future studies.

The development of inflammatory lung disorders has previously been shown to be related to maternal diet during pregnancy. However, estimates of maternal fatty acid status and statistical analysis were often suboptimal in previous studies. Therefore, we examined in chapter $\mathbf{5}$ the relation between maternal fatty status and child inflammation by analyzing data from the MEFAB cohort using canonical correlation analysis (CCA), a multivariate statistical model. This chapter showed that maternal fatty acid status during pregnancy was significantly associated with child inflammation at seven years of age and that the fatty acid Mead acid was most important for this correlation. In addition, maternal Mead acid levels at day of birth were significantly correlated with the development of inflammatory lung conditions in the child. Future studies should investigate whether the importance of Mead acid in the relation between maternal fatty acid status and child inflammation is due to its role as biomarker for essential fatty acid deficiency, due to its own biological function as pro-inflammatory mediator, or possibly a combination of both.

In summary, this thesis aimed to study the effects of maternal dietary fatty acids and highfat diet on the molecular responses related to offspring immunologic, metabolic and pulmonary health. We showed that a maternal high-fat diet is able to affect gene expression and DNA methylation in offspring liver, and to alter ozone-induced pulmonary responses in female offspring. Furthermore, we demonstrated that a maternal fish oil diet has positive effects on ex vivo polarization of offspring $C D 4^{+} \mathrm{T}$ cells, but also adverse effects on maternal and offspring health. Lastly, we showed that maternal fatty acid status during pregnancy is associated with offspring inflammation and development of inflammatory lung conditions. These findings aid to better understand how maternal nutrition affects offspring health and may contribute to improve the health of future generations. 


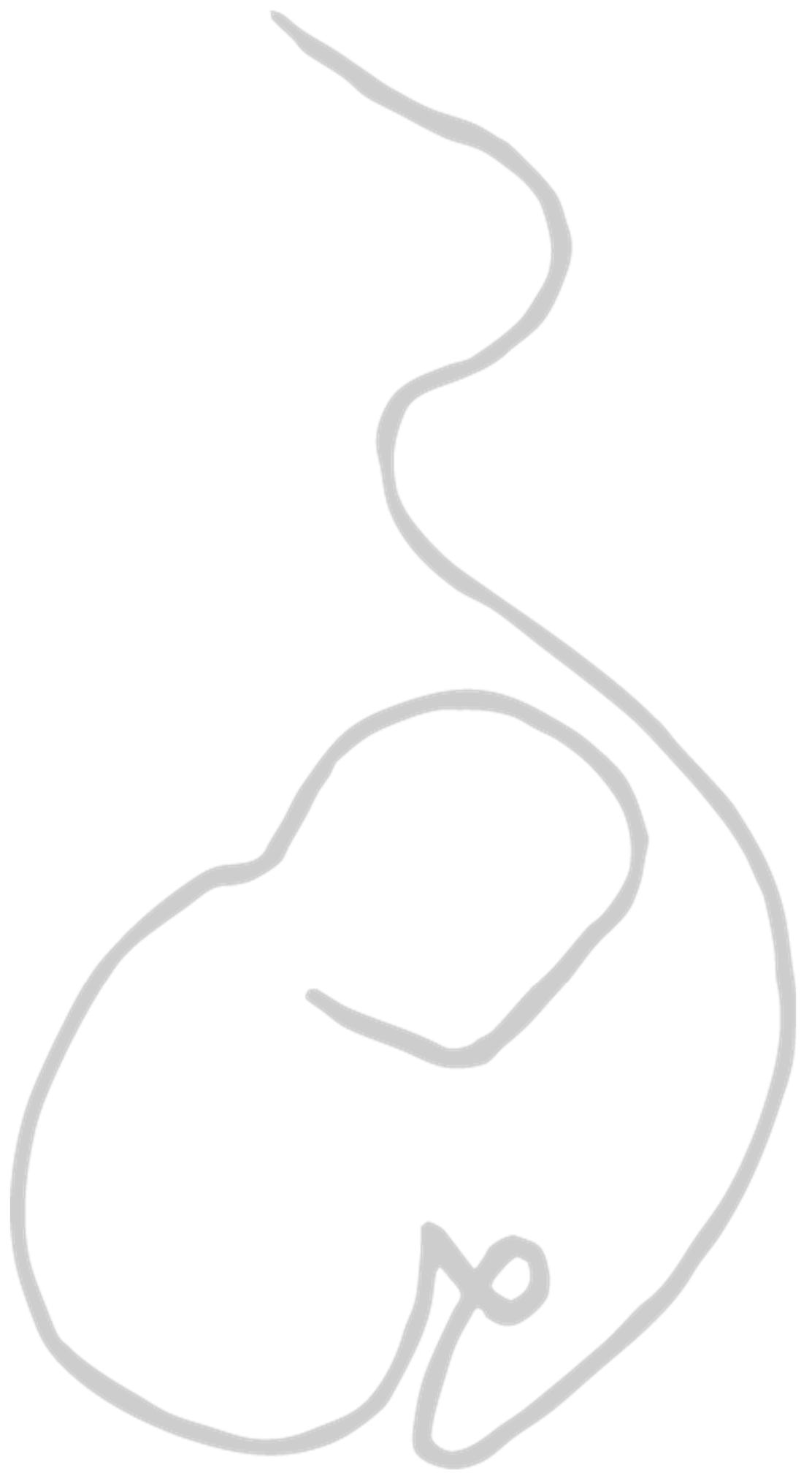

154 
Impact paragraph 


\section{Objective and findings}

The prevalence of obesity has nearly doubled since 1980 [1], resulting in $52 \%$ of the European Union's (EU's) adult population being overweight and $16 \%$ being obese [2]. At the same time, the prevalence of asthma has increased over the past few decades [3], leading to a current asthma prevalence of $5.4 \%$ in the EU [4]. Furthermore, respiratory infections are currently the fourth leading cause of both death and burden of disease in the world [5]. Next to their detrimental effects on a patient's health status and quality of life, these conditions have one thing in common: their development is associated with the fat content or fatty acid composition of the maternal diet during pregnancy. Given the increasing prevalences of these metabolic, immunologic and pulmonary conditions, and considering the substantial health care costs that are associated with treating them, society may benefit from preventing these diseases by improving maternal nutrition during pregnancy.

Although the long-term phenotypic effects in offspring of the maternal dietary fat content or fatty acid composition have been studied extensively, the molecular responses and mechanisms that are involved in these effects remain largely unknown. Therefore, this thesis aimed to study the effects of maternal dietary fatty acids and high-fat diet on the molecular responses related to offspring immunologic, metabolic and pulmonary health. In chapter 2 we showed that a perinatal high-fat diet induced oxidative stress, and altered DNA methylation and gene expression of pathways involved in lipid metabolism, oxidative stress response and cell proliferation in offspring liver. In chapter 3, a maternal high-fat diet exacerbated ozone-induced effects on pulmonary oxidant status and the molecular control of mitophagy in female offspring. In chapter 4, a maternal fish oil diet, which contained high amounts of $n-3$ polyunsaturated fatty acids (PUFAs), not only affected ex vivo polarization of offspring $\mathrm{CD}^{+} \mathrm{T}$ cells, but also induced anemia in both dams and offspring, and hepatic steatosis in offspring. In chapter 5, we used a multivariate statistical approach to demonstrate that maternal fatty acid status was associated with offspring inflammatory markers, and that maternal Mead acid levels correlated with the risk of developing lung conditions.

\section{Scientific relevance}

The findings from this thesis may contribute to scientific advancement in a number of ways. For chapters 2 till 4, this contribution mainly consists of the addition of fundamental knowledge about the effects of maternal nutrition on offspring health. The effects of a perinatal high-fat diet in chapter 2 on offspring hepatic DNA methylation and gene expression were related to the known adverse effects on offspring metabolism induced by a maternal high-fat diet. These findings may suggest that the effects of maternal diet on 
offspring health are transmitted via epigenetics and the regulation of gene expression. Similarly, the effects of a perinatal high-fat diet in chapter $\mathbf{2}$ on oxidative stress in offspring liver and in chapter 3 on ozone-induced oxidative stress in offspring lung suggest that oxidative stress may play an important role in the effects of maternal high-fat diet on offspring health. The effect of a maternal fish oil diet in chapter 4 on ex vivo polarization of offspring $\mathrm{CD}^{+} \mathrm{T}$ cells may be involved in the anti-asthmatic effects of prenatal supplementation with n-3 PUFAs, given the importance of $C D 4^{+} T$ cell polarization in atopy development. Altogether, these findings contribute to a better understanding of the molecular responses that underlie the long-term phenotypic effects of maternal nutrition. Furthermore, these findings provide new leads for future studies to further unravel the mechanisms by which maternal exposures affect offspring health.

Chapter 5 mainly contributes to scientific advancement by demonstrating the use of a nonstandard statistical approach for the examination of associations between prenatal nutrition and offspring health. In this chapter, we applied the statistical model canonical correlation analysis to study the multivariate relation between maternal fatty acid status and child inflammation. Applying this multivariate approach in future studies may aid in discovering new insights in the relation between maternal diet and offspring health.

\section{Societal relevance}

Results from this thesis, together with findings from previous and future studies, may contribute to the establishment of new dietary advises that will aid to improve the health of children that are yet to be born. By improving the immunologic, metabolic and pulmonary health, these children will have a better quality of life. Next to this, health care costs may be substantially reduced. For instance, 500 million euros are spent each year in the Netherlands to treat the consequences of obesity and indirect costs are even 2 billion euros each year [6]. For asthma, direct healthcare costs are estimated to be 287 million euros per year, with costs related to sick leave adding another 650 million euros per year [7].

\section{Target groups}

On the short term, findings from this thesis are most relevant to policy makers. (Semi-) governmental institutions such as the Research Council (Gezondheidsraad) and the Nutrition Center (Voedingscentrum) will have to collect and critically review results from our studies, as well as from related previous and future studies, and translate them into specific dietary advices. These dietary advices will then be used by healthcare professionals such as midwives, dietitians, general practitioners and gynecologists to inform and advise women who are pregnant, or plan to get pregnant, and their partners about good nutrition 
before and during pregnancy. Furthermore, nutrition companies may develop food products and supplements to help women meet the requirements for good perinatal nutrition. In the end, all of this will help to improve the health, and therefore quality of life, of future generations. Society as a whole may benefit from this, through the reduction of health care costs.

\section{Communication of results}

The findings presented in this thesis have been shared with other researchers in the field through poster presentations and oral presentations at national and international conferences. Furthermore, results have been or will be published in peer-reviewed scientific journals: chapters 2 and 3 have been published, chapter 4 is under review and chapter 5 is in preparation for submission. All chapters have been or will be published under a Creative Commons license, meaning that they are openly and freely accessible to everyone. For communication to the general public, organizations like the Nutrition Center (Voedingscentrum) will have to translate findings from this thesis and from related publications into specific and understandable dietary advises. 


\section{References}

1. World Health Organization. Global status report on noncommunicable diseases 2014; World Health Organization: 2014.

2. Eurostat Data Browser. Available online: https://ec.europa.eu/eurostat/databrowser/view/hlth_ehis_bm1e/default/table?lang=en (accessed on 03 May 2021).

3. Eder, W.; Ege, M.J.; von Mutius, E. The asthma epidemic. New England Journal of Medicine 2006, 355, 2226-2235.

4. Eurostat Data Browser, Persons reporting a chronic disease. Available online: http://appsso.eurostat.ec.europa.eu/nui/show.do?dataset=hlth_ehis_cd1e\&lang=en (accessed on 23-06-2021).

5. World Health Organization. Global Health Estimates: Life expectancy and leading causes of death and disability. Available online: https://www.who.int/data/gho/data/themes/mortality-and-global-health-estimates (accessed on 13-08-2021).

6. Rijksinstituut voor Volksgezondheid en Milieu. Wat bereikt u met de inzet op overgewicht? Available online: https://www.loketgezondleven.nl/gezondheidsthema/overgewicht/watbereikt-u (accessed on 20-10-2021).

7. Rijksinstituut voor Volksgezondheid en Milieu. Maatschappelijke kosten voor astha, COPD en respiratoire allergie. Available online: https://www.rivm.nl/publicaties/maatschappelijke-kosten-voor-astma-copd-enrespiratoire-allergie (accessed on 20-10-2021). 


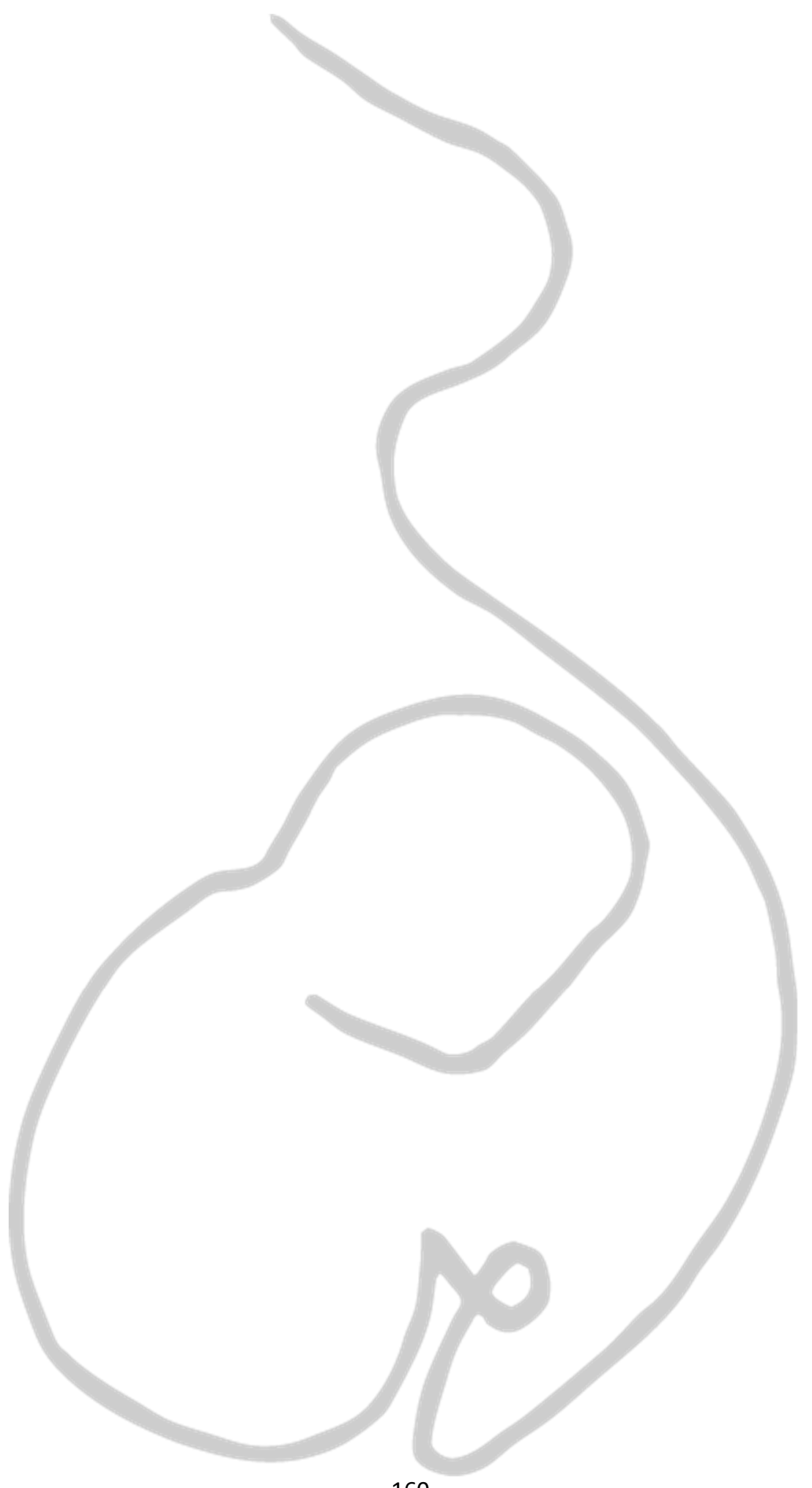


Samenvatting 


\section{Samenvatting}

Het vetgehalte en de vetzuursamenstelling van het maternale dieet tijdens de zwangerschap en lactatie beïnvloeden de ontwikkeling van het immuunsysteem, het metabolisme en de longen van nakomelingen, zelfs tot aan volwassen leeftijd. De moleculaire mechanismes die ten grondslag liggen aan deze relatie zijn echter grotendeels onbekend. Daarom was het doel van dit proefschrift om de effecten te bestuderen van maternale vetzuren of een hoog-vetdieet op de moleculaire reacties die betrokken zijn bij de immunologische, metabole en pulmonaire gezondheid van nakomelingen.

Perinatale blootstelling aan een hoog-vetdieet leidt tot een verslechterd metabolisme in nakomelingen. Men weet echter niet goed door welke mechanismes deze langetermijneffecten worden overgebracht. We hebben daarom in muizen het effect bestudeerd van een gecombineerd paternaal en maternaal hoog-vetdieet op genexpressie en DNA methylatie in de lever van volwassen nakomelingen. In hoofdstuk $\mathbf{2}$ hebben we aangetoond dat dit perinatale hoog-vetdieet veranderingen aanbracht in de expressie van genen die betrokken zijn bij vetmetabolisme, oxidatieve stress en celgroei. Verder veroorzaakte het perinatale dieet oxidatieve stress in de levers van nakomelingen. Daarnaast verhoogde het perinatale hoog-vetdieet de hoeveelheid DNA methylatie in bindingsplaatsen van transcriptiefactoren in het metabole gen Lpin1. We stellen de hypothese dat de veranderingen die we hebben waargenomen in deze studie ten grondslag liggen aan de bekende metabole verstoringen in nakomelingen ten gevolge van prenatale blootstelling aan een hoog-vetdieet.

Naast het beïnvloeden van metabolisme, kan een perinataal hoog-vetdieet ook nadelige effecten van luchtverontreiniging zoals ozon op longfunctie, -schade en -inflammatie verder verslechteren. $\mathrm{Er}$ wordt gesuggereerd dat deze verslechtering gerelateerd is aan disfunctioneren van mitochondriën. Het doel van hoofdstuk $\mathbf{3}$ was daarom om in ratten te onderzoeken of een dikmakend perinataal hoog-vetdieet veranderingen kan aanbrengen in de effecten die ozon heeft op mitochondriën en de oxidantstatus van longcellen. In deze studie verhoogde acute ozonblootstelling de oxidantstatus en de moleculaire regulatie van mitofagie in de longen van nakomelingen. In vrouwelijke nakomelingen verslechterde blootstelling aan een perinataal hoog-vetdieet deze effecten, terwijl in mannelijke nakomelingen de ozoneffecten niet afhankelijk waren van het maternale dieet. Deze bevindingen suggereren dat een perinataal hoog-vetdieet invloed heeft op ozongeïnduceerde effecten in oxidantstatus en de moleculaire regulatie van mitofagie in longen van vrouwelijke nakomelingen. 
Maternale suppletie met visvetzuren tijdens de zwangerschap is geassocieerd met een vermindering van astmaklachten. Ook hiervan zijn de onderliggende mechanismes onduidelijk. Aangezien polarisatie van $\mathrm{CD} 4^{+} \mathrm{T}$-lymfocyten een rol speelt in de ontwikkeling van astma, hebben we in hoofdstuk 4 de effecten bestudeerd van een perinataal visvetzurendieet op de ex vivo polarisatie van deze cellen in muisnakomelingen. In deze studie laten we zien dat het perinatale visvetzurendieet de extracellulaire hoeveelheden van cytokines vermindert, zowel tijdens Th1- als tijdens Th2-polarisatie. Daarnaast ontdekten we dat het perinatale visvetzurendieet niet alleen anemie (bloedarmoede) veroorzaakte in zowel de moeder als de nakomeling, maar ook leververvetting induceerde in nakomelingen. Deze resultaten tonen aan dat perinatale blootstelling aan hoge hoeveelheden visvetzuren zowel positieve als negatieve kan hebben in nakomelingen. Voor toekomstige studies die de perinatale effecten van visvetzuren willen bestuderen, is het dus belangrijk dat de doseringen nauwkeurig overwogen worden.

Het is aangetoond dat de ontwikkeling van inflammatoire longaandoeningen gerelateerd is aan het maternale dieet tijdens de zwangerschap. In eerdere studies waren bepalingen van de maternale vetzuurstatus en statistische analyses echter vaak suboptimaal. Daarom hebben we in hoofdstuk $\mathbf{5}$ de relatie tussen maternale vetzuurstatus en inflammatie in het kind op zevenjarige leeftijd bestudeerd door data van het MEFAB cohort te analyseren met behulp van canonical correlation analysis (CCA), een multivariaat statistisch model. In deze studie laten we zien dat maternale vetzuurstatus geassocieerd is met inflammatie in het kind op zevenjarige leeftijd en dat het vetzuur Meadzuur (C20:3n-9) een belangrijke bijdrage had in deze associatie. Verder waren maternale Meadzuur-plasmaspiegels op de dag van geboorte significant gecorreleerd met de kans op het ontwikkelen van inflammatoire longaandoeningen in het kind. Toekomstige studies moeten uitwijzen of het belang van Meadzuur is toe te wijzen aan het feit dat Meadzuur een biomarker is voor een tekort aan essentiële vetzuren, of aan de eigen pro-inflammatoire functies van Meadzuur, of mogelijk aan een combinatie van beiden.

Samengevat had dit proefschrift als doel om de effecten van maternale vetzuren en maternaal hoog-vetdieet te bestuderen op de moleculaire mechanismes gerelateerd aan de immunologische, metabole en pulmonaire gezondheid van de nakomeling. We hebben aangetoond dat een maternaal hoog-vetdieet in staat is genexpressie en DNA methylatie in de lever van nakomelingen aan te passen en daarnaast ook ozoneffecten op de long kan beïnvloeden in vrouwelijke nakomelingen. Verder hebben we aangetoond dat een maternaal visvetzurendieet van de ene kant een positief effect heeft op de ex vivo polarisatie van $\mathrm{CD}^{+} \mathrm{T}$ cellen, maar daarnaast ook negatieve effecten heeft op de gezondheid van zowel moeder als nakomelingen. Als laatste hebben we getoond dat maternale vetzuurstatus tijdens de zwangerschap geassocieerd is met inflammatie en de 
ontwikkeling van inflammatoire longaandoeningen in nakomelingen op latere leeftijd. Deze bevinden dragen bij aan meer kennis over hoe maternale voeding de gezondheid van nakomelingen beïnvloedt en kunnen mogelijk helpen om de gezondheid van toekomstige generaties te verbeteren. 


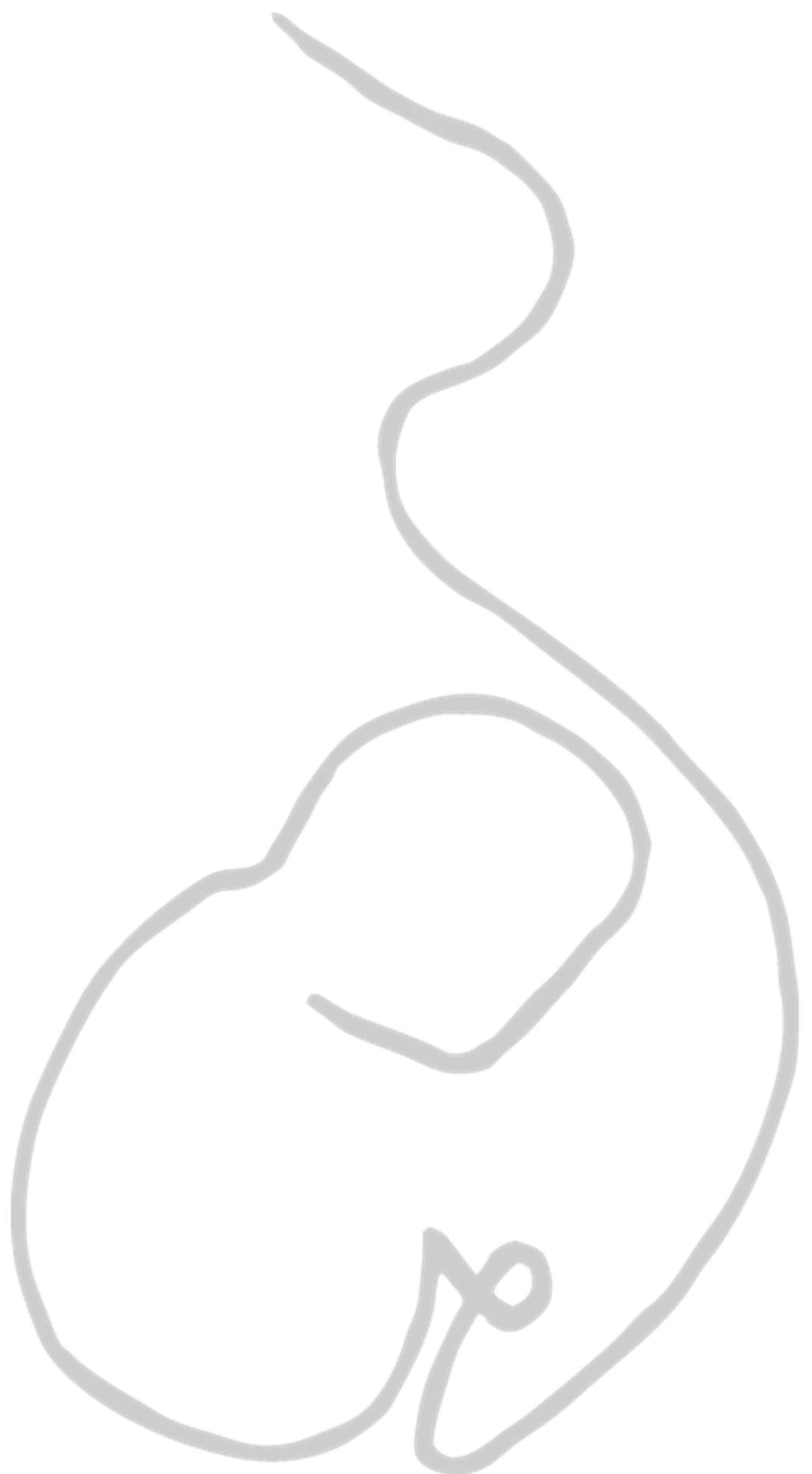


About the author 


\section{About the author}

Sven Rouschop was born on June $23^{\text {rd }} 1992$ in Maastricht, The Netherlands. He went to secondary school at Trevianum Scholengroep in Sittard, where he finished the Gymnasium in 2010. For his scientific education, Sven studied Molecular Life Sciences at Wageningen University. During the Bachelor's program, he did a minor in Health and Medical Biology and performed his internship at the department of Virology, where he studied cap-snatching of Schmallenberg virus in mosquito and mammalian cells. During the Master's program, he further specialized in Biomedical Research

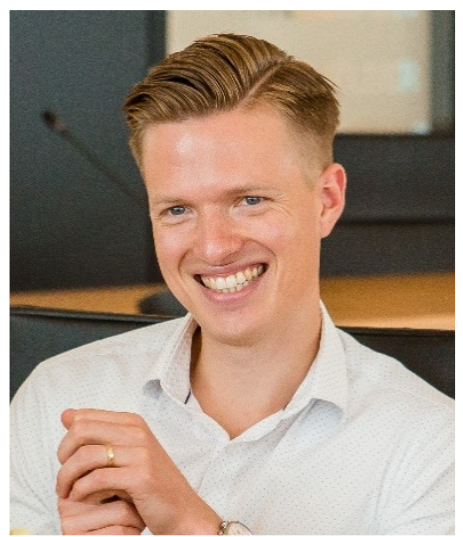
and performed two internships. For the first internship, Sven studied the effects of tastants on gut hormone secretion and their underlying signaling pathways at the Department of Nutrition and Pharmacology. The second internship was performed at MSD Animal Health, where he studied expression and purification of recombinant proteins for vaccine production. After graduating from university, Sven started his PhD research in 2016 at the department of Pharmacology and Toxicology at Maastricht University. During this PhD project, he studied the molecular effects of maternal dietary fat content and fatty acids on the immunologic, metabolic and pulmonary health in offspring. In 2017, Sven was awarded the KNAW Ter Meulen Beurs, which allowed him to visit the Risch lab at Salzburg University. In addition to performing research, Sven followed several courses related to statistics, animals research and clinical research, and visited (inter)national conferences, where he gave oral presentations and won two poster prizes. Furthermore, he was involved in teaching, for which he coordinated and supervised practical courses, supervised interns and guided problem-based learning groups. After finishing his PhD, Sven will continue his career at Hezelburcht Grant Consultancy. 


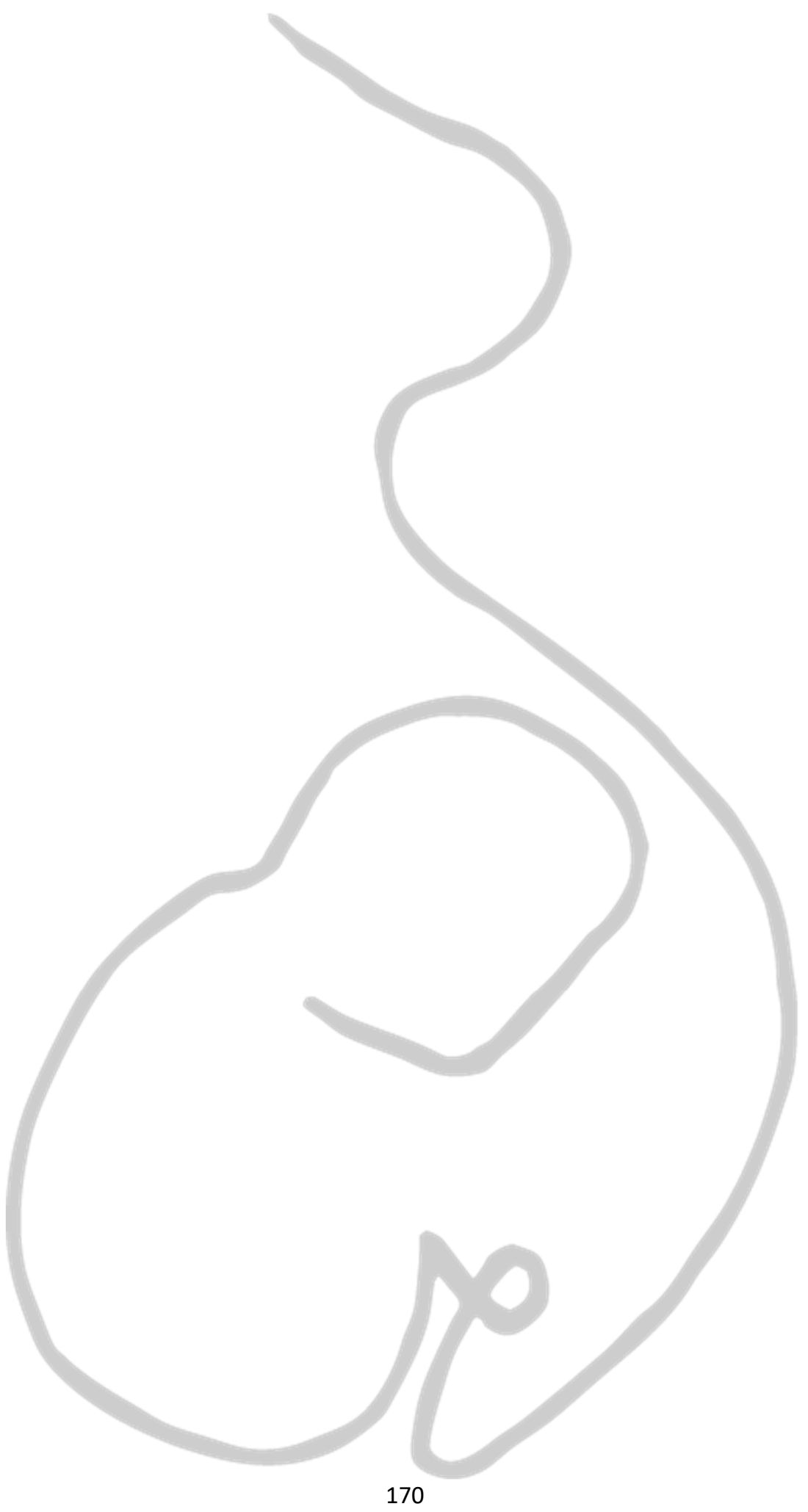


List of publications 


\section{List of publications}

Rouschop, S.H., Karl, T., Risch, A., van Ewijk, P.A., Schrauwen-Hinderling, V.B., Opperhuizen, A., van Schooten, F.J., Godschalk, R.W. (2019). Gene expression and DNA methylation as mechanisms of disturbed metabolism in offspring after exposure to a prenatal HF diet. Journal of lipid research, 60(7), 1250-1259.

Rouschop, S.H., Snow, S.J., Kodavanti, U.P., Drittij, M.J., Maas, L.M., Opperhuizen, A., van Schooten, F.J., Remels, A.H., Godschalk, R.W. (2021). Perinatal High-Fat Diet Influences Ozone-Induced Responses on Pulmonary Oxidant Status and the Molecular Control of Mitophagy in Female Rat Offspring. International journal of molecular sciences, 22(14), 7551.

Rouschop, S.H., Evers, L., Maas, L., Gijbels, M., Jolani, S., Germeraad, W.T.V., Meesters, D.M., Shiri-Sverdlov, R., van Schooten, F.J., Opperhuizen, A., Godschalk, R.W. Perinatal fish oil diet reduces ex vivo cytokine levels of polarizing $\mathrm{CD}^{+}{ }^{+} \mathrm{T}$ cells and induces anemia with microvesicular steatosis in mouse offspring. In preparation for submission.

Rouschop, S.H., Smolinska, A., Gielen, M., de Groot, R., Zeegers, M., Opperhuizen, A., van Schooten, F.J., Godschalk, R.W. Maternal fatty acid status during pregnancy versus offspring inflammatory markers: a canonical correlation analysis of the MEFAB cohort. In preparation for submission.

Stratakis, N., Gielen, M., Margetaki, K., Godschalk, R.W., Van der Wurff, I., Rouschop, S., Ibrahim, A., Antoniou, E., Chatzi, L., de Groot, R.G.M., Zeegers, M.P. (2017). Polyunsaturated fatty acid levels at birth and child-to-adult growth: Results from the MEFAB cohort. Prostaglandins, Leukotrienes and Essential Fatty Acids, 126, 72-78. 


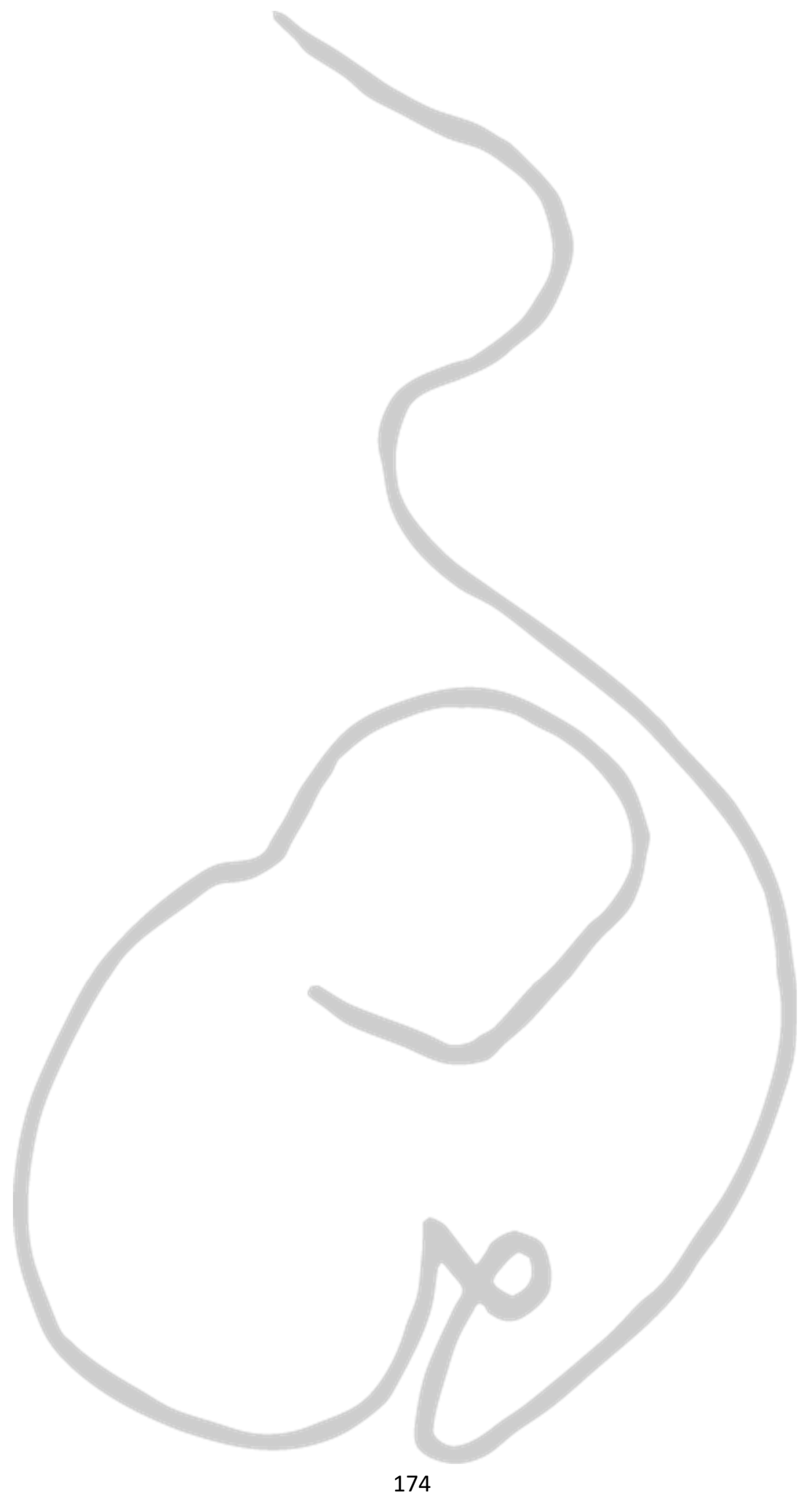




\section{Dankwoord}




\section{Dankwoord}

Dat was het dan, na meer dan vijf jaar is mijn proefschrift eindelijk af. Ik heb altijd met veel plezier aan mijn onderzoek gewerkt, maar nu het eenmaal zo ver is, ben ik opgelucht dat het klaar is. Op de kaft van dit proefschrift staat alleen mijn naam. Maar dat betekent niet dat ik het alleen gedaan heb. De afgelopen jaren heb ik hulp en steun gehad van een groot aantal mensen en deze wil ik hier een laatste keer bedanken.

Als eerste mijn promotieteam: Roger, Frederik-Jan en Antoon. Tijdens mijn studie was er maar één ding dat ik daarna wilde gaan doen: promoveren. Bedankt dat jullie me die kans hebben gegeven. Ik kreeg van jullie de ruimte om er mijn eigen project van te maken en daar heb ik veel van geleerd. Niet alleen hadden jullie altijd tijd voor overleg en feedback, maar ik keek ook uit naar onze meetings vanwege onze persoonlijke klik. Bedankt voor alles!

I would like to thank the assessment committee, prof. dr. Sijm, prof. dr. Calder, prof. dr. Nawrot, prof. dr. Blaak and dr. Al-Nasiry for the time and effort they spent on reviewing my thesis and for discussing my research with me at the defense.

Dan zijn er nog vele anderen die hebben bijgedragen aan de inhoud van dit proefschrift. Allereerst Lou en Marie-José. Jullie hebben me enorm geholpen met al het werk dat jullie voor mij gedaan hebben in het lab. Super bedankt voor deze ondersteuning en de prettige samenwerking.

Richard, Rik, Nicole (CPV), Marion (PATH/GEN), Wilfred (IMMUNO), Shahab (STAT), Dennis and Ronit (GEN), I have worked long and hard to make my own animal experiment succeed and I could not have done this without your help. Thank you for your assistance and for taking the time to answer all of my questions.

Angela, Tanja, Florian, Esther, Anna and Sissy of the Risch lab at Salzburg University, I have learned a lot from you and I had a wonderful experience when I visited your lab. Thank you for making me feel at home in Austria.

Alex, jouw contacten bij de EPA en onze daaropvolgende samenwerking hebben geleid tot een publicatie en een hoofdstuk in dit proefschrift. Bedankt voor je input en natuurlijk voor je nimmer-aflatende aanwezigheid bij sociale activiteiten. Urmila and Sam of the U.S. EPA, thank you for trusting me with your precious samples and our pleasant collaboration.

Agi, thank you for introducing me to the world of data analysis and for your help with the MEFAB study. Marij, Nikos, Maurice, Renate en Inge, bedankt voor de prettige samenwerking binnen het MEFAB team.

Secretaresses Marie-Claire en Josephina, dankzij jullie niet-aflatende hulp werden vergaderingen gepland, hotels geboekt, diners georganiseerd, facturen op tijd betaald, declaraties verwerkt en ga zo maar door. Bedankt voor deze ondersteuning. 
Laura, Skevi en overige stagiaires, ik vond het leuk om jullie te begeleiden en heb met plezier met jullie samengewerkt. Ik hoop dat ik jullie dingen geleerd heb waar jullie de rest van jullie carrière nog iets aan zullen hebben.

Naast de serieuze werkzaamheden die horen bij een promotietraject was er gelukkig ook altijd ruimte voor ontspanning en gezelligheid binnen de vakgroep.

Allereerst wil ik mijn kamergenoten Quan en Robert bedanken. Quan, you helped me find my way within the department and cheered me up with your never-ending happiness. Robert, ik geloof niet dat ik van iemand meer aanmoedigingen heb gehad tijdens mijn PhD dan van jou. Bedankt voor je positieve energie en je mentale support als paranimf. Philippe, ook jij bedankt dat je er straks tijdens de verdediging bij zult zijn om me bij te staan als paranimf. Dankjewel voor je hulp, alle koffiemomentjes en de gastvrijheid bij jouw thuis. Een van de fijne dingen aan het doen van een $\mathrm{PhD}$ is dat er altijd collega's zijn die in hetzelfde schuitje zitten. Kim, bedankt voor je hulp bij de dierstudie, de fietsritjes en de barbecues. Christy, Shan, Wenbo, George, Stefan, Narek, Sara, Timme, Charlotte, Misha, Mireille, Carmen, Rianne, Ming en Gesiele, bedankt voor alle gezelligheid tijdens de koffie, lunch, borrels, kerstdiners, gala's en barbecues.

Ook aan alle overige analisten en stafleden van PharTox, bedankt voor de ondersteuning, kritische vragen en gezelligheid.

Will en Alex van de spoelkeuken, bedank veur de auw kloete.

En als laatste wil ik de mensen bedanken die buiten het werk belangrijk waren.

Lieve familie: mama, Roger, papa, Hilde, Jordi, Jules, Famke, Aimée, Daphne, Anouk, Lauren, Paul, Marjet, Ed, Elien; bedankt voor jullie luisterend oor, jullie steun en liefde, jullie oprechte interesse en voor alle leuke en gezellige momenten die voor afleiding zorgden.

Buizerds, bedankt voor alle vakanties, stapavonden, verjaardagen en andere feestjes. Fanfies, bedankt voor de gezellige repetities, naborrels en fietstochten.

Tom, bedankt voor alle weekenden in Wageningen, Amsterdam en Maastricht, de vele speciaalbiertjes en sportieve activiteiten.

En dan echt als allerlaatste wil ik mijn lieve vrouw Merel bedanken. Bijna zes jaar geleden begonnen we samen aan ons promotietraject en in die tijd hebben we veel meegemaakt. We verhuisden naar Maastricht, zijn getrouwd en nu verwachten we ons eerste kindje. Werkinhoudelijk hebben we veel aan elkaar gehad. We konden samen klagen over afgewezen aanvragen, treuren over mislukte experimenten, maar ook vieren na behaalde publicaties en sparren over nieuwe ideeën. Maar bovenal zorgde je voor ontspanning en afleiding wanneer we gingen uiteten, naar een dans- of muziekvoorstelling, of gewoon lekker koken en een avondje bankhangen. Bedankt voor alles. 
Dankwoord 